\begin{abstract}
Origin, mechanics and properties of the Solar System are analyzed in the framework of Complete Relativity.

The analysis confirms the postulates and hypotheses of the theory with a high degree of confidence.

During the analysis, some new hypotheses have emerged. These are discussed and confirmed with various degrees of confidence. To increase confidence or refute some hypotheses, experimental verification is necessary.

Main conclusions are:

- Solar System is a scaled Carbon isotope with a nucleus in a condensed (bosonic) state and components in various vertically excited states,

- Earth is a living being of extremely introverted intelligence,

- life is common everywhere, albeit extroverted complex forms are present on planetary surfaces only during planetary neurogenesis,

- anthropogenic climate change is only a part (trigger from one perspective) of bigger global changes on Earth and in the Solar System during planetary neurogenesis,

- major extinction events are relative extinctions, a regular part of transformation and transfer of life in the process of planetary neurogenesis.
\end{abstract}




\title{
The Solar System: Nature and mechanics
}

\author{
Mario Ljubičić (Amenoum) \\ 108. brigade ZNG 43, 35252 Sibinj, Croatia mljubicic99@gmail.com
}

October 28, 2021

\section{Introduction}

Here I hypothesize that the Solar System is a large scale ${ }^{10} \mathrm{C}$ atom (10-Carbon isotope) and provide evidence for the equivalence of large $\left(\mathrm{U}_{1}\right)$ scale systems with standard $\left(\mathrm{U}_{0}\right)$ scale systems through the analysis of the Solar System in the context of Complete Relativity[1] (CR).

Note that ${ }^{10} \mathrm{C}$ isotope is unstable on standard scale, with a half-life of 19.3 seconds. Its apparent stability on $\mathrm{U}_{1}$ scale must be either the result of time dilation [due to scale difference] or inversion of stability between adjacent scales (vertical energy levels).

In case of inversion, stable systems on one scale would be unstable on the other and vice versa.

I hypothesize that the structure of planetary systems is the result of inflation of gravitational maxima from standard scale atoms, likely in the events of annihilation at event horizons (gravitational maxima) of a particular scale.

I propose that, in this process, electro-magnetic component of the general force has been exchanged with the neutral gravitational component resulting in the dominance of gravity over electro-magnetic force at this scale.

However, I also hypothesize that such exchange is natural on standard scale the atoms are cycling between polarized and neutral states (although durations in particular states might be inverted between scales).

Note that due to instability of ${ }^{10} \mathrm{~B}$ (decay product of ${ }^{10} \mathrm{C}$ and ${ }^{10} \mathrm{Be}$ ) at $\mathrm{U}_{1}$ scale, the Solar System must also be cycling between ${ }^{10} \mathrm{C}$ and ${ }^{10} \mathrm{Be}$ $\left({ }^{10} \mathrm{~B}\right.$ being the intermediate state). 
Implications of scale invariance of physical laws [and CR in general] on nature are large and some of these are further discussed and analyzed, primarily the implications on definition and understanding of life.

\section{Constants}

Here are the commonly used constants in the article.

The values of planetary constants are taken from NASA Planetary Fact Sheet[2].

\begin{tabular}{|c|c|c|}
\hline Description & Constant & Value \\
\hline $\begin{array}{l}\text { Neptune mass on scale } 1 \\
\text { Neptune mass on scale } 0\end{array}$ & $\begin{array}{l}\mathrm{M}_{U_{1}} \\
\mathrm{M}_{U_{0}}\end{array}$ & $\begin{array}{l}1.02413 * 10^{26} \mathrm{~kg} \\
\left(\quad 9.10938356 * 10^{-31} \mathrm{~kg}\right. \\
/ \quad 510998.9461 \mathrm{eV}) *( \\
510998.9461 \mathrm{eV}-11.260288 \\
\mathrm{eV})=9.109182827 * 10^{-31} \\
\mathrm{~kg}\end{array}$ \\
\hline Neptune orbital velocity & $\mathrm{v}_{U_{1}}$ & $5430 \mathrm{~m} / \mathrm{s}$ \\
\hline Neptune spin velocity & $\mathrm{s}_{U_{1}}$ & $2660 \mathrm{~m} / \mathrm{s}$ \\
\hline Neptune radius on scale 1 & $\mathrm{R}_{U_{1}}$ & $24622000 \mathrm{~m}$ \\
\hline Neptune radius on scale 0 & $\mathrm{R}_{U_{0}}$ & 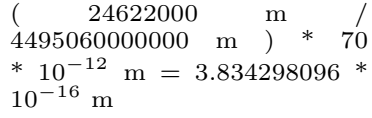 \\
\hline $\begin{array}{l}\text { Solar System charge radius }=\text { Neptune orbital ra- } \\
\text { dius }\end{array}$ & $\mathrm{r}_{U_{1}}$ & $4495060000000 \mathrm{~m}$ \\
\hline Sun mass & $\mathrm{M}_{\odot}$ & $1.988500 * 10^{30} \mathrm{~kg}$ \\
\hline Sun radius & $\mathrm{R}_{\odot}$ & $695735 \mathrm{~km}=695735000 \mathrm{~m}$ \\
\hline Earth mass & & $5.9723 * 10^{24} \mathrm{~kg}$ \\
\hline Carbon-12 atom mass & & $\begin{array}{l}1.992646547 * 10^{-23} \\
1.992646547 * 10^{-26} \mathrm{~kg}=\end{array}$ \\
\hline $\begin{array}{l}\text { Carbon-12 charge radius }=\text { Carbon-10 charge ra- } \\
\text { dius }\end{array}$ & $\mathrm{r}_{U_{0}}$ & $70 \mathrm{pm}=70 * 10^{-12} \mathrm{~m}$ \\
\hline Carbon-10 nucleus charge radius & & $2.708 * 10^{-15} \mathrm{~m}$ \\
\hline Carbon-10 nucleus mass & & $\begin{array}{l}10.016853 \mathrm{u}=1.663337576 \\
10^{-26} \mathrm{~kg}\end{array}$ \\
\hline Standard speed of light & $\mathrm{c}=\mathrm{c}_{0}$ & $2.99792458 * 10^{8} \mathrm{~m} / \mathrm{s}$ \\
\hline Standard electron mass & $\mathrm{M}_{e}$ & $9.10938356 * 10^{-31} \mathrm{~kg}$ \\
\hline
\end{tabular}

Table 1: Commonly used constants

\section{Definitions}

Here are the definitions of terms and expressions that may be used here. Note that these may be different than standard or common definitions in everyday use.

\subsection{Elementary charge}

Elementary particles relative to any system of certain scale may be generally charged. 
Physical interpretation (manifestation) of charge is dependable on environment, but each charge has a neutral component (vacuum), it's associated space, and electric component.

Electric component generally consists of 2 quanta of identical charge (dominant) and 1 quantum of opposite (anti) charge, which are strongly entangled.

There are no absolute monopoles. The concept of dominant charge and spatial superposition allow relative electric monopoles but relative magnetic monopoles require more complex structure as the magnetic field of an [relatively] elementary charge is the result of interaction and entanglement of at least two differently polarized electric charges and thus has two poles.

Spin momentum of charge quanta is quantized, by a relative constant $(\hbar)$ a quantum of momentum.

Suppose the value of each spin momentum is equal to $1 / 2 \hbar$ in value, and spins of two dominant charges are perpendicular to each other (having a [fixed] phase difference of $\pi / 2$ degrees). Two dominant charges now have a total magnetic spin momentum:

$$
S_{1}=\sqrt{\left(\frac{1}{2} \hbar\right)^{2}+\left(\frac{1}{2} \hbar\right)^{2}}=\frac{\sqrt{2}}{2} \hbar=\frac{1}{\sqrt{2}} \hbar
$$

a)

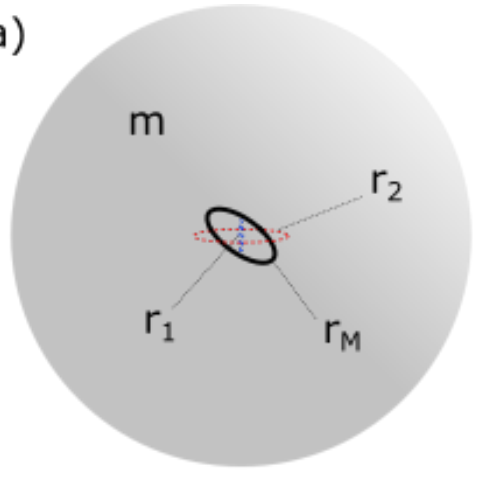

b)

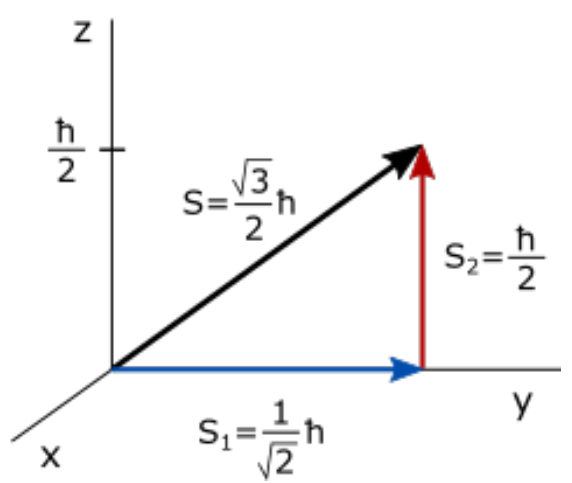

Figure 1: Spin momentum

Total spin momentum of the particle is thus:

$$
\vec{S}=\vec{S}_{1}+\vec{S}_{2}
$$

If the $\mathrm{S}_{2}$ (anti) charge momentum is perpendicular to $\mathrm{S}_{1}$, the value of total spin momentum is:

$$
S=\sqrt{\left(\frac{1}{\sqrt{2}} \hbar\right)^{2}+\left(\frac{1}{2} \hbar\right)^{2}}=\sqrt{\frac{1}{2}\left(\frac{1}{2}+1\right)} \hbar=\frac{\sqrt{3}}{2} \hbar
$$

Due to fixed $\pi / 2$ phase and equal value, influence of components of $S_{1}$ on the orientation [of the momentum projection] cancel, and the orientation of the 
projection of the momentum $\mathrm{S}$ on the axis of quantization will depend solely on the orientation of momentum $\mathrm{S}_{2}$.

With the applied magnetic field, projection of the momentum on the magnetic axis (ie. $\mathrm{z}$ ) will thus be oriented either up or down:

$$
S_{z}= \pm \frac{1}{2} \hbar
$$

Momentum $\mathrm{S}_{1}$ also has two possible orientations (left, right) and may represent chirality (handedness) of a particle.

Everything in a universe has dual nature, at least. A particle can not only be interpreted as a wave, but also as a living being (and vice versa).

Spin can thus be interpreted as sex (male/female - relating to a sexual reproduction organ), chirality as gender or sexuality (homo/hetero).

Fig. 1 a) shows charge in collapsed ground state (particle) with acquired real mass $\mathrm{m}$, charge radii $\mathrm{r}_{1}, \mathrm{r}_{2}$ and radius of imaginary mass $\mathrm{r}_{M}$.

It's momentum is quantized by $\hbar$, electric charge by e and gravitational force by $\hbar_{m g}$. The space of such particle is characterized by $\epsilon$ (electric permittivity) and $\mu$ (magnetic permeability).

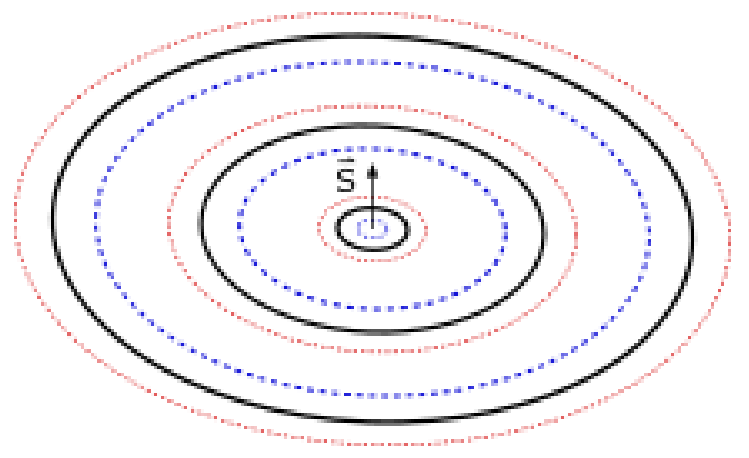

Figure 2: Charge wave

With decreasing environmental pressure (em/gravitational field interactions) a quantum may split into smaller quanta, spreading as far as possible, but still entangled, with a wave-like distribution of potential. Fig. 2 shows such unbound, free charge. Total momentum is the sum of individual momenta and equal to original momentum of the particle.

Fig. 3 a) shows strength of forces of a wave with distance from center (black $=$ gravitational force, blue and red $=$ electric force). Now each component (maximum) of a wave, starting from outer ones, can be excited independently, can change spin, merge with adjacent maxima and form moon charges.

This allows the charge to interact (interfere) with itself, exhibiting a wavelike nature.

Fig. $3 \mathrm{~b}$ ) shows how the space of the same particle can be modified by interaction with another particle - essentially, the electric force has been exchanged 
a)

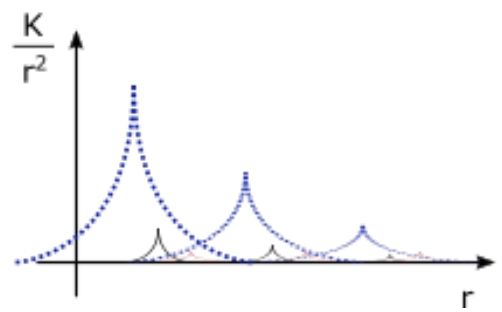

b)

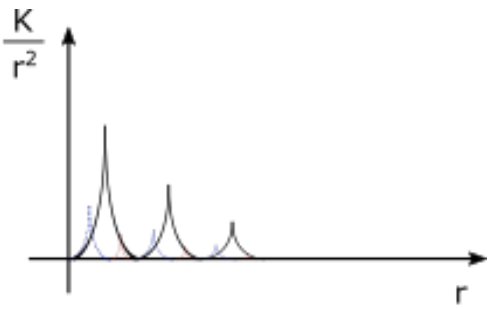

Figure 3: Charge wave forces

for gravitational force. Such interaction may also collapse the wave into a particle with moon charges, where the number of moons depends on the equilibrium point of interaction (difference in energy of interacting particles).

Note that it is possible for the effect to be strongly localized - local space may be modified to attenuate one force and strengthen the other, while particles outside that space may not feel such [degree of] change.

\subsubsection{Equilibrium and nature of forces}

Equilibrium state of 3 components of charge is maintained through rotation. Due to rotation of local space, general force is a centripetal force and in stable orbitals equal to centrifugal force.

In case of a completely neutral (gravitational) force:

$$
\frac{m v^{2}}{r}=\frac{G M m}{r^{2}}
$$

This is established when angular velocity of the orbiting body becomes equal to angular velocity of space (effective graviton, or gravitational field tube):

$$
v=v_{s}=\sqrt{\frac{G M}{r}}
$$

If the body increases velocity $\left(\mathrm{v}>\mathrm{v}_{s}\right)$, centrifugal force becomes greater than gravitational force and now acts as a fictitious repulsive force.

For $\mathrm{v}<\mathrm{v}_{s}$, gravitational force is higher than centrifugal force, and the body feels attractive force.

Nature (polarization) of the force can thus be changed with a change in radii (expansion/collapse) of gravitational maxima.

This allows for electro-magnetic force to be a fictitious force - a result of radii change of gravitational maxima due to absorption and emission of energy.

Note that electric polarization of atoms is done through emission and absorption of electrons, which is affecting the atom radius - positive polarization will generally decrease radius (in common atom radius in- 
terpretation), while negative will increase it.

However, when radius is proportional to gravity, positive polarization would create repulsion, while negative would create attraction. Thus, the sole change in radii cannot be the equivalent of electro-magnetic force, as nature of EM force (attraction/repulsion) depends on the pair of interacting charges, not solely on the polarity of individual charge.

Thus, electric polarization of a graviton will, as hypothesized in CR, require deformation, creation of a bipolar structure. Nature and strength of force thus become dependent on correlation (entanglement) between particles.

A neutral graviton effectively curves space, proportionally to its scale and isotropically in ideal (completely neutral) case. However, with electric polarization, isotropic large scale curvature decreases and becomes quantized (fragmented) into smaller scale curvatures concentrated in [magnetic field] lines (small scale tubes).

Such particle will not strongly gravitationally attract particles of the same scale, but it will strongly interact with particles in the same configuration (having magnetic field tubes of the same scale). If this interaction is widening the tubes (increasing energy) of entanglement, the particles will be attracted, otherwise repelled.

Even if orbital changes are not electro-magnetic in nature, such changes imply radial polarization of reference frames, thus a reference frame can be polarized even if its mass is purely gravitational, and this will be reflected in a relativistic $(\omega)$ factor.

However, there are no absolutely pure gravitational reference frames and changes in stable orbits may generally happen with the exchange of gravitational for electro-magnetic potential.

In that case, gravitational polarization becomes electric polarization.

\subsection{Primary atom radius}

Generally, radius of the atom is equal to the radius of its outermost electron orbit.

Primary radius of the atom is equal to the orbital radius of its outermost primary component.

At minimum, it is equal to the general (outermost electron orbit) radius of the atom. However, at equilibrium - with all primary neutrinos present, it may be over twice that radius.

\subsection{MAU}

MAU or Mars relative Astronomical Unit is a unit of distance, relative to the orbit of the outermost positive charge in an atom. $1 \mathrm{MAU}$ is equal to the 
distance of such charge from the nucleus.

On $\mathrm{U}_{1}$ scale ${ }^{10} \mathrm{C}$ atom, $1 \mathrm{MAU}$ is equal to the distance of Mars from the Sun.

\subsection{Weak nuclear decay}

Weak nuclear decay transforms a neutron into a proton or vice versa. If these are parts of an atom, this is nuclear transmutation - transformation of one atom of an element into an atom of another element.

With scale invariance of gravitational fields, neutrinos and anti-neutrinos can be, like electrons, bound to atomic nuclei (and, as other fermions, grouped into pairs). In equilibrium, the number of bound electron (e) neutrinos and electron anti-neutrinos within the [primary] radius of the atom correspond to the number of protons and neutrons, respectively. These are, together with nuclei and electrons, primary components of the atom.

Decay process involves annihilation of neutrinos and anti-neutrinos.

\subsection{1 $\beta^{-}$decay}

Transformation of a neutron to a proton, with the emission of excess energy:

$$
n \rightarrow p^{+}+\Delta E
$$

Here, bound non-primary e neutrino and bound primary e anti-neutrino annihilate to produce, depending on energy, either an electron/positron $\left(\mathrm{e}^{-} / \mathrm{e}^{+}\right)$pair, or up/anti-up quark pair:

$$
e_{v}+\bar{v}_{e} \rightarrow\left(e^{-}+e^{+}\right) \|\left(u^{+}+u^{-}\right)
$$

In case of electron/positron production, positron further partially annihilates with the down quark (here, both are composite particles), producing neutrino/antineutrino pair and up quark:

$$
e^{+}+d^{-} \rightarrow u^{+}+v_{e}+\bar{v}_{e}
$$

Neutrino bounds to the atom [as a primary component], while anti-neutrino and electron are ejected in a spin paired state (boson), before separating again:

$$
e^{-}+\bar{v}_{e} \rightarrow W^{-} \rightarrow e^{-}+\bar{v}_{e}
$$

In case of up/anti-up quark production in the first step, the up quark is absorbed, while anti-up quark pairs with the down quark before ejection:

$$
u^{-}+d^{-} \rightarrow W^{-} \rightarrow u^{-}+d^{-}
$$


Note that a decay of $\mathrm{W}^{-}$into an electron and anti-neutrino even when it is created from anti-up and down quarks would suggest that charge in electron is a composite of $1 / 3$ and $2 / 3$ charge quanta. In the decay of a proton to neutron through electron capture, electron could then [inverse] decay to $\mathrm{u}^{-}$and $\mathrm{d}^{-}$by pairing with an anti-neutrino (inflating to $\mathrm{W}^{-}$ boson), $\mathrm{u}^{-}$would annihilate with $\mathrm{u}^{+}$, leaving 2 down and 1 up quark, forming a neutron.

Outside of atom, the pairing is unstable (short-lived), except at extreme conditions.

Note that, in this case, to conserve equilibrium conditions, one of bound non-primary e neutrinos must reduce its orbit to become a primary component. $\beta^{-}$decay is the effective transformation of a down quark to up quark of the atom nucleus.

Note that a W boson has a rest mass over 80 times that of a neutron and orders of magnitude more than that of down and up quarks.

Thus, the production of a $\mathrm{W}$ boson is apparently a violation of energy conservation. In QM this is solved with time-energy uncertainty principle which allows production of such particles out of vacuum providing they decay quickly (lifetime of a $\mathrm{W}$ boson is $10^{-25}$ seconds).

However, mass of the boson is also considered variable with probability of deviation from rest mass decreasing fast with the amount of deviation, thus, making the probability of beta decay proportional to creation of a low mass $\mathrm{W}$ boson.

In reality, there is no violation of energy conservation and high mass of a $\mathrm{W}$ boson is actually the result of conservation of energy due to momentum - energy equivalence (note that, per CR postulates, even rest mass has a momentum), where one component of the angular momentum is exchanged for the other. In this case, the angular momentum of a particle orbiting the nucleus is collapsed [localized] to a spin momentum, where radius has been exchanged for mass.

This is, generally, the process of conversion of a polarized component of the general force into a neutral (gravitational) component - effectively, the exchange of charge for mass.

If this is temporary, like in case of $\beta$ decay, radius is inflated again (restoring em component) and two components of the force are again separated (concentrated) into multiple particles (although neither can be absolutely zero for any particle).

Thus, although $\mathrm{W}$ boson is charged, and charge is conserved between the initial and final state of the system, it is not conserved in the boson itself (unless created mass is indeed extremely low compared to rest mass) - 
otherwise the conservation of energy would be violated.

It will be shown later that, in reality, time-energy uncertainty indeed manifests itself in the exchange of angular momentum components.

\subsection{2 $\beta^{+}$decay}

Transformation of a proton to a neutron, with the emission of excess energy:

$$
p^{+} \rightarrow n+\Delta E
$$

Here, bound primary e neutrino and bound non-primary e anti-neutrino annihilate to produce either an electron/positron $\left(\mathrm{e}^{-} / \mathrm{e}^{+}\right)$pair, or down/anti-down quark pair:

$$
e_{v}+\bar{v}_{e} \rightarrow\left(e^{-}+e^{+}\right) \|\left(d^{+}+d^{-}\right)
$$

In case of electron/positron production, electron further partially annihilates with the up quark (here, both are composite particles), producing neutrino/antineutrino pair and a down quark:

$$
e^{-}+u^{+} \rightarrow d^{-}+v_{e}+\bar{v}_{e}
$$

The anti-neutrino bounds to the atom [as a primary component], while neutrino and positron are ejected in a spin paired state (boson), before separating again:

$$
e^{+}+v_{e} \rightarrow W^{+} \rightarrow e^{+}+v_{e}
$$

In case of down/anti-down quark production in the first step, the down quark is absorbed, while anti-down quark pairs with the up quark before ejection:

$$
u^{+}+d^{+} \rightarrow W^{+} \rightarrow u^{+}+d^{+}
$$

Note that, in this case, to conserve equilibrium conditions, one of bound nonprimary e anti-neutrinos must reduce its orbit to become a primary component. $\beta^{+}$decay is the effective transformation of an up quark to down quark of the atom nucleus.

\subsubsection{Inverse $\beta$ decay}

Transformation of a proton to a neutron by electron anti-neutrino scattering. Generally, this interaction will occur when the atom is not in equilibrium, more specifically - the number of bound e neutrinos is lower than the number of protons.

$$
\bar{v}_{e}+p^{+} \rightarrow e^{+}+n
$$


In this process, e anti-neutrino annihilates with a bound non-primary e neutrino, initiating a $\beta^{+}$decay with electron/positron product:

$$
\begin{gathered}
e_{v}+\bar{v}_{e} \rightarrow e^{-}+e^{+} \\
e^{-}+u^{+} \rightarrow d^{-}+v_{e}+\bar{v}_{e}
\end{gathered}
$$

However, since the number of bound primary e neutrinos was initially lower than the number of protons, now even the created neutrino is bound (as a non-primary component) rather than ejected with a positron:

$$
e^{+} \rightarrow e^{+}
$$

\subsubsection{Electron capture}

Transformation of a proton to a neutron by electron capture.

$$
p^{+}+e^{-} \rightarrow v_{e}+n
$$

Bound electrons induce the creation of positrons from the atom nucleus, filling its outer energy levels. In low energy conditions this may not be possible and one of the innermost electrons may be captured to fill the vacant level. However, the electron in this level is highly unstable, it is attracted to the outer proton core where it partially annihilates with the up quark, proceeding further as $\beta^{+}$ decay:

$$
e^{-}+u^{+} \rightarrow d^{-}+v_{e}+\bar{v}_{e}
$$

The anti-neutrino bounds to the atom as a primary component, while neutrino gets ejected. Like in case of inverse $\beta$ decay, there is no $\mathrm{W}$ boson creation as no positrons were created:

$$
v_{e} \rightarrow v_{e}
$$

\section{Initial structure hypothesis}

In planetary systems, outer (gas) planets are [groups of] electrons, while inner (terrestrial) planets are [groups of] positrons whose gravitational maxima have been extracted from the system nucleus to balance the electrons.

A planet can be in 1e or 2e configuration, while the star is a superposition of nuclei partons (quarks). Inner and outer dwarf planets in a planetary system are bound anti-neutrinos and neutrinos, respectively.

Primary components of the Solar System are shown on Fig. 4.

Note that components of momentum are exchangeable and it is the reason why bound neutrinos/anti-neutrinos have significantly inflated real mass compared to free neutrinos/anti-neutrinos. 


\section{0}

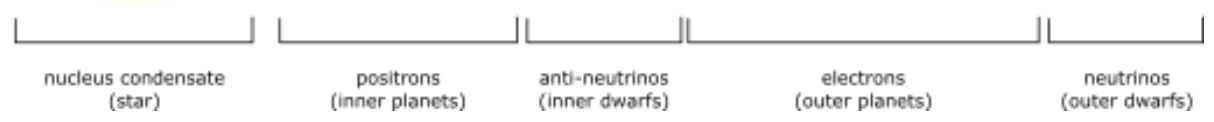

Figure 4: Primary components of the Solar System (planet images source: Pixabay/OpenClipart-Vectors ${ }^{3}$ )

a)

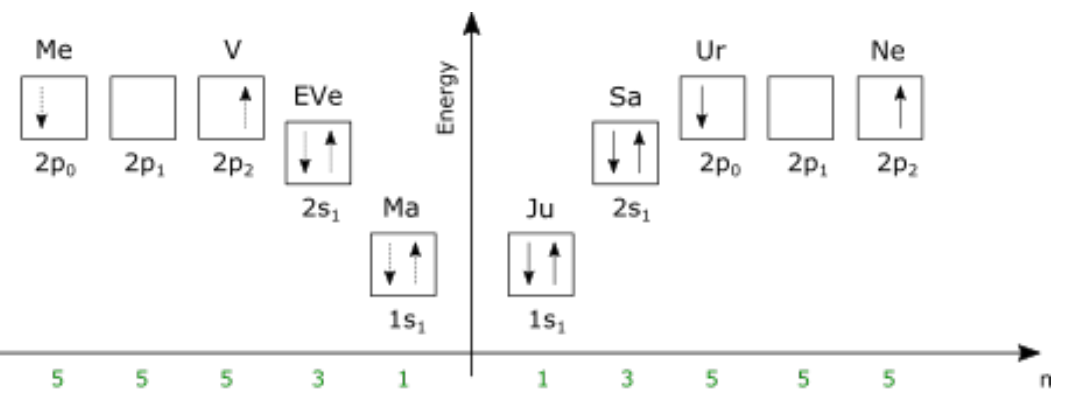

b)

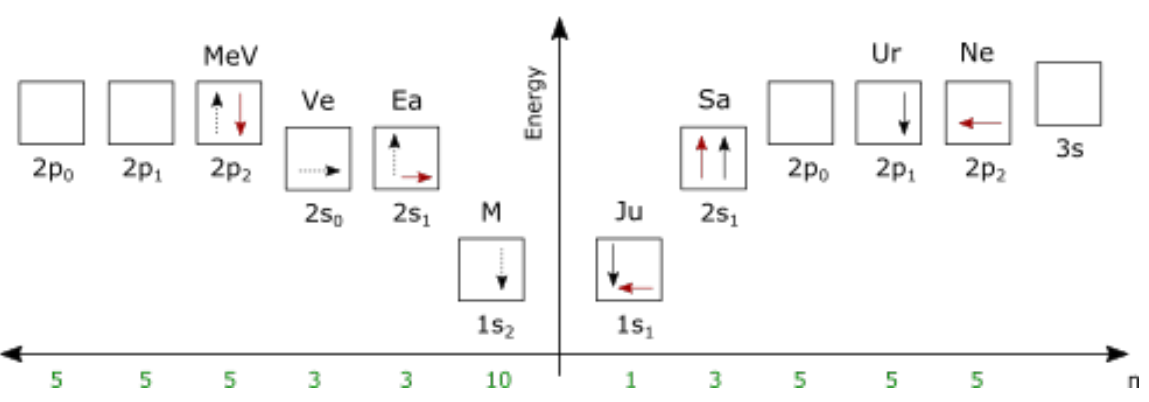

Figure 5: a) stable ${ }^{12} \mathrm{C}$ energy levels b) current Solar System $\left({ }^{10} \mathrm{C}\right)$ energy levels 
The current Solar System is in a ${ }^{10} \mathrm{C}$ atom configuration, in transition to ${ }^{10} \mathrm{Be}$ through $\beta^{+}$decay.

Fig. 5 a) shows the configuration of a ${ }^{12} \mathrm{C}$ atom (stable on standard scale, unstable on $U_{1}$ ), on the left is the configuration of positrons, on the right is the configuration of electrons.

Fig. $5 \mathrm{~b}$ ) shows the possible configuration of a ${ }^{10} \mathrm{C}$ atom (unstable on standard scale, relatively stable on $\mathrm{U}_{1}$ scale).

Note that splitting of $\mathrm{s}$ levels on the left side should be attributed to lack of neutrons, as they provide neutral gravitational energy to inner planets.

This generally does not happen on the right side where this energy is provided by protons.

Note also that, due to condensation (the system may be carbon-like, not carbon), principal quantum number has an imaginary value (n) and effective value $(\mathrm{N})$ which here is either 1 or 2 .

Note also that 2 particles are allowed per sub-shell and there is no reason for a lone electron not to pair up with a bound neutrino, forming a [W] boson, although such pairing may be extremely unstable at room temperature/density, oscillating in existence.

\subsection{General deduction of quantum structure}

Here is an example how the element and exact isotope species can be determined from the number and types of planets.

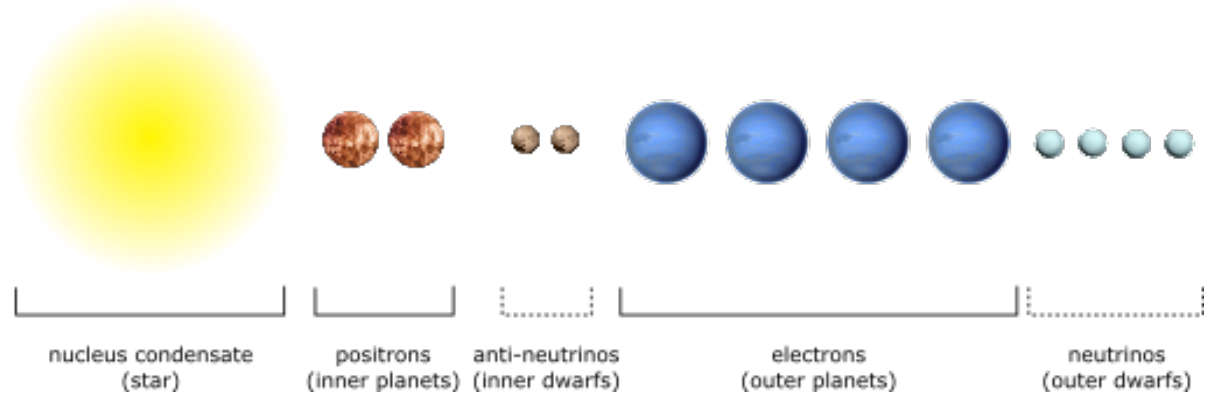

Figure 6: Primary components of the TOI-178 System (planet images source: Pixabay/OpenClipart-Vectors ${ }^{4}$ )

The discovered (star, planets) and hypothesized (dwarf planets) components of TOI-178 system are shown on Fig. 6 .

With the assumption of maximum 2 electrons (positrons) per planet, the TOI-178 system has these restrictions on the number of particles: 
- 2 terrestrial planets limit the number of positrons to $2-4$,

- 4 gas planets limit the number of electrons to 4 - 8 .

Since the intersection of the two groups contains only one solution (4), the TOI-178 system must be a Beryllium atom.

If the number of terrestrial planets corresponds to number of neutrons, this must be a ${ }^{6} \mathrm{Be}$ isotope.

This can be confirmed by comparing the mass of the TOI-178 system [star] with the mass of the Sun. Assuming that the Solar System is ${ }^{10} \mathrm{C}$ (or ${ }^{10} \mathrm{Be}$ ), the determined mass of TOI-178 $\left(0.647^{+0.035 /-0.032} \mathrm{M}_{\odot}[5]\right)$ agrees well with the hypothesis.

However, the measured mass is still somewhat larger than expected - reasons for this will be discussed later.

Note that it is also possible for the number of terrestrial planets to actually reduce with the increasing number of neutrons due to increased gravitational potential provided by neutrons, but this also requires either low [properly scaled] temperatures/densities for boson condensation of charges beyond the 2e configuration or excessive number of neutrons compared to protons.

Note also that, in heavy elements, due to condensation of mass and with no significant change in atomic radii, there is a possibility for all planets of a system to be gaseous giants. However, equivalents of dwarf planets should exist in between positively and negatively charged giants - in that case, these should be of significantly lower mass and may be equivalents of terrestrial planets with no significant magnetic dipoles.

The number of bound [primary] anti-neutrinos should also correspond to number of neutrons, while the number of bound [primary] neutrinos should correspond to the number of protons.

Note that, while bound anti-neutrinos/neutrinos should correspond to number of neutrons/protons, they will not necessarily be in the same configuration as positrons/electrons.

Thus, it is possible that TOI-178 has a single inner dwarf planet (holding 2 anti-neutrinos) instead of two, and two outer primary dwarf planets instead of four.

Additional particles may also be bound to the system, however, orbits of these should lie beyond the primary components, unless these are lower mass particles with no distinct gravitational maximum (asteroids, comets).

Note also that, with the exception of the innermost planet, planets of 
the TOI-178 are in orbital resonance (18:9:6:4:3). The pattern does suggest one additional particle (or a binary) between the terrestrial and gas planets, one that would complete 13 revolutions for every 18 revolutions of the second planet (pattern 18:13:9:6:4:3).

\section{Quantum nature}

Solar System appears to be a Carbon-10 atom in the current state. Due to extreme conditions some of its components are at the lowest energy level - multiple nucleons have condensed into a single nucleus, orbitals are two dimensional (collapsed from spherical cloud structure), highly aligned (same plane), and momentum carriers are (scaled) point like structures.

Scale invariance of physical laws requires that non-dimensional ratios - those of radii, masses and velocities (energies in general) in two systems of the same species (carbon in this case) but of different scale are equal.

Radius of the outermost electron of ${ }^{10} \mathrm{C}$ can be obtained from Neptune spin and orbital radius:

$$
\begin{aligned}
\frac{\text { Neptune spin radius }}{\text { Neptune orbital radius }} & =\frac{10 \mathrm{C} \text { outermost electron spin radius }}{10 \mathrm{C} \text { outermost electron orbital radius }} \\
& =\frac{R_{U_{1}}}{r_{U_{1}}}=\frac{R_{U_{0}}}{r_{U_{0}}}
\end{aligned}
$$

This gives electron radius $\mathrm{R}_{U 0}=3.834298096 * 10^{-16} \mathrm{~m}$. Note that radii of particles inside the atom can be different than outside of atom.

Generally, radii are affected by kinetic energy and oscillate with mass.

Sun core radius from ${ }^{10} \mathrm{C}$ nucleus radius and outermost electron radius:

$$
\frac{10 \mathrm{C} \text { nucleus charge radius }}{10 \mathrm{C} \text { outermost electron spin radius }}=\frac{\text { Sun core radius }}{\text { Neptune spin radius }}
$$

The above gives Sun core radius of $173894.6069 \mathrm{~km}$, or $1 / 4$ of the apparent Sun radius, in agreement with experimentally obtained values of Sun core size. More precisely, this is the Sun outer core [discontinuity] radius and also [approximately] $\mathrm{U}_{1}$ classical electron radius.

Proton radius approximation:

$$
\frac{\text { Sun radius }}{\text { Solar System charge radius }}=\frac{P}{N} \frac{10 * \text { proton radius }}{\text { Carbon-10 charge radius }}
$$

The factor $\mathrm{P} / \mathrm{N}=6 / 4=3 / 2$ is the ratio of protons to neutrons in Carbon- 10 atom, factor 10 is the number of nucleons $(\mathrm{P}+\mathrm{N})$.

The above gives $0.7222958833 * 10^{-15} \mathrm{~m}=0.7222958833 \mathrm{fm}$ for the proton radius, close to experimentally obtained value of $0.8414(19) \mathrm{fm}$ (2018 CODATA[6]). 
Same result can be obtained by using spin radii:

$$
\frac{\text { Sun radius }}{\text { Neptune spin radius }}=\frac{P}{N} \frac{10 * \text { proton radius }}{10 \mathrm{C} \text { outermost electron spin radius }}
$$

A precise value can be obtained by taking into account the influence of quarks instead of $\mathrm{P} / \mathrm{N}$ (this will be elaborated later):

$$
\frac{\text { Sun radius }}{\text { Solar System charge radius }}\left[\left(\frac{2}{3}\right)^{2}+\frac{1}{3}\right]=\frac{10 * \text { proton radius }}{\text { Carbon-10 charge radius }}
$$

which gives $0.8426785306 \mathrm{fm}$, a value in agreement with the CODATA value.

Radius of a proton is not constant, it is expected to be shrinking as the Solar System expands during weak evolution of the current state $(6 \mathrm{p} 4 \mathrm{n})$.

Comparing masses:

$$
\frac{\text { Sun mass }}{\text { Neptune mass }} \approx \frac{10 \mathrm{C} \text { nucleus mass }}{10 \mathrm{C} \text { outermost electron mass }}
$$

This gives:

$$
19416.48033 \approx 18260.0087
$$

The above shows mass ratios agree not only to the order of magnitude but are actually very close in value. The excess energy is:

$$
\begin{aligned}
\Delta M & =\text { Sun mass }-\frac{10 \mathrm{C} \text { nucleus mass }}{10 \mathrm{C} \text { outermost electron mass }} \text { Neptune mass } \\
& =1.18437729 * 10^{29} \mathrm{~kg} \approx 6 \% \text { Sun mass }
\end{aligned}
$$

and it must be the kinetic energy of the Solar System (discrepancy arises due to non-invariant reference frames in the mass measurement - the mass of a standard ${ }^{10} \mathrm{C}$ atom is measured from an external frame, while the mass of the Solar System is derived from within that system and improperly treated as rest mass).

Although the Solar System is at rest relative to us, kinetic energy of the system due to interaction with the intergalactic $\left(\mathrm{U}_{1}\right)$ medium must be stored somewhere in the system. This capacitor is the gravitational field (imaginary mass).

Note that this implies non-isotropic storage of kinetic energy as gravitational potential, such that it is mostly concentrated in the Sun - density distribution following the gravitational law $\left(1 / \mathrm{r}^{2}\right)$.

From this one can calculate the scaled speed of light for the $\mathrm{U}_{1}$ scale $\left(\mathrm{c}_{1}\right)$ :

$$
M=M_{\odot}-\Delta M=1.870062271 * 10^{30} \mathrm{~kg}
$$




$$
\begin{gathered}
v=v_{s}+v_{p} \\
M_{\odot}=\frac{M}{\sqrt{1-\frac{v^{2}}{c_{1}^{2}}}} \\
c_{1}=\frac{v}{\sqrt{1-\frac{M^{2}}{M_{\odot}^{2}}}}
\end{gathered}
$$

where $\mathrm{v}$ is the cumulative velocity against the CMB (Constant Microwave Background) radiation, a sum of secondary velocity $\mathrm{v}_{s}$ (velocity of the Solar System against $\mathrm{CMB}$ ) and primary velocity $\mathrm{v}_{p}$ (equal to velocity of the local galactic group against $\mathrm{CMB}$ ).

For $\mathrm{v}_{s}=368 \mathrm{~km} / \mathrm{s}$ and $\mathrm{v}_{p}=628 \mathrm{~km} / \mathrm{s}$ :

$$
c_{1}=2.93 * 10^{6} \mathrm{~m} / \mathrm{s}
$$

Obtained $c_{1}$ will be confirmed later in a different calculation.

At first, it may seem that this calculation cannot be valid since both velocities are relative to CMB and $\mathrm{v}_{p}$ should not be included in calculation. However, the obtained $c_{1}$ is confirmed later in other calculations. This puts certain constraints on Sun's evolution, implying that Sun's gravitational maximum was, after inflation, accelerated to $628+368 \mathrm{~km} / \mathrm{s}$ (additionally inflating) in the direction of local galactic group, then decelerated to $368 \mathrm{~km} / \mathrm{s}$, however, not loosing the acquired energy (it is yet to loose it).

This is a plausible explanation if energy of the maximum is quantized and requires certain time to collapse to lower energy level. Indeed, if one assumes that fusion in the Sun started with the moment of deceleration when speed became equal to $368 \mathrm{~km} / \mathrm{s}$ and assuming at that point real mass (fusion fuel) was equal to the mass of the [surface] gravitational maximum $\left(1.988500 * 10^{30} \mathrm{~kg}\right)$ it would be reasonable to assume that collapse would occur once energy lost with fusion becomes equal to acquired kinetic mass $(\Delta M)$.

Since this mass has not yet been depleted, the collapse has not occurred yet, but, according to my calculations (see chapter Quantization of the Sun: Energy replenishment), this moment should be near.

The other possibility is that accumulated energy does correspond to current speed $(368 \mathrm{~km} / \mathrm{s})$, but the mass of Neptune has been decreased instead, from $1.08 * 10^{26} \mathrm{~kg}$ to $1.02 * 10^{26} \mathrm{~kg}$ (current mass). Explanations for this may include mass oscillation, moon creation and conversion of gravitational potential to electro-magnetic potential, however, I consider this possibility unlikely. 
Comparing masses of systems of different scales requires proper relativistic treatment. Apart from the speed of light being different between the scales, a proper reference frame must be chosen. In case of comparison of $\mathrm{U}_{1}$ scale system (such as the Solar System) with an $\mathrm{U}_{0}$ system (such as a ${ }^{10} \mathrm{C}$ atom) a proper reference frame is the CMB (Constant Microwave Background) radiation rest frame.

Proper equation is thus (for $\mathrm{v}_{1}=\mathrm{v}_{0}=\mathrm{v}$ ):

$$
\begin{array}{r}
\frac{\text { Sun mass }}{\text { Neptune mass }} \sqrt{1-\frac{v^{2}}{c_{1}{ }^{2}}}=\frac{10 \mathrm{C} \text { nucleus mass }}{10 \mathrm{C} \text { outermost electron mass }} \sqrt{1-\frac{v^{2}}{c_{0}{ }^{2}}} \\
\mathrm{v}=\mathrm{v} \odot=\text { cumulative speed relative to } \mathrm{CMB}=996 \mathrm{~km} / \mathrm{s} \\
\mathrm{c}_{1}=\text { speed of light on } \mathrm{U}_{1} \text { scale }=2.93 * 10^{6} \mathrm{~m} / \mathrm{s} \\
\mathrm{c}_{0}=\mathrm{c}=\text { speed of light on } \mathrm{U}_{0} \text { scale }=2.99792458 * 10^{8} \mathrm{~m} / \mathrm{s}
\end{array}
$$

Note that $\mathrm{CMB}$ radiation is of $\mathrm{U}_{-1}$ scale.

Note also that maximum speed $\left(c_{n}\right)$ depends on pressure and density of space and it is generally not equal to the standard speed of light. Here thus, even though the term speed of light may be used, $\mathrm{c}_{1}$ should be understood as maximum speed of $U_{1}$ particles in flat intergalactic space equivalent to standard $\left(\mathrm{U}_{0}\right)$ vacuum.

Within the galaxy, speed limit for orbiting bodies is generally defined by the gravitational maximum (event horizon) of the well - stars do exist orbiting black holes faster than $\mathrm{c}_{1}$, however, probability for stars moving at speeds $\geq c_{1}$ outside of galaxies is negligible.

One can now attempt to resolve the excess mass of TOI-178 $\left({ }^{6} \mathrm{Be}\right)$ system. Assuming its velocity [relative to CMB] is $77.22 \mathrm{~km} / \mathrm{s}$ larger than Sun's velocity, its mass should be:

$$
\begin{aligned}
M_{T O I-178}=\frac{M_{B e-6}}{M_{C-10}} M \frac{1}{\sqrt{1-\frac{v^{2}}{c_{1}^{2}}}} & =1.207764563 * 10^{30} \mathrm{~kg} \\
& =0.607 M_{\odot}=0.646 \mathrm{M}
\end{aligned}
$$

$\mathrm{M}_{B e-6}=$ rest mass of ${ }^{6} \mathrm{Be}$ atom $=6.0197 \mathrm{u}$ $\mathrm{M}_{C-10}=$ rest mass of ${ }^{10} \mathrm{C}$ atom $=10.016853 \mathrm{u}$

$\mathrm{M}=$ rest mass of the Sun (relative to CMB) $=1.870062271 * 10^{30} \mathrm{~kg}$ $\mathrm{v}=$ cumulative speed of TOI-178 relative to $\mathrm{CMB}=1073.22 \mathrm{~km} / \mathrm{s}$

However, mass of TOI-178 obtained from measurements is $0.650^{+0.027 /-0.029} \mathrm{M}_{\odot}[7]$. 
Apparently, measured mass is bigger by the relativistic [omega] factor:

$$
\frac{1}{\sqrt{1-\frac{v^{2}}{c_{1}^{2}}}} \approx \frac{v}{v_{\odot}}
$$

The cause of discrepancy is, again, in the reference frame - calculation is done relative to CMB, while measurements were done from the Solar System (Earth) reference frame.

From such reference frame Sun is at rest and its rest mass is equal to relativistic mass relative to $\mathrm{CMB}, \mathrm{M}_{\odot}\left(1.988500 * 10^{30} \mathrm{~kg}\right)$.

However, one must take into account the radial velocity [relative to the Sun] of TOI-178. Relative to the Solar System, the mass of TOI-178 should thus be:

$$
\begin{aligned}
M_{T O I-178} & =\frac{M_{B e-6}}{M_{C-10}} \frac{M}{\sqrt{1-\frac{\left(v_{\odot}+v_{r}\right)^{2}}{c_{1}{ }^{2}}}} \frac{1}{\sqrt{1-\frac{v^{2}}{c_{1}{ }^{2}}}} \\
\mathrm{v}_{r} & =\text { radial velocity of TOI- } 178=57.4 \pm 0.5 \mathrm{~km} / \mathrm{s}
\end{aligned}
$$

This gives $0.650 \mathrm{M}_{\odot}$ for the mass of TOI-178, in agreement with measurements.

Note that relativistic effects are always physical, but not always on the same scale and not always in the same space - ie. some may be physical on small scale (mental) in space of the observer, some on a large scale in space of the observable, or vice versa[8].

Solar System is thus a [negatively] polarized reference frame relative to TOI-178 and to convert the measurement to a proper [neutral] reference frame, one must multiply the measured value with a positively polarized omega factor:

$$
\left(\frac{1}{\sqrt{1-\frac{\left(v_{\odot}+v_{r}\right)^{2}}{c_{1}{ }^{2}}}}\right)^{-1}=\sqrt{1-\frac{\left(v_{\odot}+v_{r}\right)^{2}}{c_{1}{ }^{2}}}
$$

\subsection{EH operator validation}

The following is an attempt to validate the EH operator defined in CR. However, this is completely unnecessary for validation of CR or further analysis of the Solar System. 
Graviton and photon mass have already been calculated in CR from predicted discrete vertical energy levels. Agreement with other calculations and experimental data show that Neptune is a vertically excited electron. This is an alternative calculation of these masses.

If the carbon atom at appropriate density/pressure is the Solar System, carbon photon is the carbon atom of lower scale (vertical energy level).

One can thus calculate the [average] mass of photons or photon scale particles, ie. electron photon:

$$
\frac{\text { Neptune mass }}{10 \mathrm{C} \text { outermost electron mass }}=\frac{10 \mathrm{C} \text { outermost electron mass }}{\text { e photon mass }}
$$

e photon mass $=\frac{(10 \mathrm{C} \text { outermost electron mass })^{2}}{\text { Neptune mass }}=8.102214736 * 10^{-87} \mathrm{~kg}$

However, obtained photon mass above assumes linear progression of discrete states of scale invariance (vertical symmetry, distance in scale from $\mathrm{U}_{0}$ to both $\mathrm{U}_{1}$ and $\mathrm{U}_{-1}$ is equal), which is against the postulates of $\mathrm{CR}$ - although this can be the mass of a photon in another time (another cycle state).

There can be no symmetry between current space and time, but due to cyclic nature of a universe and with cycle states being inverse of each other, symmetry would exist between past and future dimensions (space and time dimensions exchange in a way that current space is symmetric with previous space).

Thus, CR predicts asymmetric invariance with exponential progression of discrete vertical states. Using this prediction, the masses of standard photon [scale] electron equivalent (half-photon) and carbon graviton neutrino have been calculated already in CR (yielding $9.10938356 * 10^{-73} \mathrm{~kg}$ for the photon e mass, $1.663337576 * 10^{-68} \mathrm{~kg}$ for the half-neutrino mass), but the values can also be obtained using EH operator.

Using EH factor 6/4 on the orders of magnitude of mass distances:

$$
\log _{10}\left(\frac{M_{U_{1}}}{M_{e}}\right)=E H_{6 / 4}\left[\log _{10}\left(\frac{M_{e}}{M_{n}}\right), \log _{10}\left(\frac{m_{U_{1}}}{M_{n}}\right)\right]
$$

gives $\mathrm{M}_{n}=3.910613743 * 10^{-68} \mathrm{~kg}$ for the mass of neutrino in current cycle state, and $\mathrm{m}_{U_{1}}=6.06011796 * 10^{19} \mathrm{~kg}$ for the initial real mass of Neptune in current cycle state (or, its properly scaled mass - relative to its gravitational maximum). 
Here, $\mathrm{M}_{p}=\mathrm{M}_{n} / \mathrm{m}_{U_{1}}=6.453032383 * 10^{-88} \mathrm{~kg}$ could be interpreted as the mass of carbon photon in inverse cycle state.

Mass of a photon can now be obtained from Mn:

$$
M_{p}=\frac{M_{e}}{{ }^{10} C \text { atom mass }} M_{n} \approx M_{n} * 10^{-5}
$$

Note that, in current state the ratio of magnitude distances from electron to neutrino and from electron to $\mathrm{U}_{1}$ electron (Neptune) is:

$$
\log _{10}\left(\frac{M_{e}}{M_{n}}\right)\left[\log _{10}\left(\frac{M_{U_{1}}}{M_{e}}\right)\right]^{-1}=\frac{4}{6} \frac{5}{5}=\frac{2}{3}
$$

So, for the inverse state $(4 \mathrm{p} 6 \mathrm{n})$ :

$$
\begin{gathered}
\log _{10}\left(\frac{M_{e}}{M_{n}}\right)\left[\log _{10}\left(\frac{M_{U_{1}}}{M_{e}}\right)\right]^{-1}=\frac{6}{4} \frac{3}{7}=\frac{9}{14} \\
\log _{10}\left(\frac{M_{U_{1}}}{M_{e}}\right)=E H_{4 / 6}\left[\log _{10}\left(\frac{M_{e}}{M_{n}}\right), \log _{10}\left(\frac{m_{U_{1}}}{M_{n}}\right)\right]
\end{gathered}
$$

Respecting conditions for the $\mathrm{EH}$ inverse, the following values are obtained: mass $\mathrm{M}_{e}=3.910613743 * 10^{-68} \mathrm{~kg}$ of $\left[{ }^{10} \mathrm{C}\right.$ outermost $]$ electron equivalent in $\mathrm{U}_{-1} .4 \mathrm{p} 6 \mathrm{n}\left(=\mathrm{M}_{n}\right.$ in $\left.\mathrm{U}_{0} \cdot 6 \mathrm{p} 4 \mathrm{n}\right), \mathrm{M}_{U_{1}}=9.10938356 * 10^{-31} \mathrm{~kg}$ for the mass of Neptune equivalent in $\mathrm{U}_{-1} .4 \mathrm{p} 6 \mathrm{n}\left(=\mathrm{M}_{e}\right.$ in $\left.\mathrm{U}_{0} .6 \mathrm{p} 4 \mathrm{n}\right), \mathrm{M}_{n}=3.719162593 * 10^{-92}$ $\mathrm{kg}$ for the mass of neutrino in $\mathrm{U}_{-1} .4 \mathrm{p} 6 \mathrm{n}, \mathrm{m}_{U_{1}}=4.18129939 * 10^{-36} \mathrm{~kg}$ for the real mass of Neptune in $\mathrm{U}_{-1} .4 \mathrm{p} 6 \mathrm{n}\left(=\mathrm{m}_{e}\right.$ in $\left.\mathrm{U}_{0} \cdot 6 \mathrm{p} 4 \mathrm{n}\right)$.

Note that here, mass of the photon is obtained from:

$$
M_{p}=\frac{{ }^{10} \mathrm{C} \text { atom mass }}{M_{e}} M_{n}=6.791044478 * 10^{-88} \mathrm{~kg}
$$

suggesting inverted roles of photon and neutrino.

\subsection{Outermost angular momenta and $\mathrm{c}_{1}$ confirmation}

With the conservation of angular momentum between the Solar System equivalent at $\mathrm{U}_{0}$ scale $\left({ }^{10} \mathrm{C}\right.$ atom at equivalent density/pressure) and the Solar System, one may attempt to calculate angular velocity of the outermost electron in the ${ }^{10} \mathrm{C}$ atom:

$$
L=m v r=\frac{v}{r} m r^{2}
$$




$$
\begin{gathered}
M_{U_{1}} v_{U_{1}} r_{U_{1}}=M_{U_{0}} v_{U_{0}} r_{U_{0}} \\
v_{U_{0}}=\frac{M_{U_{1}} v_{U_{1}} r_{U_{1}}}{M_{U_{0}} r_{U_{0}}}=3.920242676 * 10^{82} \frac{\mathrm{m}}{\mathrm{s}}
\end{gathered}
$$

The above gives the outermost electron velocity in case of conversion of both mass and orbital radius into angular velocity, for a point energy in constant vacuum density.

However, mass $\mathrm{M}_{U_{0}}$ must have been relativistic before the speed limit was reached (vertical energy level changed) and it became the rest mass $\mathrm{M}_{U_{1}}$.

Thus, in order to get the orbital velocity just before the [vertical] energy level change, rest mass on one scale must be equalized with relativistic mass on another $\left(\mathrm{M}_{U_{1}}=\mathrm{M}_{U_{0}}\right)$ :

$$
v_{U_{0}}=\frac{v_{U_{1}} r_{U_{1}}}{r_{U_{0}}}=3.486882257 * 10^{26} \frac{\mathrm{m}}{\mathrm{s}}
$$

With real mass not participating in inflation (maxima inflate naked), this velocity is the velocity of space, making it valid even in the context of General Relativity (GR).

Using conservation of energy, one can now obtain the velocity of the outermost electron in standard non-excited ${ }^{10} \mathrm{C}$ atom:

$$
\begin{gathered}
E_{-1}=E_{0} \\
\rho_{v a c} * V_{U_{0}} * v_{U_{0}}{ }^{2}=M_{U_{0}} * v^{2} \\
\rho_{v a c}=\text { mean vacuum energy density }=9.9 * 10^{-27} \frac{\mathrm{kg}}{\mathrm{m}^{3}} \\
\rho_{v a c} * \frac{4}{3} \pi\left(R_{U_{0}}\right)^{3} * v_{U_{0}}{ }^{2}=M_{U_{0}} * v^{2} \\
2.842208873 * 10^{-19}=M_{U_{0}} * v^{2}
\end{gathered}
$$

This gives $\mathrm{v}=5.585837356 * 10^{5} \mathrm{~m} / \mathrm{s}$, for the velocity of the outermost electron of a standard ${ }^{10} \mathrm{C}$ atom [in Solar System equivalent state].

Note that the product of density and volume on the left should be the mass of a standard photon $\left(2.337660431 * 10^{-72} \mathrm{~kg}\right)$, and it is indeed roughly equal to previously calculated photon rest mass in CR $\left(1.821876712 * 10^{-72} \mathrm{~kg}\right)$.

Using momentum conservation, one can now calculate photon mass relative to standard (absolute) reference frame, where its speed is limited 


$$
\begin{gathered}
\text { to } \mathrm{c}=\mathrm{c}_{0}=2.99792458 * 10^{8} \mathrm{~m} / \mathrm{s}: \\
p=m v=m v_{U_{0}}=2.337660431 * 10^{-72} \mathrm{~kg} * 3.486882257 * 10^{26} \frac{\mathrm{m}}{\mathrm{s}}=m_{0} c_{0} \\
m_{0}=\frac{p}{c_{0}}=\frac{p}{c}=2.719 * 10^{-54} \mathrm{~kg}
\end{gathered}
$$

or, using photon rest mass from CR:

$$
m_{0}=2.119 * 10^{-54} \mathrm{~kg}
$$

This mass is in agreement with photon mass obtained from recent experiments[9].

To confirm validity of the result one can calculate this velocity differently. Introducing the term total velocity $\left(\mathrm{v}_{t o t}\right)$ as the sum of electron's spin and angular velocity.

Per CR postulates, every spin momentum must be an orbital momentum. If one assumes that, once captured by the atom, the outermost electron selforbital (spin) momentum becomes the nucleus-orbital momentum, in ground state (with quantum number $1=0$ ) thus, total momentum of the electron is:

$$
\begin{gathered}
m r^{2} \omega_{t o t}=\frac{1}{2} \hbar \\
v_{t o t}=r \omega_{t o t}=\frac{1}{2} \frac{\hbar}{m r}
\end{gathered}
$$

Using $\mathrm{m}=\mathrm{M}_{U_{0}} \approx \mathrm{M}_{e}$ and $\mathrm{r}=\mathrm{r}_{U_{0}}$, this gives $\mathrm{v}_{\text {tot }}=8.269308487^{*} 10^{5} \mathrm{~m} / \mathrm{s}$. This momentum in the atom is further divided between orbital and spin momentum. With the ratio of velocities equal to Neptune spin/orbital velocity, one obtains electron orbital velocity:

$$
v=v_{U_{0}}=\frac{v_{t o t}}{1+\frac{s_{U_{1}}}{v_{U_{1}}}}=5.5550351679 * 10^{5} \frac{\mathrm{m}}{\mathrm{s}}
$$

The result is obtained from the following:

$$
\begin{gathered}
v_{t o t}=v_{a}+v_{s} \\
M_{e} v_{t o t} r_{a}=\frac{1}{2} \hbar
\end{gathered}
$$

Splitting the momentum in scalar space:

$$
m_{r e} v_{a} r_{a}+m_{i m g} v_{s} r_{s}=M_{e} v_{t o t} r_{a}
$$




$$
\frac{m_{r e}}{M_{e}} v_{a}+\frac{m_{i m g}}{M_{e}} v_{s} \frac{r_{s}}{r_{a}}=v_{t o t}
$$

and assuming:

$$
m_{r e}=M_{e}
$$

from Q1.2 and Q1.4, follows:

$$
m_{i m g}=M_{e} \frac{r_{a}}{r_{s}}
$$

$\mathrm{M}_{e}=$ standard electron mass $=9.10938356 * 10^{-31} \mathrm{~kg}$ $\mathrm{r}_{a}=\mathrm{r}_{U_{0}}=$ orbital radius of the outermost ${ }^{10} \mathrm{C}$ electron $=70 * 10^{-12} \mathrm{~m}$ $\mathrm{r}_{s}=\mathrm{R}_{U_{0}}=$ spin radius of the outermost ${ }^{10} \mathrm{C}$ electron $=3.834298096{ }^{*}$ $10^{-16} \mathrm{~m}$

In order for Q1.2 to be satisfied, masses of orbital and spin momenta must be different. With orbital mass equal to standard electron mass, spin mass $\mathrm{m}_{\text {img }}$ is:

$$
\begin{gathered}
m_{i m g}=1.66303410 * 10^{-25} \mathrm{~kg}=9.99817551 *{ }^{10} \mathrm{C} \text { nucleus mass } \\
m_{i m g} \approx 10 *{ }^{10} \mathrm{C} \text { nucleus mass } \approx 93.3 \mathrm{GeV} / \mathrm{c}^{2}
\end{gathered}
$$

Note that the increase in electron spin mass $\mathrm{m}_{i m g}$ is proportional to the increase of nucleus mass. In both, mass component of the spin momentum was increased at the expense of other components, as with electromagnetic coupling the em energy was converting to neutral gravitational energy. Note also that, from this, it is possible to derive the rest mass and rest charge radii of a free electron. Assuming radius inflation proportional to mass inflation, rest mass radius of a free electron is:

$$
r_{s_{e}}=\frac{r_{s}}{r_{a}} r_{s}=\frac{r_{s}^{2}}{r_{a}}=2.100 * 10^{-21} \mathrm{~m}
$$

Its rest charge radius should then be:

$$
r_{c_{e}}=\sqrt{2} r_{s_{e}}=2.970 * 10^{-21} \mathrm{~m}
$$

Obviously the charge of the electron has to be spinning faster than light:

$$
v=\frac{1}{2 m r_{c_{e}}} \hbar
$$

For $\mathrm{m}=9.10938356 * 10^{-31} \mathrm{~kg}$ (which may seem wrong due to separate mass radius, however, if free electron is not naked, acquired real mass can be the charge mass shielding the mass of the maximum), this gives $\mathrm{v}=9.745 * 10^{15} \mathrm{~m} / \mathrm{s}$. 
This speed is the speed limit for particles in electron's space and it suggests that acquired real mass is of $\mathrm{U}_{-1}$ scale or lower, making the spin momentum of the electron effectively the rotation of space, relative to standard scale. The fact that imaginary mass is quantized by ${ }^{10} \mathrm{C}$ nucleus mass confirms the carbon-like nature of the Solar System equivalent on the standard scale, however, the magnitude of exchange of polarized (electro-magnetic) potential for neutral gravitational potential suggests the Solar System is a scaled Bose-Einstein condensate of multiple atoms. Note that the mass is equal to predicted $\mathrm{W}$ boson mass in some Electroweak models[10].

From the calculated mass one can now obtain real part of Neptune's total mass:

$$
\begin{gathered}
\frac{m_{r e}}{m_{i m g}}=\frac{m_{r e_{1}}}{m_{i m g_{1}}} \approx \frac{m_{r e_{1}}}{M_{U_{1}}} \\
m_{r e_{1}}=\frac{M_{e}}{m_{i m g}} M_{U_{1}}=5.60974244 * 10^{20} \mathrm{~kg}
\end{gathered}
$$

In the above, it was assumed that charge radius is equal to mass spin radius $\left(\mathrm{r}_{s}\right)$ of the gravitational maximum. However, real charge radius is smaller.

If one assumes Earth's mass radius of the gravitational maximum is at the inner core boundary with gravity equal to Sun surface gravity (274 $\mathrm{m} / \mathrm{s}^{2}$ ), charge radius of Earth must be at a radius where gravity of the maximum is equal to half this value (this will be validated later):

$$
\begin{gathered}
r_{c}=\sqrt{G M \frac{2}{274}}=\sqrt{\frac{G M}{137}}=1705704 m \\
\mathrm{M}=\text { Earth's mass }=5.9723^{*} 10^{24} \mathrm{~kg} \\
\mathrm{G}=\mathrm{G}_{0}=\text { standard gravitational constant }=6.674 * 10^{-11} \mathrm{~m}^{3} / \mathrm{kgs}^{2}
\end{gathered}
$$

Using Q1.5, one can now calculate real mass component of the Earth:

$$
\begin{aligned}
m_{r e}=\frac{r_{c}}{r_{a}} m_{i m g} & \approx \frac{r_{c}}{r_{a}} M \approx 6.81 * 10^{19} \mathrm{~kg} \\
\mathrm{r}_{a} & =\text { Earth's orbital radius }=149.6 * 10^{9} \mathrm{~m}
\end{aligned}
$$

This real mass will be further validated later. However, obtained charge radius is, as it will be shown later, induced charge radius, rather than the primary or primordial charge radius.

Calculating $\mathrm{m}_{\text {img }}$ for other planets shows weak signals that all may be consistent with condensates of standard particles, as shown in Table 2 . 


\begin{tabular}{l|l|r} 
planet & $\begin{array}{l}\text { equivalent standard } \mathrm{m}_{i m g} \\
\left(\mathrm{GeV} / \mathrm{c}^{2}\right)\end{array}$ & particle \\
\hline Saturn & 12.58 & $10 *$ charm quark, or $2 *$ charmed B meson \\
Jupiter & 5.69 & ADM (asymmetric dark matter) particle ? \\
Uranus & 57.87 & 10 * ADM ? \\
Mars & 34.36 & $?$ \\
Earth & 12 & $?$ \\
Venus & 9.14 & $?$ \\
Mercury & 12.13 & $?$
\end{tabular}

Table 2: Calculated $\mathrm{m}_{\text {img }}$ and standard particle candidates

There are no obvious candidates for terrestrial planets, and excluding Saturn and Neptune, even the candidates for outer planets may be questionable.

However, there are various reasons for this - mass oscillation/excitation, unusual pairing (as in case of Neptune), unknown particle (having extremely short lifetimes on standard scale, unstable outside of atom), etc.

Multiples of atomic nuclei have also not been taken into account, which may be the most likely candidates, given the strong agreement of quantization of outermost particle mass with ${ }^{10} \mathrm{C}$ nuclei and the fact that, in a condensed state, an atomic nuclei is a viable equivalent of a particle (quantum of energy).

Two results for the velocity are in good agreement. Small difference can be attributed to uncertainty in vacuum energy density - a value of $9.79 * 10^{-27}$ $\mathrm{kg} / \mathrm{m}^{3}$ would yield the correct value.

Note that properties of space change inside the atom, this includes $\epsilon_{0}$ and, consequently, $\hbar$ constant. Thus, velocities calculated using QM are generally not real (this velocity would produce a $1 / 3 \hbar$ orbital angular momentum in QM).

That properties of space change within the atom is obvious through the varying speed of light between different materials. Relative dielectric and magnetic constants should be interpreted as evidence for this - photon can get absorbed and re-emitted in the atom, but what causes the delay? If electrons have different energies on different orbits but have equal rest mass, energy must be stored in local space, varying with distance. If spin of the electron changes, energy is stored in its own space. All of these spaces are quantized, but using the same constants for all of them will generally not produce results matching reality. It may provide good and consistent results when measuring effects outside of the atom in consistent external space, but it gives a wrong image of its internal equilibrium structure.

From this one can also obtain the scaled speed of light:

$$
\begin{gathered}
\frac{v_{U_{0}}}{c_{0}}=\frac{v_{U_{1}}}{c_{1}} \\
c_{1}=\frac{v_{U_{1}}}{v_{U_{0}}} c_{0}=2.930445979 * 10^{6} \frac{\mathrm{m}}{\mathrm{s}}
\end{gathered}
$$


The result is in agreement with $c_{1}$ previously obtained from kinetic energy of the Solar System $\left(2.93 * 10^{6} \mathrm{~m} / \mathrm{s}\right)$.

\section{$6 \quad$ Initial setup and regular disturbances}

Solar System is the product of inflation (likely through annihilation) of smaller scale particles or/and deflation [through annihilation] of larger scale particles.

Suppose that at the moment of annihilation the carbon atom was briefly ionized and its mass and charge were condensed into the core when it started inflating. With the electrons inflating along, eventually, the charge would separate from mass again.

The energy provided for transition between adjacent energy levels is generally higher than required, thus, the flattened carbon atom likely expanded to multiple times its current radii, then compressed to current size, trading charge area for neutral gravitational volume.

The atom nucleus in the process expanded up to the main asteroid belt, then compressed, leaving behind orbiting gravitons which collapsed to form terrestrial planets. The collapses were recorded in the Sun, forming discontinuities.

Note that the effect is the same even without initial ionization - in that case, discontinuities would be inflated along with the atom, rather than produced in the process.

In the transition from charged two-dimensional ring to three-dimensional sphere, equatorial spin momentum has been fragmenting and [due to spin decoupling] spreading to (forming) polar regions.

Latitude variable rotation may have been initially established as the product of conservation of momentum in such redistribution of mass, even if it now may be sustained differently.

Beside the long lived energy level changes, short lived (temporary) inflation/deflation of gravitational maxima will occur with the absorption/emission of [properly scaled] gravitational waves, which may be electrically polarized (electro-magnetic).

In case of dipole waves, absorption will induce separation of charges and collapse of a spherical form of the maximum into a two-dimensional ring form.

Such disturbances will generally occur at regular intervals, with periods generally increasing proportionally to the scale of the system and the scale of disturbance. On the scale of stellar systems, common minimum periods are on the order of millions of years. 
Changes in energy of the Solar System cannot be exempt from general oscillation and remain uniform over its lifetime.

For the Solar System, I hypothesize the existence of 3 periods (the evidence for which will be provided later, in this article, and in follow-up articles) for the first three orders of general oscillation:

1. $4.25 * 10^{9}$ years.

2. $25.7-25.92 * 10^{6}$ years,

3. $1.512 * 10^{6}$ years,

These are cycles of existence of the Solar System and its bodies.

Only the 1st order cycle may result in large scale horizontal energy level changes, but all these disturbances are sourced in gravitational stresses and have a strong effect on the evolution of the system (and all life within) - it is temporarily accelerated at the end of each cycle.

1st order period should be interpreted as lifespan of the Solar System as a whole. At time of death, gravitational maximum(s) of the Sun [and likely all planets] collapse exchanging spin momenta for galactic angular momenta. Eventually, this system may couple with real mass and inflate again into the same species (carbon in this case). It may even couple with the same mass, in which case the collapse may be interpreted as temporary loss of consciousness.

It may also inflate or deflate through annihilation or fusion with another system, and then start evolving as new life form of another generation or new species, acquiring real mass in vicinity.

In any case, life and death are synchronized, and, for these species death is likely not the same as death on our scale. Discarded real mass may be fully reused by another soul in these species - with no temporary and/or spatially large decay and recycling involved.

2nd order period should be interpreted as the lifespan of Sun's core and Jupiter, and possibly all outer planets. Based on current evidence, these collapses should be temporary regardless of nature (death or loss of consciousness). Naturally, these collapses will cause orbital disturbances, and are likely to induce bombardment of terrestrial planets with asteroids and comets.

These should thus be correlated with large extinctions on these planets.

1st order period should be interpreted as the lifespan of Earth and possibly all inner (terrestrial) planets (at least in the order of magnitude). Based on evidence, this collapse too is temporary.

Collapse of Earth's maximum will be synchronized with accelerated evolution of life on its surface. There is evidence for accelerated human 
evolution $1.4-1.6 \mathrm{Ma}[11]$. Thus, another such event (effective time compression) is likely happening right now.

All of these periods are time averaged, deviations will exist, but larger periods should be relatively quantized by [and thus, relatively synchronized with] smaller periods.

Ongoing extinction on Earth may be correlated with the end of a 3rd order period, however, everything suggests this is also the end of a 2nd order period. And, considering the age of Earth and the Solar System, we are likely at the end of a 1st order period too. Thus, major cataclysmic changes should be imminent, although, there is some possibility that 1st order period will end with the end of an additional 2nd order period, some 26 million years later.

Note that currently accepted age of Earth and the Solar System, based on uniform evolution and decay rates of elements, must be wrong. Decay rates of elements cannot be constant over all time, they must change, either directly with changes in pressure and density of space, or effectively - ie. with cosmic ray bombardment. Thus, they should temporarily change (accelerate) with the end of a period of any order - proportionally to order period and acceleration in evolution.

Large scale events are always preceded and superseded by smaller scale events so accelerated evolution may proceed for years on smaller scales before the actual disruption on larger scale occurs.

One may now attempt to calculate how much such disturbances last on the large (cataclysmic) scale.

With no change in energy level, orbital areal velocity of bodies, per Kepler's 2nd law, must remain constant and there should be no change in constitutional mass either.

With a temporary collapse of a gravitational maximum, escape velocity is extremely reduced and orbiting neutral real mass will be increasing orbital radii (although solid mass will generally preserve volume due to smaller scale electromagnetic and neutral gravitational forces).

In order for this to be a temporary disturbance (no loss of entanglement), collapse must not exceed a specific time period - orbital period of the constituting mass.

Approximating gravitational maximum as a point maximum (linear ejection of mass from center) and assuming Sun's constitutional mass barycenter at the [inner] core radius at the time of collapse of the Sun's core maximum, maximal allowed ejection distance $r$ at the time the gravitational well is fully restored is:

$$
\begin{aligned}
& r=\frac{2 \pi r_{c}}{2}=\pi r_{c} \approx 0.63 R_{\odot} \\
& \quad \mathrm{R}_{\odot}=\text { Sun radius }=695700 \mathrm{~km} \\
& \mathrm{r}_{c}=\text { inner core radius }=1 / 5 \mathrm{R}_{\odot}=139140 \mathrm{~km}
\end{aligned}
$$


Maximum time between the collapse and full restoration of the well is then:

$$
t_{c}=\frac{2 \pi r_{c}}{v_{c}}=\frac{1}{f_{c}}=608272.5061 \mathrm{~s} \approx 7 \text { days }
$$

where $\mathrm{f}_{c}(1644 \mathrm{nHz}[12])$ is the rotation frequency of the Solar core.

Note that there is a discontinuity in the seismic profile of the Sun at $0.63 R_{\odot}$. This is where Sun's angular velocity starts differentiating with latitude (it rotates as a solid from $0.63 \mathrm{R}_{\odot}$ down to the core).

Note also the following:

$$
\begin{aligned}
\frac{1}{v_{c}} * 10^{12}=\frac{1}{2 \pi r_{c} f_{c}} * 10^{12} & =\frac{1}{2 \pi * 0.2 * 695700 * 1644 * 10^{-9}} * 10^{9} \\
& =695771 \mathrm{~km} \approx R_{\odot}
\end{aligned}
$$

suggesting that this should be satisifed:

$$
v_{c} * R_{\odot}=1 * 10^{12} \frac{\mathrm{m}^{2}}{\mathrm{~s}}
$$

or, in terms of areal velocity of the core:

$$
v_{a}=\frac{1}{2} v_{c} r_{c}=\frac{R_{\odot}^{2} \pi}{5^{2} t_{c}}=1 * 10^{11} \frac{\mathrm{m}^{2}}{\mathrm{~s}}
$$

A hint of deeper entanglement between the Solar core and surface maximum (quantization).

In the context of CR, evolution of systems is not a steady continuous process over all time, but a process with cyclic strong (cataclysmic) changes and a slow (weak) continuous evolution through the cycle.

\section{Effects of mass and gravitational stresses on Keplerian motion}

Orbits of bodies in gravitationally bound systems should obey the following equation (orbital law):

$$
v^{2}=\frac{G M}{r} \quad \mathrm{G}=\text { gravitational constant }
$$

where $\mathrm{v}$ and $\mathrm{r}$ are orbital (Keplerian) velocity and radius, respectively, while $\mathrm{M}$ is the mass contained within the radius $\mathrm{r}$. 
In planetary systems, most of the mass $M$ is contained within the star, while in galaxies, it is mostly in central supermassive black holes.

However, in both systems, there are orbits at which the equation is apparently not satisfied - $\mathrm{v}$ is either higher or lower than expected for detected mass M.

In galaxies, it is assumed that the discrepancy is caused by exotic gravitational mass - dark matter.

In planetary systems, spin of bodies does not obey the equation, but this is largely ignored (not considered as discrepancy), possibly due to current understanding of gravity and accepted theories on formation of planetary systems.

It is however, a legitimate question - why should a gravitationally bound mass in a galaxy obey the orbital law, while clouds of gas orbiting near the surface of a star should not (if most of $M$ is below the surface)?

If the problem of gas is ignored because it is considered as constituent mass of the star, why are stars themselves not considered as constituent part of galaxies? And why would all constituent mass be exempt from the orbital law in the first place?

In CR, gravitational force of bodies with a distinct gravitational well may be largely provided by the gravitational maximum(s) so [ordinary] matter content (real mass) may be low.

Thus, a potential equivalent dark matter problem may exist in stars, planets, dwarf planets and larger moons (asteroids and comets are composites of smaller scale wells [held together in most part by electro-magnetic force] so their spin momentum should not be Keplerian, even if their orbit around a body with a distinct maximum should).

All bodies with a distinct gravitational well have a dark matter source (gravitational maximum), however, the addition (acquisition) of smaller scale matter (real mass) will shield the existence of the maximum, effectively decreasing imaginary mass content of the well.

Note that, in this exchange of dark gravitational potential for real gravitational potential, net gravitational force remains constant, but the capacity of the well (for real mass) is decreasing.

A body may also have multiple maxima, in which case, the outermost (surface) maximum will shield the existence of deeper maxima.

The shielding effect is not limited to the neutral gravitational component of general force, electro-magnetic component may be shielded as well. Thus, if there is no exchange of neutral gravitational potential for electromagnetic potential, and if there are no changes in kinetic energy, despite the loss of matter, the gravity of a star should not change with age. The 
attraction remains, but its nature changes - from being mostly in its looks (real mass) to being mostly in its spirituality (dark matter), as in any living being.

Luminosity is thus, generally, a good measure of gravitational mass only if the well is at full capacity, otherwise it is only correlated with real mass, and age (if there is no fuel replenishment).

However, even if real mass may not be correlated with total gravity at all times, these should get synchronized periodically. The reason why they are not synchronized at all times may simply be a difference in scale - since energy changes are discrete, burning of real mass (small scale mass) will appear continuous, while on large scale, where energy quanta are orders of magnitude bigger, change in mass (gravity) may require millions or billions of years.

It is thus possible that the Sun does not have much fuel (real mass) left at this point, its gravity is rather in dark matter associated with the maximum that is yet to collapse.

It is then likely that a collapse is synchronized with depletion of fusion fuel.

The solution for terrestrial bodies lies in the loss of entanglement between space and matter orbitals due to interaction (collision) with other bodies, during formation of the body of matter.

Due to interaction of the atmosphere with a solid body beneath (or its origin), neither the gases of the atmosphere (or trapped particles from outer space interacting with the atmosphere) may obey the orbital law.

This suggests that even below a gas cloud rotating around a distinct maximum at non-Keplerian velocity there should be a solid core, at least in case of a neutral gas, however, angular component of velocity may be converted to radial and then to temperature.

Note that even if pressure from high temperature (kinetic energy) is balancing gravitational force, the thermodynamics (within the gas cloud) cannot break the orbital entanglement of the gas cloud as a whole.

If that gas is in the form of plasma (as in the case of Sun), it is more likely to be entangled with the charge component of a maximum (general force), which then should be the source of its non-Keplerian motion.

The neutral gravitational equivalent of electro-magnetic influence on gas 
on the equator of the Sun can be calculated:

$$
\begin{array}{r}
v=v_{e}=\frac{2 \pi r}{T}=\sqrt{\frac{G M_{2}}{r}}=2066.95 \frac{\mathrm{m}}{\mathrm{s}} \\
\mathrm{v}_{e}=\text { equatorial velocity of the Sun surface } \\
\mathrm{G}=\text { gravitational constant }=6.674 * 10^{-11} \mathrm{~m}^{3} / \mathrm{kgs}^{2} \\
\mathrm{r}=\text { equatorial radius of the Sun }=695500 \mathrm{~km} \\
\mathrm{~T}=\text { rotation period at equator }=24.47 \text { days }
\end{array}
$$

which gives for the mass of the hypothetical neutral maximum:

$$
M_{2}=4.45215 * 10^{25} \mathrm{~kg}
$$

If the electro-magnetic component of the maximum would be exchanged for neutral gravitational component, the equatorial matter could remain entangled with such maximum.

The observed angular velocity could be interpreted as the evidence of spin change during the transition between vertical energy levels and transformation of electro-magnetic potential for neutral gravitational potential. Suppose that entire potential was initially electro-magnetic but with an opposite spin. During transformation, Keplerian velocity component would be decreasing total angular velocity and, as the neutral component becomes larger than the electro-magnetic component, real mass would start spinning in another direction - aligned with Keplerian velocity. With complete transformation, real mass would have a Keplerian angular velocity.

However, with the exchange of potential and inflation of space, [assuming real mass is acquired not inflated] increasing gravity must be radially compressing orbitals, increasing density of real mass. If the compression is not isotropic and the mass is spiraling inwards (as expected for interaction of binaries at the event of annihilation), angular velocity (being exchanged for radial) will be decreasing from Keplerian with orbital radius.

This will be increasing pressure and temperature around the center which will balance the neutral gravitational force at equilibrium.

Angular velocity of matter around stars is thus generally proportional to a difference between neutral and electro-magnetic potential and, in magnitude, inversely proportional to temperature/density of real mass.

However, stability of a gravitational maximum is proportional to its mass and inversely proportional to gravitational stress.

That gravitational stress affects the number of sunspots has already been shown[13], and here I hypothesize that a sunspot pair is the result of a collapse of a quantum of a neutral gravitational surface maximum into a pair of [electrically] 
oppositely charged and relatively unstable smaller (spin) maxima.

Note that orbital radius of a sunspot pair is equal to the radius of the maximum before collapse.

Gravitational wells of planets, dwarf planets and major moons have been formed in the similar way as sunspots.

Note also that size of sunspots ranges from the size of a moon to the size of the biggest planet (Jupiter).

A neutral component of a naked gravitational maximum is gravitational energy that may be referred to as dark matter, visible matter is real mass attracted to the gravitational well of such maximum. The velocity curves of the Sun and the Milky Way galaxy likely have the same solution - in the form of gravitational maxima and relativity of their nature due to exchange between polarized and non-polarized potentials of general force.
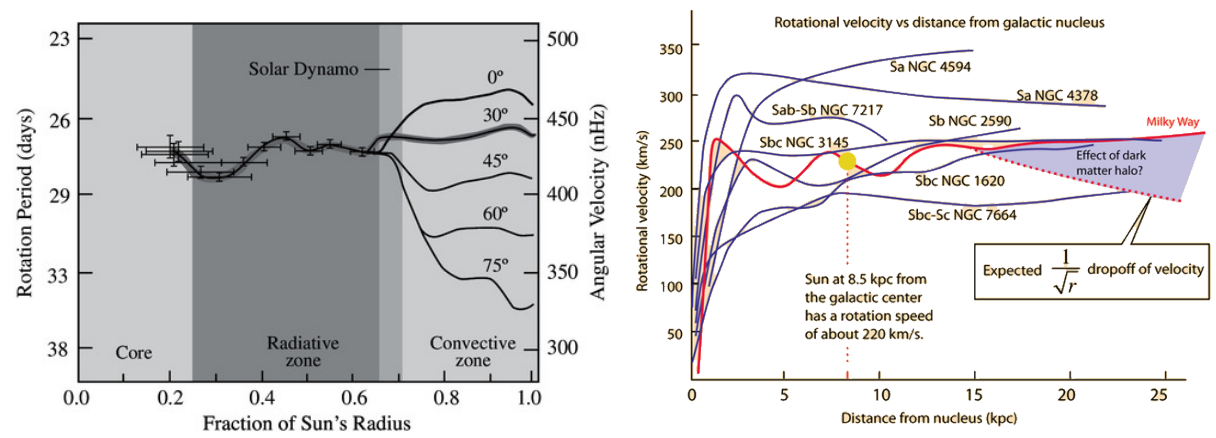

Figure 7: left) internal rotation of the $\mathrm{Sun}^{14}$, right) rotation of spiral galaxies ${ }^{15}$

Rotation frequencies of the Sun (from the core up) and rotational velocities of several spiral galaxies are shown on Fig. 7 .
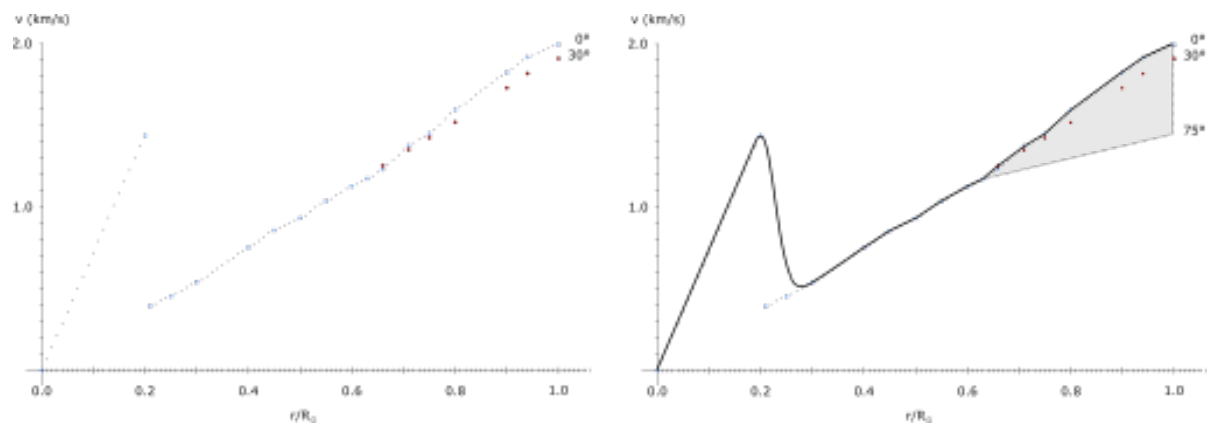

Figure 8: Rotational velocities of the Sun 
On the left, Fig. 8 shows the rotational velocities of the Sun based on rotation frequencies from two independent studies, one for the core $\left(\mathrm{r}<0.2 \mathrm{R}_{\odot}\right)$ and other from the core up (black dots are interpolated values, red dots show velocities at $30^{\circ}$ latitude).

On the right, Fig. 8 shows the complete velocity curve (with interpolated connection between two curves) and dispersion of velocities (shaded area) due to differential rotation in the convective zone.

Note that interpolated values do not represent the current state, rather initial state at the core when the discontinuity had more pronounced thickness. In the current state, discontinuity is extremely compressed and velocities increase sharply at $0.2 \mathrm{R} \odot$. This will be elaborated below.

What is obvious from the figures is that Sun rotates like a composition of two solid or rigid bodies (diverging only in the polar regions of the convective zone), consistent with condensation of $\mathrm{U}_{1}$ down and up quarks (energy levels) into two ground states $(+1 \mathrm{~s} /-1 \mathrm{~s})$.

Assuming the Sun is not solid anywhere (as expected in conventional theories), it should be mainly composed of plasma.

However, there is a possibility that fusion in stars operates differently (or at least has a secondary component) - through the bombardment of solid (or solid-like) material with particles produced in the radiative zone. These may be high energy photons produced through matter/antimatter annihilation and/or high temperature of plasma.

Evidently, velocity curve of the Sun is similar to a typical velocity curve of a spiral galaxy - in both cases there is an initial sharp increase in velocity in the core, followed by a decline, with each next increase in velocity being less steep than the previous one. Note that latitude dependent differential rotation may also be common at specific places in galaxies too.

If the spin momentum of the Sun is effectively immune to [large scale] collisions (even if the core would be solid, everything approaching the Sun is vaporized before reaching the surface), the only disturbance of Keplerian orbits must come from incomplete conversion of electro-magnetic potential and increase of temperature.

Assuming that orbital velocity is decreasing (from Keplerian velocity) proportionally to electro-magnetic potential, as hypothesized, orbital velocity of plasma should keep increasing with radius until it becomes equal to Keplerian velocity, beyond which point there should be no accumulation of charge and the radial component of the solar wind should dominate.

Using approximation of the velocity/radius dependence based on the velocity 
curve of the Sun (up to $130000 \mathrm{~km}$ from surface[16]), and equalizing with orbital law:

$$
v=\frac{2533.61175}{1.18686-0.1}\left(\frac{r}{R_{\odot}}-0.1\right)=\sqrt{\frac{G M_{\odot}}{r}}
$$

one obtains the orbit of such discontinuity:

$$
r=32.8 R_{\odot}=22.826 * 10^{6} \mathrm{~km} \approx 33 R_{\odot}
$$

First results from the Parker solar probe indicate a significant rotational velocity of the solar wind around $40 \mathrm{R}_{\odot}$, peaking at the closest approach. The results indeed indicate a high probability of a maximum velocity around $33 \mathrm{R}_{\odot}$ in case a rigid rotation of the solar wind is maintained up to that point.

Rigid rotation is a consequence of relative cancellation of neutral and electro-magnetic influence on angular velocity, making it dependent on real mass (solar wind) density (pressure) which for particle orbitals falls of proportionally to distance $r$ (number of particles per $2 \pi r$ is constant).

Note that, even without rigid rotation, the discontinuity should occur near the point where velocity becomes Keplerian, otherwise, higher velocity would indicate dark matter presence - another maximum.

Note that $33 \mathrm{R}_{\odot}$ is equal to $0.1 \mathrm{MAU}$ (Sun-Mars distance), while the above equation gives $0.1 \mathrm{R}_{\odot}$ for $\mathrm{v}=0$. This correlation of the radius of the Sun with the orbit of Mars is not a coincidence - Mars is the outermost positive charge of the $\mathrm{U}_{1} \cdot{ }^{10} \mathrm{C}$ atom (Solar System).

If the same is applied to the core of the Sun, the velocity at $0.2 \mathrm{R}_{\odot}$ should be equal to Keplerian velocity. Here, however, this velocity is the sum of Keplerian velocity of the surface maximum and a core maximum. For a surface maximum at $\mathrm{R}_{\odot}$ :

$$
\begin{array}{r}
v=s \sqrt{\frac{G M}{0.2 R_{\odot}}}+s \cdot \sqrt{\frac{G M_{\odot}}{R_{\odot}{ }^{2}} \frac{\left(0.2 R_{\odot}\right)^{2}}{R_{\odot}{ }^{2}} 0.2 R_{\odot}}=s \sqrt{\frac{G M}{0.2 R_{\odot}}}+s \cdot \sqrt{G M_{\odot} \frac{\left(0.2 R_{\odot}\right)^{3}}{R_{\odot}^{4}}} \\
\mathrm{~s}, \mathrm{~s} \odot \in\{-1,1\}
\end{array}
$$

where $\mathrm{M}$ is the mass of the core maximum, $\mathrm{s}$ is the spin polarization of gravity of the core maximum and $\mathrm{s}_{\odot}$ is the spin polarization of gravity of the surface maximum.

Equalizing this velocity with measured velocity at the core discontinuity:

$$
v=2 \pi * 0.2 R_{\odot} * f=2 \pi * 0.2 R_{\odot} * 1644 * 10^{-9}=1437.2545 \frac{\mathrm{m}}{\mathrm{s}}
$$


and setting spin polarization positive for counter-clockwise rotation [of the surface maximum], gives $s=-1$ and gravitational mass of the core roughly $3 / 2$ the Jupiter mass:

$$
M=2.951797 * 10^{27} \mathrm{~kg}
$$

which gives mean core density of:

$$
\rho=261.602486 \frac{\mathrm{kg}}{\mathrm{m}^{3}}
$$

implying the primary gravitational mass of the Sun is above the core. Difference in mass between the core and outer layers is roughly equal to the mass difference between inner and outer planets.

For the ratios to be equal, core mass must be 3 times higher, which indicates that space has been stretched (compressed, relative to core) from $0.286 \mathrm{R}_{\odot}(1.43$ * $0.2 \mathrm{R}_{\odot}$ ) to $0.2 \mathrm{R}_{\odot}$. Modifying the equation for Keplerian velocity accordingly would give the initial mass $\left(8.90211033 * 10^{27} \mathrm{~kg}\right)$ :

$$
\begin{aligned}
v & =s \sqrt{\frac{G M}{0.2 R_{\odot}} \frac{1.43}{1.43}}+s \odot \sqrt{G M_{\odot} \frac{\left(1.43 * 0.2 R_{\odot}\right)^{3}}{R_{\odot}^{4}}} \\
& =s \sqrt{\frac{G M}{0.2 R_{\odot}}}+s \odot \sqrt{G M_{\odot} \frac{\left(0.286 R_{\odot}\right)^{3}}{R_{\odot}^{4}}}
\end{aligned}
$$

Radius independent Keplerian velocities, like those at the outskirts of galaxies, are the effect of stretched space between maxima.

With shorter distance between maxima, minimum is more localized and changes in velocity are sharper.

Apparently, such stretching occurs in the Sun too.

Note that the equation S1.1 is defined by the straight line passing through $0.1 \mathrm{R}_{\odot}$ and $1.18686 \mathrm{R}_{\odot}$, so if one assumes that, without space stretching, the defining points would be $0.0 \mathrm{R}_{\odot}$ and $1.0 \mathrm{R}_{\odot}, 0.286 \mathrm{R}_{\odot}$ is the sum of translation of both points in radial direction due to stretching.

Note also that, if the Sun looses all outer mass with the collapse of the outer maximum, with leftover mass roughly equal to initial core mass, the Solar System becomes geocentric.

This stretching of space is evident on Fig. 9 in the sharp increase of velocity from $0.286 \mathrm{R}$ to $0.2 \mathrm{r}$. To conserve momentum, this increase in velocities in the inner half had to decrease velocities in the outer half of the Sun, up to 1.18686 $\mathrm{R}_{\odot}$.

Note that slower polar convective rotation could be the result of loss of shielding of the core maximum [charge] due to conversion of potential of the surface maximum (convergence from spherical to ring form). 


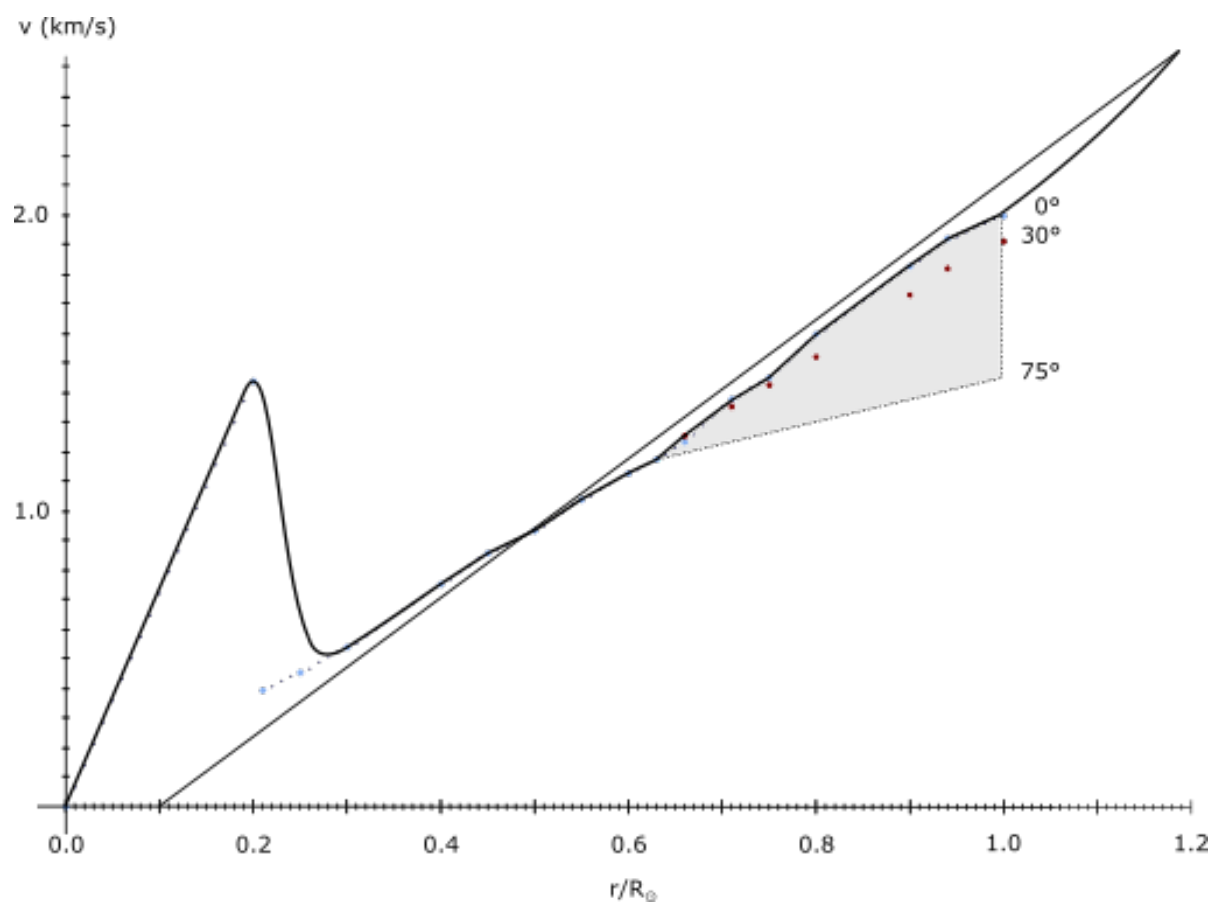

Figure 9: Rotational velocities of the Sun and near corona 
Gravity between the two maximums must be canceled near $0.2 \mathrm{R}_{\odot}$. Therefor, any particle escaping the core will overcome escape velocity at the surface of the Sun (if not slowed down by other particles). The same is true for the other direction. Thus, orbitals of particles at the discontinuity must be highly unstable and it should be the area of lowest [real mass] density.

However, gravitational stress can induce the collapse of the surface maximum. If that stress is low (insufficient for full collapse), the maximum will be fragmenting and collapsing into quanta of smaller charged maximum pairs, starting in polar regions (and, without further increase of stress, limited to polar regions).

At these places (sunspots), gravitational escape velocity is decreased allowing higher bandwidth of escaping mass, although significant transverse velocity component will exist, especially for charged particles.

Note that orbitals at polar regions seem to be entangled with the core. Strong entanglement between [quark] pairs may exist between the core and surface, it is also possible that gravitational stress is adding energy to such entanglement and inflating maxima of such pairs (creating wormholes).

In that case space is stretched from the cure to the surface (at sunspots) entangling orbital velocities but also being fixed to specific latitude by magnetic field lines (shielding inclined velocity component), the period of rotation of such plasma on the surface would be equal to:

$$
T=\frac{2 \pi R_{\odot}}{v}=3041363 s=35.2 \text { days }
$$

which is the rotation above $75^{\circ}$ latitude and should be the location of sunspot creation (inflation) at surface. Note that, once the orbital entanglement is lost, being charged, the sunspots will drift along the magnetic field lines.

The specific core discontinuity radius is the result of equilibrium between the outer gravitational force and induced vacuum force (forces cancel near the discontinuity).

The core gravitational maximum of the Sun might be the effect of vacuum, but, likely, all gravitational maxima are the result of vacuum induction and quantization.

In example, similar to inner Solar planets, the stars of a particular arm of a spiral galaxy could be the result of vacuum collapse into smaller quanta (maxima).

One might understand the creation of vacuum as stretching of space and 
decrease of density, but no space can be absolutely empty. Thus, if one is stretching space, one is also inflating smaller scale maxima.

The stretching of space between galaxies must result in creation of dark matter filaments between them. Intergalactic and galactic dark matter is thus likely created by dark energy, which is nothing else but a gravitational force of a large scale maximum well [of dark matter], expanding vacuum, just like it happens in the Sun.

Due to discretization of stable energy [levels], with enough energy applied into vacuum creation, the inflation will result in [relatively] permanent maxima of larger scale.

Vacuum inflation may be most likely in annihilation events, due to high symmetry and energy localization. However, stretching of space between strongly entangled particles can also result in permanently inflated particles (as in quark/anti-quark pairs).

If inflated particles are always of equal species to the original particles, evidently the [private] space of such particles is composed of the same particles but of lower scale.

In case of annihilation, the stretched (inflated) space might not be the space of annihilating pair, rather the underlying space, making the product of inflation highly dependent on the point of interaction. It may be more appropriate to state that, rather than being stretched in between, space is compressed at maxima. Similar to the 1st law, one could then construct another law:

\section{Space remains at constant density unless acted upon by grav-} itational force.

Thus, even if it may appear that, once deformed, no force is necessary to act on bodies in space to accelerate their radial motion relative to the sources of gravity, force (energy) is necessary to maintain such state of space.

As everything must conform to general oscillation, some force is always present, with relative magnitude and distance it is acting upon.

The speed of motion (radiation) will depend on density of space and, if gravitational force is limited, there will be a speed limit on motion. However, constancy of density is relative and even density is relative to the scale of the 1st order observer, or, more precisely, the strength of its entanglement with such space.

Absolute, and absolutely invariant limits are impossible. In any case, it seems that everything must be mirrored, and when it appears that is not the case, the cause is simply a large distance - in scale of space/time.

If the point of interaction of an annihilating pair imparts energy to the pair in highly asymmetric manner, the inflation would result in a pair of maxima of different scale (in fact, one of the particles could even be deflating). 
Thus, a possibility exists that even a proton and electron particles are the result of an anomaly in annihilation of particle/anti-particle pairs of equal scale.

Note that a gravitational maximum must have a radius - a point maximum would imply infinite gravity and no possibility for containment of smaller maxima.

Somewhere around the discontinuity, conditions may even be suitable for standard life. Note that the radius of the core is almost 22 times Earth radius, if density is not isotropic, smaller bodies might be orbiting inside.

Considering the momentum of the Solar System barycenter, density should not be isotropic.

\section{Quantization of momentum}

Previous works based on Titius-Bode law have shown that planetary orbits are quantized[17]:

$$
r=a e^{2 \lambda n}
$$

More recently it has been shown that distances and orbital periods are consistent with quantized scaling[18], rather than logarithmic.

If orbital radii are quantized, orbital (Keplerian) velocities are quantized.

Here, it will be shown that angular momentum is quantized too, as well as surface gravity.

If QM cannot describe the Solar System as an atom, it is QM that should be revised, not reality.

Orbital and spin angular momenta are correlated.

Note that spin radius cannot be 0 in CR. Every spin radius is thus orbital radius and if orbital radii are generally quantized, spin radii [and associated Keplerian velocities] should be too.

Gravitational maxima (event horizons) are, in an ideal case (no electromagnetic polarization), sphere surfaces with a well defined radius. Mass spin 
radius and velocity of a body (particle) are radius and velocity of its gravitational maximum.

Surface gravity of a planet depends on real mass content (defining surface radius) of the well and mass of the maximum. Assuming ratio of used capacity to full capacity for real mass between the planets is roughly the same and assuming ratio of mass of a gravitational maximum to [the square of] its radius is equal between particles on the same energy level, surface gravities of planets will be correlated.

If velocities and radii are quantized, and if momentum is quantized, gravitational mass must be quantized too. Gravitational mass is equal to the mass of a gravitational maximum.

If gravitational mass and radius of a maximum are quantized, its surface gravity must be quantized.

For outer planets, radius of the maximum is here hypothesized to be equal to what is currently defined as the surface radius (1 bar pressure level).

When quantized, orbital angular momentum satisfies the following equation:

$$
m v r=n \hbar
$$

where $\hbar$ is a constant, $\mathrm{n}$ is a positive integer number and $\mathrm{m}, \mathrm{v}, \mathrm{r}$ are components of orbital angular momentum - mass, velocity and radius, respectively.

Using total mass of the planet for $\mathrm{m}$ will not reveal quantization. In example, using Neptune's mass of $1.02413 * 10^{26} \mathrm{~kg}$ and setting $\mathrm{n}$ to 5 :

$$
m v r=5 \hbar=2.499714508 * 10^{42} \mathrm{Js}
$$

one obtains the scaled $\hbar$ (Planck's) constant for outer planets:

$$
\hbar=\hbar_{m_{2}}=4.999429016 * 10^{41} \approx 5 * 10^{41} \mathrm{Js}
$$

While the result is certainly interesting, the same $\hbar$ will not produce quantized momenta for other planets (it needs to be scaled).

The mass which should produce quantized angular momenta is, as previously established (equation Q1.4), real part of total mass.

However, if surface gravity is correlated with spin momentum, it must be correlated with orbital momentum too, and one may obtain the following equation for surface gravity:

$$
g=\frac{v r}{n \hbar} M_{N} g_{N}
$$

where $\hbar$ is equal to the obtained $\hbar$ above, $\mathrm{M}_{N}$ and $\mathrm{g}_{N}$ are Neptune's mass and surface gravity, respectively. In Table 3, required total mass is the total mass (gravitational energy) required to satisfy the quantization by standard QM (showing how far it can be from reality) based on obtained $\hbar$ relative to Neptune, calc. gravity is calculated surface gravity, while acc. is the surface acceleration taking rotation into account.

Protons and electrons are parts of two different universes (as difference in scale suggests), so one should use a different $\hbar$ constant for terrestrial planets (proton partons). 


\begin{tabular}{|c|c|c|c|c|c|c|c|c|}
\hline $\mathrm{n}$ & planet & $\begin{array}{l}\text { orbital } \\
\text { veloc- } \\
\text { ity v } \\
(\mathrm{m} / \mathrm{s})\end{array}$ & $\begin{array}{l}\text { orbital } \\
\text { radius } \\
\mathrm{r} \quad\left(10^{6}\right. \\
\mathrm{km})\end{array}$ & $\begin{array}{l}\text { total mass } \\
\mathrm{M} \quad\left(10^{24}\right. \\
\mathrm{kg})\end{array}$ & $\begin{array}{l}\text { required } \\
\text { total } \\
\text { mass } \\
\left(10^{24} \mathrm{~kg}\right)\end{array}$ & $\begin{array}{l}\text { calc. } \\
\text { grav- } \\
\text { ity g } \\
\left(\mathrm{m} / \mathrm{s}^{2}\right)\end{array}$ & $\begin{array}{l}\text { gravity } \\
\left(\mathrm{m} / \mathrm{s}^{2}\right)\end{array}$ & $\begin{array}{l}\text { acc. } \\
\left(\mathrm{m} / \mathrm{s}^{2}\right)\end{array}$ \\
\hline 5 & Neptune & 5430 & 4495.06 & 102.413 & 102.413 & 11.15 & 11.15 & 11.00 \\
\hline 5 & Uranus & 6800 & 2872.46 & 86.813 & 976 & 8.92 & 8.87 & 8.69 \\
\hline 3 & Saturn & 9680 & 1433.53 & 568.340 & 108.084 & 10.565 & 10.44 & 8.96 \\
\hline 1 & Jupiter & 13060 & 778.57 & 1898.190 & 49.168 & 23.225 & 24.79 & 23.12 \\
\hline
\end{tabular}

Table 3: Calculated gravity for outer planets

The angular momentum of Mercury $\left(\mathrm{m}=\mathrm{M}=3.3011 * 10^{23} \mathrm{~kg}\right)$ :

$$
m v r=5 \hbar=9.053654959 * 10^{38} \mathrm{Js}
$$

gives the scaled $\hbar$ constant for inner planets:

$$
\hbar=\hbar_{m_{1}}=1.810730992 * 10^{38} \mathrm{Js}
$$

Surface gravity for inner planets, using obtained $\hbar$, Mercury mass $\mathrm{M}_{M}$ and gravity $\mathrm{g}_{M}$ :

$$
g=\frac{v r}{n \hbar} M_{M} g_{M}
$$

In Table 4, showing calculated surface gravity for inner planets, required total

\begin{tabular}{l|l|l|l|l|l|l|l}
$\mathrm{n}$ & $\begin{array}{l}\text { planet (mir- } \\
\text { ror })\end{array}$ & $\begin{array}{l}\text { orbital } \\
\text { veloc- } \\
\text { ity } \\
(\mathrm{m} / \mathrm{s})\end{array}$ & $\begin{array}{l}\text { orbital } \\
\text { radius } \\
\mathrm{r}\left(10^{6}\right. \\
\mathrm{km})\end{array}$ & $\begin{array}{l}\text { total mass } \\
\left(10^{24} \mathrm{~kg}\right)\end{array}$ & $\begin{array}{l}\text { required } \\
\text { total } \\
\text { mass } \\
\left(10^{24} \mathrm{~kg}\right)\end{array}$ & $\begin{array}{l}\text { calc. } \\
\text { grav- } \\
\text { ity g } \\
\left(\mathrm{m} / \mathrm{s}^{2}\right)\end{array}$ & $\begin{array}{l}\text { gravity } \\
\left(\mathrm{m} / \mathrm{s}^{2}\right)\end{array}$ \\
\hline 5 & $\begin{array}{l}\text { Mercury } \\
\text { (Neptune) }\end{array}$ & 47360 & 57.91 & 0.330 & 0.33011 & 3.70 & 3.70 \\
3 & $\begin{array}{l}\text { Venus } \\
\text { (Uranus) }\end{array}$ & 35020 & 108.21 & 4.868 & 0.14335 & 8.52 & 8.87 \\
10 & $\begin{array}{l}\text { Earth (Sat- } \\
\text { urn) } \\
\text { Mars } \\
\text { Jupiter) }\end{array}$ & 29780 & 149.6 & 5.972 & 0.12193 & 10.02 & 9.798 \\
& 24070 & 227.92 & 0.642 & 0.33006 & 3.70 & 3.71
\end{tabular}

Table 4: Calculated gravity for inner planets

mass is the total mass based on $\hbar$ relative to Mercury, while the mirror is an outer planet candidate for [magnetic] spin entanglement.

Quantization can also be shown without using mass (directly), through the volumetric space-time momentum (gravitational momentum):

$$
g v r=n h\left[\frac{m^{3}}{s^{3}}\right]
$$

With h obtained from above, substituting mass with gravity, the equation for gravity becomes:

$$
g=\frac{v r}{n h} g_{0}^{2},
$$


where $g_{0}$ is the gravity of Neptune, or, in case of terrestrial planets, the gravity of Mercury, and it yields the same results.

While the second equation will yield the correct results for gravity, the equation gvr $=\mathrm{nh}$ will not, showing the inverse coupling of gravity to momentum:

$$
\frac{1}{g} v r=n h[m s]
$$

This gives, for outer planets:

$$
h=h_{g_{2}}=4.378148126 * 10^{14} \mathrm{~ms},
$$

for inner planets:

$$
h=h_{g_{1}}=1.482496 * 10^{14} \mathrm{~ms}
$$

Now, one can couple mass with gravity:

$$
\begin{gathered}
m v r=n \hbar_{m}, \frac{1}{g} v r=n h_{g}, \hbar_{m g}=\frac{\hbar_{m}}{h_{g}} \\
g=\frac{v r}{n h_{g}}=\frac{n \hbar_{m}}{m} \frac{1}{n h_{g}}=\frac{1}{m} \frac{\hbar_{m}}{h_{g}} \\
g=\frac{\hbar_{m g}}{m},
\end{gathered}
$$

and obtain relation to Sun's gravity:

$$
\begin{gathered}
r=\frac{n \hbar_{m}}{m v}=\frac{g n h_{g}}{v} \\
r^{2}=n^{2} \hbar_{m} h_{g} \frac{g}{m v^{2}} \\
\frac{m r}{g} \frac{4 \pi^{2} r^{3}}{T^{2}}=n^{2} \hbar_{m} h_{g}\left[k g m^{3}\right] \\
m r^{3} \frac{g_{S}}{g}=n^{2} \hbar_{m} h_{g}\left[k g m^{3}\right] \rightarrow v^{2}=r g_{S} \\
m^{2} r^{3} g_{S}=n^{2} \hbar_{m}{ }^{2}\left[\frac{k g^{2} m^{4}}{s^{2}}\right]
\end{gathered}
$$

where $g_{S}$ is the gravity of Sun at orbital radius $\mathrm{r}$.

For outer planets:

$$
\hbar_{m g}=\hbar_{m g_{2}}=1.14190495 * 10^{27} \frac{\mathrm{J}}{\mathrm{m}}=1.14190495 * 10^{27} \mathrm{~N}
$$

For inner planets:

$$
\hbar_{m g}=\hbar_{m g_{1}}=1.221407 * 10^{24} \mathrm{~N}
$$


The above obtained $\hbar_{m g}$ constants are based on total mass, for relative real mass, the quantum of gravitational force $\left(\hbar_{m g}\right)$ may be treated as invariant between inner and outer planets (with properly defined surface gravity g):

$$
\hbar_{m g}=6.968267285 * 10^{20} \mathrm{~N}
$$

Small deviation in gravity stems mainly from oscillation of surface gravity. Note, for example, with rotation taken into account $\left(\mathrm{g}_{N}=11.0\right.$ $\mathrm{m} / \mathrm{s}^{2}$ ) calculated gravity for Saturn would match exactly the measured value of 10.44 (which is the value without rotation!). On the other hand, the gravity of Jupiter with rotation closely matches the calculated value (without rotation). This confirms that the definition of surface relative to fixed pressure ( 1 bar in this case) is appropriate for outer planets but should oscillate (cycle) between planets to take into account fossilization of a previous maximum in rotation period.

Correlation with rotation is expected, as conversion between electromagnetic and gravitational potential affects both gravity and rotation of mass.

For terrestrial planets surface gravity is defined unrelated to pressure, as gravity at ground (sea) level. The calculated value matches Venus gravity at the transition zone between mesosphere and thermosphere.

For Earth, the value matches the transition zone between upper and lower mantle, or, if one calculates with constant mass, it is, just like in case of Venus, the value of height of the mesosphere/thermosphere transition zone, but negative (below surface). So, here too, the cyclic nature of surface gravity is evident.

The constants $\mathrm{h}(\hbar)$ and $\mathrm{G}$ (gravitational constant) are scale dependent, but they also must oscillate. The above results could thus be interpreted as due to oscillation of energy of space (as h/G directly depend on it). This oscillation may be, for the electron, confined to the atom, at least at non-condensing temperatures.

Another interpretation for the excitations of $\mathrm{G}$ is the absorption of large scale external gravitational waves, however, these cannot explain the confinement of the oscillation to atoms.

In any case, when comparing small scale atoms with large scale atoms (ie. planetary systems), one must not only choose a proper reference frame and take into account the possible effects of measurement, but resolve the issues of QM - make constants (properties of space) relative, with proper attribution of relativistic effects.

If surface gravity and spin radius are both quantized, then mass of the 
maximum must be quantized too:

$$
\begin{gathered}
g=\frac{G M}{r_{s}^{2}} \\
M=\frac{g}{G} r_{s}^{2}
\end{gathered}
$$

$\mathrm{g}=$ gravity of the maximum

$\mathrm{M}=$ mass of the maximum

$\mathrm{r}_{s}=$ radius of the maximum

and, with all three components quantized (m, v, r), the orbital angular momentum would now be quantized if mass would be the same for all inner/outer planets.

Indeed, looking at required total mass in Table 3 , the sole required mass that doesn't match others well is that of Jupiter. But that can easily be solved, if one assumes that energy level $\mathrm{n}$ is 2 instead of 1 .

It is similar for inner planets, setting $n=6$ for Venus and $n=9$ for Earth, yields good results. Note that, in both cases, $n$ is decreasing with a decrease in distance from the Sun.

However, masses between planets are not the same. But solution for that exists and it must be in vertical energy (mass) oscillation of particles between generations.

The fact that similar planets (Venus/Earth, Uranus/Neptune) share the energy level (n) fits well with the quantum hypothesis.

The relative high excitation of Mars $(n=10)$ and no excitation of Jupiter $(\mathrm{n}=1)$ indicates the system is in $6 \mathrm{p} 4 \mathrm{n}$ state.

Note that the following should be satisfied (with oscillations in superposition):

$$
\frac{N}{P} \frac{\hbar_{m_{2}}}{\hbar_{m_{1}}}=\left(1-\frac{h_{g_{1}}}{h_{g_{2}}}\right) \frac{\hbar_{m_{2}}}{\hbar_{m_{1}}}=\frac{m_{p}}{m_{e}},
$$

where $\mathrm{m}_{p}, \mathrm{~m}_{e}$ are masses of standard proton and electron, respectively. The factor $\mathrm{N} / \mathrm{P}$ is the ratio of neutrons to protons in the Solar System.

Some examples of planetary configurations for various states is shown in Table 5.

This shows direct entanglement of standard proton and electron mass with planetary mass and configuration.

As all constants, constant masses of standard protons and electrons are a superposition of oscillation. As with the $\hbar$ constant, the oscillation can be detected on large scale. 


\begin{tabular}{|c|c|c|c|}
\hline base & state & $\mathrm{N} / \mathrm{P}$ & surface gravity/orbital distance examples \\
\hline Carbon & $6 \mathrm{p} 4 \mathrm{n}$ & $4 / 6=2 / 3$ & $\begin{array}{l}\text { Mercury } 3.7(0.25 \text { MAU, } n=5) \text {, Venus } 8.87(0.5 \\
\text { MAU, n=3), Earth } 9.798(0.66 \text { MAU, } n=3) \text {, Mars } \\
3.71(1 \text { MAU, } n=10)\end{array}$ \\
\hline Boron & $5 \mathrm{p} 5 \mathrm{n}$ & $5 / 5=1$ & $\begin{array}{l}\text { Mercury B } 3.32(0.2 \text { MAU, } n=5) \text {, Venus/Earth A } \\
5.25(0.5 \text { MAU, } n=5) \text {, Earth B/Mars } 6.43(0.75 \\
\text { MAU, n=5) }\end{array}$ \\
\hline Beryllium & $4 \mathrm{p} 6 \mathrm{n}$ & $6 / 4=3 / 2$ & $\begin{array}{l}\text { Venus/Earth A } 1.85(0.25 \text { MAU orbit, } \mathrm{n}=10) \text {, } \\
\text { Earth B/Mars } 37.1(1 \mathrm{MAU}, \mathrm{n}=1)\end{array}$ \\
\hline
\end{tabular}

Table 5: Examples of discrete surface gravity and orbital distance for inner planets

On standard $\left(\mathrm{U}_{0}\right)$ scale, proton to electron mass ratio is:

$$
\frac{m_{p}}{m_{e}}=1836.15267343(11)
$$

On $\mathrm{U}_{1}$ scale:

$$
\begin{gathered}
\frac{N}{P} \frac{\hbar_{m_{2}}}{\hbar_{m_{1}}}=1840.66694172611441 \\
\left(1-\frac{h_{g_{1}}}{h_{g_{2}}}\right) \frac{\hbar_{m_{2}}}{\hbar_{m_{1}}}=1826.09096003909666
\end{gathered}
$$

From these, the value of superposition might be obtainable using the EH operator, ie. using 12/4 for the 1st order approximation:

$$
\begin{aligned}
& E H_{12 / 4}(, \lambda)+\left(1-\frac{h_{g_{1}}}{h_{g_{2}}}\right) \frac{\hbar_{m_{2}}}{\hbar_{m_{1}}}=\frac{m_{p}}{m_{e}}=1836.182024284 \\
& \lambda=\frac{N}{P} \frac{\hbar_{m_{2}}}{\hbar_{m_{1}}}-\left(1-\frac{h_{g_{1}}}{h_{g_{2}}}\right) \frac{\hbar_{m_{2}}}{\hbar_{m_{1}}}=\left(\frac{h_{g_{1}}}{h_{g_{2}}}+\frac{N}{P}-1\right) \frac{\hbar_{m_{2}}}{\hbar_{m_{1}}}
\end{aligned}
$$

The transition from $6 \mathrm{p} 4 \mathrm{n}$ to $5 \mathrm{p} 5 \mathrm{n}$ state likely includes:

- collapsing (vertical) scale of gravitational maxima,

- loss of one outer gravitational maximum (death of Neptune electron), dead matter remains,

- Mars' gravitational maximum fusing with one of Earth's gravitational maxima,

- fusion of Venus' gravitational maximum with remaining Earth's gravitational maximum, 
- Mercury loosing one gravitational maximum,

- small possibility of life changing base to boron,

- formation of a new dwarf planet in the main asteroid belt,

- space between planets expanding (Solar System expanding),

- Solar System increasing orbital momentum (velocity), decreasing spin momentum,

- spin momentum of planets increasing.

The transition from $5 \mathrm{p} 5 \mathrm{n}$ to $4 \mathrm{p} 6 \mathrm{n}$ state likely includes:

- scale collapse stop,

- loss of one outer gravitational maximum (Uranus e), dead matter remains,

- significant increase of Mars' gravity,

- death of Mercury, dead matter remains,

- significant increase of Venus' real mass, decreasing surface gravity,

- no complex surface life on Earth,

- formation of a new dwarf planet in the main asteroid belt,

- further expansion of space between planetary orbits,

- further increase of orbital momentum (velocity), decreasing spin momentum,

- further increase of planetary spins.

\subsection{Proper quantization in QM}

If one wants to compare the Solar System with a room temperature equivalent of a carbon atom in the context of QM, one must reduce the effects of exchange of em potential with neutral gravitational potential due to condensation and lepton oscillation.

In that case, real mass component of the total initial momentum (Q1.3, Q1.4), which is equal (relatively, but difference is negligible) between bound electrons, is the correct mass to be used in comparison.

Total initial momentum is the angular momentum, it is quantized and for all electrons in ground state should be equal to:

$$
m_{r e} v_{t o t} r_{a}=\frac{1}{2} \hbar
$$

However, generally, total momentum is the sum of orbital and spin components. 
Each quantum sub-shell may contain up to 2 electrons. If these are in condensed (bosonic) form, their momentums are strongly coupled, they will behave as a single body, and the proper equation for the magnitude of total angular momentum per sub-shell is:

$$
\begin{array}{r}
m_{r e} v_{t o t} r_{a}=\sqrt{l(l+1)} \hbar+s \hbar \\
v_{t o t}=v_{a}+v_{s}=v_{a}+\frac{2 \pi R_{s}}{T_{s}} \\
\mathrm{~T}_{s}=\text { spin rotation period }
\end{array}
$$

where $\mathrm{s}$ is the total [magnetic] spin of electrons in a sub-shell.

Generally, two fermionic particles have to have anti-aligned spin (ie. $-1 / 2$ and $+1 / 2$ ) to occupy the same sub-shell, however, with the exchange of electro-magnetic potential for gravitational potential the fermionic nature is converting to boson nature and some spin components can be annihilated. The annihilation of spin can be confined to single axis (in other directions, electro-magnetic magnitude is exchanged for gravitational), thus it is possible for a sub-shell to have 0 total spin even if it is occupied by a single particle (1e). It is also possible for total spin to be equal to 1 in 2 e states, but this may indicate that conversion started when 2 particles were separated (had the same magnetic spin).

Therefor, here spin momentum magnitude s can have the following values: $0, \pm 1, \pm 1 / 2$.

Since the value of $\mathrm{m}_{r e}$ here is constant, its value is irrelevant to prove QM equivalent quantization. For the sake of argument, let it be equal to $7^{*} 10^{19}$ $\mathrm{kg}$.

Since Jupiter has to be in 2e configuration (even if Solar System would not be the carbon [equivalent] atom), it is appropriate to derive $\hbar$ from its momentum.

Assuming $\mathrm{n}=1$ (as expected) for Jupiter, 1 must be equal to 0 , with s equal to 1 , the $\hbar$ is:

$$
\hbar=m_{r e} v_{t o t} r_{a}=1.382 * 10^{36} \mathrm{Js}
$$

Derived values of 1 and s (and obtained $\hbar$ using these values) for all the outer planets are shown in Table 6 . The obtained value of $\hbar$ for Uranus shows remarkable agreement with Neptune. The $\hbar$ values for Saturn and Jupiter still agree well with Neptune's $\hbar$ (up to the second decimal), but increase in value with increase in spin radius is obvious. Likely reason for this is oscillation of spin velocity (radius) as noticed previously in quantization of gravitational momentum. Note that this is equivalent to $\hbar$ oscillation, if one is to conserve discrete quantum numbers. 


\begin{tabular}{|c|c|c|c|c|c|c|c|c|c|c|}
\hline $\mathrm{n}$ & conf & 1 & $\mathrm{~s}$ & planet & $\begin{array}{l}\text { orbital } \\
\text { vel. } \\
\mathrm{v}_{a} \\
(\mathrm{~m} / \mathrm{s})\end{array}$ & $\begin{array}{l}\text { orbital } \\
\text { radius } \\
\mathrm{r}_{a} \\
\left(10^{6}\right. \\
\mathrm{km})\end{array}$ & $\begin{array}{l}\text { spin } \\
\text { vel. } \\
\mathrm{v}_{s} \\
(\mathrm{~m} / \mathrm{s})\end{array}$ & $\begin{array}{l}\text { spin } \\
\text { ra- } \\
\text { dius } \\
\mathrm{R}_{s} \\
(\mathrm{~km})\end{array}$ & $\begin{array}{l}\text { spin } \\
\text { rot. } \\
\text { pe- } \\
\text { riod } \\
\mathrm{T}_{s} \\
(\mathrm{~h}) \\
\end{array}$ & calc. $\hbar\left(J_{s}\right)$ \\
\hline 5 & $1 \mathrm{e}$ & 1 & $1 / 2$ & Neptune & 5430 & 4495.06 & 2668 & 24622 & 16.11 & $1.3310 * 10^{36}$ \\
\hline 5 & $1 \mathrm{e}$ & 1 & 0 & Uranus & 6800 & 2872.46 & 2568 & 25362 & 17.24 & $1.3319 * 10^{36}$ \\
\hline 3 & $2 \mathrm{e}$ & 1 & 0 & Saturn & 9680 & 1433.53 & 9538 & 58232 & 10.656 & $1.3636 * 10^{36}$ \\
\hline 1 & $2 \mathrm{e}$ & 0 & 1 & Jupiter & 13060 & 778.57 & 12293 & 69911 & 9.9250 & $1.3817 * 10^{36}$ \\
\hline
\end{tabular}

Table 6: Obtained values for $1, \mathrm{~s}$ and $\hbar$ for outer planets

However, the orbital radius oscillates too. Note that orbital velocity is almost equal to spin velocity for planets in 2e configuration (Jupiter and Saturn). Setting orbital velocity equal to equatorial spin velocity and decreasing spin velocity proportionally yields much better results for Jupiter:

$$
\hbar=m_{r e} r_{a} v_{t o t}=m_{r e} r_{a}\left(\frac{v_{e}}{v_{a}} v_{a}+\frac{v_{e}}{v_{a}} v_{s}\right)=1.33 * 10^{36} \mathrm{Js}
$$

$$
\mathrm{v}_{e}=12571 \mathrm{~m} / \mathrm{s}
$$

and, similarly, for Saturn:

$$
\begin{aligned}
\hbar=m_{r e} r_{a} v_{t o t} \frac{1}{\sqrt{2}}=m_{r e} r_{a}\left(\frac{v_{a}}{v_{e}} v_{a}+\frac{v_{a}}{v_{e}} v_{s}\right) \frac{1}{\sqrt{2}}=1.3372 * 10^{36} J s \\
\mathrm{v}_{e}=9871 \mathrm{~m} / \mathrm{s}
\end{aligned}
$$

These results show that constants in QM are the result of superposition of oscillating values.

One may attempt to do the same with positive charges (terrestrial planets), however, here, determination of spin radius is more challenging and spin rotation period is not primordial.

Instead of using matter velocity, better results should be obtainable using space (Keplerian) velocity at $\mathrm{R}_{s}$ (which is primordial):

$$
\begin{gathered}
\qquad v_{s}=\frac{2 \pi R_{s}}{T_{s}}=\sqrt{\frac{G M}{R_{s}}} \\
\mathrm{G}=\mathrm{G}_{0}=\text { standard gravitational constant }=6.674 * 10^{-11} \mathrm{~m}^{3} / \mathrm{kgs}^{2}
\end{gathered}
$$

One possible configuration is shown in Table 7 (with 1 and s of Earth/Mercury mirroring Saturn/Jupiter, Venus/Mars mirroring Uranus/Neptune, and spin velocity of Mercury set to its perihelion velocity). Note that roughly the same $\hbar$ 


\begin{tabular}{|c|c|c|c|c|c|c|c|c|c|c|}
\hline $\mathrm{n}$ & conf. & 1 & $\mathrm{~s}$ & planet & $\begin{array}{l}\text { total } \\
\text { mass } \\
\left(10^{24}\right. \\
\mathrm{kg})\end{array}$ & $\begin{array}{l}\text { orbital } \\
\text { vel. } \\
\mathrm{v}_{a} \\
(\mathrm{~m} / \mathrm{s})\end{array}$ & $\begin{array}{l}\text { orbital } \\
\text { ra- } \\
\text { dius } \\
\mathrm{r}_{a} \\
\left(10^{6}\right. \\
\mathrm{km}) \\
\end{array}$ & $\begin{array}{l}\text { spin } \\
\text { vel. } \\
\mathrm{v}_{s} \\
(\mathrm{~m} / \mathrm{s})\end{array}$ & $\begin{array}{l}\text { spin } \\
\text { radius } \\
\mathrm{R}_{s} \\
(\mathrm{~m})\end{array}$ & calc. $\hbar\left(\mathrm{Js}_{\mathrm{s}}\right)$ \\
\hline 10 & $1 \mathrm{e}$ & 1 & $1 / 2$ & Mars & 0.642 & 24070 & 227.92 & 27650 & 56044 & $4.3107 * 10^{35}$ \\
\hline 3 & $2 \mathrm{e}$ & 1 & 0 & Earth & 5.972 & 29780 & 149.6 & 28435 & 492971 & $4.3107 * 10^{35}$ \\
\hline 3 & $1 \mathrm{e}$ & 1 & 0 & Venus & 4.868 & 35020 & 108.21 & 45462 & 157195 & $4.3107 * 10^{35}$ \\
\hline 5 & $2 \mathrm{e}$ & 0 & 1 & Mercury & 0.330 & 47360 & 57.91 & 58980 & 6333 & $4.3107 * 10^{35}$ \\
\hline
\end{tabular}

Table 7: Possible configuration of inner planets

for Earth can be obtained by setting 1 to $1, \mathrm{~s}$ to $-1 / 2$, and spin velocity equal to Keplerian velocity at surface.

Note that spin radius $\mathrm{R}_{s}$ should correspond to a detectable discontinuity. By these results, this may be the inner inner core boundary or a dipole offset.

However, proper spin radius equivalent to the spin radius of outer planets can be calculated.

From Q1.2 - Q1.5 follows that current mass of a planet is a result of conservation of momentum (and velocity) during collapse of the orbital (non-localized) maximum to a spin maximum:

$$
m_{r e} v_{s} r_{a}=m_{i m g} v_{s} r_{s}
$$

With $\mathrm{m}_{\text {re }}$ equal to $7 * 10^{19} \mathrm{~kg}$ and with the assumption that $\mathrm{r}_{a}$ is, for all terrestrial planets, equal to current orbital radius, spin radius is:

$$
r_{s}=\frac{m_{r e}}{M} r_{a}
$$

Here, spin radius should correspond to charge radius. However, obtained radii

\begin{tabular}{l|c|c|l|l|r}
$\mathrm{n}$ & conf. & planet & $\begin{array}{l}\text { total mass } \\
\mathrm{M}\left(10^{24} \mathrm{~kg}\right)\end{array}$ & $\begin{array}{l}\text { orbital radius } \\
\mathrm{r}_{a}\left(10^{6} \mathrm{~km}\right)\end{array}$ & spin radius $\mathrm{r}_{s}(\mathrm{~m})$ \\
\hline 10 & $1 \mathrm{e}$ & Mars & 0.642 & 227.92 & 24851090 \\
3 & $2 \mathrm{e}$ & Earth & 5.972 & 149.6 & 1753428 \\
3 & $1 \mathrm{e}$ & Venus & 4.868 & 108.21 & 1556019 \\
5 & $2 \mathrm{e}$ & Mercury & 0.330 & 57.91 & 12283939
\end{tabular}

Table 8: Calculated spin radius for inner planets

for Mercury and Mars are much larger then their current surface radii, indicating that either the collapse did not occur at $\mathrm{r}_{a}$ or there were additional collapses. 
Interestingly, calculated spin radius of Mars is roughly equal to radius of Neptune. It is also roughly equal to orbital radius of Deimos, the outermost moon of Mars, which may be interpreted as evidence of Mars' primordial (ground state) charge radius and a source of quantization of Moon radii.

If that is indeed the case, remains of moon charges of Mercury might also be present around the $12 \mathrm{k} \mathrm{km}$ orbit and small deviation between the obtained spin radius and the orbit of Deimos may be attributed to oscillation of radii or mass (real mass of $6.6 * 10^{19} \mathrm{~kg}$ gives the orbit of Deimos).

I believe current moons of Mars are remains of larger moons the gravitational maxima of which have collapsed into Mars in the process of planetary neurogenesis (hypothesis which will be presented later), thus, it is possible the original orbit was equal to obtained spin radius.

Collapse of moons in this process is simultaneous with the recession of the magnetic field. Moons with a distinct gravitational maximum are thus entangled with the magnetic dipole of the planet. Considering orbital period of Deimos is $30 \mathrm{~h}$, only a couple of hours larger than the rotation period of Mars, the original Moon might have even had a direct role in the production of Mars' magnetic field.

If the obtained spin radius is the ground state radius, the excited radius [for terrestrial planets] is likely the ground radius divided by $\mathrm{n}$.

For Mercury and Mars, this gives roughly the radius of Mercury $(2 \mathrm{x}$ radius of Mars' core, 2x radius of Earth/Mercury inner core).

For Earth, this gives the inner inner core radius or possibly dipole offset maximum (the dipole offset orbital [radius] thus being the real charge radius, opposed to the induced one in the outer core).

Note that, core differentiation into molten outer and solid inner part should be associated with 2e configuration. Both Mercury and Earth are hypothesized to be in 2e configuration and both presently do have differentiated cores. Current data on Mars indicates its core is likely entirely liquid, again, consistent with hypothesized 1e configuration. The same should thus be true for Venus.

However, even in 1e configuration, core splitting is expected in the early stage of planet development and may even periodically occur in adult form.

If Solar System maxima are oscillating between ${ }^{10} \mathrm{C}$ and ${ }^{10} \mathrm{Be}$ configuration, even with a change in scale [of a maximum], Mars must periodically exist in 2e configuration (acquiring one of Earth's maxima, while Earth acquires Venus' maximum).

Even if lifetime of a ${ }^{10} \mathrm{Be}$ configuration may be short, created temperature difference in the core should be sustainable over longer periods of time if the collapse of $2 \mathrm{e}$ configuration induces splitting of a 1e maximum into 2 quanta. 
Since both Mars and Venus appear to have been habitable on surface some time ago, both must have been in such configuration. The switch likely occurs with the end of a 1st order Solar System cycle (4.25 Gy), but it likely also has precursors of shorter duration with the end of 2nd $(\approx 26 \mathrm{My})$ and $3 \mathrm{rd}(1.512 \mathrm{My})$ order cycles.

There is a high possibility that Mars' surface becomes habitable for a short period of time with the end of each cycle, not only when these are synchronized with the end of a major (1st order) cycle.

In any case, as I am convinced the Solar System is at the end of a major cycle, I believe the magnetic field of Mars will be restored within a decade or a couple of decades at most, and, once it is stabilized, should persist for millions of years.

If $1 \mathrm{e}$ configuration can split into a $2 \mathrm{e}$ equivalent, two maxima of $2 \mathrm{e}$ configuration can certainly fuse into a 1e equivalent maximum. Generally, this happens when a planet reaches the adult stage (acquired real mass with its own gravity stimulates inflation of the inner core maximum), but the same effect can also be achieved with suspended animation (spin momentum), as demonstrated by Mercury.

Simultaneously with increasing habitability of Mars, one can thus expect decreasing habitability of Earth.

\subsection{Quantization of radii and gravity}

From:

$$
\frac{1}{g} v r=n h_{g}
$$

and:

$$
v^{2}=r g_{s}=r \frac{G M_{\odot}}{r^{2}}=\frac{G M_{\odot}}{r}
$$

follows:

$$
r=n^{2} \frac{g^{2} h_{g}{ }^{2}}{G M_{\odot}}=n^{2} \frac{G M^{2} h_{g}{ }^{2}}{r_{s}{ }^{4} M_{\odot}}
$$

While, from Q2.1 and Q2.2, orbital radius is:

$$
\begin{gathered}
r=\frac{1}{m_{r e} v_{t o t}}(\sqrt{l(l+1)}+s) \hbar=\frac{1}{m_{r e}\left(\sqrt{\frac{G M_{\odot}}{r}}+\sqrt{\frac{G k M_{\odot}}{r}}\right)}(\sqrt{l(l+1)}+s) \hbar \\
r=\frac{1}{m_{r e}{ }^{2} G M_{\odot}(1+\sqrt{k})^{2}}(\sqrt{l(l+1)}+s)^{2} \hbar^{2}
\end{gathered}
$$

For outer planets:

$$
\hbar=g_{0} h_{g} m_{r e}=1.3310 * 10^{36} \mathrm{Js}
$$


Here, square root of $\mathrm{k}$ is another quantum momentum magnitude [sum], shown in Table 9. From Q2.3 and Q2.4 follows that surface gravity is quantized:

\begin{tabular}{l|c|c|c|c|r}
$\mathrm{n}$ & configuration & $\mathrm{l}$ & $\mathrm{s}$ & planet & $\sqrt{k}$ \\
\hline 5 & $1 \mathrm{e}$ & 1 & $1 / 2$ & Neptune & $\frac{1}{2}$ \\
5 & $1 \mathrm{e}$ & 1 & 0 & Uranus & $\sqrt{\frac{1}{2}\left(\frac{1}{2}+1\right)}-\frac{1}{2}=\frac{\sqrt{3}}{2}-\frac{1}{2}$ \\
3 & $2 \mathrm{e}$ & 1 & 0 & Saturn & 1 \\
1 & $2 \mathrm{e}$ & 0 & 1 & Jupiter & $\sqrt{\frac{1}{2}\left(\frac{1}{2}+1\right)}=\frac{\sqrt{3}}{2}$
\end{tabular}

Table 9: Obtained k momentum for outer planets

$$
g=\frac{1}{n} \frac{(\sqrt{l(l+1)}+s)}{(1+\sqrt{k})} g_{0}
$$

where $\mathrm{g}_{0}$, equal to $43.43 \mathrm{~m} / \mathrm{s}^{2}$, is the quantum of gravity.

From Q2.3 and with total mass equal to:

$$
M=m_{i m g}=m_{r e} \frac{r_{a}}{r_{s}}=m_{r e} \frac{r}{r_{s}}
$$

follows that spin radius $\mathrm{r}_{s}$ is quantized too:

$$
r_{s}{ }^{6}=n^{2} r \frac{G m_{r e}{ }^{2} h_{g}{ }^{2}}{M_{\odot}}
$$

Combined with Q2.4 and Q2.5:

$$
\begin{gathered}
r_{s}{ }^{3}=n \frac{(\sqrt{l(l+1)}+s)}{(1+\sqrt{k})} \frac{\hbar^{2}}{g_{0} m_{r e} M_{\odot}} \\
r_{s}=\left[n \frac{(\sqrt{l(l+1)}+s)}{(1+\sqrt{k})} \frac{g_{0} m_{r e} h_{g}{ }^{2}}{M_{\odot}}\right]^{\frac{1}{3}}
\end{gathered}
$$

Note that the constant on the right is, for $\mathrm{m}_{r e}=7 * 10^{19} \mathrm{~kg}$, equal to:

$$
\frac{g_{0} m_{r e} h_{g}{ }^{2}}{M_{\odot}}=2.93050621 * 10^{20} \mathrm{~m}^{3}
$$

apparently an integer multiple of the speed of light on $\mathrm{U}_{1}$ scale $(2.93$ * $\left.10^{6} \mathrm{~m} / \mathrm{s}\right)$. 
For Neptune this gives spin (charge) radius equal to half of the current surface radius - as expected, like in case of Earth, real charge radius should be half of the mass radius of the maximum (for Earth, mass radius of the maximum is the inner core radius).

Note that dipole offset for Neptune is roughly half the radius, consistent with the result.

For $\mathrm{m}_{r e}$ equal to $5.6 * 10^{20} \mathrm{~kg}$, one gets the mass radius of the maximum (surface radius).

The result is similar for Uranus.

Note that the equation might not give accurate current spin radius for Jupiter and Saturn. Reason for this may be that the initial assumption of current radius being equal to collapse radius $\left(\mathrm{r}_{a}=\mathrm{r}\right)$ is not valid, however, more likely explanation is oscillation of mass (and therefor, spin radius) - even if the Solar System is carbon-like, its negative and positive charge components are not necessarily all electrons and positrons.

Inflation of mass can be asymmetric due to lepton oscillation.

However, the result for Jupiter gives radius exactly two times the dipole offset of Saturn in surface radius relative units $(2 \times 0.03778 \mathrm{R}=0.07557$ $\mathrm{R})$, but roughly $2 / 3$ the actual dipole offset of Jupiter $(0.119 \mathrm{R})$. The value is also equal to dipole offset of Earth $(0.076 \mathrm{R})$.

On the other hand, the result for Saturn gives radius $0.146 \mathrm{R}(4 \times 0.0365$ $\mathrm{R}$ ), closer to dipole offset of Jupiter.

Again, these results suggest the cause for discrepancy is oscillation.

For inner planets, the constants are different:

$$
\begin{gathered}
h_{g}=h_{g_{1}}=1.482496 * 10^{14} \mathrm{~ms} \\
\hbar=g_{0} h_{g} m_{r e}=4.5069360896 * 10^{35} \mathrm{Js}
\end{gathered}
$$

and possible quantization parameters, along with the calculated spin radius, are shown in Table 10. Note that the above parameters for Mars' orbital radius give

\begin{tabular}{l|c|c|c|c|c|r}
$\mathrm{n}$ & conf. & $\mathrm{l}$ & $\mathrm{s}$ & planet & $\sqrt{k}$ & spin radius $(\mathrm{m})$ \\
\hline 10 & $1 \mathrm{e}$ & 1 & $1 / 2$ & Mars & $\sqrt{\frac{1}{2}\left(\frac{1}{2}+1\right)}+\frac{1}{2}=\frac{\sqrt{3}}{2}+\frac{1}{2}$ & 6477988 \\
3 & $2 \mathrm{e}$ & 1 & 0 & Earth & 1 & 4146215 \\
3 & $1 \mathrm{e}$ & 1 & 0 & Venus & $\sqrt{\frac{1}{2}\left(\frac{1}{2}+1\right)}+\frac{1}{2}=\frac{\sqrt{3}}{2}+\frac{1}{2}$ & 3920325 \\
5 & $2 \mathrm{e}$ & 0 & 1 & Mercury & $\sqrt{\frac{1}{2}\left(\frac{1}{2}+1\right)}+\frac{1}{2}=\frac{\sqrt{3}}{2}+\frac{1}{2}$ & 4140950
\end{tabular}

Table 10: Possible quantization parameters and spin radii for inner planets

a perihelion rather than a semi-major axis, suggesting that it (and generally, planets with large eccentricity) may be in a superposition of two quantum states. 
Results for spin radius are obviously wrong, most likely reason for this is the bad $\mathrm{h}_{g}$ constant as it is based on gravity at surface radius, which, for inner planets, is not defined as the radius of a gravitational maximum.

However, correlation with dipole offsets is still present. Calculated spin radius of Earth/Mercury is almost exactly 10 times the experimentally obtained dipole offset of Mercury (414.7 km).

If the assumption of charge radius being 10 times lower than calculated spin radius for terrestrial planets is valid, somewhat larger current offset for Earth (484.7 km from center) must be the result of oscillation (superposition) and faster rotation.

Consistent correlation of results with dipole offsets suggests the primary or primordial source of magnetic dipoles in planets is concentrated (collapsed) orbiting charge with a large spin momentum close to the dipole offset radius, rather than currents induced with Coriolis force in outer parts of differentiated cores.

In fact, deviation of a dipole offset from calculated value should, in some part, be due to induced currents rather than oscillation.

In that case, faster rotation rates and greater liquid mass would introduce greater deviation. This is consistent with obtained results, as Jupiter and Saturn do rotate much faster than Uranus and Neptune, while Earth rotates much faster than Mercury.

However, as calculated and experimentally obtained dipole offsets both seem to be multiples of $\approx 0.034-0.038 \mathrm{R}$, only deviation from integer multiples of that quantum may be attributed to induced currents, the rest is more likely due to [quantized] oscillation.

Using the radius of a gravitational maximum for Earth $(1206115 \mathrm{~m})$, one obtains the proper $\mathrm{h}_{g}$ constant for charge radius calculation of inner planets:

$$
\begin{array}{r}
h_{g}=h_{g_{1}}=\frac{v r}{g n}=5.419815085 * 10^{12} \mathrm{~ms} \\
\mathrm{v}=\text { Earth's orbital velocity }=29780 \mathrm{~m} / \mathrm{s} \\
\mathrm{r}=\text { Earth's orbital radius }=149.6^{*} 10^{9} \mathrm{~m} \\
\mathrm{~g}=\text { gravity of the maximum }=274 \mathrm{~m} / \mathrm{s}^{2} \\
\mathrm{n}=3
\end{array}
$$

Results obtained using this constant are shown in Table 11. These are now much closer to dipole offsets. Difference should be attributed to oscillation.

Models of the dipole location of Earth indeed show oscillation, in the last 10000 years it has oscillated from a maximum of $414.7 \mathrm{~km}$ (equal to a dipole offset of Mercury) in the western hemisphere to a maximum of $554.7 \mathrm{~km}$ in the eastern hemisphere[19].

Dipole offset in current models is thus a superposition (arithmetic mean) of these two maxima $(484.7 \mathrm{~km})$. 


\begin{tabular}{l|c|c|c|c|c|c}
$\mathrm{n}$ & conf. & $\mathrm{l}$ & $\mathrm{s}$ & planet & $\sqrt{k}$ & spin radius $(\mathrm{m})$ \\
\hline 10 & $1 \mathrm{e}$ & 1 & $1 / 2$ & Mars & $\sqrt{\frac{1}{2}\left(\frac{1}{2}+1\right)}+\frac{1}{2}=\frac{\sqrt{3}}{2}+\frac{1}{2}$ & 1 \\
3 & $2 \mathrm{e}$ & 1 & 0 & Earth & 413566 \\
3 & $1 \mathrm{e}$ & 1 & 0 & Venus & $\sqrt{\frac{1}{2}\left(\frac{1}{2}+1\right)}+\frac{1}{2}=\frac{\sqrt{3}}{2}+\frac{1}{2}$ & 436716 \\
5 & $2 \mathrm{e}$ & 0 & 1 & Mercury & $\sqrt{\frac{1}{2}\left(\frac{1}{2}+1\right)}+\frac{1}{2}=\frac{\sqrt{3}}{2}+\frac{1}{2}$ & 456136
\end{tabular}

Table 11: Possible quantization parameters and spin radii for inner planets, with corrected $\mathrm{h}_{g}$

The agreement of $414.7 \mathrm{~km}$ maximum with the dipole offset of Mercury suggests that either:

- the influence of rotation on the offset is negligible,

- rotation stops once the maximum is reached,

- induced currents are created at the expense of primary charge, effectively transferring the charge radius from inner core to outer core.

Possibly, this is the effect of conservation of momentum, where spin of the primary charge is reduced at the expense of core rotation.

\subsection{Lepton oscillation model}

In the previous chapter it was hypothesized that the discrepancy between the QM model of the atom and the Solar System can be resolved by lepton oscillation.

This can be solely mass oscillation, which requires external energy, or the oscillation of general force flavor which does not require external energy as mass is inflated with the exchange of polarized (electro-magnetic) potential with a neutral gravitational potential (it does need stimulation though, most likely by resonance - synchronization).

However, while general force flavor has certainly been changed to [dominantly] neutral with a change in vertical energy level, difference in mass between the outer planets is too large compared to a difference in electro-magnetic energy to be explained by general force oscillation alone.

If the Solar System has been inflated, as hypothesized, from a smaller scale atom, then likely there was enough energy for a superposition of electron mass eigenstates.

Taking into account that these electrons are also neutralized, superposition becomes even more likely (charged leptons repel) due to lower energy requirements. 
With oscillation and inflation taken into account, the fact that planets of the Solar System have different masses goes in favor of it being the atom, rather than against it.

However, the excess energy left after the vertical energy level increase (inflation) might not be the only source of superposition. Most energy in the vertical energy level change is spent on inflation - not flavor oscillation, so even without inflation, the flavor oscillation energy can be provided by the nucleus or absorption of properly scaled gravitational waves.

Atoms which are not under influence of strong external magnetic fields may be dominantly in neutral or oscillating configuration, regardless of scale - there is a lot of energy for mass oscillation in nucleons.

If neutrinos oscillate in flight they must be absorbing energy in space, but their flavor may instead be predetermined with oscillation of particles inside the atom. In fact, lepton oscillation [of electron scale particles] might be confined to atoms if discharge of outermost particles is synchronized with their mass flavor being in ground (lowest energy) state in a form of electron.

There is no such requirement for neutrinos as their mass is much lower than that of the electron. Probability of discharge of masses greater than electron mass might be simply too low due to much greater gravitational attraction.

With no absolute constants allowed and implied oscillation of relative constants, oscillation in the energy of space is predicted by CR.

With no oscillation, in the Solar System, the inner planets would all be in positron equivalent states, while outer planets would be scaled electrons.

Note that, with em force almost completely neutralized (especially for inner planets), due to equal energy of positrons and electrons there are no large differences between these particles, apart from anti-alignment of magnetic spins.

One might ask why and how are positrons created (extracted) here? The answer is in neutralization - when charged they balance the electrons and, most likely, they are, together with neutrinos (main dwarf planets), the result of $\beta^{+}$decay of protons. However, due to charge neutralization, instead of being ejected from the nucleus, they remain bound to it.

The $\beta^{+}$process implies that each positron $\left(1 \mathrm{e}^{+}\right.$terrestrial planet) or positron pair $\left(2 \mathrm{e}^{+}\right.$terrestrial planet) is entangled with a specific neutrino 
( $1 \mathrm{v}_{e}$ main dwarf planet) or a neutrino pair ( $2 \mathrm{v}_{e}$ main dwarf planet) since the entangled pairs have been created at the same time, through the intermediate $\mathrm{W}^{+}$boson.

Note also, that, in order for the equation Q1.1 to remain valid, the excitation of Neptune must be equal to the [scaled] excitation of the nucleus (Sun).

Thus, for the Solar System atom, and perhaps generally, the oscillation is synchronized between the innermost and outermost parts of the atom, consistent with absorption of wave-like forms of energy.

The oscillation can thus explain the difference in masses between the planets, but the oscillation itself should be quantized.

\subsubsection{The creation}

Applying neutralization and lepton oscillation to the model of inflation (vertical energy level change), one can now reconstruct the history of the Solar System development.

With inflation, the [absolute] distance between particles is increasing. Assuming the system started in polarized state, neutralization will be decreasing [relative] distance between equally charged particles.

Note 1: The only reason for neutralization during inflation may be a difference in [relative] external magnetic field strengths between the two scales, with larger scale system being under influence of much weaker magnetic fields.

Note 2: If the inflation starts with an already neutral system, the end result is similar. In that case, large scale system is a relative clone of the small scale system, with no energy wasted on neutralization, only on inflation. However, any excess energy (beyond the discrete energy needed to change the vertical energy level) will result in cloning imperfections proportional to the excess energy.

Note 3: Only naked [gravitational] maxima are inflated, real mass is acquired during and after inflation from existing mass (asteroid) fields. These fields are generally created with deflation of other maxima in nova like explosions. Deaths (deflations) and births (inflations) of a particular scale are relatively synchronized.

Based on wave-like appliance of energy, the inflation may have proceeded in this order: 
1. Nucleus started inflating.

2. Jupiter 2e configuration started inflating. Even though 2e may have been separated initially, large energy of this configuration enabled the fusion of 2 electrons. With the inflation of Jupiter, 2e positron configuration was inflating. However, this configuration did not have enough energy for fusion and the positrons were left separated enough to form Mars $\left(1 \mathrm{e}^{+}\right)$ and Vulcan $\left(1 \mathrm{e}^{+}\right.$, Mercury embryo).

3. Saturn 2e configuration started inflating. This one had less energy that Jupiter 2e, but still enough for fusion, while the positrons again, did not - however, the energy was bigger than in the first positron pair, resulting in the creation of Venus $\left(1 \mathrm{e}^{+}\right)$and Gaia $\left(1 \mathrm{e}^{+}\right.$, Earth embryo).

4. Another 2e configuration started inflating. This one had even less energy than Saturn 2e, and, this time, not enough for fusion, so 2e separated into Uranus (1e) and Neptune (1e). A [relatively] simultaneous $2 \mathrm{e}^{+}$inflation resulted in fusion of $1 \mathrm{e}^{+}$with Vulcan, creating Mercury, and fusion of the other $1 \mathrm{e}^{+}$with Gaia, creating Earth.

Note that, on the right (outer) side, the energy of inflation is decreasing, while on the left (inner) it is increasing. This fluctuation is the result of an attempt to balance the left and right side of the system.

Note also, that, if the original (small scale) system was in an electric field, the system did have a left and right (or top and bottom) side, not only inner and outer orbits.

Comparing energies of planets, lepton oscillation and the [attempt of] energy balancing is obvious.

Assuming that scaled mass of a standard electron $\left(0.511 \mathrm{MeV} / \mathrm{c}^{2}\right)$ is equal to $0.511 * 10^{24} \mathrm{~kg}$, scaled muon $\left(105.658 \mathrm{MeV} / \mathrm{c}^{2}\right)$ is $105.658 * 10^{24} \mathrm{~kg}$, while scaled tau particle $\left(1776.86 \mathrm{MeV} / \mathrm{c}^{2}\right)$ has a mass of $1776.86 * 10^{24} \mathrm{~kg}$, rough correlation with masses of Mercury/Mars, Neptune/Uranus and Jupiter is obvious.

The tau/muon/electron mass ratios are present within the inner and outer planets:

$$
\begin{gathered}
\frac{\text { Venus }+ \text { Earth }}{\text { Mars }} \approx \frac{\text { Venus }+ \text { Earth }}{2 \text { Mercury }} \approx \frac{\text { tau }}{\text { muon }} \\
\frac{\text { Neptune }}{\text { Earth }} \approx \frac{\text { Uranus }}{\text { Venus }} \approx \frac{\text { Jupiter }}{\text { Neptune }} \approx \frac{\text { tau }}{\text { muon }} \\
\frac{\text { Outer planets }}{\text { Inner planets }} \approx \frac{\text { Uranus }+ \text { Neptune }}{\text { Mercury embryo }+ \text { Mars }} \approx \frac{\text { muon }}{\text { electron }}
\end{gathered}
$$

but also in relation to the Sun:

$$
\frac{\text { Sun }}{\text { Saturn }} \approx \frac{\text { tau }}{\text { electron }}
$$


which suggests that the whole system is in superposition of particles of different generations.

The grouping and correlation of Venus/Earth and Uranus/Neptune here is understandable, as the pairs share the same quantum shell.

Correlation of Uranus/Neptune with Mercury/Mars lies in the fact that Mars and Mercury [embryo] were the first pair created on the inner side, while Uranus and Neptune were the last to be created on the outer side with increasing energy on the inner side and decreasing on the outer side, the ratio of Uranus +Neptune/Mercury+Mars becomes roughly equal to the ratio of mass of outer to inner planets. This gives mass of 0.198 * $10^{24} \mathrm{~kg}$ for the Mercury embryo $\left(1 \mathrm{e}^{+}\right)$. Comparing Venus $\left(1 \mathrm{e}^{+}\right)$and Earth $\left(2 \mathrm{e}^{+}\right)$, the addition of another maximum doesn't impact the total mass significantly (as most energy comes from neutralization which is, at least roughly, invariant to number of particles occupying the state).

If the Mercury embryo mass was core mass, total core mass of current Mercury should be equal to:

$\frac{\text { Earth core }}{\text { Venus core }}$ (Mercury embryo mass) $=\frac{0.325}{0.32} 0.198 * 10^{24} \mathrm{~kg}=0.2011 * 10^{24} \mathrm{~kg}$

, $61 \%$ of its total mass (for Venus' core at $32 \%$ of total mass, Earth's core at $32.5 \%$ of total mass).

In Table 12, standard particle candidates are shown for each planet. Rest masses are relative to the possible event horizon of creation, specified in parentheses. Note that original rest mass may be bigger or smaller than relativistic mass, depending on the conditions in the annihilation (creation) event.

Most likely particle candidates are marked green. Rest mass in Table 12 was

\begin{tabular}{|c|c|c|c|}
\hline planet & $\begin{array}{l}\text { relativistic mass } M \\
{\left[10^{24} \mathrm{~kg}\right](\mathrm{v})}\end{array}$ & $\begin{array}{l}\text { rest mass } \mathrm{M}_{0} \text { candidates }\left[\begin{array}{ll}10^{24} & \mathrm{~kg}\end{array}\right] \\
\left(\mathrm{c}_{E H}\right)\end{array}$ & particle candidates $\left(\mathrm{MeV} / \mathrm{c}^{2}\right)$ \\
\hline Mercury & $0.330(47.4 \mathrm{~km} / \mathrm{s})$ & $\begin{array}{l}0.361(19.34 \mathrm{~km} / \mathrm{s}=\text { Vesta orbit }) \\
0.353(16.76 \mathrm{~km} / \mathrm{s}=\text { Hygiea orbit }) \\
0.383(24.1 \mathrm{~km} / \mathrm{s}=\text { Mars orbit }) \\
0.489(35 \mathrm{~km} / \mathrm{s}=\text { Venus orbit })\end{array}$ & $?(0.198)$, positron $(0.511)$ \\
\hline Venus & $4.868(35.0 \mathrm{~km} / \mathrm{s})$ & $\begin{array}{l}5.67(17.905 \mathrm{~km} / \mathrm{s}=\text { Ceres orbit }) \\
5.545(16.76 \mathrm{~km} / \mathrm{s}=\text { Hygiea orbit })\end{array}$ & down anti-quark $(\approx 4.8)$ \\
\hline Earth & $5.972(29.8 \mathrm{~km} / \mathrm{s})$ & $\begin{array}{l}7.47(17.905 \mathrm{~km} / \mathrm{s}=\text { Ceres orbit }), \\
7.47(17.89 \mathrm{~km} / \mathrm{s}=\text { Pallas orbit }) \\
4.77(-17.905 \mathrm{~km} / \mathrm{s}=- \text { Ceres orbit })\end{array}$ & down anti-quark $(\approx 4.8)$ \\
\hline Mars & $0.642(24.1 \mathrm{~km} / \mathrm{s})$ & $\begin{array}{l}1.076(19.34 \mathrm{~km} / \mathrm{s}=\text { Vesta orbit), } \\
0.383(-19.34 \mathrm{~km} / \mathrm{s}=- \text { Vesta orbit }), \\
0.461(-16.76 \mathrm{~km} / \mathrm{s}=- \text { Hygiea orbit }), \\
0.539(-13.1 \mathrm{~km} / \mathrm{s}=- \text { Jupiter orbit })\end{array}$ & positron $(0.511)$ \\
\hline Jupiter & $1898.19(13.1 \mathrm{~km} / \mathrm{s})$ & $\begin{array}{l}1396(-19.34 \mathrm{~km} / \mathrm{s}=- \text { Vesta orbit }) \\
1293(-17.905 \mathrm{~km} / \mathrm{s}=- \text { Ceres orbit }) \\
1824(-47.4 \mathrm{~km} / \mathrm{s}=- \text { Mercury orbit })\end{array}$ & $\begin{array}{l}\mathrm{D}^{-} \text {meson }(1869), \text { tau }(1776.86), \\
\text { charm anti-quark }(\approx 1275)\end{array}$ \\
\hline Saturn & $568.34(9.7 \mathrm{~km} / \mathrm{s})$ & $\begin{array}{l}491.4(-19.34 \mathrm{~km} / \mathrm{s}=- \text { Vesta orbit }) \\
477.7(-17.905 \mathrm{~km} / \mathrm{s}=- \text {-Ceres orbit })\end{array}$ & $\mathrm{K}^{-} \operatorname{meson}(493.7)$ \\
\hline Uranus & $86.813(6.8 \mathrm{~km} / \mathrm{s})$ & $\begin{array}{l}80.285(-17.89 \mathrm{~km} / \mathrm{s}=- \text { Pallas orbit }) \\
94.982(16.76 \mathrm{~km} / \mathrm{s}=\text { Hygiea orbit })\end{array}$ & $\begin{array}{l}\text { muon } \\
(\approx 95)\end{array}(105.658)$, strange quark \\
\hline Neptune & $102.413(5.43 \mathrm{~km} / \mathrm{s})$ & $96.5(-16.76 \mathrm{~km} / \mathrm{s}=-$ Hygiea orbit $)$ & $\underset{(\approx 95)}{\text { muon }}(105.658)$, strange quark \\
\hline
\end{tabular}

Table 12: Standard particle candidates for planets (green $=$ most likely) 
calculated using proper relativistic factor (Omega factor):

$$
\begin{gathered}
M_{0}=M\left[1-\left(\frac{v^{2}}{c_{n}{ }^{2}}\right)^{s}\right]^{-\frac{1}{2} q} \\
q=\operatorname{sgn}\left(c_{n}\right)=\frac{c_{n}}{\left|c_{n}\right|} \\
s=\operatorname{sgn}\left(c_{n}{ }^{2}-v^{2}\right)=\frac{c_{n}{ }^{2}-v^{2}}{\left|c_{n}{ }^{2}-v^{2}\right|} \\
\mathrm{q}=\text { sidereal polarization of the reference frame } \\
\mathrm{s}=\text { polarization of mass relative to the reference frame }
\end{gathered}
$$

where $c_{n}=c_{E H}$ is the rest velocity of the reference frame (event horizon [fossil]).

Note 1: The correlation suggests that inflation energy for these planets came individually from specific particles, with roughly equal kinetic energy. This is consistent with the hypothesized matter/anti-matter atom pair annihilation - with colliding positron/electron pairs producing the particles inflated into planets.

Such annihilation would likely occur within the gravitational maximum (event horizon) discontinuity, sending created matter and anti-matter in opposite directions, perpendicular to the maximum.

The central galactic black holes and dark matter maxima in inner/outer layers of galaxies are likely remnants of such maxima.

Note 2: Due to neutralization, there are no significant differences between planetary systems created from matter and anti-matter atoms, apart from mass distribution - if the Solar System is created from matter (a matter of convention), in an anti Solar System inner planets would have greater mass than outer planets. The reason for this is the asymmetry of space at the event horizon, where opposite charges are separated to opposite sides of the horizon. Note that this implies that horizons are, at the moment of collapse, between outer and inner planets where, after collapse, a neutrino (main dwarf planet) is formed. Note that creation of matter at event horizons resolves the missing anti-matter problem in physics - there is no anti-matter missing, there is asymmetry in mass acquisition of stable charges due to non-homogeneous energy of space, proton is anti-matter equivalent of the electron from a proper reference frame.

Thus, all positively charged particles are anti-matter particles, while negatively charged particles are matter particles (or vice versa, in alternative 
convention).

Note also that an event horizon for electron/positron annihilation can be provided by the atom nucleus itself - with the incoming electron, a maximum is extracted (expanded) from the nucleus (and possibly from the electron too) together with positron charge. At the point of annihilation, maximum (or a maximum pair) collapses with energy distributed between the created neutrino(s) and two charged particles, with none of them having enough energy to overcome escape velocity.

Due to mass asymmetry the pair will not annihilate again and the external energy (photons) is required to decouple mass and charge, and return the system to original state.

Likely, all annihilation events require expansion of particle maxima and creation of a temporary event horizon pair even if one [of larger scale] is already present at the point of annihilation.

Note 3: In CR, not only the flavors are oscillating, but, neither the rest or inertial mass is constant. Deviation from average mass is greatest in bound systems where it depends on the energy level particle occupies in the system.

Note 4: Correlation of standard masses with planetary rest mass in reference frames of orbits of bound neutrinos (main dwarf planets) is overall very good, with lower confidence only in case of the participants of the first planetary creation event - Mars and Mercury (unless the standard particle equivalent is yet to be discovered).

For Mars, the horizon at Jupiter orbit is a better fit, while for Mercury, it is the Venus orbit.

Note 5: Correlation of the Solar System with standard scale particle generations, reveals the existence of new particles in the standard model of physics (which, obviously, should be scale invariant), for example, if one interprets Saturn as $\mathrm{K}^{-}$, the Sun/Saturn mass equivalence with tau/electron reveals 2 additional standard particles:

$$
\begin{gathered}
\frac{\text { tau }}{\text { electron }} K^{-}=1717.751 \mathrm{GeV}=1.72 \mathrm{TeV} \\
\frac{\text { muon }}{\text { electron }} K^{-}=102.143 \mathrm{GeV}
\end{gathered}
$$

or, with the assumption of new energy splitting, a completely new generation (based on Sun's relativistic mass):

$$
\begin{gathered}
\frac{\text { tau }}{\text { electron }} X^{n}=3477.228 * 571.864 \mathrm{MeV}=1988.500 \mathrm{GeV}=1.9885 \mathrm{TeV} \\
\frac{\text { muon }}{\text { electron }} X^{n}=206.768 * 571.864=118.243 \mathrm{GeV}
\end{gathered}
$$


or, with Sun's proper rest mass:

$$
\begin{gathered}
\frac{\text { tau }}{\text { electron }} X^{n}=3477.228 * 537.552 \mathrm{MeV}=1869.190 \mathrm{GeV}=1.8692 \mathrm{TeV} \\
\frac{\text { muon }}{\text { electron }} X^{n}=206.768 * 537.552=111.149 \mathrm{GeV}
\end{gathered}
$$

One of these may have been discovered[20] already[21].

Evidently, using most likely particle candidates on the hypothesized particle configuration, the electric charges are in balance, as shown in Table 13. The

\begin{tabular}{l|c|c|r} 
planet & configuration & particle species $($ charge $)$ & total charge \\
\hline Mercury & $2 \mathrm{e}$ & positron $\left(1 \mathrm{e}^{+}\right)$ & $2 \mathrm{e}^{+}$ \\
Venus & $1 \mathrm{e}$ & down anti-quark $\left(1 / 3 \mathrm{e}^{+}\right)$ & $1 / 3 \mathrm{e}^{+}$ \\
Earth & $2 \mathrm{e}$ & down anti-quark $\left(1 / 3 \mathrm{e}^{+}\right)$ & $2 / 3 \mathrm{e}^{+}$ \\
Mars & $1 \mathrm{e}$ & positron $\left(1 \mathrm{e}^{+}\right)$ & $1 \mathrm{e}^{+}$ \\
Jupiter & $2 \mathrm{e}$ & charm anti-quark $\left(2 / 3 \mathrm{e}^{-}\right)$ & $4 / 3 \mathrm{e}^{-}$ \\
Saturn & $2 \mathrm{e}$ & $\mathrm{K}^{-}$meson $\left(1 \mathrm{e}^{-}\right)$ & $2 \mathrm{e}^{-}$ \\
Uranus & $1 \mathrm{e}$ & strange quark $\left(1 / 3 \mathrm{e}^{-}\right)$ & $1 / 3 \mathrm{e}^{-}$ \\
Neptune & $1 \mathrm{e}$ & strange quark $\left(1 / 3 \mathrm{e}^{-}\right)$ & $1 / 3 \mathrm{e}^{-}$
\end{tabular}

Table 13: Standard particle candidates for planets, with listed electric charges

configuration gives total $4 \mathrm{e}^{+}$charge for inner planets and $4 \mathrm{e}^{-}$for outer planets.

The fact that charge configuration agrees well with the hypothesis of 6 particles on each side (Carbon configuration) but the mass for the same particle species agrees well with 4 total particles on each side (Beryllium configuration) indicates that the original hypothesis of ${ }^{10} \mathrm{C} /{ }^{10} \mathrm{Be}$ oscillation is correct.

The fact that the sum of charges on each sides is equal to 4 , further confirms the hypothesis.

Thus, the Solar System may be interpreted as a hybrid, a superposition of 2 large scale atoms, ${ }^{10} \mathrm{C}$ and ${ }^{10} \mathrm{Be}$.

Is this hybridization unique to the inflation through annihilation of smaller scale atoms, or this is a normal state even in atoms of standard scale?

In CR, of course, the process is scale invariant and cannot be unique to one scale only, even if one cannot set up a proper reference frame to observe it.

The stability of atoms is achieved through neutral energy provided by neutral cores.

It is thus likely that all atoms are oscillating between polarized and nonpolarized states. 
Consider the case of elementary hydrogen $\left({ }^{1} \mathrm{H}\right)$.

If $1 \mathrm{e}+$ charge (ie. positron) is extracted from the nucleus to balance the electron, what prevents them from annihilating?

Obviously, between the two particles there must exist an event horizon [pair], which collapses in the interaction, forming a [bound] neutrino, but also emitting a gravitational wave of 2 maximum quanta, one of which is absorbed by the electron, the other by the positron - pushing them to stable orbits and preventing annihilation.

Note that both positron and electron are now [even more] entangled and form a standing wave.

If absorbed maxima are neutral they will increase masses of particles, decreasing charges (albeit in asymmetric manner relative to event horizon). This may be negligible but a probability exists the absorption will trigger charge [scale] collapse and mass [scale] inflation inverting the dominant nature of general force (em/gravity exchange) between the particles.

Note that, with charge extracted, proton core too becomes neutral.

It appears that [outer event horizons of] proton cores favor giving energy to electrons, while neutrons favor positrons (correlated with spin anti-alignment). Asymmetry in neutralization energy between bound positrons and electrons is thus caused in mass difference between protons and neutrons (note that magnetic fields of outer planets are much less subdued than those of inner planets).

If one interprets Neptune as the electron, Jupiter contains the mass of two down quarks, while Saturn mass has been increased with the equivalent of one up quark mass (note that charges were separated from mass before neutralization).

Assuming these masses came from protons (nucleus is scaled equally to Neptune), there are only 4 complete protons left in the nucleus. If now free up quarks [masses] couple with down quarks of a neutron, it will be converted to 2 protons. With 6 protons and 3 neutrons left, 3 more neutrons are needed to balance the core.

With a complete neutron ( 2 down quarks +1 up quark) mass on the outer side, and with remaining proton quarks left in the core, it might seem that neutralization is quantized by neutron mass.

However, the fact that Neptune and Uranus are significantly neutralized suggests that neutralization energy is correlated with quantum states and is likely scaling with element mass.

In any case, gas planets should always be the most charged ones.

Note that, with imaginary mass being dark matter and with outer planets having significant excess of gravitational potential compared to inner planets, Solar System mirrors the galaxy. 
The reason why outer planets and nearby masses are not rotating faster is due to collapse of orbital maxima into spin momenta and acquisition of real mass.

Spin coupling, in case of carbon, indicates that, as a whole system, ${ }^{12} \mathrm{C}$ is more stable than ${ }^{13} \mathrm{C}$, while ${ }^{13} \mathrm{C}$ nucleus is, due to equal number of protons and neutrons, more stable than ${ }^{12} \mathrm{C}$ nucleus.

With an excess of protons, too much energy on the outer side can cause the ejection of bound positrons and neutrinos, converting protons to neutrons.

With an excess of neutrons, too much energy on the inner side can be enough to fuse bound positrons with the nucleus [core], converting neutrons to protons.

\subsubsection{Evaluation of invariance}

Correlation between planetary masses and standard particles revealed in the previous chapter is remarkable, not only because ratios of particle masses are equal on both scales, but numeric values seem to be equal between kilograms on one scale and electron volts on another - differing only in the order of magnitude.

This reveals interesting relation between electric charge and speed of light:

$$
\begin{gathered}
e V=m \frac{c^{2}}{e} \\
m=e V K \\
e=c^{2} K
\end{gathered}
$$

where $\mathrm{K}$ on the solar system $\left(\mathrm{U}_{1}\right)$ scale is $1 * 10^{18} \mathrm{Cs}^{2} / \mathrm{m}^{2}$.

Since planetary mass is derived from GM product, integer value of $\mathrm{K}$ must be the consequence of dependence of the gravitational constant $\mathrm{G}$ on the speed of light c.

Both values, gravitational constant $\mathrm{G}$ and $\mathrm{c}$, have been determined from standard scale $\left(\mathrm{U}_{0}\right)$ experiments, thus:

$$
\begin{aligned}
G & =G_{0} \\
c & =c_{0}
\end{aligned}
$$

Mass $\mathrm{M}$ of the planet is then determined through gravitational interaction between two bodies, equalizing centripetal force with gravitational force:

$$
\begin{aligned}
\frac{m v^{2}}{r} & =\frac{G M m}{r^{2}} \\
v^{2} & =\frac{G M}{r}
\end{aligned}
$$




$$
\begin{gathered}
M=\frac{v^{2} r}{G} \\
\frac{v^{2} r}{G_{0}} \frac{1}{K}=m_{0} \frac{c_{0}^{2}}{e_{0}} \\
\frac{v^{2} r}{G_{0}} \frac{c_{1}^{2}}{e_{1}}=m_{0} \frac{c_{0}^{2}}{e_{0}}
\end{gathered}
$$

where $\mathrm{r}$ is the distance [from center] to the orbiting body [center], and $\mathrm{v}$ is its orbital velocity, and, in case of planets, also the fossil of the rest velocity of the gravitational field line (orbital maximum) before the collapse into a spin (satellite) maximum.

Planets orbiting at rest velocity are effectively at rest in the system. Since every gravitational maximum has its personal space/time - planetary orbitals are orbits of space/time within another space/time.

Equalizing centripetal force with electro-magnetic force:

$$
\begin{gathered}
\frac{m v^{2}}{r}=\frac{1}{4 \pi \epsilon_{0}} \frac{e^{2}}{r^{2}}=\frac{\mu_{0} c^{2}}{4 \pi} \frac{e^{2}}{r^{2}}=1 * 10^{-7} c^{2} \frac{e^{2}}{r^{2}} \\
m=1 * 10^{-7} \frac{c^{2}}{v^{2}} \frac{e^{2}}{r}
\end{gathered}
$$

Now equalizing $\mathrm{M}$ (gravitational mass) and $\mathrm{m}$ (charge mass):

$$
\begin{gathered}
M=m \\
\frac{v^{2} r}{G}=1 * 10^{-7} \frac{c^{2}}{v^{2}} \frac{e^{2}}{r} \\
G=1 * 10^{7} \frac{1}{c^{2} e_{0}^{2}} v_{0}^{4} r_{0}^{2}
\end{gathered}
$$

\section{$8.4 \hbar$ constant weakness}

Obvious dependency on the order of mass magnitude makes $\hbar$ a weak "constant", but at the same time explains why planetary orbits appear discrete while the orbits of small satellites seem unlimited. Obviously all masses $m>0$ must have a quantized momentum. 


\section{G relativity and equivalence with gravity}

If gravity is quantized and total mass $\mathrm{M}$ derived from gravity does not reveal quantization of angular momentum, apart from $\hbar$ scale dependence (oscillation), alternative interpretation is a variable gravitational constant G.

It is then a property of a gravitational well (maximum) and it depends on its scale.

Orbital angular momentum:

$$
M v r=n \hbar
$$

multiplied with (surface) gravity is:

$$
\begin{aligned}
& g M v r=g n \hbar \\
& g=\frac{v r}{n \hbar} g M
\end{aligned}
$$

Fixing $\mathrm{g}$ on the right side (ie. $\mathrm{M}=$ mass of Neptune, $\mathrm{g}_{0}=$ gravity of Neptune), multiplying with $\mathrm{R}^{2} / \mathrm{R}^{2}$ :

$$
g=\frac{v r}{n \hbar} g_{0} M \frac{R^{2}}{R^{2}}
$$

Fixing $\mathrm{R}$ in the numerator (ie. $\mathrm{R}_{0}=$ radius of Neptune) and equalizing with Newton gravity:

$$
g=\frac{v r}{n \hbar} g_{0} R_{0}^{2} \frac{M}{R^{2}}=\frac{G M}{R^{2}}
$$

Gravitational constant is:

$$
G=\frac{v r}{n \hbar} g_{0} R_{0}^{2}
$$

$\mathrm{v}=$ orbital velocity $\mathrm{r}=$ orbital radius

$\mathrm{R}=$ radius of the planet (spin radius)

Here, $\mathrm{v}, \mathrm{r}$ and $\mathrm{n}$ are variable. One might then consider $\hbar$ a relatively strong constant, but $\mathrm{g}_{0}$ and $\mathrm{R}_{0}$ are weak.

It has been shown that $\mathrm{g}_{0}$ alternates between two values (one taking rotation into account and one without it). The following can be concluded:

- all planets have mutually entangled properties,

- past/future state of $g_{0} / R_{0}$ is fossilized/memorized in rotation period,

- gravitational constant $\mathrm{G}$ of a gravitational well depends on its own place in a larger gravitational well.

Note that G of a planetary gravitational well is here derived form its orbital momentum in a larger well, rather than its spin momentum.

Planets are orbiting stars, but their bodies are also orbiting their souls. Mantle of a planet can be interpreted as a moon to its core, just like a moon 
can be interpreted as a collapsed gravitational maximum (event horizon) of a planet. In that system, mantle/moon is the planet and a planetary core is the star.

In case the planet is not fully developed (has active moons - in case of inner planets, or doesn't have active moons - in case of outer planets), mantle layers are asteroid belts and moons are the planets charged oppositely to the outer core of the planet.

Thus, there are gravitational constants relative to that system (note that every spin momentum is orbital momentum - even though the surface and the center are entangled, propagation of changes is not instant $=$ there are no absolute point particles).

Current value of the standard gravitational constant $\left(6.674 * 10^{-11} \mathrm{~m}^{3} / \mathrm{kgs}^{2}\right)$ was measured on Earth's surface and is relative to an absolute reference frame, proper $\mathrm{G}$ for gravitational maxima of inner planets can be obtained from surface gravity and real mass $(\mathrm{m})$ :

$$
g=\frac{\hbar_{m g}}{m}=\frac{G M}{R^{2}}
$$

Assuming speed of matter is significantly lower than the speed of space (generally valid for matter of solid bodies):

$$
m=\frac{2 \pi^{2} r_{s}^{3}}{G T_{r e}{ }^{2}}
$$

$\mathrm{m}=$ real mass of the body relative to [the scale of] its gravitational maximum

$\mathrm{r}_{s}=$ radius of the gravitational maximum $\mathrm{T}_{r e}=$ weighted average period of rotation of real mass $\mathrm{R}=$ surface radius

from (G1.1) and (G1.2) follows:

$$
\begin{gathered}
\hbar_{m g} \frac{G T_{r e}^{2}}{2 \pi^{2} r_{s}{ }^{3}}=\frac{G M}{R^{2}} \\
M=\hbar_{m g} \frac{T_{r e}{ }^{2} R^{2}}{2 \pi^{2} r_{s}{ }^{3}}
\end{gathered}
$$

with M calculated, one can now obtain G through (G1.1):

$$
G=\frac{g R^{2}}{M}=\frac{1}{\hbar_{m g}} \frac{g 2 \pi^{2} r_{s}^{3}}{T_{r e}{ }^{2}}=\frac{1}{\hbar_{m g}} \frac{g v_{r e}{ }^{2} r_{s}}{2}
$$

Note that this can also be written as:

$$
\begin{gathered}
G=\frac{1}{2} \frac{v_{r e} r_{s}}{\hbar_{m g}} g \frac{2 \pi r_{s}}{T_{r e}} \\
G=\frac{v_{r e} r_{s}}{\hbar_{m}} \hbar_{g} g \frac{\pi r_{s}}{T_{r e}}=\frac{v_{r e} r_{s}}{\hbar_{m}} \frac{v R}{n g} g \frac{\pi r_{s}}{T_{r e}}
\end{gathered}
$$




$$
G=\frac{v_{r e} r_{s}}{\hbar_{m}} \frac{\pi R^{2}}{T n} \frac{2 \pi r_{s}}{T_{r e}}=\frac{v_{r e} r_{s}}{n \hbar_{m}} \frac{2 \pi^{2} r_{s}}{T T_{r e}} R^{2}
$$

substituting middle term for $\mathrm{g}_{0}$ :

$$
\begin{gathered}
g_{0}=\frac{2 \pi^{2} r_{s}}{T T_{r e}} \\
G=\frac{v_{r e} r_{s}}{n \hbar_{m}} g_{0} R^{2}
\end{gathered}
$$

$\mathrm{v}_{r e}=$ matter (real mass) rotation speed at the gravitational maximum $\mathrm{r}_{s}$

This relation is now equivalent to the obtained relation for $\mathrm{G}$ from orbital momenta.

Note that for Earth, where $\mathrm{r}_{s}=1206115 \mathrm{~m}(\approx$ inner core radius) and $\mathrm{T}=$ $\mathrm{T}_{r e}=24 \mathrm{~h}=86400 \mathrm{~s}$ :

$$
g_{0}=0.00319 \frac{m}{s^{2}}
$$

which would match exactly the gravity of the inner core [maximum] with mass $\mathrm{M}$ equal to previously calculated real mass of Earth $\left(\mathrm{m}=6.95 * 10^{19} \mathrm{~kg}\right)$ :

$$
g_{0}=\frac{G m}{r_{s}^{2}}=0.00319 \frac{m}{s^{2}}
$$

With $\mathrm{r}_{s}$ and mass remaining constant, increase of $\mathrm{g}_{0}$ must be the result of increase in gravitational constant $\mathrm{G}$.

The current value of the gravitational constant G for Earth's maximum (with $\left.\mathrm{g}_{0}=274 \mathrm{~m} / \mathrm{s}^{2}\right)$ :

$$
G=\frac{g_{0} r_{s}^{2}}{m}=5.731534632 * 10^{-6} \frac{\mathrm{m}^{3}}{\mathrm{kgs}^{2}}
$$

Gravitational constant should not differ much between terrestrial planets. Therefor, solid real mass of these planets should have roughly equal period of rotation $\left(\mathrm{T}_{m}\right)$ to Earth's rotation period if the ratio of filled capacity to total capacity of the well is equal.

Note that real mass relative to gravitational maximum can also be calculated from equations given in CR:

$$
M=\frac{m_{r e}}{\sqrt{1-\frac{v_{r e}{ }^{2}}{c_{s}^{2}}}}+m_{i m g}
$$

with:

$$
M \sqrt{1-\frac{v_{r e}^{2}}{c_{s}^{2}}} \approx m_{i m g}
$$

real mass is:

$$
m=m_{r e}=\left(1-\sqrt{1-\frac{v_{r e}^{2}}{c_{s}^{2}}}\right) m_{i m g}=6.95 * 10^{19} \mathrm{~kg}
$$


$\mathrm{c}_{s}=$ angular velocity of [space] of the gravitational maximum $=18178.98 \mathrm{~m} / \mathrm{s}$

$$
\mathrm{m}_{i m g}=\mathrm{M}=5.9723 * 10^{24} \mathrm{~kg}
$$

From the perspective of a maximum, obtained real mass is the mass of all acquired matter (from our perspective, real mass $=\mathrm{M}=5.9723 * 10^{24}$ ) $\mathrm{kg}$ ).

From our perspective, real mass relative to the maximum should be interpreted as unused capacity of the well. The slower the rotation of real mass of a terrestrial planet, the less free capacity for real mass there is.

The slower the rotation of acquired real mass, the greater is the difference in velocity between the [space of a] maximum and real mass. The increasing relative retrograde motion of real mass is creating pressure on the maximum. While the capacity cannot be completely full for more than 0 time, the less free capacity there is the less energy is needed to trigger the collapse (spin change) of the maximum. Death, thus, becomes more likely.

Note that, by the equation above, the capacity cannot be completely full. This cannot be true absolutely. If rest real mass is increased externally by a pulse of energy, the well can even become overcapacitated. However, from the perspective of a maximum, once the well is full, relative infinity (singularity) is reached and it must collapse.

This can be interpreted as slowing down of the rotation of the maximum (decreasing $\mathrm{c}_{s}$ in the equation). While the equation will eventually give absolute infinity there is no infinite energy required. The energy may remain relatively constant, but with such changes in spin momenta, the body of real mass and maximum are simply loosing entanglement and the equation becomes irrelevant (invalid) - the body will get entangled with another maximum, possibly changing $\mathrm{c}_{s}$ in the equation.

This is true for all relativistic equations - changes in angular momenta can produce relative infinities and loss of entanglement between frames. Note that, this is a variant of a Pauli exclusion principle - in this case, however, it is anti-alignment of spin momenta that is not allowed for stable coupling of bodies - in this case, this is a coupling between a body of matter (real mass) and a gravitational maximum (or, a body of dark matter - with its gravitational well included).

\subsection{Evidence for a constant change of $\mathrm{G}$}

If $\mathrm{G}$ is variable, it should generally increase at the expense of the Coulomb constant, although changes in space cannot be instant and some phase shift at distance will exist.

In a bound configuration such as a Solar System, change in G of local space will be reflected in changes of orbital momentum.

Taking mass and distance into account, major influence on G on Earth is the 
interaction with the Sun (multiple orders of magnitude larger than the Moon and planets).

The G constant should thus oscillate, with the 1st order sinusoidal oscillation due to Earth's elliptical orbit of the Sun.

In the 6p4n configuration of the Solar System, the existence of a perihelion and aphelion in the Earth's orbit is reflected in two discontinuities of the Sun, at $2 / 3 \mathrm{R}$ and $1 / 2 \mathrm{R}$.

With a change in distance from the Sun, spin velocity of Earth's maximum is changing relative to the rest frame of the two discontinuities - it's radius is expanding and contracting, directly affecting the local G constant.

Mean change of $\mathrm{G}$ due to perihelion is thus:

$$
\Delta G_{p}=\frac{1}{2}\left(\frac{\sqrt{1-\frac{v^{2}}{c_{1.1} 1^{2}}}}{\sqrt{1-\frac{v_{p}^{2}}{c_{1.1}}}}+\frac{\sqrt{1-\frac{v^{2}}{c_{1.2}}}}{\sqrt{1-\frac{v_{p}^{2}}{c_{1.2}}}}\right)=1.0002446
$$

Change of $\mathrm{G}$ due to aphelion:

$$
\Delta G_{a}=\frac{1}{2}\left(\frac{\sqrt{1-\frac{v_{a}^{2}}{c_{1.1}^{2}}}}{\sqrt{1-\frac{v^{2}}{c_{1.1^{2}}}}}+\frac{\sqrt{1-\frac{v_{a}^{2}}{c_{1.2}{ }^{2}}}}{\sqrt{1-\frac{v^{2}}{c_{1.2}}}}\right)=1.0002354
$$

Giving the total:

$$
\Delta G=\frac{1}{2}\left(\Delta G_{p}+\Delta G_{a}\right)=1.00024
$$

$\mathrm{v}=$ orbital velocity of Earth at semi-major axis $=29784.485 \mathrm{~m} / \mathrm{s}$ $\mathrm{v}_{p}=$ orbital velocity of Earth at perihelion $=30037.537 \mathrm{~m} / \mathrm{s}$ $\mathrm{v}_{a}=$ orbital velocity of Earth at aphelion $=29538.694 \mathrm{~m} / \mathrm{s}$ $\mathrm{c}_{1.2}=$ space orbital velocity of the $1 / 2 \mathrm{R}$ Sun discontinuity $=151.266563 *$ $10^{3} \mathrm{~m} / \mathrm{s}$ $\mathrm{c}_{1.1}=$ space orbital velocity of the $2 / 3 \mathrm{R}$ Sun discontinuity $=230.556106 *$ $10^{3} \mathrm{~m} / \mathrm{s}$

Velocities $\mathrm{c}_{1.1}$ and $\mathrm{c}_{1.2}$ have been calculated in the Quantization of the Sun chapter.

For a mean $\mathrm{G}$ of $6.673899 * 10^{-11} \mathrm{~m}^{3} / \mathrm{kgs}^{2}$ and $\Delta \mathrm{G}=1.00024$, the amplitude of oscillation is $1.60173576 * 10^{-14} \mathrm{~m}^{3} / \mathrm{kgs}^{2}$.

Measurements of $\mathrm{G}$ on Earth indeed show sinusoidal oscillation, although in previous analysis it has been correlated with the $5.9 \mathrm{y}(5.899 \pm 0.062 \mathrm{y})$ period oscillation component of Earth's length of day (LOD) [22].

However, calculated amplitude of yearly oscillation $\left(1.60173576 * 10^{-14}\right.$ $\left.\mathrm{m}^{3} / \mathrm{kgs}^{2}\right)$ agrees very well with the amplitude obtained from measurements $\left(1.619 \pm 0.103 * 10^{-14} \mathrm{~m}^{3} / \mathrm{kgs}^{2}\right)$. 


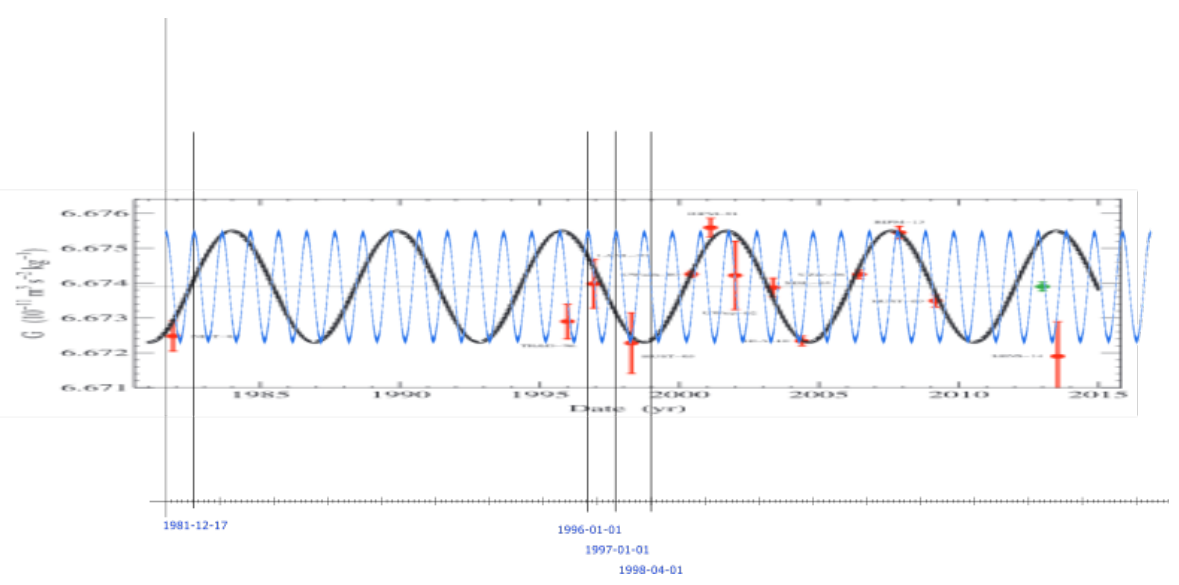

Figure 10: Oscillation of the gravitational constant

Fig. 10 shows yearly oscillation (blue) superimposed on the $5.9 y$ oscillation from previous analysis (black). Red crosses are previously measured values of G, plotted with uncertainties.

Yearly oscillation is obviously a better fit, but when linked to orbits of the Earth around the Sun (orbital data taken from NASA Horizons On-Line Ephemeris System[23]) a phase shift of $\approx 0.6167 \mathrm{y}$ (golden ratio?) is required to match Fig. 10 (without the shift the correlation is less convincing with all measurements taken into account).

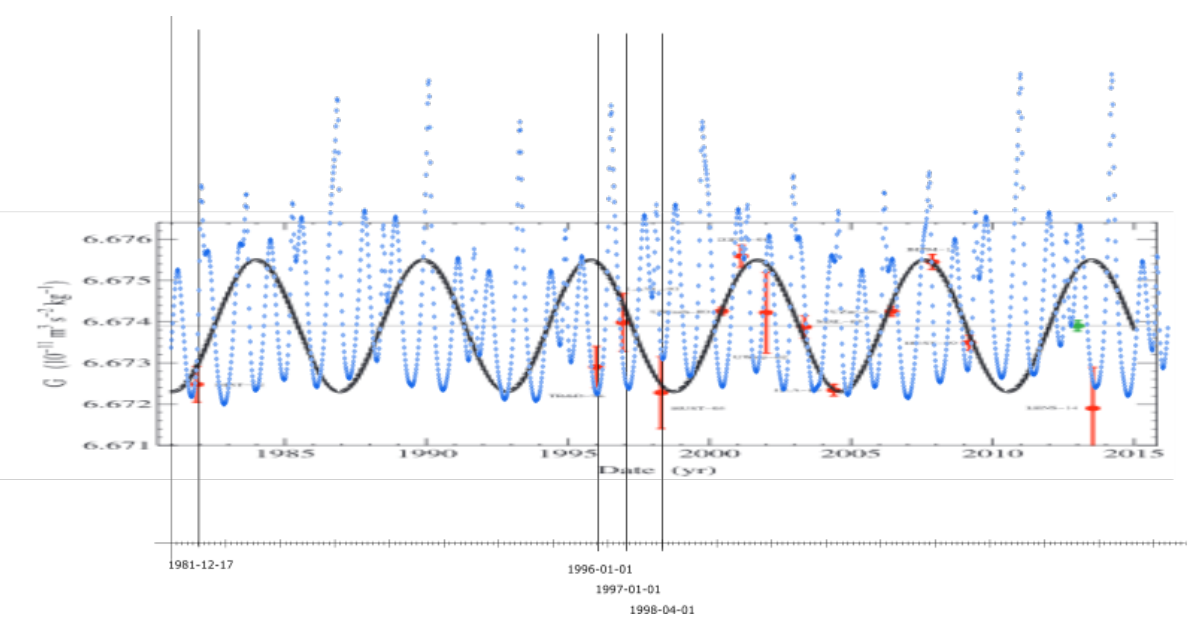

Figure 11: Oscillation of the gravitational constant

Interestingly, as shown on Fig. 11, with the influence of the Sun removed, leaving only planetary gravitational interactions, best fit requires no phase shift. 
The 5.9y period oscillation in LOD is equal to a solar orbit in 2:1 resonance with Jupiter and a 5:1 resonance with Saturn. If Mars (which is in 1e configuration) is entangled with 1e of Jupiter, the Earth (2e configuration) should be entangled with the other 1e of Jupiter and 1e of Saturn.

The resonant orbital (outer edge of the main asteroid belt) must be the event horizon (which should currently be in a collapsed form - similar to larger horizons collapsed into dwarf planets) of such entanglements.

This is (or rather, a memory of - due to neutralization of EM force) a magnetic spin entanglement between particles (notice the anti-alignment of magnetic fields between Earth and Jupiter/Saturn), and thus should have a signature in geomagnetic field.

\section{Quantization of surface radii}

Here are, somewhat empirically determined, equations for quantization of surface radii in the Solar System.

Neutral equatorial radius for outer planets:

$$
R=\frac{K_{2}}{r^{2}} M \frac{1}{2^{(2-p)}}\left[\left(\frac{1}{10^{1}}\right)^{(4-N)} 3^{(3-p)} \frac{1}{n^{(p-1)}}\right]^{(s-1)}
$$

Neutral equatorial radius for inner planets:

$$
R=\frac{r^{2}}{K_{1}} \frac{1}{M} n^{(1-p)} 2^{(N-1)}\left[2^{(4-n)} \frac{1}{3^{(1-p)}}\right]^{(s-1)}
$$

Since both $\mathrm{r}$ and $\mathrm{M}$ (gravity) are quantized, it follows that $\mathrm{R}$ is quantized too by the K constant - other factors (n, p, s, N) are integers.

The above may be understood as the invariant component of the radius during the cycle. Current radius includes a small correction due to oscillation in electric polarization (charge), value of which evolves weakly during the cycle state.

Current equatorial radius for outer planets:

$$
\begin{aligned}
& R=\frac{K_{2}}{r^{2}} M \frac{1}{2^{(2-p)}} \frac{1}{10^{1}}\left(\frac{3^{2}}{10^{1}}\right)^{\left(2-K_{\varphi}\right)} K_{\varphi}\left[\frac{1}{n^{(p-1)}}\right]^{(s-1)} \\
& K_{\varphi}=10^{-\left[\sin \left(180^{\circ}-\Delta_{\varphi}\right)^{(p \bmod 2)} \cos \left(180^{\circ}-\Delta_{\varphi}\right)^{(1-p \bmod 2)}\right]}
\end{aligned}
$$




$$
\Delta_{\varphi}=\varphi_{0}-\varphi_{1}
$$

Current equatorial radius for inner planets:

$$
\begin{aligned}
& R=\frac{r^{2}}{K_{1}} \frac{1}{M} n^{(1-p)}\left(2+K_{\varphi}\right)\left[2^{(2-p)} 10^{0}\left(\frac{10^{0}}{3^{2}}\right)^{-K_{\varphi}}\right]^{(s-1)} \\
& K_{\varphi}=10^{0} \cos \left(180^{\circ}-\Delta_{\varphi}\right)^{(p \bmod 2)} \sin \left(180^{\circ}-\Delta_{\varphi}\right)^{(1-p \bmod 2)} \\
& \Delta_{\varphi}=\varphi_{0}-\varphi_{1} \\
& \mathrm{~K}_{2}=4885811.341 \mathrm{~m}^{3} / \mathrm{kg} \\
& \mathrm{K}_{1}=2.385039177^{*} 10^{-9} \mathrm{~m} / \mathrm{kg} \\
& \mathrm{M}=\text { total mass } \\
& \mathrm{r}=\text { orbital radius } \\
& \mathrm{N}=\text { shell number } \\
& \mathrm{s}=\text { number of particles in a sub-shell } \\
& \mathrm{p}=\text { state of quantization } \\
& \mathrm{n}=\text { shell energy level }
\end{aligned}
$$

Calculated radii for the state $6 \mathrm{p} 4 \mathrm{n}$ are shown in Table 14 .

\begin{tabular}{c|c|c|c|c|c|c|c|l|l|l}
$\mathrm{N}$ & $\mathrm{n}$ & planet & $\mathrm{M}(\mathrm{kg})$ & $\mathrm{r}\left(10^{6} \mathrm{~km}\right)$ & $\mathrm{s}$ & $\mathrm{p}$ & $\Delta_{\phi}\left(^{\circ}\right)$ & $\begin{array}{l}\text { neutral } \\
\mathrm{R} \\
(\mathrm{km})\end{array}$ & $\begin{array}{l}\text { current } \\
\mathrm{R} \\
(\mathrm{km})\end{array}$ & $\mathrm{R}(\mathrm{km})$ \\
\hline 2 & 5 & Neptune & $1.02413 * 10^{26}$ & 4495.06 & 1 & 2 & 36.7084 & 24764 & 24764 & 24764 \\
2 & 5 & Uranus & $8.6813 * 10^{25}$ & 2872.46 & 1 & 1 & 233.1506 & 25703 & 25559 & 25559 \\
2 & 3 & Saturn & $5.6834 * 10^{26}$ & 1433.53 & 2 & 1 & 0.2 & 60806 & 60268 & 60268 \\
1 & 1 & Jupiter & $1.89819 * 10^{27}$ & 778.57 & 2 & 1 & 109.422 & 68848 & 71492 & 71492 \\
2 & 5 & Mercury & $3.3011 * 10^{23}$ & 57.91 & 2 & 2 & 172.3047 & 2555.7 & 2439.7 & 2439.7 \\
2 & 3 & Venus & $4.8675 * 10^{24}$ & 108.21 & 1 & 0 & 0 & 6051.8 & 6051.8 & 6051.8 \\
2 & 3 & Earth & $5.9723 * 10^{24}$ & 149.60 & 2 & 1 & 90.3135 & 6284.72 & 6378.14 & 6378.14 \\
1 & 10 & Mars & $6.4171 * 10^{23}$ & 227.92 & 1 & 2 & -91.9957 & 3394.1 & 3396.2 & 3396.2
\end{tabular}

Table 14: Calculated neutral and current radii

Note the quantization of $\Delta_{\phi}$. For inner planets, it is quantized by $90^{\circ}$ (any deviation may be due to higher order oscillation).

For outer planets, the quantum is reduced to $1 / 5$ of this value, $18^{\circ}$, suggesting, perhaps that the equation for outer planets should be modified, or, instability in entanglement.

Thus, to obtain $90^{\circ}$ quantization, one only needs to multiply $\Delta_{\phi}$ (quantized by $18^{\circ}$ ) with 5 , revealing how it is entangled (assuming anti-alignment) with one of the inner planets, as shown in Table 15. Here, Neptune/Venus, Uranus/Mars and Saturn/Mercury entanglement should not be surprising due to matching configurations - 1e/1e, $1 \mathrm{e} / 1 \mathrm{e}$ and $2 \mathrm{e} / 2 \mathrm{e}$.

The entanglement of Jupiter with Venus instead of Earth might be the consequence of ${ }^{10} \mathrm{C}$ instability, or a phase shift in entanglement. 


\begin{tabular}{l|c|r} 
planet & standardized $\Delta_{\phi}\left(^{\circ}\right)$ & entanglement \\
\hline Neptune & $(5 * 36) \% 360=180$ & Venus \\
Uranus & $(5 * 234) \% 360=90$ & Mars \\
Saturn & $(5 * 0) \% 360=0$ & Mercury \\
Jupiter & $(5 * 108) \% 360=180$ & Venus
\end{tabular}

Table 15: Correlation of outer and inner planets

\subsection{Radius of the Sun and its correlation with proton radius}

Original composition of the Sun is 6 protons +4 neutrons. However, 6 positrons worth of charge (inner planets) have been removed to balance the electrons (outer planets).

This makes the Sun neutral:

$$
6 *\left(-\frac{1}{3} e+2 * \frac{2}{3} e\right)+4 *\left(2 *-\frac{1}{3} e+\frac{2}{3} e\right)-6 e=0
$$

The Sun still consists of both positive and negative charges but their spin effects on radius cancel out. The radius is thus:

$$
\begin{gathered}
R=R_{2}+R_{1} \\
R_{2}=\frac{K_{2}}{r_{2}{ }^{2}} M_{2} \frac{1}{2^{\left(2-p_{2}\right)}}\left[\left(\frac{1}{10^{1}}\right)^{\left(4-N_{2}\right)} 3^{\left(3-p_{2}\right)} \frac{1}{n_{2}^{\left(p_{2}-1\right)}}\right]^{\left(s_{2}-1\right)} \\
R_{1}=\frac{r_{1}^{2}}{K_{1}} \frac{1}{M_{1}} n_{1}^{\left(1-p_{1}\right)} 2^{\left(N_{1}-1\right)}\left[2^{\left(4-n_{1}\right)} \frac{1}{3^{\left(1-p_{1}\right)}}\right]^{\left(s_{1}-1\right)}
\end{gathered}
$$

where $R_{2}$ is the sum radius of negative quarks and $R_{1}$ is the sum radius of positive quarks.

As shown on Fig. 12, without 6 +e charges, the Sun is a sum neutron consisting of 6 layers, 4 layers containing pairs of negative [down equivalent] quarks and 2 layers (inner and outer core) containing pairs of positive [up equivalent] quarks. Due to condensation, this is the equivalent of a single neutron so 8 negative quarks can be grouped into a single sub-shell as 2 negative quarks, while 4 positive quarks can be grouped into another sub-shell as a single positive quark $(8 / 4=2 / 1)$.

Thus, the parameter $\mathrm{s}_{2}=2$, while $\mathrm{s}_{1}=1$.

The energy of these two sub-shells must be equal, so $\mathrm{M}_{2}=\mathrm{M}_{1}=\mathrm{M}$.

For equal impact on radii, this must be satisfied:

$$
\frac{K_{2}}{r_{2}^{2}} M_{2}=\frac{r_{1}^{2}}{K_{1}} \frac{1}{M_{1}}
$$




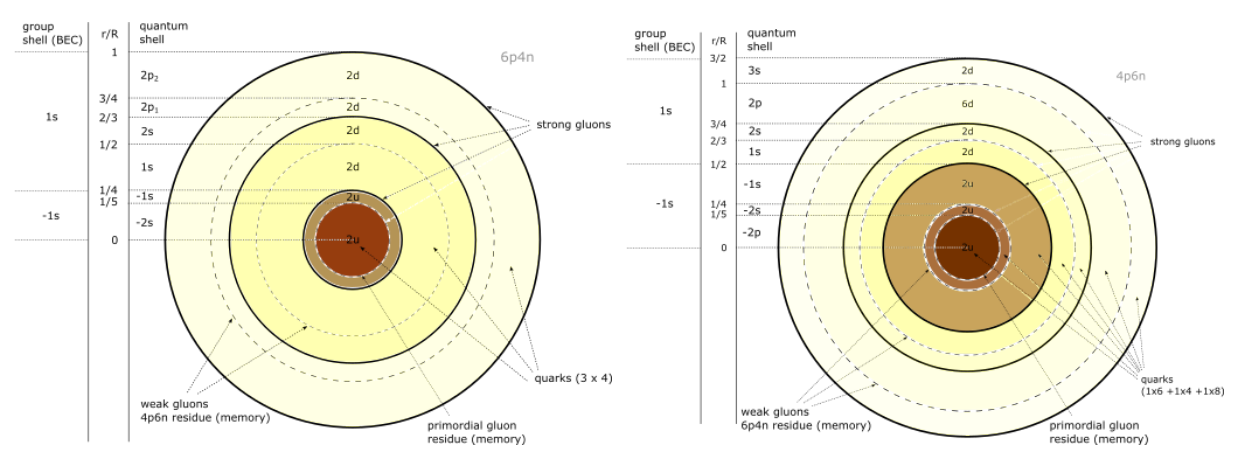

Figure 12: Sun partitioning in: a) $6 \mathrm{p} 4 \mathrm{n}$ state b) $4 \mathrm{p} 6 \mathrm{n}$ state $(\mathrm{R}=$ radius in $6 \mathrm{p} 4 \mathrm{n}$ state)

Since $\mathrm{M}_{2}=\mathrm{M}_{1}=\mathrm{M}$ :

$$
\begin{gathered}
\frac{K_{2}}{r_{2}^{2}} M=\frac{r_{1}^{2}}{K_{1}} \frac{1}{M}=\sqrt{\frac{K_{2}}{K_{1}}} \frac{r_{1}}{r_{2}} \\
R=\sqrt{\frac{K_{2}}{K_{1}}} \frac{r_{1}}{r_{2}}\left[\frac{1}{2^{\left(2-p_{2}\right)}}\left(\frac{1}{10^{1}}\right)^{\left(4-N_{2}\right)} 3^{\left(3-p_{2}\right)} \frac{1}{n_{2}^{\left(p_{2}-1\right)}}+n_{1}^{\left(1-p_{1}\right)} 2^{\left(N_{1}-1\right)}\right]
\end{gathered}
$$

Here $\mathrm{p}_{1}$ corresponds to number of major (strong) gluons, $\mathrm{p}_{2}$ to weak gluons, $\mathrm{N}$ continues increasing from Mercury (2) so $\mathrm{N}_{1}=3$ and $\mathrm{N}_{2}=4$ :

$$
\begin{gathered}
p_{2}=2, N_{2}=4, n_{2}=3^{2}=9 \\
p_{1}=3, N_{1}=3, n_{1}=3
\end{gathered}
$$

Sun radius then becomes:

$$
R=\sqrt{\frac{K_{2}}{K_{1}}} \frac{r_{1}}{r_{2}}\left[\frac{1}{3}+\left(\frac{2}{3}\right)^{2}\right]
$$

Here, ratio $r_{1} / r_{2}$ is equal to the ratio of orbital radii of the outermost electron (Neptune) and the outermost positron (Mars).

This gives $\mathrm{R}=694271.2405 \mathrm{~km}$.

Radius of the sum $\mathrm{U}_{1}$ scale proton can be obtained by raising the quark factors of $\mathrm{R}$ to the power of 2 . This is due to the fact that the removal of a negative down quark reduces the negative radius $9\left(3^{2}\right)$ times, while the addition of a positive up quark reduces the positive radius $3 / 2$ times. Distance between charges increases (due to greater difference between them) so total radius is decreased by the sum of these factors. 


$$
R_{p_{1}}=\sqrt{\frac{K_{2}}{K_{1}}} \frac{r_{1}}{r_{2}}\left[\frac{1}{3}+\left(\frac{2}{3}\right)^{2}\right]\left[\left(\frac{1}{3}\right)^{2}+\frac{2}{3}\right]=\sqrt{\frac{K_{2}}{K_{1}}} \frac{r_{1}}{r_{2}}\left[\frac{1}{3}+\left(\frac{2}{3}\right)^{2}\right]^{2}
$$

Radius of a standard proton ( $\mathrm{U}_{0}$ scale) can now be obtained through this equation:

$$
\frac{R_{p_{1}}}{r_{1}}=\frac{N * R_{p}}{r_{U_{0}}}
$$

Where $r_{1}$ is the Solar System charge radius (Neptune's orbit), $N$ is the number of nucleons in the Solar System, $\mathrm{R}_{p}$ is the standard proton radius and $\mathrm{r}_{U_{0}}$ is the standard Carbon-10 (Carbon-12) charge radius.

Using Sun radius $\mathrm{R}$ obtained above, this gives:

$$
R_{p}=R_{p_{0}}=\frac{1}{10} \frac{R}{r_{1}}\left[\left(\frac{1}{3}\right)^{2}+\frac{2}{3}\right] r_{U_{0}}=0.840905616 * 10^{-15} \mathrm{~m}
$$

\section{2 $\Delta_{\phi}$ validation}

Dominant magnetic field in outer planets may be generated by positive charge, while in inner planets by negative.

In any case, $\Delta_{\phi}$ may also correspond to angle between magnetic dipoles.

\subsubsection{Mercury}

$\Delta_{\phi}$ obtained for Mercury corresponds to $\downarrow \uparrow$ spin configuration. This is generally consistent with a low strength magnetic field. However, current low strength of Mercury's magnetic field should not be attributed to such configuration as the primal source is subdued.

\subsubsection{Venus}

$\Delta_{\phi}$ for Venus suggests extremely strong primal magnetic field.

\subsubsection{Earth}

Earth's magnetic dipole is not axial, revealing a primal quadrupole configuration, as expected with 2e configuration. Considering the movement of north and south dip poles and attributing it to imminent collapse, in the primal configuration two major (inner and outer) dipoles may very well be separated by $90^{\circ}$, equal to calculated $\Delta_{\phi}$.

This configuration may have been fossilized in the inner core anisotropy, as shown on Fig. 13. 


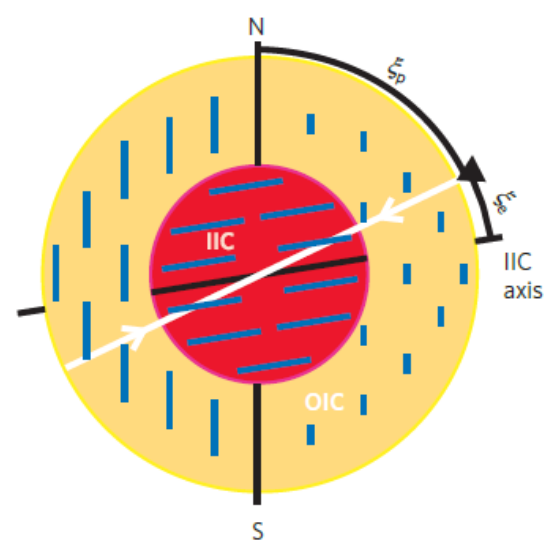

Figure 13: Equatorial anisotropy of the Earth's inner core ${ }^{24}$

\subsubsection{Mars}

Obtained $\Delta_{\phi}$ shows primal dipole configuration of Mars is mirroring the Earth's. The configuration may be verified once the magnetic field is restored.

\subsubsection{Jupiter}

$\Delta_{\phi}\left(109^{\circ}\right)$ corresponds to $\uparrow \rightarrow$ configuration, and is consistent with observation, as shown on Fig. 14.

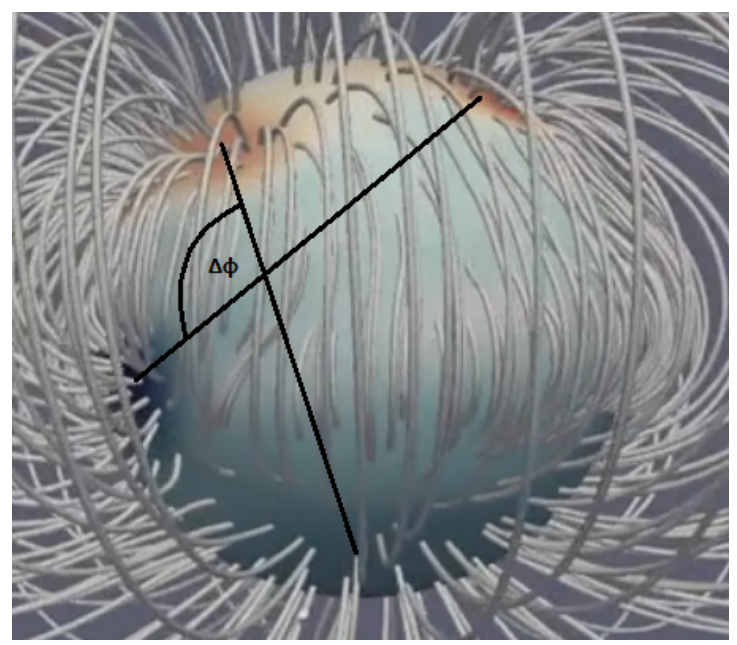

Figure 14: Magnetic field of Jupiter ${ }^{25}$ 


\subsubsection{Saturn}

Saturn's dipole field is aligned with the rotation axis and highly axisymmetric, while quadrupole and higher components are significantly weaker.

This is consistent with $\uparrow \uparrow$ configuration suggested by $\Delta_{\phi}\left(0^{\circ}\right)$.

\subsubsection{Uranus}

Dipole center is significantly offset from the center of the planet. Assuming primal core-dipole entanglement, $\Delta_{\phi}$ may be interpreted as the angle between the equator and dipole rotated by such angle that the [shortest] distance from dipole center to equator ( $\mathrm{x}$ ) is equal to distance from planet surface to the intersection of the rotated axis and axis translated to center, as shown on Fig. 15.

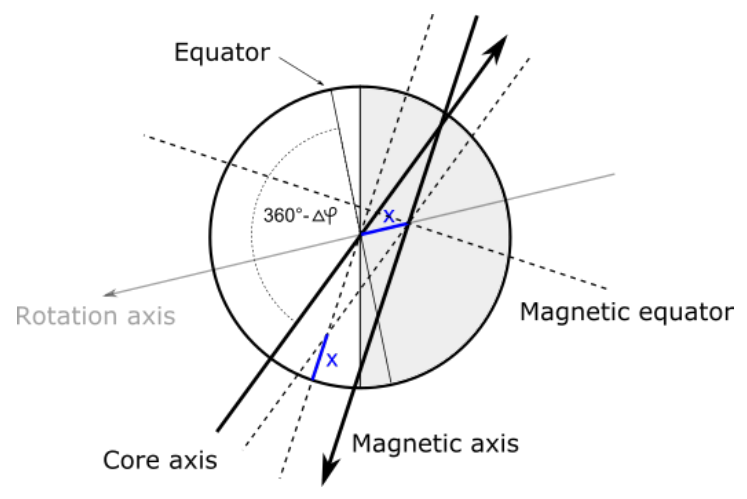

Figure 15: Uranus' magnetic field model

With an $58.6^{\circ}$ tilt of the dipole from rotational axis and no inclination, the offset $=\mathrm{x}=0.38192 \mathrm{R}$.

With an inclination of the dipole from rotational axis equal to $1.82^{\circ}$, the offset is equal to $0.353 \mathrm{R}$, in agreement with $\mathrm{NASA} / \mathrm{GSFC}^{-\mathrm{O}_{3}} \operatorname{model}[26]$.

\subsubsection{Neptune}

Similar to Uranus, the dipole is significantly offset from the center. Using the same method as in case of Uranus, one obtains the dipole shown on Fig. 16.

With an $46.9^{\circ}$ tilt of the dipole from rotational axis, with no inclination, the offset is equal to $0.12193 \mathrm{R}$.

With an inclination of $63.2716^{\circ}$, the offset $=y=0.485 \mathrm{R}(\mathrm{x}=0.244967695$ R), equal to NASA/GSFC-O 8 model[27] offset.

\section{Earth, as a particle}

Orbits of planetary gravitational maxima and their capacities are quantized. 




Figure 16: Neptune's magnetic field model

It would be intuitive to expect that gravity within the planet follows the same pattern.

For positive bodies (terrestrial planets), gravity should generally increase with depth, down to the inner gravitational maximum, which may have the radius of the inner core at full capacity.

For Earth, gravity of this maximum is equal to the surface maximum of the Sun $-274 \mathrm{~m} / \mathrm{s}^{2}$.

For a naked maximum, gravity down to the maximum:

$$
\begin{gathered}
g v r=n h \\
g r^{2}=n T \frac{h}{2 \pi} \\
g r^{2}=n T \hbar, \\
g=n T \frac{\hbar}{r^{2}},
\end{gathered}
$$

where $\mathrm{T}$ is the rotation period at radius $\mathrm{r}$. In equilibrium, $\mathrm{T}$ at surface for a solid body is:

$$
T=T_{0},
$$

while real radius of the planet is:

$$
R=R_{0}
$$

If $\mathrm{nT}$ is const.:

$$
g=\frac{\hbar_{m g}}{m} \frac{R^{2}}{r^{2}}
$$


For Earth:

$$
\begin{gathered}
T=24 h=86400 \mathrm{~s} \\
R_{0}=\text { const. }=6307105 \mathrm{~m}
\end{gathered}
$$

Down to the inner core radius $r_{c}$ :

$$
\begin{gathered}
n=n_{s}=1 \\
g=86400 \frac{\hbar}{r^{2}}=\frac{G M}{r^{2}} \\
\mathrm{G}=\text { gravitational constant }=6.674 * 10^{-11} \mathrm{~m}^{3} / \mathrm{kgs}^{2} \\
\quad \hbar=\hbar_{1}=4.613325255 * 10^{9} \mathrm{~m}^{3} / \mathrm{s}^{3}
\end{gathered}
$$

\begin{tabular}{l|c|c|r}
$\mathrm{n}$ & discontinuity & radius $(\mathrm{m})$ & gravity $\left(\mathrm{m} / \mathrm{s}^{2}\right)$ \\
\hline 1 & crust surface & 6371000 & 9.82 \\
1 & crust surface perihelion & 6357000 & 9.86 \\
1 & real surface & 6307105 & 10.02 \\
1 & outer core & 3282185 & 37 \\
1 & transition zone (induced charge) & 1705704 & 137 \\
1 & g. maximum = inner core radius $=\mathrm{r}_{c}$ & 1206115 & 274 \\
1 & transition zone & 852852 & 137 \\
$2 ?$ & inner inner core & 603058 & $274 ?$
\end{tabular}

Table 16: Gravity of [naked] Earth

Below $\mathrm{r}_{c}(1206115 \mathrm{~m})$ gravity becomes:

$$
g=n^{2} \frac{1}{T} \frac{1}{\hbar_{2}} r^{2}
$$

At $r_{c}$ (event horizon):

$$
n T \frac{\hbar_{1}}{r^{2}}=n^{2} \frac{1}{T} \frac{1}{\hbar_{2}} r^{2}, \rightarrow \hbar_{2}=6.144878706 * 10^{4} \mathrm{~ms}
$$

At the event horizon gravity is independent of period and radius:

$$
g=\sqrt{\frac{\hbar_{1}}{\hbar_{2}}}
$$

Below $r_{c}$ the space-time gradient inverts and gravity is decreasing until it reaches minimum, afterwards increasing again to next maximum, continuing the oscillation. 
I am not particularly happy correlating period of rotation of matter to gravity. In this case, it can be interpreted as a constant of proportionality, and, as such, it works.

Generally, gravity should be correlated with rotation of space (generally, Keplerian velocity).

\subsection{Gravity with acquired matter}

A gravitational maximum (soul) will effectively curve space around it. This space has pressure and density [gradients] and acquired matter will not affect the overall curvature of that space as long as gravity of the maximum is greater than gravity of acquired matter (in which case, space of matter will be strongly localized).

This is why gravity at Earth's surface is roughly the same as it was when the maximum was naked. However, clumping or condensation of matter (nonhomogeneous system) can produce measurable effects.

In, addition, core maximum may split into multiple maxima which may further collapse to form orbiting spin momenta.

In case of Earth, presence of mantle discontinuities suggests presence of multiple maxima. Organization of matter into layers and lack of large gravitational anomalies (no strong deviation from spherical space) also suggests none of these have collapsed (unless Moon's maximum is understood as collapsed Earth maximum, however, Moon's maximum is more likely to expand and integrate into Earth in the future).

Distribution of gravity inside Earth is thus likely wavelike, being canceled at least at some discontinuities, albeit with overall increasing density toward the core.

This enables Earth's mantle to have layers where pressures and temperatures are suitable for complex life.

\section{Earth, as a living organ(ism)}

Earth is not only behaving as a living organism, it is a living organism in the same sense as any life-form on its surface is a living organism.

Solar System is also an individual organism, and, relative to that system, Sun and planets are the organs.

Obeying the principle of self-similarity, each living organ has an active core, replicating the role of the Sun in the Solar System to localized space-time.

These are all extremely introverted organisms. For that reason, creatures of extroverted nature may not recognize them as living beings, however, lack of complexity in physical momenta is simply replaced with complexity in mental momenta - which is reflected in momenta of smaller scale life-forms residing 
inside their bodies. One of these life-forms are humans, who are, relative to Earth, its [precursor] proteins.

\subsection{Rough internal structure}

Earth is an extremely introverted life-form. As such, it has no need for limbs and complexity in organ structure, albeit smaller components of these structures can be complex.

Most expressed organ of this organism is the brain, organized into layers with minimal gyrification.

Gyrification of tissue is present in standard complex life only due to presence of organs required for extroverted interaction (eyes, nose, ears, mouth, body from the neck down).

Even so, it has to have other organs [or organ equivalents] necessary for the function of that brain.

Most likely blood arteries are underground tubes, with blood being the flowing magma and water (nutrients).

Proper interpretation of lava solidification is thus coagulation of blood.

Its veins are underground tubes filled with oil (pressurized dead carbon matter).

Note that, unlike human blood, Earth's arteries do not carry dissolved oxygen while veins do not carry dissolved carbon dioxide (at least not in high concentrations near surface).

Rather, they carry bound oxygen and carbon, which are then used as fuel to produce molecular oxygen and carbon dioxide where needed.

It is possible, however, that within the mantle, arteries and veins do carry significant amounts of dissolved gases.

Complex life and networks of interconnected diversity are not limited to surface (epidermis). In fact, surface is likely just a breeding ground for cultivation of precursor neuron cells and proteins of a planet. Most complex life is thus resident within mantle layers where it is protected and not so vulnerable to external influence.

The core of a planet has a role of the heart and geyser eruptions provide one way to probe the heart rate. 


\subsection{Age, lifespan and 3rd order period}

The lifetime of Earth is quantized and can be calculated through its frequency of existence.

$$
\Delta T_{E}=n \frac{1}{f_{x}}=n T_{x}
$$

For $\mathrm{n}=2840$, and determined $\mathrm{T}_{x}$ of the 3rd order general oscillation of the Solar System equal to $1.512 * 10^{6}$ years:

$$
\Delta T_{E}=n T_{x}=4.29408 * 10^{9} \text { years }
$$

But this should not be interpreted as lifespan of Earth, rather lifespan of the Solar System. Earth's lifespan is obtained with $\mathrm{n}=1$. It is thus equal to $\mathrm{T}_{x}$.

Lifetime and lifespan are different for bodies on $\mathrm{U}_{1}$ scale, such as planets. This is because, with expiration of lifespan, the body is generally reused by another soul (gravitational maximum). Lifetime (age) for planets will thus be generally larger than lifespan. Lifetime may be interpreted as the age of the body, while lifespan is the average lifespan of the soul in a particular system.

There are at least 3 ways to calculate the 3rd order period of existence cycle $\mathrm{T}_{x}$ [and thus, Earth's lifespan], all giving the same result:

\subsubsection{Decay rate of $10 \mathrm{C}$ at $\mathrm{U}_{1}$ scale}

Current Solar System state is ${ }^{10} \mathrm{C}$. Half-lives of elements are inverted relative to the shared decay product between adjacent vertical scales and fossilized as the 3rd order period of oscillation.

Thus, the $3 \mathrm{rd}$ order period of the $\mathrm{U}_{1}{ }^{10} \mathrm{C}$ is equal to the half-life of ${ }^{10} \mathrm{Be}$ at $\mathrm{U}_{0}$ scale.

Several measurements of ${ }^{10} \mathrm{Be}$ half-life have been performed.

In example, in 1987. it was measured to be $1.51 \pm 0.06 * 10^{6}$ years[28].

In 2009. it was measured to be $1.388 \pm 0.018 * 10^{6}$ years[29].

Even though half-life of $\mathrm{U}^{0}$ elements should be consistent during the existence cycle of $\mathrm{U}^{1}$, it changes during the transition between cycles - $\mathrm{T}_{x}$ should be understood as the mean value.

For that reason, I do not consider the value from 2009. as the average value through the lifetime of the Solar System. It will be shown later that this value is $1.512 * 10^{6}$ years.

This is the 3rd order period of the existence cycle of the Solar System, and consequently, Earth. 


\subsubsection{Heart rate}

The average heart rate of Earth can be calculated from the global average period between geyser eruptions:

$$
\left\langle T_{g}\right\rangle_{T}=6.6 \text { hours }
$$

Note that Earth is in a superposition of quantum states and our [energy] scale is too low to disturb that superposition.

The fact that we can measure these rates [and anything else in the Solar System], with high precision and not disturbing the system, shows that, while uncertainties in measurement are fundamental, the size of uncertainty is a measurement problem arising from inadequate scale of observational energy, a relative quantity (Planck's constant, $\hbar$, as a dimensional constant between entangled properties, must be a relative, not absolute constant).

Note that this also shows the nature of superposition - as postulated by $\mathrm{CR}$, a system cannot be in multiple states at the same time unless these are separated in space, and cannot be in multiple states at the same space unless they are separated in time.

For Earth heart rate $=$ my rest heart rate $=76 \mathrm{bpm}:$

1 Earth scale minute $=76 * 6.6=495$ hours $=20.625$ days

Given the number of heartbeats $\mathrm{EH}_{3 / 3}\left(1 * 10^{9}, 4 * 10^{9}\right)=2 * 10^{9}$ and scale invariance of heartbeats, the period is:

$$
T_{x}=2 * 10^{9} * 6.6=1.32 * 10^{10} h=1.51 * 10^{6} \text { years }
$$

This number of heartbeats with a heart rate of $76 \mathrm{bpm}$ corresponds to a human lifespan of 50 years. This, I consider as the global average human lifespan over the course of evolution on Earth's surface (or at least, during the last 1.512 million years).

With such number of heart beats[30] (between incarnations), the Earth would belong to, not only mammalian species, but homo species.

The 3rd order cycle of the Solar System $\left(1.512 * 10^{6}\right.$ years $)$ can thus be interpreted as evidence of evolutionary entanglement - a man is in its path of 
evolution between the standard scale carbon atom and the Solar System (large scale carbon atom).

To species accustomed to the concept of birth and extroverted nature it might appear that Earth never fully develops.

This is most certainly not the case - life past the embryonic form to us always results in change of environment, but this is only due to inadequacy of current environment to ensure the continuity of progressive evolution, one which includes growth of the physical form.

Once extroverted intelligence evolves into, relatively more energy efficient, introverted intelligence, there is no need for physical growth or reason for most of conventional physical organs.

Spherical form may thus be interpreted as a pinnacle of evolution, rather than an undeveloped form of life, even though it externally manifests itself as a mere particle, or, a piece of rock. If a man should regard any cosmic phenomena as a deity, it should certainly be Earth, as it would be the one closest to us. A god with whom we are strongly entangled and thus evolutionary depend on. A god who can take and give, and thus be real.

\subsubsection{Speed of time}

Space/time may be represented by 3 dimensions, one positively polarized (space), one negative (time) and one neutral (event horizon in between).

These 3 dimensions are spatially separated and quantized, but they are entangled and may orbit the same body, such that orbital velocity of the event horizon is:

$$
v_{E H}=\left(v_{S}-v_{T}\right) * C,
$$

where $\mathrm{v}_{S}$ and $\mathrm{v}_{T}$ are orbital velocities of space and time dimensions, respectively. 3rd order space for Earth is 1-dimensional - the Earth is an inflated quantum of space/time orbiting the Sun. Dimensions of [3rd order] space and time of Earth have been further separated during inflation, but they remain entangled. Assuming that space dimension is [at] Earth's orbital radius, the time dimension should be somewhere in the higher orbit.

Time dimension velocity is quantized by $\mathrm{v}_{S}$ :

$$
\begin{gathered}
v_{T}(n)=\left\{(n+j)+(n-i) \pm[(n+j) *(n-i)]^{-1}\right\}^{-i} \\
*\left\{(n+j) \pm\left[(n+j) *(n-i)^{2}\right]^{-1}\right\}^{-j} * v_{S}(n) \\
n, i, j \in \mathbb{Z} \\
i=n-C_{1}, j=C_{2}-n, i+j=C_{2}-C_{1}
\end{gathered}
$$




$$
C_{1}, C_{2} \in \mathbb{N}
$$

The values in square brackets, depending on the sign, give maximum and minimum values of $\mathrm{v}_{T}$ during the cycle state. The average (mean) $\mathrm{v}_{T}$ :

$$
\begin{gathered}
v_{T}(n)_{A V G}=[(n+j)+(n-i)]^{-i} *[(n+j)]^{-j} * v_{S}(n)=(2 n+j-i)^{-i} *(n+j)^{-j} * v_{S}(n) \\
v_{T}(n)_{A V G}=\left(C_{1}+C_{2}\right)^{C_{1}-n} *\left(C_{2}\right)^{n-C_{2}} * v_{S}(n)
\end{gathered}
$$

For inner planets, in state $6 \mathrm{p} 4 \mathrm{n}$ :

$$
C_{1}=2, C_{2}=3
$$

$v_{T}(n)=\left[5+(3 * 2)^{-1}\right]^{-i} *\left[3+(3 * 4)^{-1}\right]^{-j} * v_{S}(n)=\left(5+6^{-1}\right)^{-i} *\left(3+12^{-1}\right)^{-j} * v_{S}(n)$

$$
\begin{gathered}
i=n-2, j=3-n, i+j=1 \\
v_{T}(n) \approx \frac{1}{v_{n}} * v_{S}(n), v_{n}=v_{n-1}+2^{n-2}, v_{0}=\left(\frac{2}{3}\right)^{-1}=\left(\frac{N}{P}\right)^{-1}
\end{gathered}
$$

Solar System may also be observed as a hydrogen-like atom, where space, time and event horizon dimensions have been split into 4 component vectors (levels).

The event horizon velocity (derived from $\mathrm{v}_{S}$ and $\mathrm{v}_{T}$ ), given the orbital energy level vectors for inner $\left(\mathrm{n}_{1}\right)$, outer $\left(\mathrm{n}_{2}\right)$ planets and the oscillatory vector $\mathrm{k}$ :

$$
\begin{gathered}
\boldsymbol{n}_{1}=\left[\begin{array}{c}
5 \\
3 \\
3 \\
10
\end{array}\right], \boldsymbol{n}_{2}=\left[\begin{array}{l}
1 \\
3 \\
5 \\
5
\end{array}\right], \boldsymbol{k}=\left[\begin{array}{c}
0 \\
3^{1} \\
3^{2} \\
5^{2}
\end{array}\right] \\
\boldsymbol{v}_{E H}=\left(\boldsymbol{v}_{S}-\boldsymbol{v}_{T}\right) *\left(\frac{\boldsymbol{n}_{1}+\boldsymbol{k} \oplus \boldsymbol{n}_{2}}{10^{1}}+\frac{\boldsymbol{k}}{10^{2}}\right),
\end{gathered}
$$

where $\oplus$ is the sign operator:

$$
\boldsymbol{a} \oplus \boldsymbol{b}=\left[\begin{array}{l}
-1^{a_{1}+1} * b_{1} \\
-1^{a_{2}+1} * b_{2} \\
-1^{a_{3}+1} * b_{3} \\
-1^{a_{4}+1} * b_{4}
\end{array}\right]
$$


Note that the ratio of sums of elements of $n_{2}$ and $n_{1}$ is:

$$
\frac{\sum n_{2}}{\sum n_{1}}=\frac{14}{21}=\frac{2}{3}=\frac{N}{P}
$$

The event horizon velocity (from $\mathrm{v}_{S}$ only):

$$
\begin{gathered}
v_{E H_{0}}(n)=\frac{r_{S}(n)}{r_{M a r s}} v_{S}(n)=\frac{1}{r_{M a r s}} \sqrt{G M * r_{S}(n)} \\
c_{E H}=1 \frac{k m}{s} \\
v_{E H}(n)=v_{E H_{0}}(n)+(-1)^{\left(\delta_{j n, 2}\right)}\left[1+2^{\left(1-\delta_{j, i+1}\right)}-(i j+1) 3^{\left(-2^{\delta_{j, i+1}}\right) \frac{1}{2}}\right] c_{E H},
\end{gathered}
$$

\begin{tabular}{|c|c|c|c|c|c|c|c|c|c|}
\hline $\mathrm{n}$ & Planet & $\mathrm{i}$ & $\mathrm{j}$ & $\begin{array}{l}\mathrm{v}_{S} \\
(\mathrm{~km} / \mathrm{s})\end{array}$ & $\begin{array}{l}\mathrm{v}_{T} \mathrm{~km} / \mathrm{s} \\
\text { (entan- } \\
\text { glement) }\end{array}$ & $\begin{array}{l}\sigma_{T} \text { (current } \\
\text { value) }\end{array}$ & $\begin{array}{l}\mathrm{v}_{E H_{0}} \\
\text { (entan- } \\
\text { glement) } \\
\mathrm{km} / \mathrm{s}\end{array}$ & $\begin{array}{l}\sigma_{E H_{0}} \\
\text { (neu- } \\
\text { tron } \\
\text { correc- } \\
\text { tion) }\end{array}$ & $\begin{array}{l}\mathrm{v}_{E H} \\
\text { (entan- } \\
\text { glement) } \\
\mathrm{km} / \mathrm{s}\end{array}$ \\
\hline 4 & Mercury & 2 & -1 & 47.36 & $\begin{array}{l}5.47 \\
\text { (Nep- } \\
\text { tune) }\end{array}$ & $\begin{array}{l}-2^{2} * 10^{-2} \\
=-0.04\end{array}$ & $\begin{array}{l}12.033 \\
\text { (Jupiter) }\end{array}$ & +4.73 & $\begin{array}{l}16.77 \\
\text { (Hygiea) }\end{array}$ \\
\hline 3 & Venus & 1 & 0 & 35.02 & $\begin{array}{l}6.78 \\
\text { (Uranus) }\end{array}$ & $\begin{array}{l}+2^{1} * 10^{-2} \\
=+0.02\end{array}$ & $\begin{array}{l}16.63 \\
\text { (Hygiea) }\end{array}$ & +1.275 & $\begin{array}{l}17.9 \\
\text { (Ceres) }\end{array}$ \\
\hline 2 & Earth & 0 & 1 & 29.78 & $\begin{array}{l}9.66 \\
\text { (Saturn) }\end{array}$ & $\begin{array}{l}+2^{1} * 10^{-2} \\
=+0.02\end{array}$ & $\begin{array}{l}19.55 \\
\text { (Vesta) }\end{array}$ & -1.66 & $\begin{array}{l}17.88 \\
\text { (Pallas) }\end{array}$ \\
\hline 1 & Mars & -1 & 2 & 24.07 & $\begin{array}{l}13.08 \\
\text { (Jupiter) }\end{array}$ & $\begin{array}{l}-2^{1} * 10^{-2} \\
=-0.02\end{array}$ & $\begin{array}{l}24.07 \\
\text { (Mars) }\end{array}$ & -4.73 & $\begin{array}{l}19.34 \\
\text { (Vesta) }\end{array}$ \\
\hline
\end{tabular}

where $\delta_{a, b}$ is the Kronecker delta function. Table 17 shows space velocities for

Table 17: Orbital velocities of time and event horizon dimensions

inner planets and calculated velocities of time and event horizon dimensions along with their correlation with bodies of the Solar System.

Evidently, the speed of time dimension decreases as the speed of space increases and orbits are quantized and entangled (as predicted by CR):

$$
\frac{v_{S}}{v_{T}}=\sqrt{\frac{r_{T}}{r_{S}}} \approx\left(C_{1}+C_{2}\right)^{n-C_{1}} * C_{2}^{C_{2}-n}
$$

Orbital velocity of Earth's space is $29.78 \mathrm{~km} / \mathrm{s}$. Average velocity of the event horizon for Earth is $2 / 3$ of this velocity, while the average velocity of time dimension is $1 / 3$ of this velocity:

$$
v_{E H_{A V G}}=\frac{2}{3} 29.78=19.85333^{\prime} \mathrm{km} / \mathrm{s}
$$




$$
v_{T_{A V G}}=c_{t_{1}}=\frac{1}{3} 29.78=9.92666^{\prime} \mathrm{km} / \mathrm{s}
$$

Orbital radius of the time dimension is the space dimension of Saturn - Earth's time dimension is entangled with the space dimension of Saturn (time dimension of Saturn is entangled with Earth space dimension).

Average event horizon is entangled with the current orbit of Vesta, the dwarf planet.

Deviation of $\mathrm{v}_{T_{A V G}}$ from current Saturn orbit is equal to deviation of $\mathrm{v}_{E H_{A V G}}$ from current Vesta:

$$
v_{V e s t a}=\frac{v_{\text {Saturn }}}{v_{T_{A V G}}} * v_{E H_{A V G}}=3 * \frac{9.68}{29.78} * \frac{2}{3} 29.78=9.68 * 2=19.36 \mathrm{~km} / \mathrm{s}
$$

Speed of time for human bodies $\left(c_{t_{0}}\right)$ is equal to standard speed of light $c$, given the average life expectancy of 50 years $\left(2^{*} 10^{9}\right.$ heartbeats with $76 \mathrm{bpm}$ heart rate), the 3rd order period of Earth's existence cycle is:

$$
T_{x}=\frac{c_{t_{0}}}{c_{t_{1}}} * 50 \text { years }=3 * \frac{2.99792458 * 10^{8}}{29.78 * 10^{3}} * 50 \text { years }=1.51 * 10^{6} \text { years }
$$

\subsection{Body mass}

I have previously calculated initial (formative) real mass of Earth[31] (as a particle) to be $2.93676 * 10^{19} \mathrm{~kg}$.

If Earth is a living organism, predicting real mass of Earth in the same way as it is done with other organisms should give the result of the same order of magnitude (it likely won't be of equal value as Earth is evolving, gaining and loosing mass in the process).

Assuming that Earth is a mammal (or evolved from mammal), given the 3rd order existence half-life (period) $\mathrm{T}_{x}$ of $1.512 * 10^{6}$ years, mass can be calculated from empirical relationship between mass and lifespan of mammalian species.

$$
\left(\frac{m_{E}}{m}\right)^{\frac{1}{4}} * T_{x_{M}}=T_{x}
$$

Given human adult mass $\mathrm{m}$ of $84 \mathrm{~kg}$ and lifespan $\mathrm{T}_{x_{M}}$ of 50 years, mass of earth $\mathrm{m}_{E}$ is:

$$
\begin{aligned}
& m_{E}=m\left(\frac{T_{x}}{T_{x_{M}}}\right)^{4} \\
& m_{E}=7 * 10^{19} \mathrm{~kg}
\end{aligned}
$$


Note that the value of $\mathrm{T}_{x}^{4}, 5.2 * 10^{24}$ is roughly the value of the total gravitational mass of Earth $\left(\mathrm{M}=5.9723 * 10^{24}\right)$.

The result can be further verified by the previously established (in CR) equation for real mass:

$$
m_{E}=m_{r e}=\left(1-\sqrt{1-\frac{v_{r e}^{2}}{c_{s}^{2}}}\right) m_{i m g}
$$

where

$$
\begin{gathered}
v_{r e}=\frac{2 \pi r_{r e}}{T_{r e}}=\frac{2 \pi r_{s}}{T_{r e}} \\
c_{s}=\sqrt{\frac{G m_{i m g}}{r_{s}}} \approx \sqrt{\frac{G M}{r_{s}}}
\end{gathered}
$$

Using $\mathrm{T}_{r e}=23.9 * 60 * 60=86040 \mathrm{~s}, \mathrm{G}=\mathrm{G}_{0}=6.673899 * 10^{-11} \mathrm{~m}^{3} / \mathrm{kgs}^{2}, \mathrm{r}_{s}=$ $1206115 \mathrm{~m}, \mathrm{~m}_{\text {img }} \approx \mathrm{M}=5.9723 * 10^{24} \mathrm{~kg}$ :

$$
m_{E}=7 * 10^{19} \mathrm{~kg}
$$

The results agree and show that Earth has gained roughly double the mass it had at the point of inflation/deflation of the gravitational maximum.

One might argue that it is impossible for Earth to have such low mass, as there is $10^{18} \mathrm{~kg}$ in surface oceans alone, $10^{22} \mathrm{~kg}$ in the crust, $10^{23} \mathrm{~kg}$ in the inner core and more in the mantle (based on density inferred from seismic profiles), however, these values are relative to the gravitational constant of the standard $\left(\mathrm{U}_{0}\right)$ scale $\mathrm{G}_{0}\left(6.674 * 10^{-11} \mathrm{~m}^{3} / \mathrm{kgs}^{2}\right)$.

As stated before, proper relativistic (effective) mass of Earth on $U_{1}$ scale is relative to $\mathrm{G}_{1}\left(5.731534632 * 10^{-6} \mathrm{~m}^{3} / \mathrm{kgs}^{2}\right)$.

Proper, relativistic, equation for relationship between mass and lifespan is thus:

$$
G_{1} m_{E}=G_{0} m\left(\frac{T_{x}}{T_{x_{M}}}\right)^{4}
$$

Various results can now be obtained, depending on the value of variables, as shown in Table 18.

Note, however, that obtained mass can also be interpreted as current imaginary mass, rather than real mass (in that case, real mass $\left.=5.9723 * 10^{24} \mathrm{~kg}-7 * 10^{19} \mathrm{~kg} \approx 5.9723 * 10^{24} \mathrm{~kg}\right)-$ as the gravitational well of a maximum acquires mass, this mass is shielding (replacing) the gravity of the maximum, so the obtained mass represents the remaining capacity for real mass (current img part of total gravitational mass), rather than current real mass. 


\begin{tabular}{c|c|c|c|r}
$\mathrm{n}$ & $\mathrm{G}_{1}\left[\mathrm{~m}^{3} / \mathrm{kgs}^{2}\right]$ & $\mathrm{G}_{0}\left[\mathrm{~m}^{3} / \mathrm{kgs}^{2}\right]$ & $\mathrm{T}_{x}$ & $\mathrm{~m}_{E}(\mathrm{n})[\mathrm{kg}]$ \\
\hline 1 & $5.731534632 * 10^{-6}$ & $6.674^{*} 10^{-11}$ & $25.82 \mathrm{My}$ & $6.9543 * 10^{19}$ \\
2 & $6.674 * 10^{-11}$ & $6.674 * 10^{-11}$ & $1.512 \mathrm{My}$ & $7.0244 * 10^{19}$ \\
3 & $6.674 * 10^{-11}$ & $6.674 * 10^{-11}$ & $25.82 \mathrm{My}$ & $5.9723 * 10^{24}$ \\
4 & $6.674 * 10^{-11}$ & $5.731534632 * 10^{-6}$ & $1.512 \mathrm{My}$ & $7.1816 * 10^{22}$ \\
5 & $6.674 * 10^{-11}$ & $5.731534632 * 10^{-6}$ & $25.82 \mathrm{My}$ & $5.1290 * 10^{29}$ \\
6 & $6.674 * 10^{-11}$ & $6.674 * 10^{-11}$ & $19.3 \mathrm{~s}$ & $1.8802 * 10^{-30}$ \\
7 & $4.9000394^{*} 10^{-2}$ & $6.674 * 10^{-11}$ & $4.25 \mathrm{~Gy}$ & $5.9723 * 10^{24}$
\end{tabular}

Table 18: Relative Earth mass

Here, $\mathrm{m}_{E}(1)$ is the proper relativistic mass of Earth calculated with 2nd order $\mathrm{T}_{x}, \mathrm{~m}_{E}(2)$ is the proper relativistic mass calculated using $3 \mathrm{rd}$ order $\mathrm{T}_{x}$. Third mass, $\mathrm{m}_{E}(3)$, is the mass of Earth relative to standard scale $\left(\mathrm{m}_{E_{0}}\right)$ calculated using 2 nd order $\mathrm{T}_{x}$.

Masses $\mathrm{m}_{E}(4)$ and $\mathrm{m}_{E}(5)$ could be considered as inverse (or anti) masses of Earth relative to its [past] event horizon (inner core maximum).

Note that $\mathrm{m}_{E}(4)$ is [roughly?] equal to $2 / 3$ of the mass of the Earth's inner core, while $\mathrm{m}_{E}(5)$ is roughly $1 / 4$ of the Sun's mass.

Note also the presence of multiple periods in the cycling of Earth's [maximum] existence, 1.512 My and 25.82 My. While the shorter period could be considered as a fossil of the Solar System $\mathrm{U}_{0}$ half-life $\left({ }^{10} \mathrm{Be}_{0}\right)$, this entanglement cannot be lost completely and some time compression at the end of $1.512 \mathrm{My}$ cycles can also be expected.

While the periods of 2nd and 3rd order represent the half-life of Earth's gravitational maximum quanta, these do not represent the lifespan of Earth.

At the end of these cycles, major maximum, effectively, only temporarily collapses (partially, in time and space), proportionally to the cycle period. If the maximum is interpreted as a soul, which I am convinced is the correct interpretation, such collapse is, effectively, a temporary loss of consciousness. I have previously hypothesized that the Solar System is a product of annihilation and inflation of ${ }^{10} \mathrm{C}$ and ${ }^{10} \mathrm{Be}$ atoms of smaller scale, thus, entanglement with ${ }^{10} \mathrm{C}$ can also be expected, although the collapse and the induced time (evolution) compression should be negligible due to short half-life (19.3 s) of ${ }^{10} \mathrm{C}$.

Note that Earth is in 2e configuration, and with $\mathrm{T}_{x}$ of $19.3 \mathrm{~s}$, mass of Earth $\left[\mathrm{m}_{E}(6)\right]$ becomes roughly equal to the mass of 2 standard electrons (or positrons).

Also note that the initial real mass of Earth $\left(2.93676 * 10^{19} \mathrm{~kg}\right)$ is roughly half of $\mathrm{m}_{E}(1)$ and should correspond to 1e configuration.

If $\mathrm{m}_{E}(4)$ and $\mathrm{m}_{E}(5)$ are correlated with Earth's inner core and Sun mass, the data suggests asymmetry between mass and inverse mass, growing with period $\mathrm{T}_{x}$.

The solution is the inflation of $\mathrm{T}_{x}$ and/or $\mathrm{G}$.

With $\mathrm{G}_{0}$ [roughly] equal to $2.222 * 10^{-5} \mathrm{~m}^{3} / \mathrm{kgs}^{2}, \mathrm{~m}_{E}(5)$ becomes equal to the mass of the Sun, while for $\mathrm{G}_{0}$ [roughly] equal to $1.9561 * 10^{-5}, \mathrm{~m}_{E}(4)$ becomes equal to to the proper relativistic mass of the Sun.

The same can be obtained with $\mathrm{T}_{x}$ equal to $36.23 \mathrm{My}$ and 2.06 $\mathrm{My}$, respectively. 
With a period of 555619.11 years, $\mathrm{m}_{E}(4)$ becomes equal to inner core mass (assuming that mass is $1.1 * 10^{23} \mathrm{~kg}$ ).

Interestingly, for $\mathrm{T}_{x}$ equal to the 1st order period $(4.25 \mathrm{~Gy})$, the result of equation M1.1, rounded to 2 decimals, is equal to speed of light on $\mathrm{U}_{1}$ scale $\left(2.93 * 10^{6} \mathrm{~m} / \mathrm{s}\right)$ multiplied by $10^{17}$.

Note also that the ratio between $\mathrm{G}_{1}(7)$ and $\mathrm{G}_{1}(1)$ is roughly equal to ratio between $\mathrm{G}_{1}(1)$ and $\mathrm{G}_{0}(1)$ divided by 10 :

$$
G_{1}(7) \approx \frac{1}{10} \frac{G_{1}(1)}{G_{0}(1)} G_{1}(1)
$$

which is consistent with association of different G's to different vertical energy levels and therefor to scale (period) of general oscillation.

If $\mathrm{G}_{0}(1)$ would, as hypothesized previously, belong to $\mathrm{U}_{0}$ scale, G1(1) should be associated with $\mathrm{U}_{1}$ scale and $\mathrm{G1}(7)$ with $\mathrm{U}_{2}$ scale.

If one assumes that:

$$
G_{1}(7)=\frac{1}{10} \frac{G_{1}(1)}{G_{0}(1)} G_{1}(1)
$$

one obtains a $\mathrm{T}_{x}$ of the 1 st order of 4.254788 Gy $\left(4.254788 * 10^{9}\right.$ years $)$.

\subsection{Future development, neurogenesis}

Here I hypothesize that cultivation of life on the surface of a planet is a cultivation of precursor neuron cells and proteins (relative to the planet) which are, at the point of differentiation transferred to planet's [brain] mantle layers. Similar to accelerated (time compressed) evolution during human embryo-genesis, I hypothesize that effective time compression occurs during planetary evolution too - with the end of each cycle of general oscillation of the Solar System (Earth) and with amount of compression being inversely proportional to cycle order.

The points of differentiation and migration in neurogenesis are major mass extinction events (although limited transfer might occur in smaller extinctions too), which are thus only relative extinctions - life is not completely extinct, it undergoes rapid evolution and migrates away to mantle where it continues evolution.

I hypothesize that Earth's brain has, like human brain, 6 major layers, and that complete formation of these layers requires 6 major mass extinctions during Phanerozoic.

At this point, there is no doubt that we are amidst an major extinction event, a 6 th one.

Being part of neurogenesis, extinction events must be programmed at some level and, at least roughly, periodic.

Extinction events have relative triggers. While in the past these may have been impactors and volcanism, current extinction seems to have an anthropogenic trigger. 
Thus, one might conclude that current extinction is not part of neurogenesis, rather a part of unlimited cancer growth. However, tumors in humans are known to induce neurogenesis (it is one mechanism enabling migration - metastasis).

I find the induction questionable though - humans are not consciously triggering neurogenesis on Earth, it is thus more plausible for neurogenesis to be a reaction of the immune system to inhibit cancer growth. Extinctions coupled with neurogenesis go in favor of such hypothesis. In case of cancer in humans though, and at least during adult neurogenesis in humans, the immune system seems to fail to cure or exterminate the cancerous cells in most cases (in case of humans who are cancerous themselves for Earth, I believe).

The immune system of Earth though, should be more advanced, and I believe cancerous homo.beta will be subdued.

Homo.beta refers to species of humans currently inhabiting the Earth's surface, self-proclaimed homo sapiens. For various reasons, I consider the title homo sapiens premature for this species, so I have reserved it for an evolved form of human.

Judging by past major extinctions, and correlating with human neurogenesis, these events should be expected with the advancement of planetary neurogenesis:

- increasing rate of volcanism and earthquakes (due to gyrification/formation of brain tissue, curing cancer?),

- asteroid/cometary impacts (providing energy, acting as specific event triggers, curing cancer?),

- sea level changes (melting of polar ice to enable migration, curing cancer?).

Migration of cells and proteins from surface to mantle layers requires tunnels connecting these regions. Most likely, these tunnels exist on specific places and are recreated or reopened at time of migration. A likely place for such tunnel opening on surface is the south pole, but may exist on north pole of a planet too.

One fact going in favor of this hypothesis is that during all previous major extinctions there were periods when poles were free from ice. Although, one could argue that, during Phanerozoic, world was more often without polar ice caps, than with.

Cells and proteins are transferred with the flow of cerebrospinal fluid (CSF) a salty ocean. In humans, CSF has a $\mathrm{pH}$ of 7.33, and, since $\mathrm{pH}$ is scale invariant, I believe the current acidification of Earth's oceans will continue until pH drops 
to this value, when migration should follow. Afterwards, new surface water may be delivered by asteroid (cometary) impacts, but it is also possible that some or most of it returns from the mantle.

Based on correlation with atmospheric $\mathrm{CO}_{2}$, climate models predict the hypothesized $\mathrm{pH}$ minimum in year $2300 \mathrm{AD}$ for an atmospheric concentration of $\mathrm{CO}_{2}$ of 1900 ppmv[32] (all fossil-fuel sources burned).

Note that the $\mathrm{pH}$ minimum (7.33 as hypothesized), associated with CSF, must have been reached before on Earth during mass extinction events. The research confirms this, ie. for Permo-Triassic[33].

The cited work shows a [relatively] rapid drop in $\mathrm{pH}$ to a minimum, followed by rapid increase and slow progress toward stabilization. Two models were developed for $\mathrm{CO}_{2} / \mathrm{pH}$ concentration (low- and high- $\mathrm{CO}_{2}$, with a difference in $\mathrm{pH}$ minimum between the two being less than 0.2 ), in high- $\mathrm{CO}_{2}$ model, the $\mathrm{pH}$ minimum is 7.35 , very close to predicted 7.33 minimum. The work, however, favors the low- $\mathrm{CO}_{2}$ model, so it cannot be excluded that Earth's CSF pH is somewhat higher (less acidic) than human.

In any case, the existence of a $\mathrm{pH}$ minimum and it's value strongly support the theory of neurogenesis.

A precursor of 6 mantle layers has likely been created in events during Precambrian era, while population with neuron cells and final formation is occurring in Phanerozoic.

There have been 5 major extinctions in Phanerozoic, thus the next event should populate top layers and complete the formation of the final layer (I):

Formed layers of Earth's brain are shown on Fig. 17. Comparing with other layers, it seems evident that layer I is yet to be completed - green line shows possible seismic velocities after formation.

Energy from the Sun provides incubation energy used for the maintenance of the Earth's surface ecosystem and weak evolution, but additional energy is needed for the formation of brain layers of homo.omega.

Here, homo.omega is a species of life Earth belongs to.

This energy is delivered through asteroid (food) and cometary (water) impacts.

Year $2300 \mathrm{AD}$ for the event is very conservative though, as it is based on linear extrapolation, does not include rising water temperatures and reaction of the biosphere.

Acidification of water at these events must be, in large part, driven by injections of gases through oceanic ridges and vents which would introduce significant 


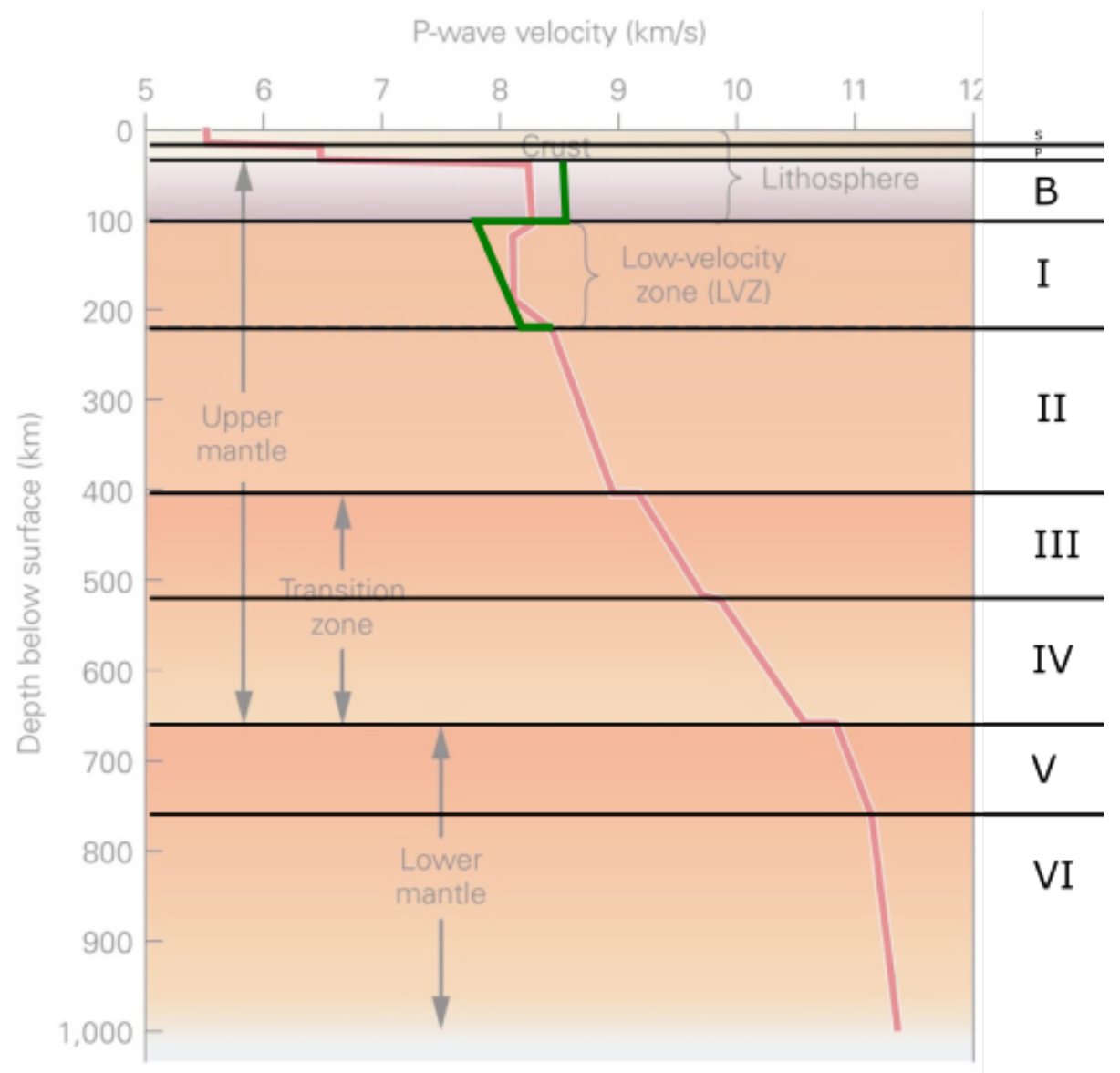

(a) The velocity of P-waves changes with depth in the mantle.

Figure 17: Layers of Earth's brain, superimposed on seismic velocities ${ }^{34}$ 
departure from linear correlation of $\mathrm{pH}$ with atmospheric $\mathrm{CO}_{2}$.

Mathematical analysis of past extinctions[35] also suggests sooner triggering of 6th major extinction event, by the year 2100[36].

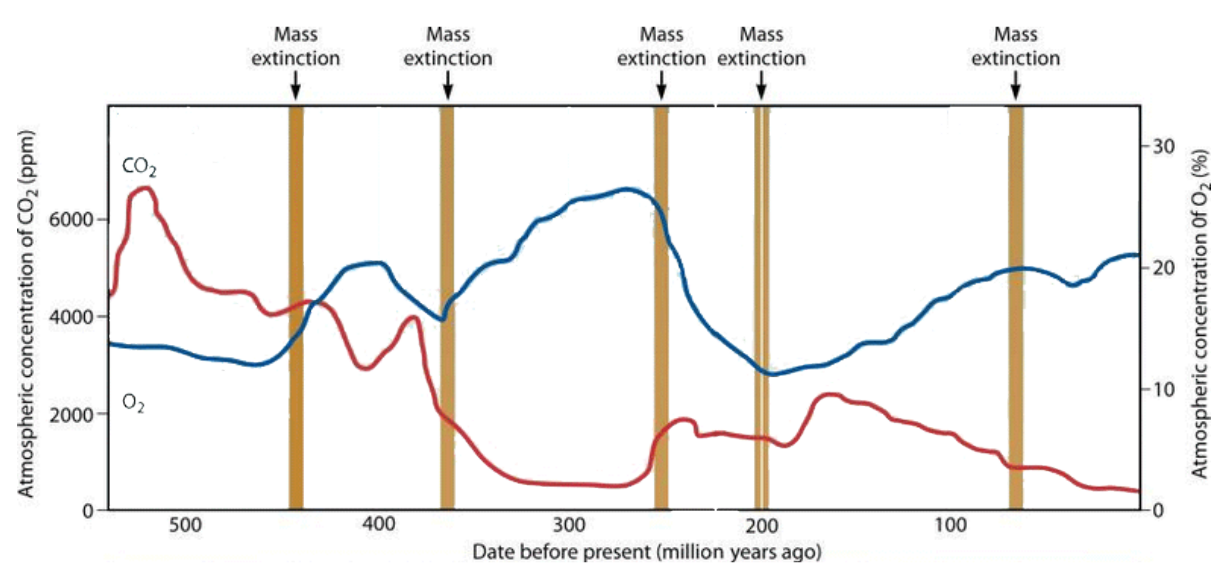

Figure 18: The history of atmospheric $\mathrm{CO}_{2}$ concentration ${ }^{37}$

From Fig. 18 and more recent models[38], it is evident that $\mathrm{CO}_{2}$ concentration has a decreasing trend (expected due to increased energy from the Sun = less greenhouse gases needed to maintain the temperature).

Everything in nature oscillates (and fluctuates) so this decrease in amplitude should not be linear either, however some periodicity in extinctions must be present.

Statistically significant periodicity of extinctions[39] (at least in the last 250 million years) has been noted before - 26, and more recently, 27 million years between extinctions[40]. In any case, due to differences in extinction strength, multiple harmonics (or energy splitting of a single oscillator) are possible.

Using available data, one can construct models for atmospheric $\mathrm{CO}_{2}$ concentration synchronized with the oceanic $\mathrm{pH}$ minimum of a particular major extinction, as shown in Table 19.

\begin{tabular}{l|l|l|l|l|l} 
year [mya] & $\begin{array}{l}\text { a) } \mathrm{CO}_{2} \\
{[\mathrm{ppm}]}\end{array}$ & $\begin{array}{l}\text { b) } \mathrm{CO}_{2} \\
{[\mathrm{ppm}]}\end{array}$ & $\begin{array}{l}\text { c) } \mathrm{CO}_{2} \\
{[\mathrm{ppm}]}\end{array}$ & $\begin{array}{l}\text { d) } \mathrm{CO}_{2} \\
{[\mathrm{ppm}]}\end{array}$ & $\begin{array}{l}\text { e) } \quad \mathrm{CO}_{2} \\
{[\mathrm{ppm}]}\end{array}$ \\
\hline 444 & 3800 & 200 & 2000 & 3800 & 2000 \\
370 & 1000 & 2000 & 1000 & 1800 & 1200 \\
252 & 800 & 900 & 800 & 800 & 800 \\
201 & 1800 & 1800 & 1800 & 1800 & 600 \\
66 & 250 & 250 & 250 & 300 & 500 \\
0 & 450 & 700 & 750 & 800 & 450
\end{tabular}

Table 19: $\mathrm{CO}_{2} \mathrm{pH}$ minimum marker models 
a)

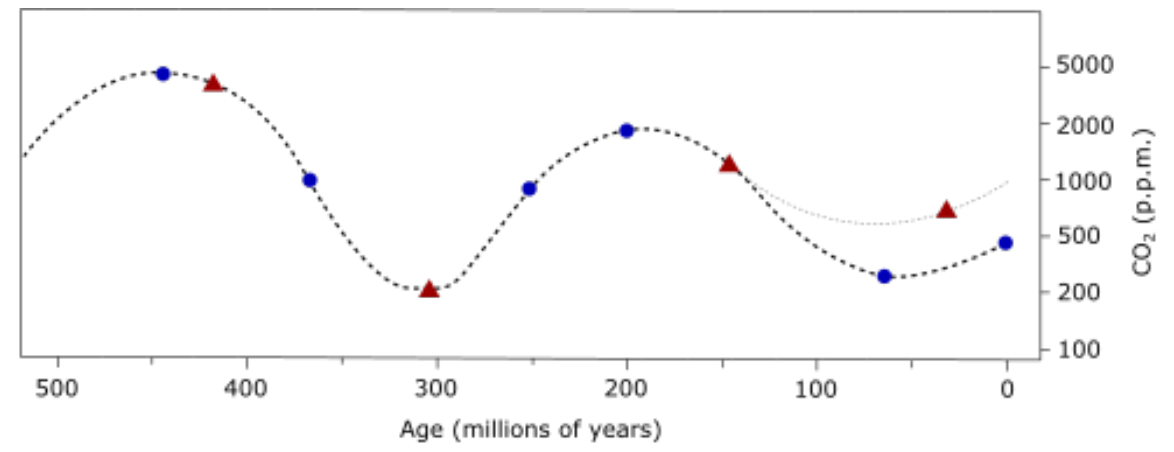

b)

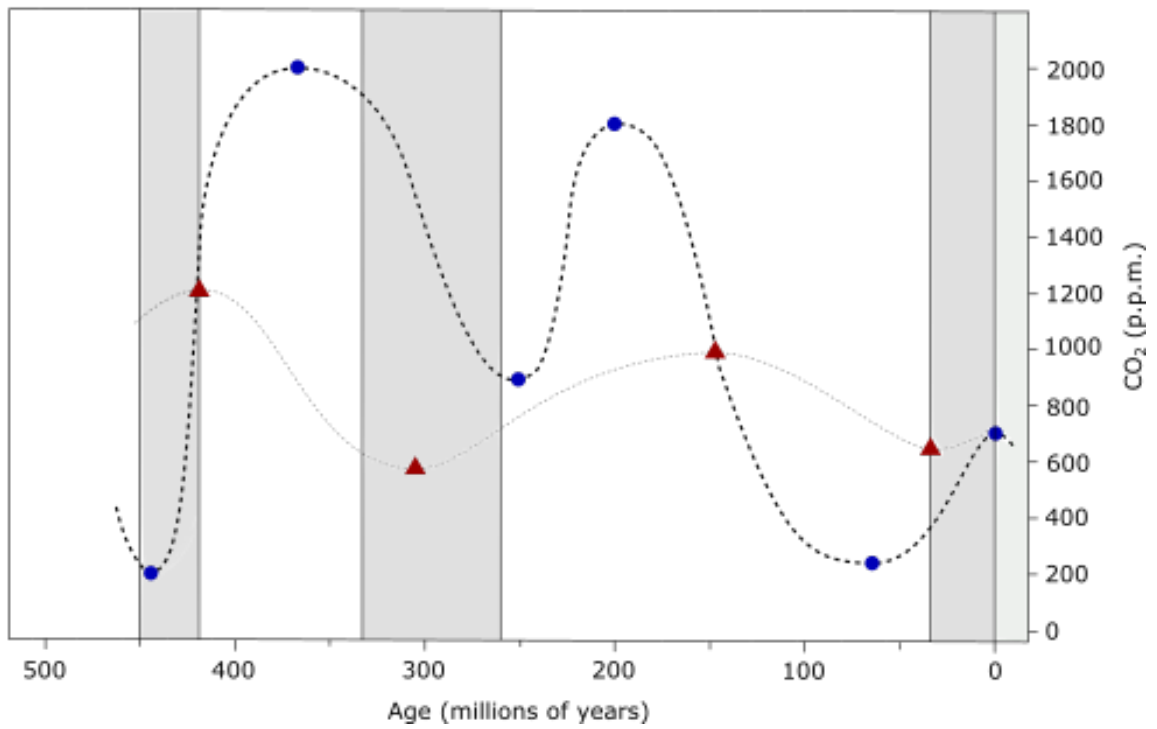

Figure 19: $\mathrm{CO}_{2} \mathrm{pH}$ minimum marker models a) and b) (blue dots = major extinction events, red $=$ minor extinction events, on b) grey = icehouse periods, white $=$ greenhouse periods) 
Models are constructed in such a way to simulate oscillation of $\mathrm{CO}_{2}$ markers and compression of the amplitude with time, but they are also quantized - each marker is a multiple of $50 \mathrm{ppm} \mathrm{CO}_{2}$ quantum.

Some of the models are shown on Fig. 19, blue dots are major extinction events, red triangles are minor extinction events (the curve does not necessarily follow actual $\mathrm{CO}_{2}$ levels between the extinctions, it is only used to illustrate oscillation of markers).

From these models, grouping of extinctions (suggesting oscillation of frequency) becomes more apparent. Major extinctions can be grouped into pairs separated by $126.5( \pm 8.5)$ million years, while paired extinctions are separated by roughly half that distance $-62.5( \pm 11.5)$ million years. Minor extinctions (420, 305, 145 and 34 mya) may be grouped in the same way - pairs separated by 160 million years, $113( \pm 2)$ million years separation of paired extinctions.

Model a) is the product of energy level splitting of a single oscillator, while b) is the product of 2 harmonic oscillators - one high energy (major) and one low energy (minor).

Points on the curve should not be interpreted as maximum atmospheric $\mathrm{CO}_{2}$ levels across the boundary, simply the points of migration or $\mathrm{pH}$ minimums.

While these particular models are highly speculative, all Phanerozoic $\mathrm{CO}_{2}$ models show decreasing $\mathrm{CO}_{2}$ over time (this should be more evident when comparing boundaries of major extinction events) and recent research shows that maximum atmospheric $\mathrm{CO}_{2}$ across the $\mathrm{K}-\mathrm{Pg}$ boundary (last major extinction) was $875 \mathrm{ppm}[41]$.

Thus, the maximum atmospheric $\mathrm{CO}_{2}$ concentration during current extinction should be lower than $875 \mathrm{ppm}$, probably not higher than $800 \mathrm{ppm}$ and perhaps even lower (suggesting that a larger part of acidification will not be sourced in atmospheric $\mathrm{CO}_{2}$ ).

Figure 20: Recent history of $\mathrm{CO}_{2}$ concentration ${ }^{42}$

Recent history of $\mathrm{CO}_{2}$ concentration is shown on Fig. 20. Assuming that $\mathrm{CO}_{2}$ has been, during that history, correlated with rate of evolution, one can extrapolate the relation for accelerated evolution of the current extinction.

Extrapolating from Fig. 20, from year 1850 onward:

$$
\begin{aligned}
C O_{2} & =300 *\left(\frac{6}{5} * 2^{45 x^{2}}\right)^{x} \mathrm{ppmv} \\
x & =\frac{T-1905}{10 * 55}=\frac{T-1905}{550}
\end{aligned}
$$

which, for the concentration of 800 ppmv gives year $\mathrm{T}=2075$.

Note that the equation roughly corresponds to IPCC RCP8.5 (Representative Concentration Pathway 8.5) scenario. Both predict equal $\mathrm{CO}_{2}$ 
for year 2100 , however, RCP 8.5 predicts $800 \mathrm{ppm}$ to be reached sooner - in year 2066.

RCP8.5 is considered a worst-case scenario and, at this point, also considered highly unlikely, as coal is increasingly being replaced by renewable energy sources.

However, while replacement of coal with other energy sources may affect human $\mathrm{CO}_{2}$ emissions, it is not reducing human impact on nature, which is not proportional to $\mathrm{CO}_{2}$ emissions, rather to energy (resources) consumption, which is growing as usual.

If the point of no return is passed, human emissions are completely irrelevant and positive feedback mechanisms will produce climate consistent with the RCP 8.5 scenario.

Humanity may be slowly abandoning the business of $\mathrm{CO}_{2}$ emissions, but, as proper cancer, it has not abandoned the unsustainable infinite growth policy.

Climate is a part of an eco-system, it evolves with the eco-system, and one cannot expect that disruption of eco-systems won't impact climate. Mass extinctions are always synchronized with climate disruptions.

While humans may eventually reduce their $\mathrm{CO}_{2}$ emissions significantly, the rate of evolution should keep accelerating according to equation and, regardless of atmospheric $\mathrm{CO}_{2}$ (which may still be increasing even with 0 human emissions), the required $\mathrm{pH}$ minimum will eventually be reached.

Asteroid impacts should be happening before the migration, and increasing in frequency and energy overall. Although required energy might be lower than in previous major extinctions, it should still be significant.

Assuming impacts are quantized proportionally to a $50 \mathrm{ppm}$ quantum of $\mathrm{CO}_{2}$, given the $\mathrm{C} 1.1$ equation, one can calculate years of impact, as shown in Table 20. Evidently, there are good candidates among extinction causing asteroids in NEO (near Earth orbit) for calculated dates.

Fission of extinction pulses, and thus multiple impacts are plausible (ie. one in 2029 and other in 2066) - due to splitting of energy levels (breaking of Apophis - homo induced?), as has most likely happened in previous extinctions.

Note 1: According to current models based on Newtonian mechanics, none of these asteroids is on a collision course with Earth in near future. However, these models do not predict periodic existence/extinction pulses coupling with a collapse and inflation of gravitational maxima. Ejecta from the Sun can also alter the course of an asteroid, as well as passing of the Solar System through extrasolar asteroid field.

Since these impacts are DNA coded, they should not be questionable, it is only the source and method of delivery that may be unknown prior to 


\begin{tabular}{|c|c|c|c|c|c|}
\hline model & $\begin{array}{l}\mathrm{CO}_{2} \\
{[\mathrm{ppm}]}\end{array}$ & $\begin{array}{l}\text { year of } \\
\text { impact }\end{array}$ & $\begin{array}{l}\text { associated im- } \\
\text { pactor (diameter) }\end{array}$ & $\begin{array}{l}\text { impactor } \\
\text { closest } \\
\text { approaches }\end{array}$ & $\begin{array}{l}\text { 2nd order } \\
\text { impactor } \\
\text { (diameter) }\end{array}$ \\
\hline a), e) & 450 & 2029 & $\begin{array}{l}99942 \quad \text { Apophis } \\
(\approx 375 \mathrm{~m})\end{array}$ & 2029,2065 & \\
\hline b) & 700 & 2066 & $\begin{array}{l}99942 \quad \text { Apophis } \\
(\approx 375 \mathrm{~m})\end{array}$ & 2029,2065 & \\
\hline c) & 750 & 2071 & $\begin{array}{l}1866 \text { Sisyphus }(\approx 7 \\
\mathrm{km})\end{array}$ & 2071 & $\begin{array}{l}2000 \mathrm{SG}_{344} \\
(37 \mathrm{~m})\end{array}$ \\
\hline d) & 800 & 2075 & $\begin{array}{l}162173 \text { Ryugu }(\approx 1 \\
\mathrm{km})\end{array}$ & 2076 & \\
\hline
\end{tabular}

Table 20: Calculated impact dates and possible impactors $(2$ nd order $=$ lower energy)

the event.

Note 2: Interestingly, there was an impact event on Earth at the time when $400 \mathrm{ppm} \mathrm{CO}_{2}$ was reached (Chelyabinsk meteor, $\approx 20 \mathrm{~m}$ diameter, 2013.), agreeing with hypothesized $50 \mathrm{ppm}$ quantization.

However, even though the Chelyabinsk event occurred in the year of 400 ppm, I believe the events should be correlated with the average ppm value given by the $\mathrm{C} 1.1$ equation.

For $400 \mathrm{ppm}$, the equation gives year 2015 (one may thus assume the events occur at $\mathrm{T} \pm \mathrm{n}$ years, where $\mathrm{n}$ is time dependent, ie. decreasing by half with every $100 \mathrm{ppm}$ - being equal to 4 at $300 \mathrm{ppm}, 2$ at 400 ppm, etc.). Assuming probability of correlation of these events with $\mathrm{CO}_{2}$ significantly increases once $\mathrm{CO}_{2}$ rises above background levels, the first event should have occurred at $300 \mathrm{ppm}$ - the beginning of industrial revolution. Indeed, one such event has [almost?] occurred at 300 ppm Tunguska, 1908.

The equation gives year 1992 for $350 \mathrm{ppm}$. No meteors of comparable impact energy were recorded in or around 1992., probably eliminating significant direct impacts on land area. If such event did occur, it has likely occurred over the ocean (or island), triggering large waves and possibly earthquakes. Interestingly, an 7.2+ magnitude earthquake and tsunami wave did occur offshore in Nicaragua - in 1992. This earthquake is notable for tsunami wave being unusually large $(9.9 \mathrm{~m}$ high) for the strength of the earthquake (belonging to a group of rare tsunami earthquakes).

I do not believe, however, that the impact caused the earthquake. This was likely the effect of synchronization of events (synchronicity) - the tsunami was caused by the earthquake but it was amplified by the impact. The Earth is a living being and it would not be surprising it reacts 
to incoming bolides and impactors (just like humans do).

I have witnessed such synchronization myself - on 2019.03.07 I have observed a larger meteor burning up in the atmosphere exactly at the time of earthquake in Hungary, it's trajectory was toward the epicenter. It is even possible that Earth reacts to every possible impactor, although the reaction may be proportional to impactor energy and thus usually negligible.

Also interesting about the Nicaragua event is that it occurred at the time of my birthday (September 1st, local time) producing an obvious signal[43] for me. I interpret this as a confirmation that the meteor was involved in this event.

Note that, at the point of writing of this paper, such sign[al]s are still not considered real by modern science (they are treated as mere coincidence). However, with $\mathrm{CR}$ the phenomena becomes not only real, but a [relatively] special form of synchronization and a driver of evolution with exponentially increasing significance near the end of an existence cycle. Thus, if one doesn't believe in signals of synchronicity (I didn't before I started experiencing them) I suggest one to study all my work, particularly the work referenced above.

Note that Nicaragua, Chelyabinsk and Tunguska impact sites on the world map can be connected with a straight line - a correlation suggesting that next impact may also occur somewhere along this line (even the Chicxulub, Yucatan crater is close).

Although there were no sightings of large meteors over land, a smaller magnitude impact was recorded on land area in 1992 - the Peekskill meteorite. It was recorded one month after the Nicaragua event and is notable for hitting a car in urban area.

Also interesting, and symbolic, is a fact that the last visit of the Halley's comet to the inner Solar System occurred at the time when $350 \mathrm{ppm}$ $\mathrm{CO}_{2}$ was first reached - in 1986., and the next time it will be close to Earth is 2061. - exactly at $650 \mathrm{ppm}$ (calculated using the C1.1 equation). It is currently hypothesized that Tunguska event was caused by a large body which eventually escaped Earth's atmosphere - it can thus be interpreted as a warning.

Given the fact that neither the Chelyabinsk nor hypothesized Nicaragua meteor did not directly impact land, it appears these too were warnings. However, I do not interpret these as warnings, I believe one purpose of the atmosphere is to disintegrate incoming bodies to protect life during weak evolution. Thus, much larger bodies and impacts should be expected only as events synchronized with strong evolution.

These recent events may thus be interpreted as signals of things to come. After these 3 misses, I believe next will be impacts.

Note that Newton calculated year 2060 as [the beginning of] the end of surface world, although he revised this year later to 2016 by the sugges- 
tion of others. His final decision to revise the year was, however, based on a signal. As he was doing calculations, large earthquake occurred which he later interpreted as a signal that the year 2060 is wrong. This earthquake was a signal, but he misinterpreted its meaning - large and frequent earthquakes are to be expected at the end.

The year 2016 is not there without a meaning for me though, it is the year of my soul rebirth (change of energy level) occurring at the age of 35 of the incarnation[44].

Note 3: Interestingly, at time of the Chelyabinsk event, Apophis asteroid was in close approach. Considering that the composition of Chelyabinsk meteor seems to match the composition of Apophis surface (LL chondrite) a probability exists that the meteor broke off of Apophis and is thus a part of impactor energy splitting.

Note 4: All above confirms that the initially chosen $50 \mathrm{ppm}$ quantum was good. I cannot attribute this to blind luck. The coherence of signals with my thoughts has been only growing ever since I've started experiencing them. Even prior to the models, based on intuition, I have felt years 2029 and 2066 as probable impact dates, and I wasn't even aware of Apophis at the time.

For me, the outcome of models and equations is often a confirmation of a signal rather than the other way around.

However, I will rarely mention signals [or use them as evidence] in my works, as I believe most of people do not sense or recognize them yet and even I could still misinterpret the signal. That is one of the reasons I use logic, equations and models to explain, analyze and predict phenomena. This, I consider necessary to fine-tune the coherence and signal interpretation.

Note 5: The equation $\mathrm{C} 1.1$ is one variant of a universal equation for a pulse of strong evolution. That $800 \mathrm{ppm}$ as the $\mathrm{CO}_{2}$ marker maximum was a good prediction can be confirmed with another variant (inverse) of the equation, one correlated with half-lives of elements:

$$
\begin{gathered}
T_{1 / 2}=2 C_{1}-\frac{C_{1}}{C O_{2}\left(T_{1}\right)} * C O_{2}=2 C_{1}-\frac{C_{1}}{C O_{2}\left(T_{1}\right)} * 300 *\left(\frac{6}{5} * 2^{45 x^{2}}\right)_{(\mathrm{C} 1.2)}^{x} \\
x=\frac{T-1905}{10 * 55}=\frac{T-1905}{550}
\end{gathered}
$$

where $C_{1}=T_{1 / 2}\left(T_{1}\right)$ is the half-life of the element measured at time $\mathrm{T}_{1}$. The equation gives half-life of 0 at, or near, $\mathrm{T}=2075$, which is the year when $\mathrm{CO}_{2}$ (T) is equal to $800 \mathrm{ppm}$. Just like in case of $\mathrm{CO}_{2}$ I do not expect for half-lives to follow the equation continuously (ie. half-life might appear constant and then get reduced significantly in an instant). Generally, changes in decay rates should require sudden changes in properties of space. 
One exception to this, in the Solar System, might be the half-life of ${ }^{10} \mathrm{Be}$, due to vertical entanglement with the $\mathrm{U}_{1}$ system. Solar System (scale $\mathrm{U}_{1}$ ) cycles through ${ }^{10}(\mathrm{C}-\mathrm{B}-\mathrm{Be})$ and I would expect a continuous precursor enrichment in $10 \mathrm{~B}$ at a lower scale $\left(\mathrm{U}_{0}\right)$ before the state change of $\mathrm{U}_{1}$.

For ${ }^{10} \mathrm{Be}$, incorporating the value from the most recent measurements $\left(\mathrm{T}_{1}=2010, \mathrm{~T}_{1 / 2}(2010)=1.387 * 10^{6} \mathrm{y}\right)$, the half-life equation is:

$$
T_{1 / 2}=2 * 1.387 * 10^{6}-\frac{1.387 * 10^{6}}{385.915461731} * 300 *\left(\frac{6}{5} * 2^{45 x^{2}}\right)^{x}
$$



Table 21: Calculation and measurements ${ }^{45}$ of ${ }^{10}$ Be half-life

* the value is not the initially published value, but the result of reanalysis/correction in

$\begin{aligned} & 1972 ., \\ & \dagger \text { these values are discarded by scientific community, citing potential systematic errors }\end{aligned}$ (based on the presumption of absolute constancy of decay rates).

All measurements agree well with calculated values, except for 1986 - if there were no flaws in measurement, this may be attributed to deviation due to cycling (similar to yearly fluctuation of $\mathrm{CO}_{2}$ ). Note, however, that measurement 1993 was done on the same SRM (Standard Reference Material) sample and discrepancy suggests one of these measurements is wrong.

If indeed the half-life of ${ }^{10} \mathrm{Be}$ is decreasing as hypothesized, modern science has been effectively doing cherry-picking here - discarding results which do not agree well, or are in discrepancy, with latest measurements.

Given the current precision of measurements, a new measurement at this point in time which would agree with the calculation would be in discrepancy with measurements from 2010. and would thus confirm the hypothesis of continuous decrease of ${ }^{10} \mathrm{Be}$ half-life prior to the extinction event.

Note that this effect on decay rates is temporary and significant only at the end of a cycle of general oscillation up to the 3rd order.

Note also that decay rates may not be changing directly (affecting half-life) rather effectively (CR requires effective oscillation in particle decay, but these changes will not always be reflected in half-life of the element) - ie. through spallation reactions.

Note 6: In the previous note it was assumed that half-life decreases fast and the equation allows it to eventually drop to zero (although, the compression of time implies that this state lasts 0 time - thus, effectively, half-life never becomes 0 ).

Another possibility, although unlikely, is that half-life cannot ever reach zero, even for 0 time. In that case, the equation might have this form:

$$
T_{1 / 2}=C_{1} * C O_{2}\left(T_{1}\right) * \frac{1}{C O_{2}}=C_{1} * C O_{2}\left(T_{1}\right) *\left[300 *\left(\frac{6}{5} * 2^{45 x^{2}}\right)^{x}\right]^{-1}
$$




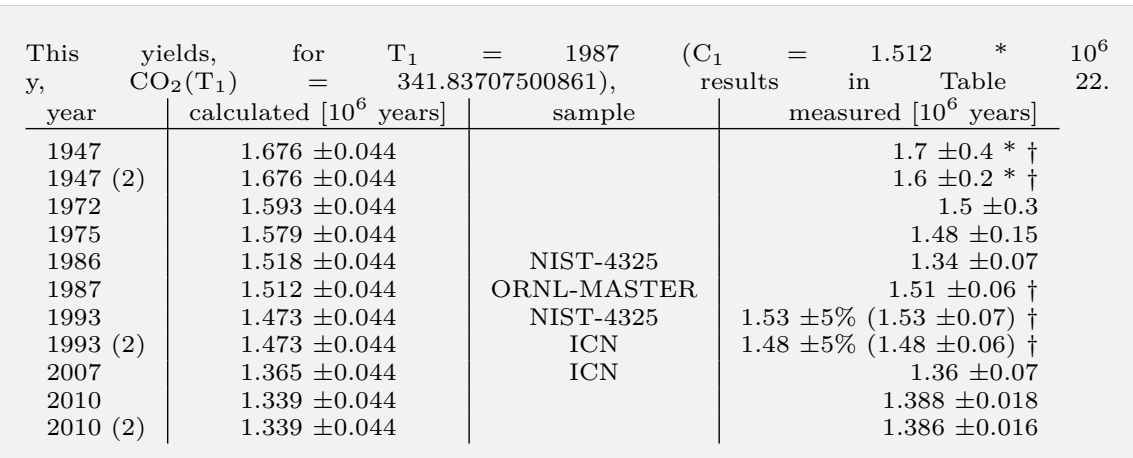

Table 22: Calculation and measurements of ${ }^{10} \mathrm{Be}$ half-life where error margin in calculation is the scaled variation of $\mathrm{CO}_{2}$ (10 ppm).

Such pulses might not only be plausible but necessary - first pulse would include asteroid impact(s) (possibly triggering additional ocean acidification and formation of the layer in the mantle), the other would be cometary (to provide new water/life). A third pulse in between might also be needed to trigger the (now acidified - CSF) ocean sink and, relatively, sterilize the surface.

Asteroid impacts may trigger, or coincide with, the gravitational maximum (incl. magnetic field) collapse and ocean (CSF) sink, allowing surface sterilization by $\mathrm{UV} /$ gamma radiation.

It might seem that a cometary impact this time is not needed - as formation of mantle layers should be complete with this extinction (corresponding to Carbon nature of the Solar System) there is no need for cultivation of new progenitor cells on surface. However, it probably does happen as it would provide additional radiation protection and provide support for whatever life remains on, or near, surface.

Note that a collapse of Moon's gravitational maximum is expected, remains of the Moon are thus the most likely source of eventual impacts of cometary nature (dust/water/ice).

This is evident on Mars - as layers below the surface formed, magnetic field receded leaving the surface sterilized. Delivered water froze and is now covered with dust. Thus, one can only expect to find residual and resilient bacteria within the crust of Mars.

Similar happened on Venus except water evaporated due to high surface temperature.

Nothing in nature is linear (although this approximation may be suitable during stages of weak evolution) and in these extreme events one can expect significant departures from linear relations (by multiple orders of magnitude) 
between phenomena.

Since these events are coupled with gravitational stresses of the Solar System one can expect temporary but significant increase in alpha and neutrino radiation (radiation flux induced by temporary collapse of a gravitational well - strongly affecting half-lives of isotopes).

One interpretation of changes in decay rates could be time dilation due to scale change of gravitational maximums.

Also note that these changes are synchronized with orbital decays in the Solar System - which, like the decay rates, are accelerated during the pulse but return to normal after the pulse.

Due to universal synchronization and restoration of previous equilibrium states it is hard to detect such pulses - in fact, astronomical and geological observations will not reveal any deviation from constancy of decay rates. Thus, with such nature of cataclysmic changes, the principle of uniformitarianism will inevitably seem, but cannot be, valid.

Note also that most of emitted radiation will be lost to space for the same reason - temporary collapse of gravitational/electro-magnetic well, thus solving the problem of missing radiogenic Helium[46]. Due to conservation of momentum, significant loss of heavier atmospheric particles is not expected due to well loss, but can occur during the short exposure to solar wind.

The assumption of constant decay rates will not only produce incorrect ages but can result in misplacement of events on a geological timescale. Thus, inconsistencies in certain geological records can serve as indirect evidence to disruptions in decay rates.



Figure 21: Neutrino pulse due to decay rate increase

Consider the neutrino pulse on Fig. 21 - under the assumption of constant decay rates, 3 different fossil records $\mathrm{A}, \mathrm{B}, \mathrm{C}$ may give following results:

- assuming non-isotropic space-time perturbation, such that fossil record A decay is not affected by the event at $t_{B}$, the event at $t_{B}$ (associated with 
fossil record B) might appear to have happened before the event at $t_{A}$ (associated with fossil record A)

- in case decay rates of both $\mathrm{A}$ and $\mathrm{B}$ are affected, the distance of $\mathrm{t}_{A}$ and $\mathrm{t}_{B}$ to $\mathrm{t}_{C}$ will be increased (time interval expansion)

Neutrino flux can also be decreased indicating shortening (rather than expansion) of time intervals, although in this context the increase of the flux is expected.

Due to accumulation, duration of fossilized events would apparently increase with time so older events would seem longer in duration compared to more recent events. This is exactly the case with current fossil evidence of past carbon cycle disruptions.

In such case, the current rate of $\mathrm{CO}_{2}$ injection is not different from those in previous major extinctions (the fact that it is anthropogenic makes no difference). If one assumes that the average period between extinctions is equal to the 2nd order oscillation period of the Solar System, in case of ideal synchronization, it is quantized by the 3rd order period of existence $\left(\mathrm{T}_{x}=1.512\right.$ * $10^{6}$ years). In such case, assuming the period must be roughly 26 or 27 million years, the proper period is:

$T_{d}=\left\lfloor\frac{26 * 10^{6}}{1.512 * 10^{6}}\right\rfloor 1.512 * 10^{6}=\left\lfloor\frac{27 * 10^{6}}{1.512 * 10^{6}}\right\rfloor 1.512 * 10^{6}=25.704 * 10^{6}$ years

This is in agreement with previously determined periodicity of impact cratering $\left(25.8 \pm 0.6 * 10^{6}\right.$ years $)[47]$.

One can now assume that the $\mathrm{CO}_{2}$ injection within the Cretaceous-Paleogene (K-Pg) boundary (66.5 - 65.5 mya) is equal to current injection (currently dominantly anthropogenic) and that increase of decay rate (effective compression of time, causing boundary to be significantly overestimated in duration) is induced within the boundary - with the start of boundary corresponding to $t_{A}$ and end to $\mathrm{t}_{C}$ on Fig. 21.

Assuming the $\mathrm{CO}_{2}$ increased from 780 ppmv to $1440 \mathrm{ppmv}\left(\Delta \mathrm{CO}_{2}=660\right.$ ppmv) in period 66.5 mya -65.5 mya $\left(\Delta \mathrm{t}_{i}=1\right.$ million years)[48], compression of time $\Delta \mathrm{t}_{c}$ with each major extinction is:

$$
\Delta t_{c}=\Delta t_{i}-\Delta t_{a i}=1 * 10^{6}-234=999766 \text { years }
$$

where $\Delta \mathrm{t}_{a i}$ is the period of 660 ppmv of anthropogenic $\mathrm{CO}_{2}$ increase since year 1850 (assuming this is the start of the new boundary), calculated using (C1.1).

Such compression of time is easily achievable using C1.2. In example, for ${ }^{10} \mathrm{Be}$ :

$$
T_{1 / 2}=2 * 1.512 * 10^{6}-\frac{1.512 * 10^{6}}{341.83707500861} * 300 *\left(\frac{6}{5} * 2^{45 x^{2}}\right)^{x}
$$

Half-life of ${ }^{10} \mathrm{Be}$ decreasing by the above equation, reaches required time 
compression in year 2065, on day 66 of the year. Source code:getage.php $+$

However, year 1850 as the start of the boundary is not convincing and recent research shows $\mathrm{CO}_{2}$ injection of $250 \mathrm{ppm}$, not $660 \mathrm{ppm}$, within the K-Pg boundary, though this does not affect compression $\left(\Delta \mathrm{t}_{c}\right)$ significantly (it makes it larger for a couple of decades at most).

Most likely start of a new boundary (end of Holocene) is year 2065 or 2066, which, with an increase of $250 \mathrm{ppm}$, gives year 2084 as the end, the same as in the previous assumption $(1850+234=2084)$.

Number of 3rd order cycles of existence since Cretaceous-Paleogene extinction event (66 mya):

$$
n=\left\lfloor\frac{t_{K P g}}{T_{x}}\right\rfloor=\left\lfloor\frac{66 * 10^{6}}{1.512 * 10^{6}}\right\rfloor=43
$$

Gravitational collapses during strong evolution pulses with a period of $\mathrm{T}_{x}$ years (3rd order period) may last only $\Delta \mathrm{t}_{n_{x}}=19.3$ seconds, but collapses during stronger evolution pulses occurring with a period of $\mathrm{T}_{d}$ years (2nd order) last longer (possibly 7 days).

With each extinction, gravitational collapse of the Sun releases the pressure from condensed energy beyond the surface event horizon and the Sun effectively starts expanding.

The expansion reaches the orbit of Mars before the gravitational well is restored, so, assuming expansion at the speed of light, time of increased decay radiation is:

$$
\Delta t_{n_{d}}=\frac{r_{M}}{c}=\frac{227.92 * 10^{9}}{2.99792458 * 10^{8}}=760.259 \mathrm{~s}=12.671 \mathrm{~m}
$$

where $\mathrm{r}_{M}$ is the distance of Mars to Sun.

Now one can calculate time compression with each cycle (pulse) of existence $\Delta \mathrm{t}_{c_{x}}$ and each extinction $\Delta \mathrm{t}_{c_{d}}$ :

$$
\begin{gathered}
\Delta t_{c}=\Delta t_{c_{d}}+\Delta t_{c_{x}} \\
\frac{\Delta t_{c_{d}}}{\Delta t_{c_{x}}}=\frac{\Delta t_{n_{d}}}{\Delta t_{n_{x}}} \\
\Delta t_{c_{x}}=\Delta t_{c} \frac{1}{\frac{\Delta t_{n_{d}}}{\Delta t_{n_{x}}}+1}=24751.794 \text { years }
\end{gathered}
$$




$$
\Delta t_{c_{d}}=\Delta t_{c}-\Delta t_{c_{x}}=975014.206 \text { years }
$$

Age of Earth is thus overestimated by:

$$
\sigma_{T_{E}}=\left\lfloor\frac{\Delta T_{E_{i m g}}}{T_{d}}\right\rfloor \Delta t_{c_{d}}+\left\lfloor\frac{\Delta T_{E_{i m g}}}{T_{x}}\right\rfloor \Delta t_{c_{x}}=245907386 \text { years }
$$

giving the real age of Earth:

$$
\Delta T_{E}=\Delta T_{E_{i m g}}-\sigma_{T_{E}}=4.29409 \pm 0.05 * 10^{9} \text { years }
$$

where $\Delta \mathrm{T}_{E_{i m g}}=4.54 \pm 0.05 * 10^{9}$ years.

If one assumes that $\mathrm{T}_{d}$ is the equivalent of 1 day of human embryo development, Earth is at the week 25 (GW25) of gestation period (right at the beginning, in case of corrected age).

The GW25 marks the end of embryonic neurogenesis in humans and thus agrees with the hypothesis of final major extinction.

The current carbon cycle disruption (6th major extinction) will thus not span thousands (10000) of years as predicted by the assumption of constant decay, but at most 234 years - starting from year 1850 (10000 years of already passed Holocene extinction may be regarded as a precursor to the major event starting at year 1850).

$$
1850+234=2084
$$

Note that this year corresponds to $950 \mathrm{ppm}$, as predicted by (C1.1).

Outer gravitational maximum of the Sun collapses on average every 1.512 million years. At that point the Sun is in a state of a white dwarf for 19.3 seconds before the well is restored.

This is a part of a normal ${ }^{10}(\mathrm{C}-\mathrm{B}-\mathrm{Be})$ oscillation cycle of a single atom in a low density environment.

A similar mechanism must be behind the longer lasting collapse happening on average every 25.704 million years. This, however, may be the exchange of gravitational maxima (gluons) between multiple atoms.

The other atom must cycle through states in a similar way - different atoms at each end of the chain, one with short lifetime, other with a long lifetime, and a shared decay product in between, which, at $\mathrm{U}^{0}$ has an extremely long (stable) half-life.

The long lifetime (half-life) at one end of this chain should be $25.704 *$ $10^{6}$ years.

However, most recent measurement of ${ }^{10} \mathrm{Be}$ half-life yielded $1.388 \pm 0.018$ myr[49] so one might assume that the measured half-life of this element too will be lower. The half-life to look for is thus:

$$
T_{1 / 2} \approx \frac{1.388 \pm 0.018}{1.512} * 25.704 * 10^{6}=23.596 \pm 0.018 * 10^{6} \mathrm{y}
$$


The half-life closest to this value is the one of ${ }^{236} \mathrm{U}$ (Uranium), which, according to measurement from 1980, has a half-life of $23.42 \pm 0.03 * 10^{6}$ years.

The decay product of ${ }^{236} \mathrm{U}$ is ${ }_{232} \mathrm{Th}$ (Thorium) so this isotope is in the middle of the chain.

Assuming horizontal conservation of half-life ratios, a total (short) halflife at the other end of the chain should be:

$$
T_{1 / 2}=\frac{19.3}{1.512} 25.704=328.1 \mathrm{~s}
$$

A plausible cycle is thus ( ${ }^{236} \mathrm{U}-$ ? $)-{ }^{232} \mathrm{Th}-\left({ }^{232} \mathrm{Ac}-{ }^{232} \mathrm{Ra}\right)$.

In any case the mass of this system is 23.6 Solar masses which means it oscillates between an $\mathrm{O}$ class star and an inactive state in the form of a neutron star or a black hole.

The source of such systems is the energetic disc concentrated around a galactic gluon (dark matter ring) in the galactic midplane through which Solar System passes roughly every 30 million years[50].

However, a direct interaction between such system and Solar is unlikely. A more plausible interaction is that of a Solar System and an alpha particle emitted during ${ }^{236} \mathrm{U}$ decay.

The alpha particle at $\mathrm{U}^{0}$ is stable so it's half-life at $\mathrm{U}^{1}$ is $\approx 0$, therefor it oscillates in a ${ }^{4}(\mathrm{H}-\mathrm{He}-\mathrm{Li})$ cycle.

Assuming gluon speed equal to c, distance from the Sun to alpha gluon at the point of gluon exchange must be equal to $1 \mathrm{MAU}$ (effective range of the strong force):

$$
c \Delta t_{n_{d}}=2.99792458 * 10^{8} * 760.259=227.92 * 10^{9} \mathrm{~m}
$$

The interaction causes orbital perturbations in the Solar System and may be a plausible source for predicted asteroid and cometary bombardment.

\subsubsection{Sea level changes}

Neurogenesis requires transfer of differentiated progenitor cells to subterranean world, into designated mantle layers. Therefor, a passageway must exist somewhere, connecting the surface with underground tunnels leading to such places - unless one is created when needed, which I find unlikely.

Scaling the largest neuron cells to Earth size, such passageway must have a radius of at least $\approx 250$ meters to allow sequential cell transfer. However, parallel transfer of multiple cells is certainly more plausible - a radius on the order of $10^{4} \mathrm{~m}$.

Thus, the only location where this could remain hidden (protected) and isolated when unused is Antarctica. Ice melting is required to expose this location 
but likely also to rise the sea level in order to pick up the proteins and cells on land area.

Rise in atmospheric greenhouse gases may not produce adequate rise in temperature required to melt ice in the predicted short time-frame. Thus, a different mechanism may be responsible to induce significant breaking and melting of ice sheets - volcanism/geothermal sources and possibly asteroid bombardment.

\subsubsection{Analysis of past extinctions}

Here, past extinctions are analyzed for periodicity, with incorporated corrections by previously calculated time compression due to pulses of decay rate changes.

Periodicity is obtained using circular spectral analysis[51] of a couple of datasets, which all give similar results.

Data is grouped into energy levels corresponding to the extinction magnitude (5 - major extinctions, 4 - minor extinctions, 3 - other extinctions, 2 and 1 potential extinctions).

The method In the circular model of periodicity a time line is wrapped around a circle, the circumference of which represents a trial period. For each occurrence, a unit vector from the origin is calculated. If periodic, the series will tend to form a cluster at one point on the circumference when the correct trial period is used. Here, angular location relative to $0^{\circ}$ (present) gives the phase $\left(t_{0}\right)$.

Ages of individual events $\left(\mathrm{t}_{i}\right)$ are transformed to angles $\left(\mathrm{a}_{i}, \mathrm{~b}_{i}\right)$ for each trial period $\mathrm{P}$ :

$$
\begin{aligned}
a_{i} & =\sin \left(\frac{2 \pi}{P} t_{i}\right) \\
b_{i} & =\cos \left(\frac{2 \pi}{P} t_{i}\right) \\
S & =\frac{1}{N} \sum_{i=1}^{N} a_{i} \\
C & =\frac{1}{N} \sum_{i=1}^{N} b_{i} \\
R & =\sqrt{S^{2}+C^{2}}
\end{aligned}
$$

where $\mathrm{R}$ is a mean vector magnitude (normalized measure of goodness of fit). The phase shift $\left(t_{0}\right)$ is calculated as follows:

$$
\begin{gathered}
t_{0}=\frac{P}{2 \pi} \tan ^{-1}\left(\frac{S}{C}\right) \quad(\text { for } C>0) \\
t_{0}=\frac{P}{2}+\frac{P}{2 \pi} \tan ^{-1}\left(\frac{S}{C}\right) \quad(\text { for } C<0)
\end{gathered}
$$




\begin{tabular}{l|l|l}
$\begin{array}{l}\text { energy } \\
\text { level }\end{array}$ & extinction events [mya] & extinction events $\left(\mathrm{t}_{i}\right)$, age corrected [mya] \\
\hline 5 & $66^{*}, 201.3^{*}, 252.2^{*}, 365,445$ & $61.986,190.208,238.316,345.385,421.148$ \\
4 & $37.8^{*}, 145^{*}, 260^{a}, 305^{b}, 420^{c}$ & $36.206,136.774,245.993,288.3,397.519$ \\
3 & $11.6^{*}, 93.9^{*}, 182.7^{*}, 230^{d}$, & $11.402,88.465,172.88,217.463,255.844,401.469$, \\
& $270,424^{e}, 428^{f}, 488^{g}, 502$ & $404.42,461.48,475.257$ \\
2 & $117^{h}, 168.3^{*}$ & $111.194,159.702$
\end{tabular}

Table 23: Extinction events dataset 1, sources: ${ }^{* 52}, \mathrm{a}^{53}, \mathrm{~b}^{54}, \mathrm{c}^{55}, \mathrm{~d}^{56}, \mathrm{e}^{57}, \mathrm{f}^{58}$, $\mathrm{g}^{59}, \mathrm{~h}^{60}$

Dataset 1 Extinction events in dataset 1, grouped into energy levels and calculated corrected ages for these events, respectively, are shown in Table 23.

Maximal $\mathrm{R}$ was obtained for a period $\mathrm{P}=25.92 \mathrm{My}$ (million years), with a phase of $9.355 \mathrm{My}$.
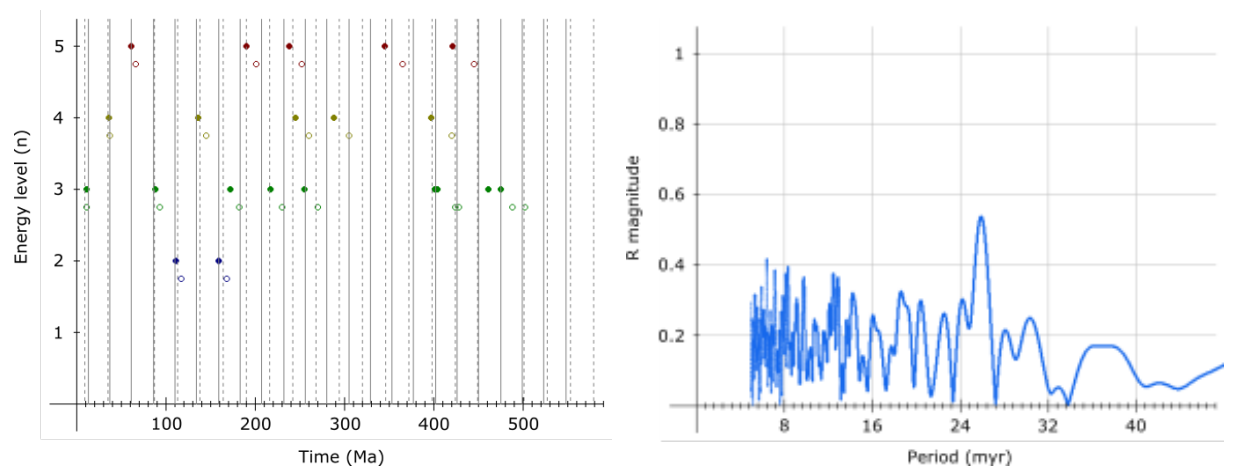

Figure 22: Extinctions (left), spectral analysis (right)

On the left, Fig. 22 shows extinctions plotted against the obtained periodicity (dashed gray line), solid colored circles are extinction events with corrected ages, empty circles are extinctions with non-corrected ages. On the right, Fig. 22 shows the result of circular spectral analysis.

Dataset 2 Here, a larger dataset from a single source was used. Maximal R

\begin{tabular}{l|l|l}
$\begin{array}{l}\text { energy } \\
\text { level }\end{array}$ & extinction events [mya] & extinction events $\left(\mathrm{t}_{i}\right)$, age corrected [mya] \\
\hline 5 & $66,201.4,251.9,372.2,445.2$ & $61.986,190.308,238.041,352.461,421.348$ \\
4 & $37.8,145,259.8,306.7,419.2$ & $36.206,136.774,245.793,289.975,396.744$ \\
3 & $11.6,93.9,183.7,228.5,272.3$, & $11.402,88.465,173.88,215.987,257.12,400.469$, \\
& $423,427.4,485.4,500.5$ & $403.82,458.929,473.782$ \\
2 & $113.1,168.3$ & $107.344,159.702$
\end{tabular}

Table 24: Extinction events dataset 2, source: Gradstein2016 ${ }^{61}$

reveals a period $\mathrm{P}=26 \mathrm{My}$, with a phase of $8.617 \mathrm{My}$.

Extinctions and the result of spectral analysis are shown in Fig. 23. 

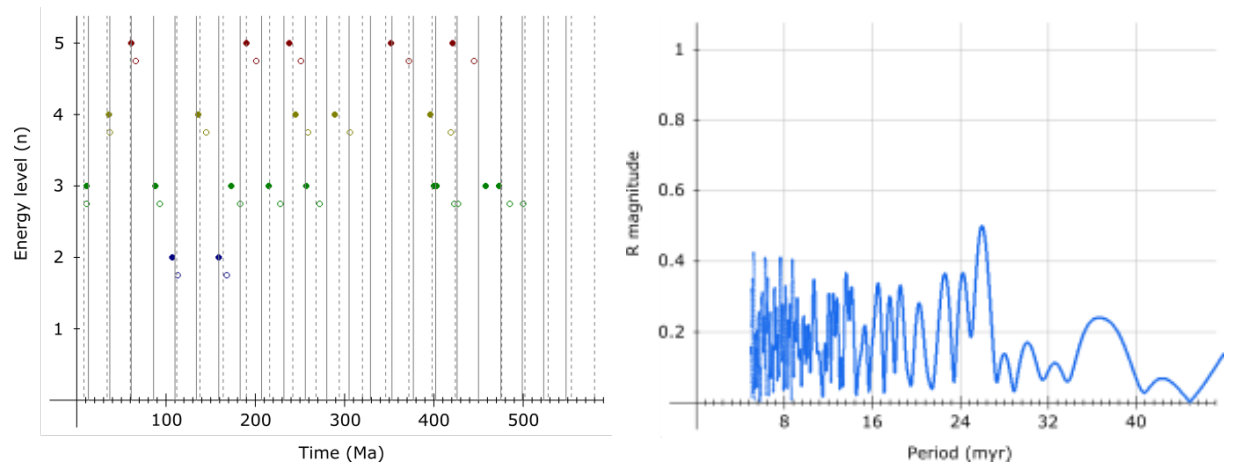

Figure 23: Extinctions (left), spectral analysis (right)

Dataset 3 Previous datasets do not take into account possible splitting of energy levels. Here, an even larger dataset is presented which shows possible energy splitting and how this, when not accounted for, causes lower confidence in calculated $\mathrm{P}$.

\begin{tabular}{l|l|l}
$\begin{array}{l}\text { energy } \\
\text { level }\end{array}$ & extinction events [mya] & extinction events $\left(\mathrm{t}_{i}\right)$, age corrected [mya] \\
\hline 5 & $66,201.4,251.9,372.2,445.2$ & $61.986,190.308,238.041,352.461,421.348$ \\
4 & $37.8,145,259.8,306.7,419.2$, & $36.206,136.774,245.793,289.975,396.744,486.084$ \\
& 514 & \\
3 & $11.6,93.9,183.7,228.5,272.3$, & $11.402,88.465,173.88,215.987,257.12,400.469$, \\
& $423,427.4,485.4,500.5,541$ & $403.82,458.929,473.782,511.664$ \\
2 & $113.1,168.3,330.9$ & $107.344,159.702,312.804$ \\
1 & $295,346.7,393.3,467.3$ & $279.448,328.357,372.239,442.101$
\end{tabular}

Table 25: Extinction events dataset 3, source: Gradstein2016 ${ }^{62}$

Here, for $\mathrm{R}=0.413$, obtained $\mathrm{P}=22.493 \mathrm{My}$, phase $15.603 \mathrm{My}$.

Dataset 4 Here I hypothesize that deviations from $\mathrm{P}$ are the result of energy splitting into smaller events which when grouped properly would fit on $\mathrm{P}$ intervals.

The dataset is the same as dataset 3, except the hypothesized splittings (circled extinction pairs on Fig. 24) have been grouped into a single event, simply by using arithmetic mean age of the pair.

The $\mathrm{R}$ peaks at 0.807 , corresponding to $\mathrm{P}=25.89 \mathrm{My}$, very close to one obtained from dataset 1. Phase is $9.55 \mathrm{My}$.

Dataset 5 Here dataset 4 is modified with the assumption that splitting occurs in all events, thus, in addition to previously grouped events, the remaining non-grouped events have been grouped with adjacent boundaries.

The $\mathrm{R}$ peaks at 0.75 , corresponding to $\mathrm{P}=25.84 \mathrm{My}$. Phase for this $\mathrm{P}$ is $9.78 \mathrm{My}$, however, here another peak at $12.875 \mathrm{My}(\mathrm{R}=0.61)$ reveals a likely harmonic. 




Figure 24: Extinctions


Figure 25: Extinctions (left), spectral analysis (right) 


\begin{tabular}{l|l|l}
$\begin{array}{l}\text { energy } \\
\text { level }\end{array}$ & extinction events [mya] & extinction events $\left(\mathrm{t}_{i}\right)$, age corrected [mya] \\
\hline 5 & $66,201.4,(251.9+259.8) / 2=$ & $61.986,190.308,241.967,352.461,421.348$ \\
4 & $255.9,372.2,445.2$ & \\
& $37.8, \quad 145, \quad 306.7$, & $36.206,136.774,289.975,398.619,499.361$ \\
& $(419.2+423) / 2=421.1$, & \\
3 & $(514+541) / 2=527.5$ & \\
& $11.6,93.9,(183.7+168.3) / 2=$ & $11.402,88.465,166.304,215.987,268.346,403.82$ \\
& $176,228.5,(272.3+295) / 2=$ & $451.053,473.782$ \\
2 & $283.7,427.4,(485.4+467.3) / 2$ & \\
1 & $=476.4,500.5$ & \\
& $113.1,(330.9+346.7) / 2=339$ & $107.344,320.78$ \\
& 393.3 &
\end{tabular}

Table 26: Extinction events dataset 4

\begin{tabular}{|c|c|c|c|}
\hline $\begin{array}{l}\text { energy } \\
\text { level }\end{array}$ & \multicolumn{2}{|l|}{ extinction events [mya] } & extinction events $\left(t_{i}\right)$, age corrected [mya] \\
\hline 5 & $\begin{array}{lll}(61.6+66) / 2 & = \\
(199.4+201.4) / 2 & = & 2 \\
(251.9+259.8) / 2 & = & 2 \\
(372.2+382.7) / 2 & = & 3 \\
(443.8+445.2) / 2 & = & 444.5\end{array}$ & $\begin{array}{l}63.8, \\
200.4 \\
255.9 \\
377.5 \\
5\end{array}$ & $60.81,189.333,241.967,356.687,420.648$ \\
\hline 4 & $\begin{array}{lll}(33.9+38) / 2= & \\
(139.4+145) / 2= & 1 \\
(306.7+314.6) / 2= & 3 \\
(419.2+423) / 2= & 4 \\
(514+541) / 2=527.5 & \end{array}$ & $\begin{array}{r}36, \\
142.2 \\
310.7 \\
421.1\end{array}$ & $34.431,134.998,293.926,398.619,499.361$ \\
\hline 3 & $\begin{array}{l}(11.6+13.8) / 2= \\
(89.8+93.9) / 2= \\
(183.7+168.3) / 2= \\
(228.5+237) / 2=2 \\
(272.3+295) / 2=2 \\
(427.4+430.5) / 2= \\
(485.4+467.3) / 2= \\
(497+500.5) / 2=498.8\end{array}$ & $\begin{array}{r}12.7 \\
91.9 \\
176 \\
232.8 \\
283.7 \\
429 \\
476.4\end{array}$ & $\begin{array}{l}12.502,86.49,166.304,220.213,268.346,405.395 \\
451.053,472.107\end{array}$ \\
\hline 2 & $\begin{array}{l}(113.1+126.3) / 2=1 \\
(330.9+346.7) / 2=339\end{array}$ & 119.7, & $112.87,320.58$ \\
\hline 1 & $(387.7+393.3) / 2=390.5$ & & 369.489 \\
\hline
\end{tabular}

Table 27: Extinction events dataset 5

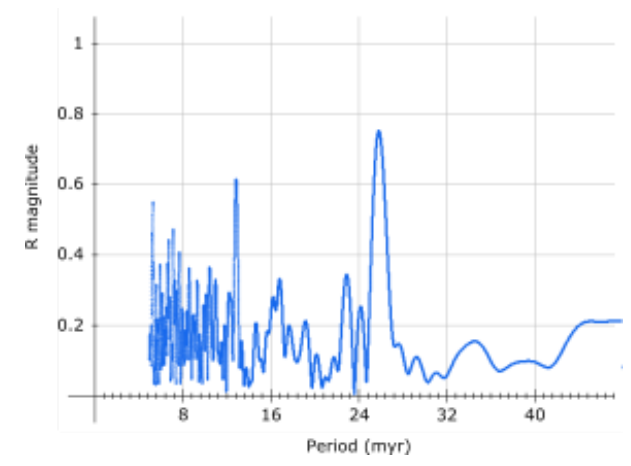

Figure 26: Dataset 5, spectral analysis 
Dataset 6 Here, dataset contains only highest energy (major and minor) extinctions, from dataset 1 . This dataset gives highest $\mathrm{R}$ maximum (0.837), a

\begin{tabular}{l|l|l}
$\begin{array}{l}\text { energy } \\
\text { level }\end{array}$ & extinction events [mya] & extinction events $\left(\mathrm{t}_{i}\right)$, age corrected [mya] \\
\hline 5 & $66,201.3,252.2,365,445$ & $61.986,190.208,238.316,345.385,421.148$ \\
4 & $37.8,145,260,305,420$ & $36.206,136.774,245.993,288.3,397.519$
\end{tabular}

Table 28: Extinction events dataset 6

period $\mathrm{P}=25.74 \mathrm{My}$, with a phase of $9.689 \mathrm{My}$.

Confidence Note that equal weight was assumed for all extinctions in a particular dataset. Different weights can affect the confidence in the result (less if they are all harmonics). But even with that taken into account, there is high confidence in $\mathrm{P} \approx 25.74 \mathrm{My}-25.89 \mathrm{My}$.

The result with highest confidence $(25.74 \mathrm{My})$ is also the closest to calculated ideal quantization by the 3rd order period $\left(1.512 * 10^{6} \mathrm{My}\right)-25.704 \mathrm{My}$, further increasing confidence in such periodicity.

Note that the burning cycle of the Sun's core is calculated (in the "Quantization of the Sun" chapter") to be equal to 25.746608 My, confirming the signal.

Neurogenesis in lifeforms on Earth during embryonic development does imply certain periodicity in the formation of brain layers and neuron migration.

High energy impact cratering and extinctions (migrations) in planetary neurogenesis should be no exception.

In fact, with such periodicity and the last high energy extinction $37.8 \mathrm{My}$ in the past, next one is overdue, roughly by the phase shift.

Note that such delay of extinction may have some benefits due to more evolved precursor neurons at time of differentiation, although with the cost of increased probability of cancer development.

Thus, imminent major extinction as calculated using models based on C1.1 equation should not be surprising.

Correlation with mantle layers Grouping and correlation of extinction events with the formation of brain [mantle] layers also indicates that another major mass extinction should be near, at least in geological terms.

This correlation is shown on Fig. 27 - time between major extinction events of Phanerozoic is proportional to thickness of a corresponding mantle layer.

Such correlation should not be surprising - all lifeforms grow in layers. 

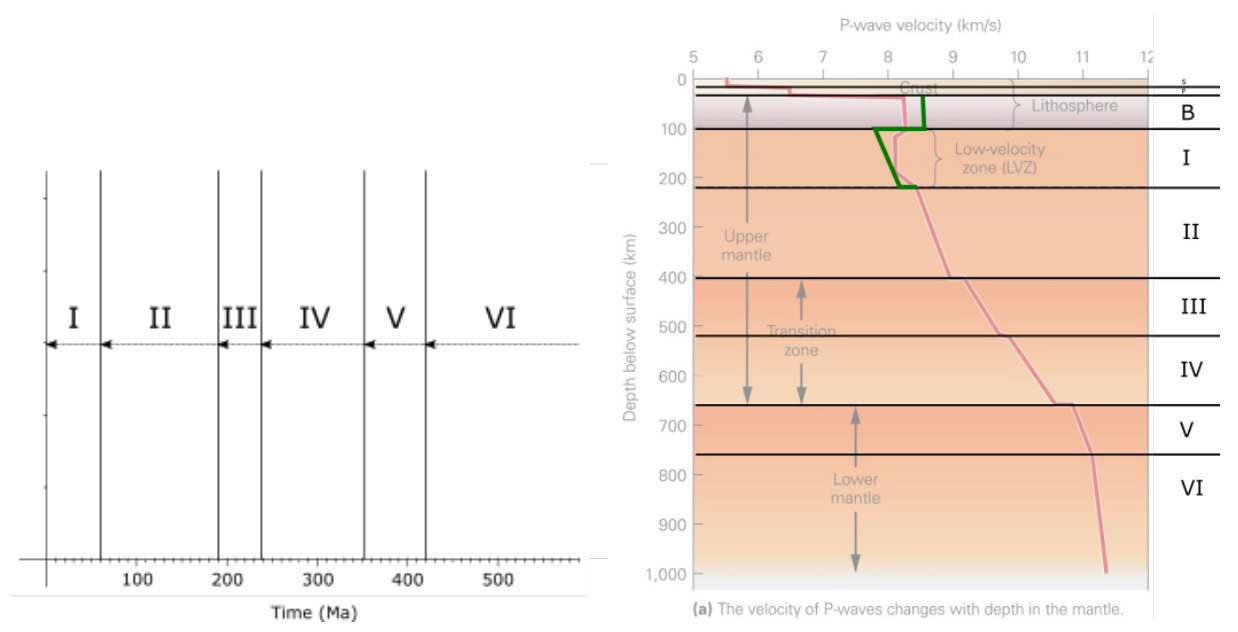

Figure 27: Correlation of major extinctions (left) with Earth's mantle layers (right)

This is, effectively, a conversion of time separated discontinuities into events separated in space.

To quantify the correlation, periods of weak evolution of mantle layers and thicknesses have been normalized:

$$
\begin{gathered}
T_{n}(i)=\frac{T(i)}{\sum_{j=1}^{N} T_{j}} \\
D_{n}(i)=\frac{D(i)}{\sum_{j=1}^{N} D_{j}}
\end{gathered}
$$

Results are shown in Table 29. Here, corrected extinction ages are used, although non-corrected ages would yield similar results. Correlation in absolute value varies between the pairs, but qualitatively, correlation is very good.

At least some discrepancies could be explained by the fact that formation is not yet complete - ie. the boundary between layers 3 and 4 may change with the pending extinction.

If layer 3 decrease would be equal to layer 4 increase $(\approx 0.0575$ in normalized value) and layer 1 decrease to layer 2 increase $(\approx 0.0275 \approx 0.0575 / 2)$, with a small decrease in layer $5(0.013 \approx 0.0275 / 2)$ coupled with equivalent increase in layer 6 , normalized extinction and mantle boundaries would be almost equal.

This suggests that extinction events are memorized, all layers change with every extinction but toward a specific predetermined pattern - ex- 


\begin{tabular}{l|l|l|l|l}
$\mathrm{i}$ & $\begin{array}{l}\text { Period of weak evolution } \\
\mathrm{T}[\mathrm{My}]\end{array}$ & $\begin{array}{l}\text { Normalized } \\
\text { period of } \\
\text { weak evo- } \\
\text { lution } \mathrm{T}_{n}\end{array}$ & $\begin{array}{l}\text { Corresponding } \\
\text { mantle layer } \\
\text { thickness D [km] }\end{array}$ & $\begin{array}{l}\text { Normalized } \\
\text { layer } \\
\text { thick- } \\
\text { ness } \mathrm{D}_{n}\end{array}$ \\
\hline 5 & $421.348-352.461=68.887$ & 0.163 & $780^{b}-660^{b}=120$ & 0.176 \\
4 & $352.461-238.041=114.42$ & 0.272 & $660^{b}-520^{b}=140$ & 0.206 \\
3 & $238.041-190.308=47.733$ & 0.113 & $520^{b}-410^{b}=110$ & 0.162 \\
2 & $190.308-61.986=128.322$ & 0.305 & $410^{b}-220^{a}=190$ & 0.279 \\
1 & $61.986-0=61.986$ & 0.147 & $220^{a}-100^{b}=120$ & 0.176
\end{tabular}

Table 29: Comparison of weak evolution periods and mantle layers, sources: $a^{63}, b^{64}$

actly as expected with DNA encoded evolution of living organisms.

Note that exact location of boundaries is a matter of debate. They must have some thickness, so it may be more appropriate to equate layer thickness with distance between discontinuities. If that would be a distance between lower discontinuities of two boundaries, it would, for layer 1, yield a normalized value exactly equal to the corresponding normalized period of weak evolution:

$$
\frac{220-120}{680}=\frac{100}{680}=0.147
$$

Also, globally average velocities might not be the best choice for determination of layer discontinuities - ie. Lehmann discontinuity is at $220 \mathrm{~km}$ for tectonic North America, but $200 \mathrm{~km}$ for shield North America[65], while it may be absent beneath north Atlantic and other oceans.

No graviton can be completely neutral. If discontinuities are [large scale] gravitons, holes in these, proportional to polarization, are expected.

If one assumes that $200 \mathrm{~km}$ is a real boundary, while $220 \mathrm{~km}$ is a precursor boundary and will reduce to $200 \mathrm{~km}$ with complete formation, the correlation with extinctions for both layers, I and II, becomes remarkable:

$$
\begin{aligned}
& \frac{200-100}{680}=\frac{100}{680}=0.147 \\
& \frac{410-200}{680}=\frac{210}{680}=0.309
\end{aligned}
$$

Some report the base of the upper mantle at $670 \mathrm{~km}[66]$ rather than 660 , this 
improves the correlation with layers 5 and 4 :

$$
\begin{aligned}
& \frac{780-670}{680}=\frac{110}{680}=0.162 \\
& \frac{670-520}{680}=\frac{150}{680}=0.221
\end{aligned}
$$

Now, the only problematic boundary is the one between layers 3 and 4 (at $520 \mathrm{~km}$ ). Some do report this boundary at $500 \mathrm{~km}$, which gives much better agreement:

$$
\begin{aligned}
& \frac{670-500}{680}=\frac{170}{680}=0.25 \\
& \frac{500-410}{680}=\frac{90}{680}=0.132
\end{aligned}
$$

Note that extinction boundaries also have some thickness or uncertainties, notably first three, which may explain differences in reported discontinuity depths. The 3rd major extinction (Permian) is apparently split into two events (EndCapitanian and Permian-Triassic). Using End-Capitanian 245.793 Mya (259.8 Mya non-corrected) instead of Permian-Triassic 238.041 Mya (251.9 Mya noncorrected) as the date of Permian extinction gives results in remarkable agreement with the obtained layers 3 and 4 (with discontinuities at $410 \mathrm{~km}, 500 \mathrm{~km}$ and $670 \mathrm{~km})$ :

$$
\begin{aligned}
& \frac{352.461-245.793}{421.348}=\frac{106.668}{421.348}=0.253 \\
& \frac{245.793-190.308}{421.348}=\frac{55.485}{421.348}=0.132
\end{aligned}
$$

Correlation of layer 6 and the corresponding period of weak evolution has not been determined due to unknown boundary.

However, assuming the extinction at the start of Phanerozoic (511.664 mya in corrected age, or 541 mya non-corrected) is correlated with the lower boundary of layer 6 , one can calculate the thickness of layer 6 :

$$
\begin{gathered}
\frac{T_{6}}{T_{5}}=\frac{D_{6}}{D_{5}} \\
D_{6}=\frac{T_{6}}{T_{5}} D_{5}=\frac{511.664-421.348}{421.348-352.461} 120=157 \mathrm{~km}
\end{gathered}
$$

In that case, a discontinuity, if formed, should exist in Earth's mantle at a depth of $937 \mathrm{~km}$ (assuming boundary between layer 5 and 6 at $780 \mathrm{~km}$ ).

Apparently, this discontinuity has been detected[67] (at $940 \mathrm{~km})$.

Supplement Here is the code used to calculate correct ages of extinction events, perform the analysis and generate images.getext.php + 


\section{Quantization of Moon orbits}

If the gravity of Earth's [major] gravitational maximum is, as hypothesized, equal to surface gravity of the Sun, one would expect for orbitals of natural moons of Earth to be scaled orbitals of inner planets.

Allowed orbitals are thus:

$$
r=\frac{r_{p}}{R_{\odot}} r_{c}
$$

where $r_{c}$ is the Earth's gravitational maximum radius (= inner core radius), $R_{\odot}$ is the radius of the Sun and $r_{p}$ is the orbital radius of a corresponding planet.

Using $\mathrm{R}_{\odot}=695735 \mathrm{~km}, \mathrm{r}_{c}=1206.115 \mathrm{~km}$, one obtains orbitals shown in Table 30. Evidently, the Moon is currently at the scaled Mars' orbit. Even the

\begin{tabular}{l|c|c} 
entanglement & $\mathrm{r}_{p}(\mathrm{~km})$ & $\mathrm{r}(\mathrm{km})$ \\
\hline Mercury & 57910000 & 100392 \\
Venus & 108210000 & 187591 \\
Earth & 149600000 & 259344 \\
Mars & 227920000 & 395118
\end{tabular}

Table 30: Allowed orbitals of the Moon

distance between perihelion and aphelion is scaled by equal orders of magnitude - for Mars it is $42.61 * 10^{6} \mathrm{~km}$, while for the Moon, the distance is $42.2 * 10^{3}$ $\mathrm{km}$.

Small discrepancies should be attributed to oscillation and phase shift in synchronization.

Note that Earth is likely receding from the Sun at the scaled rate of Moon's recession from Earth.

\section{Quantization of the Sun}

During inflation of the Sun, multiple gravitational maxima were inflating within. Collapse of these maxima as the Sun was deflating was fossilized in the Sun, in the form of discontinuities. As these maxima are now gravitational maxima of inner planets, entanglement exists between radii of discontinuities and planetary orbits.

Some discontinuities are strong (permanent) while some are weak, evolve over time and may periodically disappear. Apparent discontinuities are those between the core, radiative and convective zone, surface discontinuity and the boundaries of tachocline. 
Regardless of the configuration $\left(1 \mathrm{e}^{+}\right.$or $\left.2 \mathrm{e}^{+}\right)$, each inner planet formed with the collapse of two neutral spin anti-aligned maxima. Thus, each is entangled with 2 discontinuities in the Sun.

Initial inflation of planetary maxima must have been faster than light to preserve invariance.

If one assumes that all maxima initially had the mass of the Sun and energy density remained constant during inflation, with the collapse (energy level change) occurring once escape velocity was equal to the speed of light (in CR, discontinuities between energy levels are speed limits), orbital radii of planets become fossils of Schwarzschild radii:

$$
\begin{gathered}
r=\frac{2 G m}{c^{2}}=\frac{2 G \rho V}{c^{2}}=\frac{r^{3}}{R^{3}} \frac{2 G M}{c^{2}} \\
r=\sqrt{R^{3} \frac{c^{2}}{2 G M}} \\
\mathrm{R}=\text { initial radius } \\
\mathrm{M}=1.988500^{*} 10^{30} \mathrm{~kg} \\
\mathrm{c}=\text { standard speed of light }=2.99792458^{*} 10^{8} \mathrm{~m} / \mathrm{s} \\
\mathrm{G}=6.674^{*} 10^{-11} \mathrm{~m}^{3} / \mathrm{kgs}^{2}
\end{gathered}
$$

With equal escape velocity (pressure per surface quantum) between maxima (note that a smaller maximum is inside the other), radius of fusion of two maxima becomes the arithmetic mean of two radii:

$$
r=\frac{1}{2}\left(\sqrt{R_{1}^{3} \frac{c^{2}}{2 G M}}+\sqrt{R_{2}{ }^{3} \frac{c^{2}}{2 G M}}\right)
$$

In that case, discontinuities entangled with planetary orbits are at $1 / 5 \mathrm{R}_{\odot}, 2 / 5$ $\mathrm{R}_{\odot}, 1 / 2 \mathrm{R}_{\odot}, 2 / 3 \mathrm{R}_{\odot}$ and $1 \mathrm{R}_{\odot}$. Correlation of orbital and Schwarzschild radii

\begin{tabular}{l|c|c|l|l|l|l} 
Planet & $\mathrm{R}_{1}$ & $\mathrm{R}_{2}$ & $\begin{array}{l}\text { Schwarzschild ra- } \\
\text { dius } \mathrm{r}\left(10^{6} \mathrm{~km}\right)\end{array}$ & $\begin{array}{l}\text { current orbital ra- } \\
\text { dius }\left(10^{6} \mathrm{~km}\right)\end{array}$ & $\begin{array}{l}\text { orbital radius } \\
(\mathrm{MAU})\end{array}$ \\
\hline Mars & $\mathrm{R}_{\odot}$ & $1 / 2 \mathrm{R}_{\odot}$ & 228.52 & 227.92 & 1 \\
Earth & $2 / 3 \mathrm{R}_{\odot}$ & $1 / 2 \mathrm{R}_{\odot}$ & 151.59 & 149.6 & $2 / 3$ \\
Venus & $2 / 3 \mathrm{R}_{\odot}$ & $1 / 5 \mathrm{R}_{\odot}$ & 107.00 & 108.21 & $1 / 2$ \\
Mercury & $2 / 5 \mathrm{R}_{\odot}$ & $1 / 5 \mathrm{R}_{\odot}$ & 57.81 & 57.91 & $1 / 4$
\end{tabular}

Table 31: Correlation of orbital and Schwarzschild radii

is shown in Table 31 , where $R_{\odot}$ is the radius of the Sun $(695700 \mathrm{~km})$.

Significant orbital eccentricity of Mercury and Mars also seems correlated with Sun's discontinuities.

If Sun's core radius oscillates between $0.1+0.186 \mathrm{R}_{\odot}=0.286 \mathrm{R}_{\odot}$ (hypothesized initial radius) and $1 / 5 \mathrm{R}_{\odot}$ (current radius), with constant energy density between the two radii, time independent core radius [as superposition of two oscillatory states] is at $1 / 4 \mathrm{R}_{\odot}$. 
This is correlated with Mercury's orbit, as its distance from the Sun is at $1 / 4$ MAU, while its perihelion is at $1 / 5$ MAU.

According to equation S1.1 describing rotational velocities of plasma, and the actual velocity curve, significant points are at $0.1 \mathrm{R}_{\odot}, \approx 1 / 2 \mathrm{R}_{\odot}, 1+0.18686$ $\mathrm{R}_{\odot}=1.18686 \mathrm{R}_{\odot}$ and $32.8 \mathrm{R}_{\odot}(0.1 \mathrm{MAU}$, half of Mercury's perihelion).

The aphelion of Mars is at $1+0.18686 / 2 \mathrm{MAU}=1.09343 \mathrm{MAU}=249.2 *$ $10^{9} \mathrm{~m}$.

Note that the aphelion of Mars can also be obtained as volumetric mean of Schwarzschild radii associated with 3 discontinuities:

$$
\begin{gathered}
r^{3}=\frac{1}{3}\left\{\left[\left(1 R_{\odot}\right)^{3} \frac{c^{2}}{2 G M}\right]^{\frac{3}{2}}+\left[\left(\frac{2}{3} R_{\odot}\right)^{3} \frac{c^{2}}{2 G M}\right]^{\frac{3}{2}}+\left[\left(\frac{1}{2} R_{\odot}\right)^{3} \frac{c^{2}}{2 G M}\right]^{\frac{3}{2}}\right\} \\
r=249.2 * 10^{9} \mathrm{~m}
\end{gathered}
$$

Similarly, approximate aphelions can be obtained for other planets, ie. for Mercury:

$$
\begin{gathered}
r^{3}=\frac{1}{2}\left\{\left[\left(\frac{2}{5} R_{\odot}\right)^{3} \frac{c^{2}}{2 G M}\right]^{\frac{3}{2}}+\left[\left(\frac{1}{4} R_{\odot}\right)^{3} \frac{c^{2}}{2 G M}\right]^{\frac{3}{2}}\right\} \\
r=70.4 * 10^{9} \mathrm{~m}
\end{gathered}
$$

\subsection{Layers of the Sun}

Internal gravity of the Sun depends on the location of maxima and acquired real mass.

Distribution of mass, however, should not be complex unless there are collapsed large scale maxima inside. In any case, matter accumulated between two maxima should, in equilibrium, imitate a maximum and can thus be approximated as one (induced maximum).

One way to obtain gravity of a primordial Sun is to derive it from rotation of real mass - assuming greater rotation with greater gravitational mass, down to the inner core radius $\mathrm{r}_{c}$, quantization is 1-dimensional (negative):

$$
\frac{1}{g} v r=n h_{2}
$$

Giving the scaled h constant:

$$
h=h_{2}=5 * 10^{9} \mathrm{~ms}
$$




$$
n=1
$$

Another way is to assume a completely naked Sun, in which case gravity from the surface down to the core is:

$$
g=g_{p}=G M_{\odot} \frac{r^{2}}{R_{\odot}{ }^{4}}=274 \frac{r^{2}}{R_{\odot}{ }^{2}}
$$

Gravitational profile of the primordial Sun (not taking into account the gravity of inner core maximum) is given in Table 32. Here matter velocity (v) is extrapolated from measurements, while space velocity $\left(\mathrm{v}_{s}\right)$ is calculated from gravity:

$$
\begin{gathered}
v_{s}=\sqrt{g r} \\
v_{p}=\sqrt{g_{p} r}
\end{gathered}
$$

\begin{tabular}{|c|c|c|c|c|c|c|c|c|c|}
\hline $\mathrm{n}$ & $\mathrm{r} / \mathrm{R}$ & note & $\begin{array}{l}\text { space ve- } \\
\text { locity v }{ }^{2} \\
(\mathrm{~km} / \mathrm{s})\end{array}$ & $\begin{array}{l}\text { space ve- } \\
\text { locity v } v_{S} \\
(\mathrm{~km} / \mathrm{s})\end{array}$ & $\begin{array}{l}\text { matter } \\
\text { velocity } v \\
(\mathrm{~m} / \mathrm{s})\end{array}$ & $\begin{array}{l}\text { orbital } \\
\text { ra- } \\
\text { dius } \mathrm{r} \\
(\mathrm{km})\end{array}$ & $\begin{array}{l}\text { calculated } \\
\text { grav- } \\
\text { ity } g_{p} \\
\left(\mathrm{~m} / \mathrm{s}^{2}\right)\end{array}$ & $\begin{array}{l}\text { calculated } \\
\text { grav- } \\
\text { ity } \mathrm{g} \\
\left(\mathrm{m} / \mathrm{s}^{2}\right)^{2}\end{array}$ & $\begin{array}{l}\text { gravity } \\
\mathrm{g}_{i} \quad\left(\mathrm{v}_{c} \mathrm{r}\right. \\
\text { product) } \\
\mathrm{m} / \mathrm{s}^{2}\end{array}$ \\
\hline 1 & 1 & $\begin{array}{l}\text { Convective } \\
\text { disc. }\end{array}$ & 436.602565 & 436.602565 & 1969.239615 & 695700 & 274 & 274 & $\begin{array}{l}200 \\
\left(1 * 10^{12}\right)\end{array}$ \\
\hline 1 & $3 / 4$ & $\begin{array}{l}4 \mathrm{p} 6 \mathrm{n} \\
\text { disc. }\end{array}$ & 283.581685 & 286.551447 & 1508.068146 & 521775 & 154.125 & 157.37 & $\begin{array}{l}150(0.75 \\
\left.* 10^{12}\right)\end{array}$ \\
\hline 1 & $2 / 3$ & $\begin{array}{l}\text { Radiative } \\
\text { disc. }\end{array}$ & 234.100417 & 230.556106 & 1248 & 459162 & 119.3544 & 114.61 & $\begin{array}{l}132(0.66 \\
\left.* 10^{12}\right)\end{array}$ \\
\hline 1 & $1 / 2$ & $\begin{array}{l}4 \mathrm{p} 6 \mathrm{n} \\
\text { disc. }\end{array}$ & 154.362317 & 151.266563 & 945.454545 & 347850 & 68.5 & 65.78 & $\begin{array}{l}100 \quad(0.5 \\
\left.* 10^{12}\right)\end{array}$ \\
\hline 1 & $2 / 5$ & weak & 110.452683 & 108.233652 & 756.363636 & 278280 & 43.84 & 42.1 & $\begin{array}{l}80\left(0.4^{*}\right. \\
\left.10^{12}\right)\end{array}$ \\
\hline 1 & $1 / 4$ & $\begin{array}{l}\text { Outer } \\
\text { core } \\
\text { disc. }\end{array}$ & 54.575321 & 91.901023 & 1396 & 173925 & 17.125 & 48.56 & $\begin{array}{l}50 \quad(0.25 \\
\left.* 10^{12}\right)\end{array}$ \\
\hline 1 & $1 / 5$ & $\begin{array}{l}\text { Inner } \\
\text { core } \\
\text { disc. } \\
\mathrm{r}_{c}\end{array}=$ & 39.050921 & 74.602949 & 1437.401179 & 139140 & 10.96 & 40 & $\begin{array}{l}40(0.2 * \\
\left.10^{12}\right)\end{array}$ \\
\hline
\end{tabular}

Note that multiplying any discontinuity radius with inner core velocity $\mathrm{v}_{c}$ gives

Table 32: Gravitational profile of the primordial Sun

values proportional to $\mathrm{r} / \mathrm{R}$ ratio and gives integer gravity $\left(\mathrm{g}_{i}\right)$ for inner core and all layers above.

I have previously hypothesized that the Sun has inflated to a much

\begin{tabular}{|c|c|c|c|}
\hline $\begin{array}{l}\text { discontinuity } \\
\quad(\mathrm{r} / \mathrm{R})\end{array}$ & $\begin{array}{l}\text { space velocity } \\
\mathrm{v}_{s}\end{array}$ & $\begin{array}{l}\text { correlated } \\
\text { radius } \\
\left(10^{6} \mathrm{~km}\right)\end{array}$ & possible body correlation \\
\hline $\begin{array}{l}1 \\
3 / 4 \\
2 / 3 \\
1 / 2 \\
2 / 5 \\
1 / 5\end{array}$ & $\begin{array}{l}436.6 \mathrm{~km} / \mathrm{s} \\
286.6 \mathrm{~km} / \mathrm{s} \\
230.6 \mathrm{~km} / \mathrm{s} \\
151.3 \mathrm{~km} / \mathrm{s} \\
108.2 \mathrm{~km} / \mathrm{s} \\
74.6 \mathrm{~km} / \mathrm{s}\end{array}$ & $\begin{array}{l}436.6 \\
286.6 \\
230.6 \\
151.3 \\
108.2 \\
74.6\end{array}$ & $\begin{array}{r}\text { end of the main asteroid belt } \\
\text { beginning of the main asteroid belt } \\
\text { orbit of Mars (semi-major) } \\
\text { orbit of Earth (semi-major, aphelion) } \\
\text { orbit of Venus (semi-major) } \\
\text { orbit of Mercury (aphelion?) }\end{array}$ \\
\hline
\end{tabular}
larger radius before being compressed to current one. In the exchange of components of angular momentum, radius might have been exchanged for space (Keplerian) velocity, as shown in Table 33. 
Table 33: Possible initial radii of Sun's discontinuities and correlation with bodies

However, orbits may be correlated with arithmetic mean of $\mathrm{v}_{s}$ and $\mathrm{v}_{p}$. This gives much better results for the orbit of Mercury - 56.8*10 $0^{6} \mathrm{~km}$, agreeing with semi-major, rather than aphelion. Another possibility is entanglement with $\mathrm{v}_{p}$ instead of $\mathrm{v}_{s}$. In that case $1 / 4$ $\mathrm{R}$ discontinuity roughly agrees with the orbit of Mercury.

Remarkable correlations are found subtracting velocities between layers, as shown in Table \begin{tabular}{l|l|l|l} 
discontinuity & space velocity $\mathrm{v}_{s}$ & correlated & possible body correlation
\end{tabular}

\begin{tabular}{|c|c|c|c|}
\hline 18 & - & $\mathrm{km})$ & \\
\hline $\begin{array}{l}1-3 / 4 \\
1-2 / 3\end{array}$ & $\begin{array}{l}436.6-286.6 \\
436.6-230.6\end{array}$ & $\begin{array}{l}150 \\
206\end{array}$ & $\begin{array}{r}\text { orbit of Earth (semi-major) } \\
\text { orbit of Mars (perihelion) }\end{array}$ \\
\hline
\end{tabular}

34. $1-2 / 3$

$3 / 4-2 / 3$

$3 / 4-1 / 5$

$2 / 3-1 / 5$

$2 / 5-1 / 5$

$1 / 2-2 / 5$

\begin{tabular}{l|l}
$436.6-230.6$ & 206 \\
$286.6-230.6$ & 56
\end{tabular}

\begin{tabular}{l|l}
$286.6-39.1$ & 247.5
\end{tabular}

\begin{tabular}{l|l}
$230.6-74.6$ & 156
\end{tabular}

\begin{tabular}{l|l}
$230.6-74.6$ & 69.1 \\
108.2 & 69.1
\end{tabular}

\begin{tabular}{l|l}
$154.4-108.2$ & 46.2
\end{tabular}

orbit of Mars (perihelion)

orbit of Mercury (semi-major) orbit of Mars (aphelion)* orbit of Earth (aphelion) orbit of Mercury (aphelion)* orbit of Mercury (perihelion)*

Table 34: Alternative initial radii of Sun's discontinuities

* here, one of the velocities used in subtraction is $\mathrm{v}_{p}$, rather than $\mathrm{v}_{s}$

Entanglement with $\mathrm{v}_{p}$ suggests that Mercury and Mars were created before Venus and Earth, as hypothesized previously. Entanglement with both, $\mathrm{v}_{s}$ and $\mathrm{v}_{p}$, seems to be the cause of orbital eccentricity.

Difference between current surface gravity and $g_{i}$ is roughly equal to the sum of surface gravities of inner and outer planets:

$$
g-g_{i}=274-200=74 \frac{m}{s^{2}}
$$

thus, some entanglement might exist there too.

Below the gravitational minimum at inner core $\left(\mathrm{r}_{c}\right)$, quantization is 3-dimensional (positive) and gravity should be increasing until the next maximum:

$$
\begin{gathered}
g=n^{2} T \frac{\hbar_{1}}{r^{2}}, \\
\hbar_{1}=1.273239545 * 10^{12} \frac{\mathrm{m}^{3}}{\mathrm{~s}^{3}}
\end{gathered}
$$

\subsubsection{Current G model}

Unlike in space above the outer maximum, where gravity falls to zero effectively at infinity (due to next maximum being extremely far), below the maximum gravity falls to zero at finite distance due to compression of space.

With no inner maxima, the single point of zero gravity would be at the center, however, due to relativity, inner maxima must exist (each inner maximum must also be a relative outer maximum).

If the radius of the outer maximum of the Sun is the surface radius, gravity should thus be decreasing below the surface to the point where it is canceled by the [next] inner maximum. 


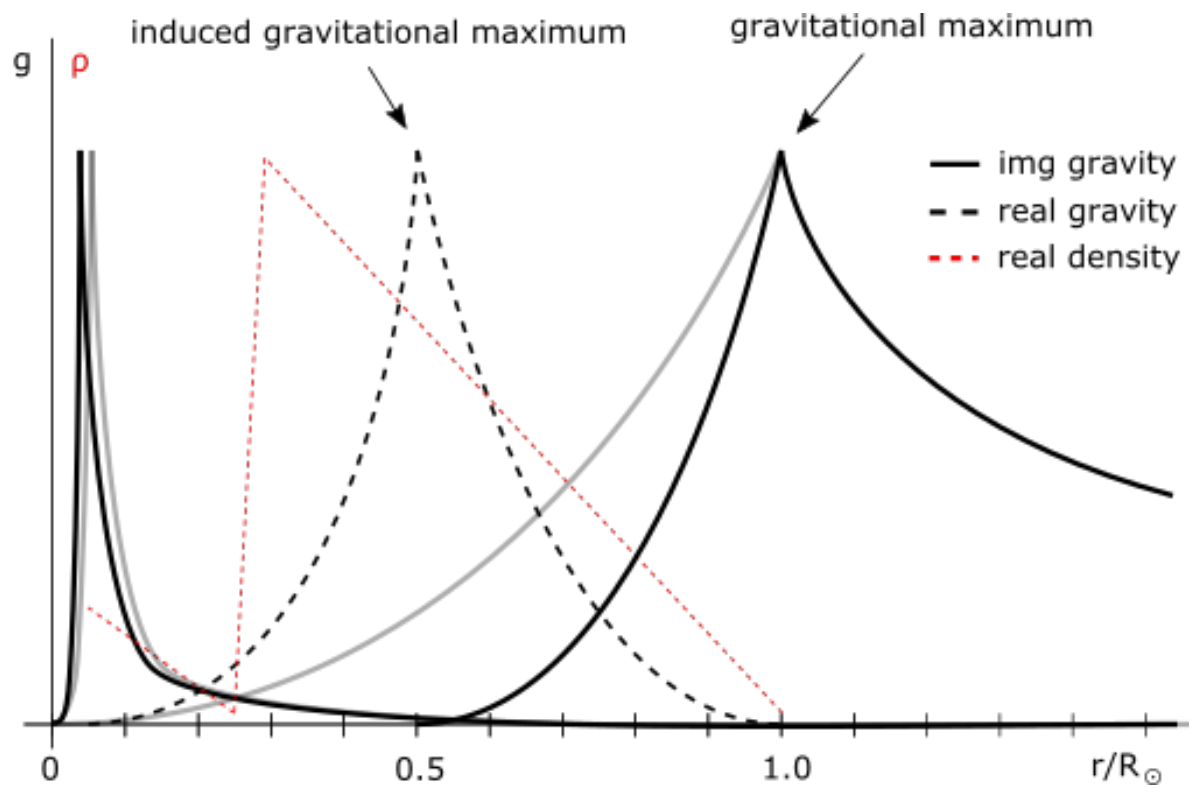

Figure 28: Gravity of the Sun

Without the inner maximum, any free-falling real mass would be concentrated around the surface maximum. With inner maxima, concentration of real mass begins at the center.

However, as each inner maximum has lower capacity than its outer maximum, greatest density of real mass will not be at the center. Once inner maximum is at full capacity, as real mass accumulates between the inner maximum and the outer maximum, its counteracting the gravity of the outer maximum.

In equilibrium thus, greatest density of real mass is not at the outer maximum, rather between the inner and outer maximum.

This is shown on Fig. 28. Here, dark matter gravity provided by [img] gravitational maxima is represented by solid black lines, while real gravity provided by real mass and its induced (effective) maximum is represented by dashed black lines. In case of outer maximum, gray line represents gravity with no real mass acquired (naked maximum), while for inner maximum, it represents the initial core maximum. Red dashed lines show linearly approximated density of real mass.

Note that Fig. 28 shows gravity in absolute values. Gravities of maxima cancel at multiple points inside the Sun. At these points, gravity is zero. Induced gravitational maximum should thus, in reality, have negative gravity relative to other maxima. 
From Fig. 28 one can extrapolate discontinuity candidates $\left(\mathrm{r} / \mathrm{R}_{\odot}\right): 0.0385$ $\approx 2 / 5 * 1 / 10,2 / 3 * 1 / 10$ (initial core maximum) $, 1 / 5,1 / 4,0.286,1 / 2,2 / 3,3 / 4$, 1.

Note that there should be two major charge radii inside the Sun, if the outer charge is located at tachocline, and charge radii are mirrored relative to the induced real maximum, other charge radius boundary should be at $2 / 5 \mathrm{R}_{\odot}$ (mirroring the $2 / 3 \mathrm{R}_{\odot}$ boundary).

In addition to these, there are other candidates, representing maximum deviation from these values - ie. discarding CMB relative kinetic energy, rest surface maximum is at $0.94 \mathrm{R}_{\odot}$.

\subsection{Energy replenishment}

Primary energy source of the Sun is, most likely, fusion.

Fuel for fusion must either be the real mass of the Sun (accumulated matter) or matter created through conversion of imaginary mass (dark gravitational potential) to real mass by some unknown mechanism.

In case of such conversion it would take tens of billions of years to spend all fuel.

However, this solution implies the Sun is eating itself and is highly unlikely.

The Sun is thus, most likely, burning its real mass which was accumulated during inflation of its maxima (whether through inflation of smaller maxima or acquisition of matter by increasing vacuum pressure).

When compared to other living beings, it would be reasonable to assume that Sun has a relatively constant real rest (constitutional) mass and an amount of fuel which is being cyclically replenished.

To determine how much fuel the Sun has left it is necessary to determine how much fuel it had at the beginning and the rate of fuel consumption.

Assuming fusion reaction $4 \mathrm{H}->\mathrm{He}$ (energy per reaction $\mathrm{E}_{r}=4.32 * 10^{-12}$ $\mathrm{J}$ ) and power output $\mathrm{P}$ of $3.8 * 10^{26} \mathrm{~J} / \mathrm{s}$, time needed to spend all fusion fuel is:

$$
\Delta t=\frac{m}{m_{p}} * \frac{E_{r}}{4} * \frac{1}{P} * N
$$

$$
\begin{array}{r}
\mathrm{m}=\text { available mass } \\
\mathrm{m}_{p}=\text { proton mass } \\
\mathrm{E}_{r}=\text { energy per reaction } \\
\mathrm{P}=\text { power output } \\
\mathrm{N}=\text { fraction of mass used in fusion }
\end{array}
$$


Since the Sun has two [major] maxima, fusion may be occurring at two places - in the core and above the core.

Gravitational mass of the surface maximum is known to be $1.988500 * 10^{30}$ $\mathrm{kg}$, while the gravitational mass of the core has been calculated here to be $2.951797 * 10^{27} \mathrm{~kg}$.

Assuming that the calculated mass is the mass of the maximum and therefor equal to the internal capacity for real mass, this capacity in equilibrium should be full and, due to mass loss (ie. through radiation), excess real mass must be constantly (cyclically) consumed as fuel.

Note that calculated mass implies such density of the core that temperature should be orders of magnitude higher than current assumptions, for thermonuclear fusion to occur.

If fusion is occurring in the core, most likely it is not thermonuclear.

It has also been hypothesized that the ratio of core mass and surface mass should be correlated with the ratio of mass between inner and outer planets.

Assuming that at the beginning of the core feeding cycle, these ratios are equal, fuel mass is the excess mass in the outer core corresponding to the ratio.

In case of thermonuclear fusion and with $2 / 3$ of mass consumed, time needed for the core to spend all fuel is:

$$
\begin{gathered}
\Delta t=\frac{m}{m_{p}} * \frac{E_{r}}{4} * \frac{1}{P} * N=\frac{8.90211033 * 10^{27} \mathrm{~kg}}{1.67265 * 10^{-27} \mathrm{~kg}} * \frac{4.32 * 10^{-12} \mathrm{~J}}{4} * \frac{1}{3.8 * 10^{26} \frac{\mathrm{J}}{\mathrm{s}}} * \frac{2}{3} \\
\Delta t=10084091956967735 \mathrm{~s}=319545591.5 \text { years }
\end{gathered}
$$

where $\mathrm{m}=8.90211033 * 10^{27} \mathrm{~kg}$ is the previously calculated initial mass of the core.

Assuming that, at the start of consumption cycle, imaginary mass (gravitational maximum) grows to initial mass radius $\left(0.286 \mathrm{R}_{\odot}\right)$ and decreases with energy loss, time left (assuming constant rate of consumption) before the next feeding cycle is then:

$$
\begin{gathered}
t=\left(2.951797 * 10^{27}-\frac{1}{3} 8.90211033 * 10^{27}\right) * \frac{3}{2} \frac{1}{8.90211033 * 10^{27}} \Delta t \\
t=-26461406017707 s=-838511.4 \text { years }
\end{gathered}
$$

Negative time may be interpreted as the next cycle being overdue (core spent all fuel $838 \mathrm{k}$ years ago and is currently burning constitutional mass), or, that more than $2 / 3$ of mass must be consumed in fusion.

In case $70 \%$ of mass may be spent:

$$
\Delta t=10588296554816122 \mathrm{~s}=335522871 \text { years }
$$




$$
t=1114734114271587 s=35323792.5 \text { years }
$$

However, as stated already, thermonuclear fusion in the core is unlikely. In case there is no fusion in the core at all, ruling out standard chemical reactions and radioactivity, the remaining possibility is heat generation through gravitational (Kelvin-Helmholtz) contraction:

$$
\begin{gathered}
\frac{d U_{r}}{d t}=\frac{-3 G M_{i}^{2}}{10 R_{i}{ }^{2}} \frac{d R}{d t} \\
\quad \mathrm{M}_{i}=\text { initial core mass }=8.90211033^{*} 10^{27} \mathrm{~kg} \\
\mathrm{R}_{i}=\text { initial core radius }=0.286 \mathrm{R}_{\odot}=198970200 \mathrm{~m}
\end{gathered}
$$

assuming logarithmic relationship between mass and radius contraction, the contraction may be approximated from the rate of Jupiter contraction:

$$
\begin{gathered}
\frac{d R}{d t}=\frac{10^{\frac{M_{i}}{M_{J}}}}{3^{\frac{R_{i}}{R_{J}}}} \frac{d R_{J}}{d t}=-7.29401291 * 10^{-8} \frac{\mathrm{m}}{\mathrm{s}} \\
\mathrm{M}_{J}=\text { Jupiter mass }=1.89819^{*} 10^{27} \mathrm{~kg} \\
\mathrm{R}_{J}=\text { Jupiter radius }=71492000 \mathrm{~m}
\end{gathered}
$$

giving energy radiation of:

$$
\frac{d U_{r}}{d t}=2.9233705 * 10^{21} \frac{\mathrm{J}}{\mathrm{s}}
$$

and time to spend all fuel:

$$
\begin{aligned}
\Delta t=\frac{3 G\left(M_{i}-M\right)^{2}}{10 R_{i}}\left(\frac{d U_{r}}{d t}\right)^{-1} & =1218751736351319 \mathrm{~s}=38619912 \text { years } \\
\mathrm{M} & =\text { current core mass }=2.951797^{*} 10^{27} \mathrm{~kg}
\end{aligned}
$$

From this one can calculate the core radius at the end of the cycle (all fuel spent):

$$
\begin{array}{r}
R=R_{i}-\Delta t \frac{d R}{d t}=R_{i}-\left(M_{i}-M\right)^{2} \frac{R_{i}}{M_{i}{ }^{2}}=0.158221 R_{\odot}=110074291 \mathrm{~m} \\
\mathrm{R}_{\odot}=\text { Sun surface radius }=695700000 \mathrm{~m}
\end{array}
$$

With current core radius at $0.2 \mathrm{R}_{\odot}$, amount of fuel left is:

$$
\frac{0.2-0.158221}{0.286-0.158221}=0.327 \approx \frac{1}{3}
$$


It is unlikely though that all fuel is spent during the cycle, total amount spent is most likely equal to $2 / 3$ (equivalent with fusion), in which case the cycle period is:

$$
\Delta t_{r e}=\frac{2}{3} \Delta t=25746608 \text { years }
$$

and the core is at the end of a cycle.

The obtained core cycle period agrees well with the hypothesized 2nd order cycle period of the Solar System $(\approx 26$ million years).

Since the 2nd order cycle period is also equal to periodicity of impacts and extinctions on Earth and other planets, all these Solar events are likely synchronized - once the core fuel is exhausted, additional fuel is provided by the outer half of the Sun at the same time equal quantity of its own fuel is replaced with mass from impactors.

Gravitational stress may even create wormholes through core/surface sunspots enabling direct consumption of impactor mass by the core.

Note that, with core radius oscillation, its time independent radius is obtained from the volumetric superposition of $0.2 \mathrm{R}_{\odot}$ and $0.286 \mathrm{R}_{\odot}$ cores:

$$
\begin{gathered}
\frac{4}{3} \pi R^{3}-\frac{4}{3} \pi R_{c}{ }^{3}=\frac{4}{3} \pi R_{i}{ }^{3}-\frac{4}{3} \pi R^{3} \\
R^{3}=\frac{R_{i}{ }^{3}+R_{c}{ }^{3}}{2}=\frac{\left(0.286^{3}+0.2^{3}\right) R_{\odot}{ }^{3}}{2} \\
R=\sqrt[3]{\frac{\left(0.286^{3}+0.2^{3}\right)}{2}} R_{\odot}=0.25 R_{\odot}=\frac{1}{4} R_{\odot}
\end{gathered}
$$

Such oscillation must be present on standard scale too - thus, all results obtained from measurements of nuclear observables may be understood as superpositions in time and/or space, however, in reality these are not constants, rather statistical mean state of changing phenomena.

Regardless of scale, no equally evolved (identical) phenomena can exist at two points in time, nor can they exist at multiple points in space. De-localization may seem possible through stretching of [a point in] space/time, however, this is fragmenting (quantizing) the phenomena and its space. Even if it remains strongly entangled, it is never, as a whole, at multiple points in space/time, although, with energy applied, de-localized space may collapse to one of the fragmented points.

Unlike the core, the outer part of the Sun is most likely powered by fusion.

However, it too must have constitutional mass and fuel mass fraction of real mass (excess mass).

Most likely, fuel mass is equal to the previously calculated kinetic energy 
(CMB relative) of the Sun. In that case, time to spend the fuel is:

$$
\begin{gathered}
\Delta t=\frac{m}{m_{p}} * \frac{E_{r}}{4} * \frac{1}{P} * N=\frac{1.18437729 * 10^{29} \mathrm{~kg}}{1.67265 * 10^{-27} \mathrm{~kg}} * \frac{4.32 * 10^{-12} \mathrm{~J}}{4} * \frac{1}{3.8 * 10^{26} \frac{\mathrm{J}}{\mathrm{s}}} * \frac{2}{3} \\
\Delta t=4.25 * 10^{9} \text { years }
\end{gathered}
$$

The value is in agreement with the hypothesized 1st order cycle and it is likely equal to previously calculated real age of Earth $\left(4.29 \pm 0.05 * 10^{9}\right.$ years), suggesting the Solar System is at the end of the 1st order cycle. Note that the calculated age is exactly $1 / 3$ of the obtained age of the observable universe in one class of measurements (Lensedquasars/Near) $-12.75 * 10^{9}$ years (also in agreement with more recent bTFR measurements[68]), supporting the cycling hypothesis (this would be the end of a 3rd cycle).

Gravitational stress of the 1st order must be order(s) of magnitude larger than that of the 2 nd order.

Likely, at the end of such cycle, Sun briefly looses some momentum (relative to CMB) with the spin change of the outer maximum. It falls into a lower energy level, closer to the galactic center. Afterwards, it starts expanding again consuming hydrogen fuel as it returns to the current state again.

Note that a reason for discrepancy in measurements of the age of the universe (Hubble constant) could be the same as in the case of the age of Earth. I have previously hypothesized cyclic time compression (evolution inflation, due to gravitational stress), with coupled periods of 1.512 and $\approx 26$ million years. With the next larger period being $\mathrm{T}_{u}=4.25 \mathrm{~Gy}$, its time compression should be:

$$
\Delta t_{c_{u}}=\frac{\Delta t_{c_{x}}}{T_{x}} T_{u}=\frac{24751.794 y}{1512000 y} 4.25 * 10^{9} y=69573495.04 \text { years }
$$

where $\Delta \mathrm{t}_{c_{x}}$ is the previously calculated compression of time with a single Tx (1512000 years) pulse.

Now one can calculate how much overestimated is the currently accepted age of the observable universe $\mathrm{T}_{i m g}=13.799 \pm 0.021 * 10^{9}$ years:

$\sigma_{T_{i m g}}=\left\lfloor\frac{T_{i m g}}{T_{u}}\right\rfloor\left[\Delta t_{c_{u}}+\left(\Delta T_{E_{i m g}}-T_{u}\right)\right]=1.07872048512 \pm 0.05 * 10^{9}$ years where $\Delta \mathrm{T}_{E_{i m g}}\left(4.54 \pm 0.05 * 10^{9}\right.$ years $)$ is the currently accepted age of Earth.

This gives for the real age of the universe:

$$
T=T_{i m g}-\sigma_{T_{i m g}}=12.72027951488 \pm 0.071 * 10^{9} \text { years }
$$


resolving the discrepancy.

Another interesting solution is obtained if the fuel amount is equal to real mass of the Sun calculated with the assumption of, across Solar System, invariant, real $\hbar_{m g}$ constant:

$$
m=\frac{\hbar_{m g}}{g}=\frac{6.968267285 * 10^{20} \mathrm{~N}}{274 \mathrm{~m} / \mathrm{s}^{2}}=2.543163243 * 10^{18} \mathrm{~kg}
$$

For $\mathrm{N}=2 / 3$ (here, the other $1 / 3$ would be the solar wind), time needed to spend this fuel is:

$$
\begin{gathered}
\Delta t=\frac{m}{m_{p}} * \frac{E_{r}}{4} * \frac{1}{P} * N=\frac{2.543163243 * 10^{18} \mathrm{~kg}}{1.67265 * 10^{-27} \mathrm{~kg}} * \frac{4.32 * 10^{-12} \mathrm{~J}}{4} * \frac{1}{3.8 * 10^{26} \frac{\mathrm{J}}{\mathrm{s}}} * \frac{2}{3} \\
\Delta t=\frac{2}{3} * 4321249.297 \mathrm{~s}=33.3 \text { days }
\end{gathered}
$$

For $\mathrm{N}=1 / 2$ :

$$
\Delta t=\frac{1}{2} * 4321249.297 \mathrm{~s}=25 \text { days }
$$

This solution is not plausible as it requires continuous hydrogen uptake from interstellar medium. While charged protons and electrons are absorbed at Sun's poles and could be combined to form hydrogen at the center (assuming the Sun is not ideally neutral and has gravitational holes at poles - at least periodically opened, although the charges could also be inefficiently transfered inside as electric current), energy bandwidth is not sufficient to power the Sun.

Interestingly, the solution (with $\mathrm{N}=2 / 3$ ) is close to the polar rotation period of the Sun $(\mathrm{N}=1 / 2$ gives equatorial period) where the uptake would happen.

However, although unlikely in a stable state, this is likely the feeding method in the previous hypothesis $\left(4.25 * 10^{9}\right.$ years cycle). Once the spin momentum collapses into a two-dimensional form, the Sun will be extremely charged. With an extremely strong non-homogeneous magnetic field it would be able to acquire required mass efficiently and quickly.

Differential rotation of the Sun could be a fossilized evidence of spin collapse, suggesting it breaks into multiple quanta in the form of concentric rings (oppositely charged rings must have anti-aligned spin to conserve the magnetic field).

Such fossil is perhaps more evident on Jupiter, where wind velocities are correlated with gravity. 
The extremely stable and static cyclones on Jupiter's poles indicate that it might have small gravitational holes open today.

However, if these are open, small gravitational gaps or indentations should also exist between layers associated with each ring quanta. Strong magnetic field and measurements of gravity do support this theory, although the indentations would have to be extremely small - if gravitational disturbances are not due to standard $\left(\mathrm{U}_{0}\right)$ scale matter, as currently interpreted (in which case they would be the fossil of the healing process).


Figure 29: Jupiter gravity disturbances and wind gradient ${ }^{69}$

The cells of all living species are regenerating on a periodic basis, for example, $1 / 3$ of hippocampal neurons in humans and mice is exchanged during the lifetime[70], thus, the cellular regeneration in the Sun should not be surprising, whether it is food or constitutional mass.

Capacity for real mass below the Sun's surface may be full, but all mass orbiting the Sun may be considered as its real mass.

However, it is obviously not fuel mass, rather constitutional or symbiotic mass.

The 3rd order period of the Solar System cycle may be related to this mass through the mass barycenter of the system.

I have previously calculated the neutral gravitational mass equivalent for the surface plasma at the equator which would make its angular velocity Keplerian.

The source for this energy may be the motion of the barycenter.

In any case, if one assumes that conversion between neutral and electromagnetic component of the general force of the Sun is also periodic and that 
such energy replaces fusion reactions in equivalent way, the period of recharge is:

$$
\begin{gathered}
\Delta t=\frac{m_{r e}}{m_{p}} * \frac{E_{r}}{4} * \frac{1}{P} * N=\frac{4.042341 * 10^{25} \mathrm{~kg}}{1.67265 * 10^{-27} \mathrm{~kg}} * \frac{4.32 * 10^{-12} \mathrm{~J}}{4} * \frac{1}{3.8 * 10^{26} \frac{\mathrm{J}}{\mathrm{s}}} * \frac{2}{3} \\
\Delta t=45790644230537 \mathrm{~s}=1451018 \text { years }
\end{gathered}
$$

and it is in good agreement with the hypothesized 3rd cycle period (a fraction of mass $\mathrm{N}=0.6946847$ would yield the hypothesized value - 1512000 years).

In comparison with living beings, one might notice a problem of exhausted fuel - what happens with the ash from fusion reactions (end products of fusion)?

There are couple of solutions:

1. the ash is ejected periodically,

2. the ash forms the constitutional mass.

Time compression at the end of Solar System cycles implies gravitational stress of Solar System maxima.

While the 2nd hypothesis might be plausible during initial formation of the Sun, at least at the end of one of the cycles some mass must be ejected out from the Sun.

It certainly seems easier than in case of planets, as unlike the planets, the Sun does not have a solid [real] mantle to block the explosion (the mantle of the Sun are the terrestrial planets, however, they are in collapsed form with plenty of space in between).

The ash content depends on the cycle period, being mostly Helium in smaller cycles but with heavier elements formed in explosions at the end of larger cycles.

A full collapse of the maximum is the collapse of a 3-dimensional spherical neutral form into 2-dimensional charged form. Since the surface maximum of the Sun is entangled with Mars' maximum, at the time of collapse, two ring maxima are aligned and the ejection of ash is not isotropic, rather targeting Mars.

At that point, both the Sun and Mars have a significant (extreme) magnetic field generated by charged maxima so Mars would likely attract ferromagnetic/charged ejecta from the Sun.

The evidence for this is the Fe covered surface of Mars.

Note that the collapse involves the change of spin of the maxima. First, the holes are opening on the poles of the spherical Sun maximum while the axial tilt starts increasing, the poles of the Sun and Mars are only briefly fully aligned before the equilibrium of stable spin states is reached. Thus, most mass is ejected in the first and last moments of the spin change, through the equilibrium poles - out of the Solar System. 
Note that the magnetic field is weakest at these times, as it increases, the momentum of particles is curved and aimed at Mars.

\subsection{As a living organ[ism]}

Considering the energy output (metabolic rate) of $\mathrm{P}=3.8 * 10^{26} \mathrm{~W}$, the standard relation between metabolic rate and mass[71]:

$$
\frac{P}{0.0484259259 \frac{\text { day*W }}{\text { kcal }}}=70 * M^{\alpha}
$$

gives 0.86 for the $\alpha$ exponent $\left(\mathrm{M}=\right.$ total mass of the $\operatorname{sun}=1988500 * 10^{24}$ $\mathrm{kg}$ ). For a mammalian organ this would be between a kidney and a liver[72], suggesting an embryonic stem cell in the process of differentiation.

\section{Metabolism of Earth}

Transfer of energy in wild flora and fauna is generally balanced both horizontally and vertically.

Vertical transfer of energy is a part of metabolism but changes in horizontal current affect the vertical transfer too (and vice versa).

Humans dominate in both horizontal (surface to surface) and vertical (Sun Earth interior) energy distribution and transformation, disrupting the harmonics of life.

Horizontal effect is the increasing number of individuals at the cost of decreasing number and diversity of other species, while vertically it is the unsustainable exploitation of radiated and stored resources of the Sun/Earth ecosystem.

Thus, one may interpret humans as the metabolism energy carrier particles, in a limited domain.

With a human population $\mathrm{N}$ of $7.674 * 10^{9}$, average mass $\mathrm{m}$ of $62 \mathrm{~kg}$, and average lifetime $\Delta \mathrm{t}$ of 72.6 years (data for year 2019, except mass - 2012):

$$
\begin{gathered}
P=\frac{N * m * c^{2}}{\Delta t}=\frac{7.674 * 10^{9} * 62 *\left(2.99792458 * 10^{8}\right)^{2}}{72.6 * 365.25 * 24 * 60 * 60}=1.86644116 * 10^{19} \mathrm{~W} \\
\frac{P}{0.0484259259 \frac{\mathrm{day} * \mathrm{~W}}{\mathrm{kcal}}}=70 * M^{\alpha}=3.8542188 * 10^{20} \frac{\mathrm{kcal}}{\mathrm{day}}
\end{gathered}
$$

where $\mathrm{M}$ is the mass of Earth $\left(5.9723 * 10^{24} \mathrm{~kg}\right)$.

This gives a value of 0.756 for $\alpha$ exponent, in agreement with Kleiber's law.

However, in case of organ interpretation, the exponent suggests a superposition of a brain and a kidney. 
Note that Earth has kidney [precursor] equivalents on surface.

In order for this superposition to differentiate into the brain, the exponent would have to reduce to 0.7 .

There are several ways to achieve that (sorted by probability, from highest to lowest):

1. increasing human lifetime $(\approx 25$ times $)$ to 1813 years,

2. reducing population ( $\approx 25$ times) to 307243423 ,

3. reducing mass ( $\approx 25$ times),

4. increasing Earth's mass $\approx 100$ times ( $\approx$ mass of Saturn).

If humans are indeed precursor proteins of neuron proteins of Earth, as carriers of energy of its brain metabolism, I would expect the solution to be a superposition of the above.

However, if Earth has a heart equivalent (core), most likely it also has a kidney equivalent. Thus, I would expect the population to differentiate into proteins of varying function.

I, strive for neutrality - the equal, balanced usage of all parts of my universe. I am aware though, that this is an unreachable singularity, but it is the journey that makes one alive - for without it there would be no senses, for a sense of reason, and a reason for existence.

\subsection{Nature of human cells}

Dominance of lifeforms changes over time. At present time, homo species occupies and controls most of the surface of the planet. Human population is rising and thriving at the expense of other species.

While the dominion of species may be related to precursor nature of vital organism components, its behavior can be corrupted, so cultivation of new proteins becomes evolution of disease rather of something integral for survival.

While it is not questionable whether human species is a disease for the planet, it is questionable whether this is fatal or rather a normal part of evolution of healthy cells and proteins with self-correcting mechanisms.

Dividing the total surface area of Earth $\left(\mathrm{R}=6371 * 10^{3} \mathrm{~m}\right)$ with the number of people, one gets the maximum size of the cell:

$$
A=\frac{4 \pi R^{2}}{7.7 * 10^{9}}=66242.13921 \mathrm{~m}^{2}
$$

Radius of space per person is:

$$
r=\sqrt{\frac{A}{\pi}}=145.2085665 \mathrm{~m}
$$


If the radius of a human occupied cell of Earth is the mean free path $r$, the radius of a cell equivalent in human body of average diameter (height) $\mathrm{h}=1.7$ $m$ is:

$$
r_{c}=\frac{r}{R} \frac{h}{2}=19.373298 * 10^{-6} m=19.373298 \mu m
$$

If one calculates using landmass only (people don't naturally live on water):

$$
\begin{gathered}
A=\frac{1.4894 * 10^{14} \mathrm{~m}^{2}}{7.7 * 10^{9}}=19342.85714 \mathrm{~m}^{2} \\
r=\sqrt{\frac{A}{\pi}}=78.46669775 \mathrm{~m} \\
r_{c}=\frac{r}{R} \frac{h}{2}=10.46879502 * 10^{-6} \mathrm{~m}=10.46879502 \mu \mathrm{m}
\end{gathered}
$$

Taking into account space used by wild flora and fauna:

$$
\begin{gathered}
r=\frac{1}{2} \sqrt{A}=69.53930029 \mathrm{~m} \\
r_{c}=\frac{r}{R} \frac{h}{2}=9.277728025 * 10^{-6} \mathrm{~m}=9.277728025 \mu \mathrm{m}
\end{gathered}
$$

This is in the range of a typical cancer cell. It is, of course, in the range of healthy cells too, but human cells are far from healthy.

It might seem that the radius $\mathrm{r}\left(\mathrm{r}_{c}\right)$ changes with population, but this is not the case - if human space decreases, the space of wild flora and fauna increases and vice versa, thus it generally evolves weakly, remaining almost constant.

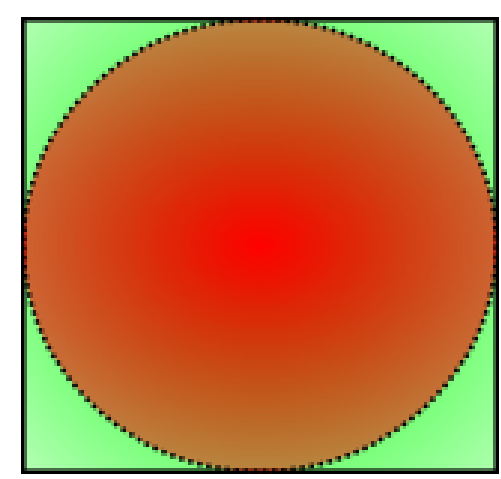

Figure 30: Homo.beta cell

Fig. 30 shows the unit of space on Earth's surface, circled space (red) is occupied by a human and domesticated flora and fauna, other (green) by wild flora and fauna. 


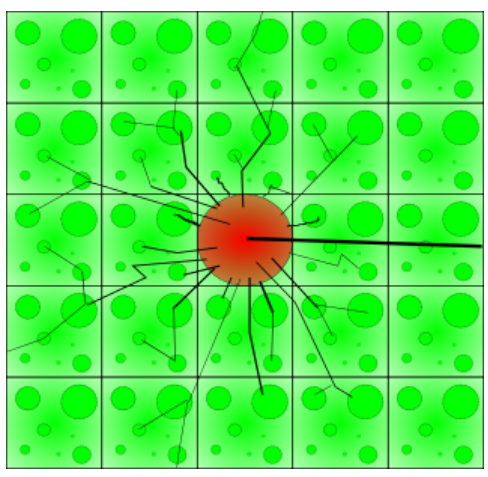

Figure 31: Normal cells

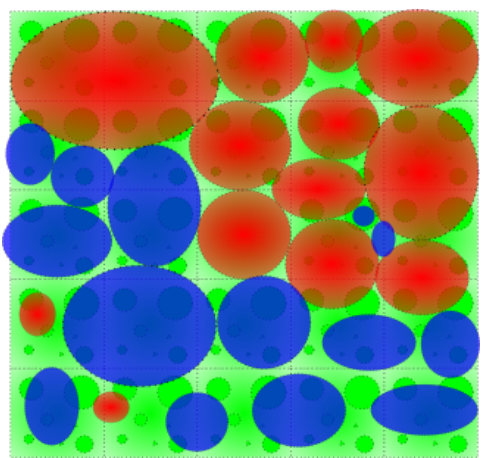

Figure 32: Cancer cells 
Fig. 31 shows the normal (healthy) unit of space on Earth. Red is a cell of homo.sapiens (Earth's neuron cell), black lines are spiritual connections (synapses).

Fig. 32 shows the cancerous (current) unit of space on Earth. Blue and red are polarized human (cancer) cells.



Figure 33: Dead space

Fig. 33 shows the outcome - death.

Carbon footprint is not the issue. It is just a side-effect of the real issue - nature of the human footprint.

Cancer cell contains the individuals (proteins) and space affected by cancerous population, but one can even calculate the role of a human in the cancer cell:

$$
\lambda=\frac{h}{2} \frac{1}{r} r_{c}=\frac{h}{2} \frac{1}{R} \frac{h}{2}=\frac{h^{2}}{4 R}=1.134044891 * 10^{-7} \mathrm{~m}
$$

This is in the range of a TGF- $\beta$ protein, a key player in cancer development.

Confirmation of this comes from recent studies[73], revealing human nature of TGF- $\beta$ :

"And while it may be difficult to imagine a protein with two dramatically different faces, it may be even more difficult to contemplate cancer cells exhibiting traits, such as cunning and deception. But the research underway at the University of Basel, and collaborating laboratories, has revealed that TGF- $\beta$ not only is a two-faced protein, it also is one that seems almost Machiavellian in its activities. "[74]

Cancerous TGF- $\beta$ suppresses the immune response and prevents old cell$\mathrm{s} /$ proteins from dying (regenerating). Humanity is, at the time of this writing, expressing this cancerous behavior on many levels: 
- through treatment of diseases (including cancer) humanity is suppressing the immune system of Earth,

- forcing human life at all costs and treating death (as a disease) - instead of letting cells (and proteins - people/animals) die as programmed so they can regenerate,

- treating Earth and other life forms (and, generally, even people) as resources - instead of living in a sustainable symbiotic relationship,

- creating and living in centralized, stressful environments, promoting inequality in wealth and health,

- denying the truth.

Earth's cells are not fuel cells, they are living cells.

The average cell cycle period of eukaryotic cell is $\mathrm{T}_{0}=14.5$ hours, scaled to Earth size, it is:

$$
T_{1}=\sqrt{T_{0} * T_{x}}=50 \text { years }
$$

where $\mathrm{T}_{x}$ is the period of $3 \mathrm{rd}$ order existence cycle of Earth $\left(1.512 * 10^{6}\right.$ years $)$.

\section{The cycle of life and death}

An atom or a planetary system consists of relatively massive matter and relatively empty space of gravitational wells.

The energy of this space is in its vacuum proportional to its spin momentum and characterized by electric and magnetic permeability of polarized quanta of certain scale.

Gravitational wells (souls) are not intrinsically coupled with matter - otherwise, there would be no death.

All souls thus oscillate between different bodies.

This oscillation can be vertical (between different scales) or horizontal (between species of the same element, such as carbon), although even horizontal oscillation includes a temporary scale inflation/deflation between stable states.

Species in horizontal oscillation have comparable lifetimes so gravitational collapse generally indicates a permanent decoupling of particular soul/matter pair (death).

Primary (prevalent) oscillation type depends on pressure/temperature of the environment.

Man's desire to extend his dying baffles all of common sense. Why would one not want to leave the aging body and start anew? It seems, 
it is in nature of polarized to keep patching the patches of the aging systems instead of letting things die and recycle in peaceful honor.

It is alas, the fear of death, unjustly implanted into the seeds of man by man that makes him a zombie, sad and cancerous, even at times of abundant life.

\section{Dark energy}

If planetary systems are [condensed] atoms, observable universe becomes a gas of extremely low density. Dark energy, if it exists, is thus simply the energy of gas expansion due to scaled pressure/temperature change. Such expansion requires exponentially increasing amount of energy, cannot continue forever, and at this state of the observable universe, it is more likely to be decelerating, rather than accelerating.

Galaxies are simply quantum vortices.

Black holes and other gravitational wells of $\mathrm{U}_{1}$ scale can be understood then as vacuum quanta, increasing in strength with expansion and causing contraction of matter, with stretched space between them creating (inflating) new gravitational wells between galaxies. This is the reason of exponential growth of energy requirement.

The expansion of the universe and its metric has been questioned before[75] and results are consistent with a non-expanding, Euclidean universe, although the increase in redshift with distance was not explained.

Recent evidence shows that the expansion of the observable universe is not accelerating[76] and the redshift previously used as evidence for acceleration should be attributed to local "bulk flow" instead.

The fact that photon has a mass and the existence of energy absorbers of lower scale explains the redshift increase with distance. It should be noted also that, due to carrier mass, forces of nature change over time, splitting in strong evolution events and evolving slowly during weak evolution.

\section{Stability of elements}

Structure of $U_{0}$ elements is entangled with the configuration of $U_{1}$ universe. This also makes the stability of isotopes dependent on this configuration.

The stability curve and decay rates of individual isotopes thus change strongly in transition from one cycle state to another, but also oscillate during state lifetime.

Stable isotopes are concentrated along this curve:

$$
\begin{gathered}
N(P, t)=\left\lfloor P *\left[1+\left(\frac{N_{\max }}{P_{\max }}-1\right) * \frac{P}{P_{\max }}\right]+\sigma_{T}\right\rceil \\
\sigma_{T}=\left[-\left(C_{1} * C_{2}\right) *\left(\frac{C_{2}}{C_{1}}-1\right)+\left(C_{2}-C_{1}\right) * \frac{t}{\Delta_{t}} *\left(C_{1}+C_{2}\right)\right] * \frac{P}{P_{\max }}
\end{gathered}
$$




$$
\sigma_{T}=\left[\left(C_{1} * C_{2}-C_{2}{ }^{2}\right)+\left(C_{2}^{2}-C_{1}{ }^{2}\right) * \frac{t}{\Delta_{t}}\right] * \frac{P}{P_{\max }}
$$

where $\mathrm{N}=\mathrm{N}_{0}$ is the number of neutrons, $\mathrm{P}=\mathrm{P}_{0}=\mathrm{Z}$ is the number of protons of the isotope and $\mathrm{P}_{\max }$ is the maximum number of protons for a stable element (for the Solar and equivalent systems, $\mathrm{P}_{\max }=82$, corresponding to $\mathrm{Pb}-$ lead). $\sigma_{T}$ is the small shift in value of $\mathrm{N}$ due to weak evolution through state lifetime $\left(\Delta_{t}\right)$.

$$
\begin{gathered}
\frac{P_{\max }}{N_{\max }}=\frac{N_{1}}{P_{1}} \\
P_{P_{\max } / N_{\max }}=\left\lfloor E H_{N_{1} / P_{1}}\left(P_{s}, N_{P_{\max } / N_{\max }}\right)\right\rceil
\end{gathered}
$$

where $\mathrm{N}_{1}$ is the number of neutrons and $\mathrm{P}_{1}$ the number of protons of the parent system - $\mathrm{U}_{1}$.

$\mathrm{P}_{s}$ is the atomic number (number of protons) of the most stable element element with maximum number of stable isotopes.

$\mathrm{P}_{P_{\max } / N_{\max }}$ is the atomic number of the element lying on the $\mathrm{N}(\mathrm{P}, \mathrm{t})$ curve with $\mathrm{P} / \mathrm{N}$ ratio equal to $\mathrm{P}_{\max } / \mathrm{N}_{\max }$.

For the Solar System, in state 6p4n:

$$
\begin{gathered}
\Delta_{t}=1.51 * 10^{6} \text { years } \\
\frac{P_{\max }}{N_{\max }}=\frac{2}{3} \\
P_{2 / 3}=\left\lfloor E H_{4 / 6}\left(P_{s}, N_{2 / 3}\right)\right\rceil \\
C_{1}=2, C_{2}=3
\end{gathered}
$$

Note that the constants $\mathrm{C}_{1}$ and $\mathrm{C}_{2}$ are the same as those determined in chapter "Earth, as a living organ[ism] - Age and 3rd order period - Speed of time".

Fig. 34 shows all stable isotopes of the Solar System (green) and the N(P,t) curve (black).

Note the following:

- for $\mathrm{t}>1495840$ years $\left(\mathrm{t} \approx \Delta_{t}\right)$, the isotope lying on the curve with $\mathrm{P} / \mathrm{N}$ ratio exactly equal to $2 / 3$ is $\mathrm{Pt}-195$ (Platinum, $\mathrm{P}=78$ ). The placement of other Platinum isotopes is symmetric relative to the curve, 


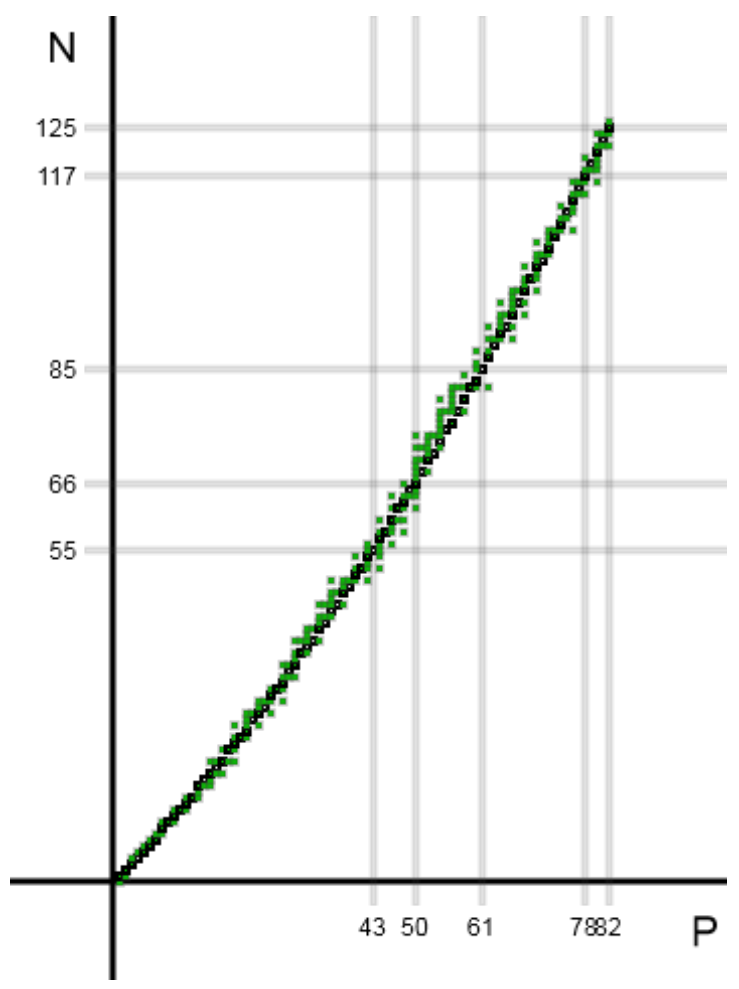

Figure 34: Stable isotopes of the Solar System in state 6p4n at t $>1495840$ years 
- for $\sigma_{T}=0\left(\mathrm{t}=3 / 5 \Delta_{t}\right)$, the $\mathrm{P}_{2 / 3}$ isotope is $\mathrm{Pb}-205$ (Lead, $\left.\mathrm{P}=82\right)$. At $\mathrm{t}$ $=3 / 5 \Delta_{t}$ this was a stable isotope. $1 / 3$ of other stable isotopes are above the curve, $2 / 3$ below,

- for $\mathrm{t}=4 / 5 \Delta_{t}$ the $\mathrm{P}_{2 / 3}$ isotope is Hg-200 (Mercury, $\mathrm{P}=80$ ). $1 / 3$ of other stable isotopes are above the curve, $2 / 3$ below,

- the ratio of horizontal to vertical distance between Lead-205 and Platinum195 is $(82-78) /(123-117)=4 / 6=2 / 3$,

- the ratio of horizontal to vertical distance between Lead-205 and Hg-200 is $(80-78) /(120-117)=2 / 3$,

- at $\mathrm{t} \approx \Delta_{t}$, Tin $(\mathrm{Sn}, \mathrm{P}=50)$ has the highest number of stable isotopes (10). Tin isotope lying on the curve is Sn-116 (50 protons, 66 neutrons). $2 / 3$ of other stable Tin isotopes is above the curve, $1 / 3$ is below,

- at $\mathrm{t} \approx \Delta_{t}$, the only elements without stable isotopes are Tc (Technetium, $\mathrm{P}=43$ ) and $\mathrm{Pm}$ (Promethium, $\mathrm{P}=61$ ). The isotopes lying on the curve are Tc-98 and Pm-146. Vertical distance from Sn-116 to Tc-98 is equal to horizontal distance from Sn-116 to Pm-146.

\section{Electric gravity}

Electric force is a polarized component of the general force.

Inside the atom, force field between negative and positive charges is neutralized and electro-magnetic potential may be exchanged with gravitational potential.

Thus, a Hill sphere radius $\left(\mathrm{r}_{H}\right)$ of an atom should be correlated with its charge radius.

$$
r_{H}=R \sqrt[3]{\frac{m}{3 M}}
$$

This gives, for Carbon-12 atom with nucleus mass $\mathrm{m}=1.992646883 * 10^{-26} \mathrm{~kg}$ inside the gravity field of Earth at $\mathrm{R}=6371 \mathrm{~km}$ (surface):

$$
r_{H}=66 * 10^{-12} \mathrm{~m}=66 \mathrm{pm}
$$

This is in agreement with experimentally obtained radius of $70 \mathrm{pm}( \pm 5 \mathrm{pm})$. Calculation for other elements of the periodic table yields similar results.

Note that Hill radius is different for different isotopes of the same element while experimentally obtained atomic radii are charge radii and thus independent of the number of neutrons (radius represents the orbit of the outermost electron). In example, for Carbon-14 the obtained value is $69.5^{*} 10^{-12} \mathrm{~m}$, and even closer to $70 \mathrm{pm}$ if one calculates using equatorial radius of Earth instead of mean volumetric (a possible indicator that the Solar System soul was a part of a ${ }^{14}(\mathrm{C}-\mathrm{N}-\mathrm{O})$ cycle in previous incarnation $)$. 

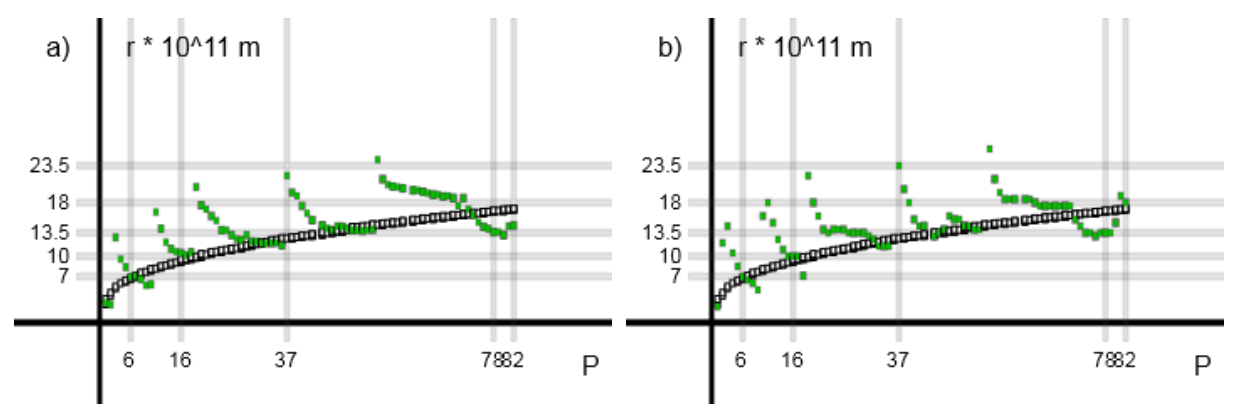

Figure 35: Calculated Hill sphere and measured radius for stable isotopes: a) data from 2008. b) data from 1964.

Fig. 35 shows experimentally obtained radius (green) and calculated Hill sphere at $\mathrm{R}=6371 \mathrm{~km}$ (black) for all stable isotopes. Evidently, radii are not only correlated but values of covalent radii oscillate around the Hill radii, confirming the entanglement of $\mathrm{U}_{0}$ and $\mathrm{U}_{1}$.

Comparing data from 1964.[77] and 2008.[78] shows a compression of radii and convergence to Hill radii - such changes are expected in CR (no constants) and these should be accelerating as the Solar System approaches the end of the current state $(6 \mathrm{p} 4 \mathrm{n})$.

In the intermediate state (5p5n) charges may be completely neutralized, and the radii of all elements may converge to Hill radius.
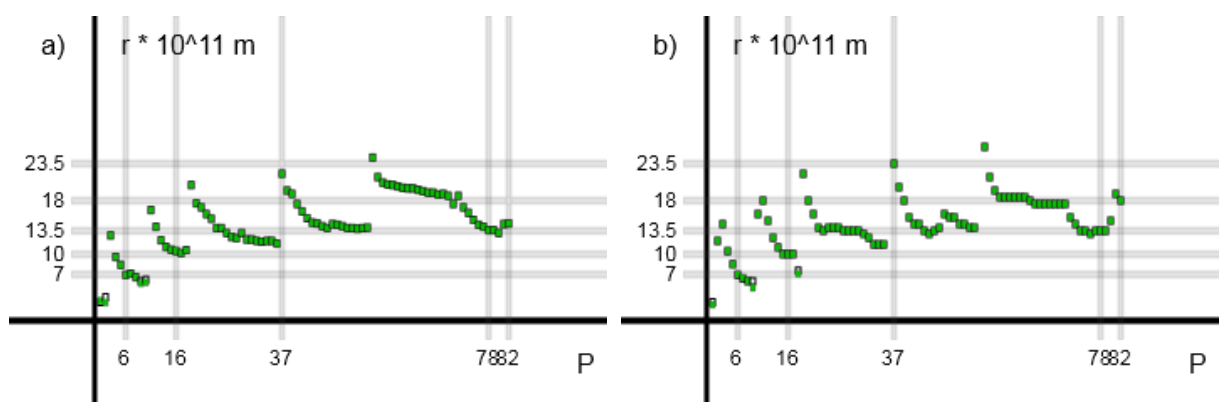

Figure 36: Calculated Hill sphere (adjusted) and measured radius for stable isotopes: a) data from 2008. b) data from 1964.

Fig. 36 shows the experimentally obtained radius (green) and calculated Hill sphere at $\mathrm{R}=6371 \mathrm{~km}$ (black) for isotopes with neutron number adjusted to match the charge radius.

In calculations above, atomic mass has been quantized by $\mathrm{u}=1.66053907 *$ $10^{-27} \mathrm{~kg}$ (atomic mass constant) with integer number of protons $\mathrm{P}$ and neutrons $\mathrm{N}\left[\mathrm{m}=(\mathrm{P}+\mathrm{N})^{*} \mathrm{u}\right]$ so Hill radii are quantized too. The overlap of Hill radii with charge radii in Fig. 36 shows that charge radius is quantized too (there is a number of neutrons $\mathrm{N}$ for which the Hill radius will match the charge radius). 


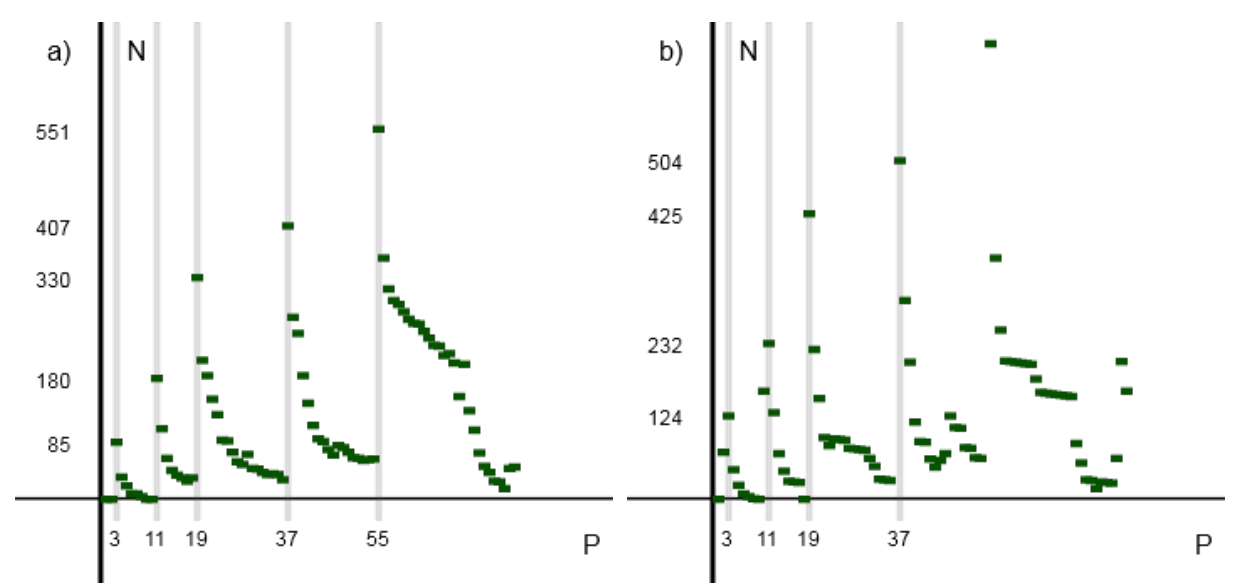

Figure 37: Isotopes used in Fig. 36 calculation: a) data from 2008. b) data from 1964.

Fig. 37 shows the number of neutrons $\mathrm{N}$ used with each element to obtain Hill radius equal to charge radius.

From above figures it is obvious that elements (atoms) are grouped into shells the same way as electrons are grouped in atoms. Grouping is shown in

\begin{tabular}{c|c|c|c|r}
$\mathrm{n}$ & shell (alt shell) & entanglement & elements & total elements $=2 \mathrm{n}^{2}$ \\
\hline 1 & $\mathrm{~K}$ & - & $1-2(\mathrm{H}-\mathrm{He})$ & 2 \\
2 & $\mathrm{~L}$ & $\mathrm{Q}$ & $3-10(\mathrm{Li}-\mathrm{Ne})$ & 8 \\
2 & $\mathrm{~L}(\mathrm{Q})$ & $\mathrm{L}$ & $11-18(\mathrm{Na}-\mathrm{Ar})$ & 8 \\
3 & $\mathrm{M}$ & $\mathrm{P}$ & $19-36(\mathrm{~K}-\mathrm{Kr})$ & 18 \\
3 & $\mathrm{M}(\mathrm{P})$ & $\mathrm{M}$ & $37-54(\mathrm{Rb}-\mathrm{Xe})$ & 18 \\
4 & $\mathrm{~N}$ & $\mathrm{O}$ & $55-86(\mathrm{Cs}-\mathrm{Rn})$ & 32 \\
4 & $\mathrm{~N}(\mathrm{O})$ & $\mathrm{N}$ & $87-118(\mathrm{Fr}-\mathrm{Og})$ & 32
\end{tabular}

Table 35: Grouping of elements

Table 35. There are two possibilities - either the shells L, M and N are doubled or the grouping is reflected after the $\mathrm{N}$ shell, so shells $\mathrm{O}, \mathrm{P}$ and $\mathrm{Q}$ contain the same number of elements such as shells N, M and L, respectively. Note that in case of alternative $(\mathrm{Og})$ grouping, no elements beyond $\mathrm{Og}$ are theoretically possible - otherwise another shell would be present between He and Li.

Gravitational constant $\mathrm{G}$ is not dimensionless and therefor not invariant to vertical scale transformation.

On the standard atom scale $\mathrm{U}_{0}$, gravitational constant for a completely neutralized general force can be derived from previously obtained orbital momentum of the Carbon-10 outermost electron:

$$
\frac{m v^{2}}{r}=G \frac{M m}{r^{2}}
$$




$$
\begin{gathered}
v^{2}=G \frac{M}{r} \\
M=\frac{\text { Sun mass }}{\text { Neptune mass }} * m \\
G=G_{0}=v^{2} \frac{r}{M}=1.234879253 * 10^{27} \frac{\mathrm{m}^{3}}{\mathrm{kgs}^{2}} \\
\mathrm{v}=\mathrm{v}_{U_{0}}=5.585837356 * 10^{5} \mathrm{~m} / \mathrm{s} \\
\mathrm{r}=\mathrm{r}_{U_{0}}=70^{-12} \mathrm{~m}
\end{gathered}
$$

where $\mathrm{m}, \mathrm{v}, \mathrm{r}$ are components of the outermost electron orbital momentum (mass, velocity, radius).

If one now, equalizes electric with gravitational force (for photon/graviton $\mathrm{m}>0$ - Yukawa, Proca[79]):

$$
k_{0} Q^{2}\left(\frac{1}{r^{2}}+\frac{\mu_{\gamma}}{r}\right) e^{\left(-\mu_{\gamma} r\right)}=G_{0} m^{2}\left(\frac{1}{r^{2}}+\frac{\mu_{n}}{r}\right) e^{\left(-\mu_{n} r\right)}
$$

discarding $\mu / \mathrm{r}$ factors due to being practically equal and equal to 0 on both sides (expecting large $r$ ):

$$
\begin{gathered}
\mu_{n}-\mu_{\gamma}=\frac{1}{r} \ln \left(\frac{G_{0} m^{2}}{k_{0} Q^{2}}\right) \\
\frac{M_{n} c_{n}}{\hbar_{n}}-\frac{M_{\gamma} c_{\gamma}}{\hbar_{\gamma}}=\frac{1}{r} \ln \left(\frac{G_{0} m}{k_{0} Q}\right) \\
\frac{c_{n}}{\hbar_{n}}=\frac{c_{\gamma}}{\hbar_{\gamma}}=\frac{c}{\hbar} \\
M_{n}-M_{\gamma}=\frac{\hbar}{c} \frac{1}{r} \ln \left(\frac{G_{0} m^{2}}{k_{0} Q^{2}}\right) \\
\hbar=\text { reduced Planck's constant }=1.054573^{*} 10^{-34} \mathrm{Js} \\
\mathrm{c}=2.99792458 * 10^{8} \mathrm{~m} / \mathrm{s} \\
\mathrm{k}_{0}=\text { Coulomb constant }=8.9875517873681764 * 10^{9} \mathrm{Nm}^{2} / \mathrm{C}^{2} \\
\mathrm{Q}=\text { electron charge }=1.60217733^{*} 10^{-19} \mathrm{C} \\
\mathrm{M}_{\gamma}=\text { photon mass } \\
\mathrm{M}_{n}=\mathrm{U}_{0} \text { graviton mass }
\end{gathered}
$$

Using previously obtained photon mass $\mathrm{M}_{\gamma}=2 * 9.10938356 * 10^{-73} \mathrm{~kg}$ and carbon graviton neutrino mass $\mathrm{M}_{n}=2 * 1.663337576 * 10^{-68} \mathrm{~kg}$, this gives:

$$
r=1.3032821975 * 10^{26} \mathrm{~m}
$$

as the distance in space when two forces become equal. 
Note that, with current arrangement, obtained $\mathrm{r}$ is actually negative. Positive $\mathrm{r}$ is obtained if $\mathrm{M}_{\gamma}$ and $\mathrm{M}_{n}$ exchange masses.

Graviton neutrino is bosonic (half-neutrino/anti half-neutrino pair $=\mathrm{e}-$ neutrino/anti e-neutrino pair) differing from photon only in charge/mass ratio, so the exchange is not impossible, rather expectable.

As shown previously, components of general force, charge and mass are exchangeable through inflation/deflation of momentum components (even in neutral particles, the amount of gravitational mass can increase at the expense of charge mass, with particle remaining neutral).

Nature of the force thus has to oscillate over distance.

Taking into account error margins, obtained distance is equal to the radius of observable universe, assuming currently accepted [img] age $\left(13.799 * 10^{9}\right.$ years $)$, constant speed of light and flat space:

$r=c \Delta t=2.99792458 * 10^{8} * 13.799 * 10^{9} * 365.25 * 24 * 60 * 60=1.305 * 10^{26} \mathrm{~m}$

The fact that obtained distance is equal to the radius of observable universe is not a coincidence.

For an inflation at the speed of light, for standard particles, distance in space is distance in time so this may be interpreted as the time when both forces (carrier masses) were equal, after which point one particle started loosing mass while the other was gaining mass.

This is expected with the exchange of one potential for the other.

Note that previously obtained real age of observable universe $(12.75 *$ $10^{9}$ years) implies inflation was at times faster than current $\mathrm{c}$ which, for the same radius, implies the $\mathrm{c}$ in flat space was also higher at these times. In expanding vacuum - with decreasing density, speed of light must be proportional or inversely proportional to speed of inflation wherever the density of space is affected.

In the past the observable universe did expand, but geometry deformation was localized (quantized, gravitational wells being the quanta of vacuum) and expansion may have lasted only up to the point of CMB emission (at this point the speed of light also became equal to c). The redshifts thus may be caused by lower scale $\left(\mathrm{U}_{-2}\right)$ particles in intergalactic medium absorbing photon energies.

Taking into account the scaled density of the observable universe (gas), evidently this is a discontinuity, a gravitational maximum between layers of, relatively, dense matter.

Since maximum speed in such discontinuities is Keplerian velocity (angular velocity of space) of the maximum, in this case equal to c, this is a black hole maximum (escape velocity $=\sqrt{2} \mathrm{c}$ ). 
Thus, light coming from large distances might be the light reflected off of the firewall, providing a window to the past of inner content. This explains the correlation of apparently spatially separated phenomena (galaxies) - these may not be images of different phenomena separated in space, but one separated in time (note that the decay, inverse decay of a graviton neutrino and photon can also explain the effect - neutrino and photon emitted from the same source may produce images of different luminosity if a neutrino decays to photon).

Note that, if one fixes the gravitational constant $G_{0}$ to

$$
G_{0}=1.257920328 * 10^{27} \frac{\mathrm{m}^{3}}{\mathrm{kgs}^{2}}
$$

one obtains this:

$$
\begin{aligned}
& \frac{G_{0} m}{k_{0} Q}=K^{-1} \mu_{0}^{-1}=\mu_{0}^{-1} \\
& c^{2}=4 \pi \frac{G_{0} m}{Q} K=4 \pi \frac{G_{0} m}{Q}
\end{aligned}
$$

where $\mu_{0}$ is the vacuum permeability (magnetic) constant and $\mathrm{K}=1 \mathrm{C} / \mathrm{m}$.

One can now obtain $\mathrm{k}$ and $\mathrm{Q}$ for the $\mathrm{U}_{1}$ scale (Solar System):

$$
\begin{gathered}
\frac{k_{1} Q_{1}{ }^{2}}{G_{1} m_{1}{ }^{2}}=\frac{k_{0} Q_{0}{ }^{2}}{G_{0} m_{0}{ }^{2}} \\
k_{1}=\frac{k_{0} Q_{0}{ }^{2}}{G_{0} m_{0}{ }^{2}} G_{1} \frac{c_{1}{ }^{4}}{16 \pi^{2} G_{1}{ }^{2}} K_{1}{ }^{-2}=\frac{k_{0} Q_{0}{ }^{2}}{G_{0} m_{0}{ }^{2}} \frac{c_{1}{ }^{4}}{16 \pi^{2} G_{1}}
\end{gathered}
$$

Using $\mathrm{G}_{1}=6.674 * 10^{-11} \mathrm{~m}^{3} / \mathrm{kgs}^{2}$ and previously obtained $\mathrm{c}_{1}=2.930445979$ $* 10^{6} \mathrm{~m} / \mathrm{s}$ :

$$
\begin{gathered}
k_{1}=3.95052951 * 10^{38} \frac{\mathrm{Nm}^{2}}{\mathrm{C}^{2}} \\
Q_{1}=10001.92779151 C \approx 1 * 10^{4} \mathrm{C}
\end{gathered}
$$

Ranges on $\mathrm{U}_{1}$ scale:

$$
\begin{gathered}
M_{\gamma_{1}}-M_{n 1}=\frac{\hbar_{1}}{c_{1}} \frac{1}{r} \ln \left(\frac{G_{1} m_{1}}{k_{1} Q_{1}}\right) \\
\hbar_{1}=\frac{h_{m 2}}{2 \pi}=7.95683841 * 10^{40} \mathrm{Js}
\end{gathered}
$$

Using $\mathrm{m}_{1}=1.02413 * 10^{26} \mathrm{~kg}$ and previously obtained $\mathrm{M}_{n_{1}}=1.663337576 *$ $10^{-26} \mathrm{~kg}, \mathrm{M}_{\gamma_{1}}=9.10938356 * 10^{-31} \mathrm{~kg}$, the distance where two forces become equal, $\mathrm{r}=1.0059686 * 10^{62} \mathrm{~m} \approx 1 * 10^{62} \mathrm{~m}$.

Note that, if one fixes $m_{1}$ to

$$
m_{1}=0.99026311 * 10^{26} \mathrm{~kg} \approx 1 * 10^{26} \mathrm{~kg}
$$


one obtains this:

$$
\frac{G_{1} m_{1}}{k_{1} Q_{1}}=K_{2} M_{p}=M_{p}
$$

where $\mathrm{M}_{p}=1.6726218977^{*} 10^{-27} \mathrm{~kg}$ is the mass of a standard proton.

Range of $\mathrm{U}_{1}$ electric force:

$$
\lambda_{\gamma_{1}}=\frac{\hbar_{1}}{c_{1}} \frac{1}{M_{\gamma_{1}}}=2.98069699 * 10^{64} \mathrm{~m} \approx 3 * 10^{64} \mathrm{~m}
$$

Range of $\mathrm{U}_{1}$ gravitational force:

$$
\lambda_{n 1}=\frac{\hbar_{1}}{c_{1}} \frac{1}{M_{n 1}}=\frac{\hbar_{1}}{5 * 10^{-20}}=1.63239937 * 10^{60} \mathrm{~m}
$$

Here, unit $\mathrm{m}$ (meter) is unscaled, for a properly scaled metric the ranges are equal to ranges on $\mathrm{U}_{0}$ scale.

\section{Relation of G variation to Sun's discontinu- ities}

Equalizing the strength of electric and gravitational force between two free particles (positron and electron), disregarding small mass of carrier particles:

$$
\begin{gathered}
k_{0} \frac{Q^{2}}{r^{2}}=G \frac{M^{2}}{r^{2}} \\
\frac{1}{4 \pi \varepsilon_{0}} Q^{2}=G M^{2}
\end{gathered}
$$

yields the following value for the gravitational constant $\mathrm{G}$ :

$$
\begin{aligned}
& G=\frac{k_{0} Q^{2}}{M^{2}}=2.78025476 * 10^{32} \frac{\mathrm{m}^{3}}{\mathrm{kgs}^{2}} \\
& \mathrm{k}_{0}=8.9875517873681764 * 10^{9} \mathrm{Nm}^{2} / \mathrm{C}^{2} \\
& \mathrm{Q}=1.60217733 * 10^{-19} \mathrm{C} \\
& \mathrm{M}=9.10938356 * 10^{-31} \mathrm{~kg}
\end{aligned}
$$

In $\mathrm{CR}$, gravitational constant $\mathrm{G}$ changes with scale. But it is also modified with neutralization of EM force, when $\mathrm{k}_{0}$ decreases, while $\mathrm{G}$ increases.

This enables the gravitational force to be, at least in some cases, a prevailing force in the atom, rather than EM force.

I have previously calculated $\mathrm{G}$ relative to a ${ }^{10} \mathrm{C}$ atom nucleus mass obtained through current Sun mass, the constant G using rest mass of ${ }^{10} \mathrm{C}$ nucleus is:

$$
G_{0}=v^{2} \frac{r}{M}=1.29864745 * 10^{27} \frac{\mathrm{m}^{3}}{\mathrm{kgs}^{2}}
$$




$$
\begin{array}{r}
\mathrm{v}=5.5550351679 * 10^{5} \mathrm{~m} / \mathrm{s} \\
\mathrm{r}=70^{*} 10^{-12} \mathrm{~m} \\
\mathrm{M}=1.663337576 * 10^{-26} \mathrm{~kg}
\end{array}
$$

where $\mathrm{m}, \mathrm{v}$ and $\mathrm{r}$ are components of the orbital angular momentum of the outermost electron.

Calculated $\mathrm{G}\left(\mathrm{G}_{0}\right)$ is now only 5 orders of magnitude smaller than $\mathrm{G}$ required for gravity to be equal in strength to EM force between an electron and a positron.

But instead of $\mathrm{G}$ increasing, one might assume that $\mathrm{k}_{0}$ decreases by 5 orders of magnitude, or more precisely by this amount:

$$
\Delta k=\frac{G}{G_{0}}=2.140884935 * 10^{5}
$$

Thus, the increase of $\mathrm{G}(\Delta \mathrm{G})$ of Earth's inner core maximum, after extraction, neutralization and collapse to current radii, is equal to $\Delta \mathrm{k}$.

I have previously calculated that this $\mathrm{G}$ has increased to $5.731534632 * 10^{-6}$ $\mathrm{m}^{3} / \mathrm{kgs}^{2}$, which is, relative to surface $\mathrm{G}\left(6.674 * 10^{-11} \mathrm{~m}^{3} / \mathrm{kgs}^{2}\right)$, an increase of:

$$
\Delta G=\frac{5.731534632 * 10^{-6}}{6.674 * 10^{-11}}=8.58785531 * 10^{4}
$$

which is also the ratio between imaginary mass M $\left(5.97 * 10^{24} \mathrm{~kg}\right)$ and real mass $\mathrm{m}\left(6.95 * 10^{19} \mathrm{~kg}\right)$ of Earth.

Gravitational constant G measured on the surface of the Earth (surface $\mathrm{G}$ ) is relative to standard scale $\left(\mathrm{U}_{0}\right)$, a proper $\mathrm{G}$ for $\mathrm{U}_{1}$ scale must be different.

But what was the initial G of Earth's inner core?

According to above hypothesis, it should have been:

$$
G_{i}=\frac{5.731534632 * 10^{-6}}{\Delta k}=2.677180141 * 10^{-11} \frac{\mathrm{m}^{3}}{\mathrm{kgs}^{2}}
$$

If Earth's core has been extracted from the Sun, as hypothesized, one can get it's original radius using this constant:

$$
\begin{array}{r}
r=\sqrt{\frac{G_{i} M}{g}}=440784499.323 m \approx 440785 \mathrm{~km} \\
\mathrm{M}=\operatorname{img} \text { mass of the Sun }=1.988500 * 10^{30} \mathrm{~kg} \\
\mathrm{~g}=\text { gravity of the maximum }=274 \mathrm{~m} / \mathrm{s}^{2}
\end{array}
$$

This agrees very well with the hypothesis of entanglement of discontinuities with inner planetary orbitals:

$$
\frac{r}{R} \approx \frac{r_{E}}{r_{M}} \approx \frac{2}{3}
$$




$$
\begin{array}{r}
\mathrm{R}=\text { Sun radius }=695700 \mathrm{~km} \\
\mathrm{r}_{E}=\text { Earth orbital }=149.6 * 10^{6} \mathrm{~km} \\
\mathrm{r}_{M}=\text { Mars orbital }=227.92 * 10^{6} \mathrm{~km}
\end{array}
$$

The discontinuity $(\mathrm{r} / \mathrm{R}=0.63)$ is evident through the profile of rotational velocities of the Sun:

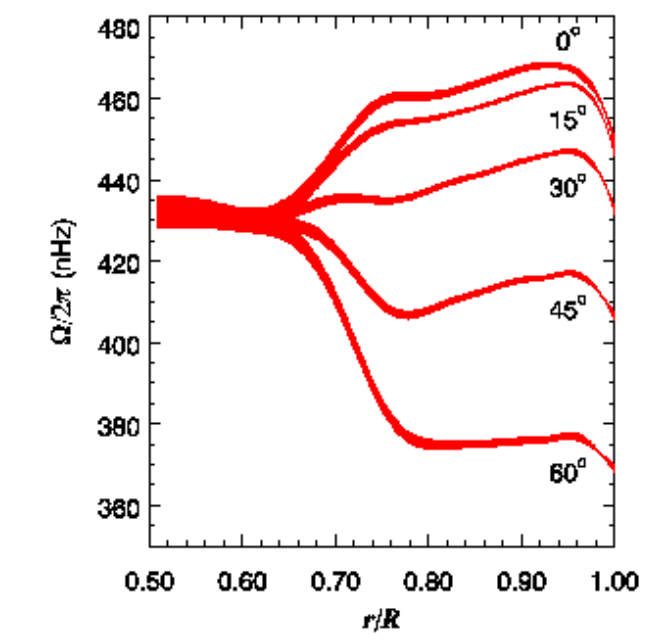

NSO/NSF

Figure 38: Sun rotation rates $^{80}$

Above this discontinuity is the tachocline (transition region between the radiative and convective layer of the Sun), a major source of the Sun's magnetic dipole, analogous to the region of charge above Earth's inner core.

The hypothesis of neurogenesis, assuming pending neurogenesis on Earth and completed neurogenesis on Mars and other terrestrial planets, explains why Earth is the only one with an active surface magnetic dipole. The connection of tachocline with $0.63 \mathrm{R}$ discontinuity would suggest:

1. it's position is not permanent and it moves between discontinuities, corresponding to the planet with ongoing neurogenesis,

2. possible multiple active discontinuities and associated tachoclines in the past, initially at maximum, or

3. current position is the place of birth of all planetary embryos (cores).

The 2nd hypothesis here is most plausible - the tachocline is active as long as the magnetic dipole of the corresponding planet is active (the two phenomena are synchronized).

However, if the tachocline is localized to $0.71 \mathrm{R}[81]$ and distance between the tachocline and the discontinuity is scaled from Earth (distance between the 
charge radius and gravitational maximum), the associated discontinuity is at:

$$
r=\frac{1206115}{1705704} 0.71 R=0.5 R \approx \frac{1}{\sqrt{2}} 0.71 R
$$

which would be a discontinuity associated with Venus.

In that case the tachocline is the location of a charge radius associated with a $0.5 \mathrm{R}$ gravitational maximum and, assuming equal g-factor, such charge radius should also be located at:

$$
r=\frac{1705704}{1206115} R=983868.265 k m \approx \sqrt{2} R
$$

In this case though, the g-factor of a neutron might be more appropriate, yielding $\mathrm{r}=1.111507303 * 10^{6} \mathrm{~km}$ (and a mirror at $444533.257 \mathrm{~km}=0.639 \mathrm{R}$ ).

Note that the $0.71 \mathrm{R}$ tachocline is $3 / 4$ of $0.94 \mathrm{R}$, which according to Fig. 38 seems to be another discontinuity or a fossilized initial Sun radius.

Such fossil is also visible at $0.75 \mathrm{R}$, which should be a discontinuity in $4 \mathrm{p} 6 \mathrm{n}$ state.

The $0.63 \mathrm{R}(2 / 3$ of $0.94 \mathrm{R})$ is also a fossil, as the current location associated with Earth is $0.66 \mathrm{R}$.

Note that $0.63 \mathrm{R}$ discontinuity is, similarly to $0.4 \mathrm{R}(2 / 5 \mathrm{R})$ discontinuity, weak (unstable) - it may not always be present in the rotational profile of the Sun.

The $0.63 \mathrm{R}$ has been revealed in seismic analysis (periodic, 1.3y signal), and possibly the $0.4 \mathrm{R}$ discontinuity too (noted as a low significance bump in rotation variability between $0.2 \mathrm{R}$ and $0.6 \mathrm{R})[82]$.

Sun's GM product has increased $0.06 \%$ due to kinetic energy relative to $\mathrm{CMB}$, so initial radius at $0.94 \mathrm{R}$ implies that surface radius changes proportionally:

$$
R=\frac{R_{0}}{\sqrt{1-\frac{v^{2}}{c_{1}^{2}}}}
$$

for previously obtained $\mathrm{c}_{1}=2.93 * 10^{6} \mathrm{~m} / \mathrm{s}$ and $\mathrm{v}=\mathrm{v}_{s}+\mathrm{v}_{p}=996 \mathrm{~km} / \mathrm{s}$, gives $\mathrm{R}_{0}=654271.142 \mathrm{~km}=0.94 \mathrm{R}$.

\section{Note 1:}

This is analogous to the decrease of Bohr radius due to relativistic mass of the electron. Bohr radius:

$$
a_{0}=\frac{\hbar}{m_{e} c \alpha}
$$


using relativistic mass:

$$
a_{r e l}=\frac{\hbar \sqrt{1-\frac{v_{e}^{2}}{c^{2}}}}{m_{e} c \alpha}
$$

It follows:

$$
\frac{a_{r e l}}{a_{0}}=\sqrt{1-\frac{v_{e}^{2}}{c^{2}}}
$$

Here, however, the radius of the atom is decreasing with the relativistic mass of electron, while the radius of the nucleus must increase with the relativistic mass of the gravitational maximum.

Note 2:

Although GM changes proportionally to R, differential rotation can shift discontinuities. Effectively, for the polar regions of the Sun, change is proportional with $\mathrm{R}^{2}$ :

$$
R^{2}=\frac{R_{0}^{2}}{\sqrt{1-\frac{v^{2}}{c_{1}^{2}}}}
$$

This gives $\mathrm{R}_{0}=0.97 \mathrm{R}$, and, according to Fig. 38 , it is indeed the correct value for polar regions. Note that the same discontinuity $(0.97 \mathrm{R})$ can be obtained is one assumes that gravity of Sun's surface maximum is invariant to changes in energy levels (Earth's maximum at inner core radius does suggest this to be true, which also implies equal species of the maximum for these two, if one is characterized by its gravity). In that case, with the loss of accumulated CMB kinetic energy, radius of the Sun decreases to $0.97 \mathrm{R}$.

If this discontinuity is correlated with the energy of real mass, and if decrease in radius has passed $0.97 \mathrm{R}$ in all regions apart from high polar region, the collapse of the surface maximum to $0.97 \mathrm{R}$ should be imminent.

Note also that with the end of 1st order cycle, collapse to $0.97 \mathrm{R}$ may be intermediary to larger collapse (up to core radius). Note that, taking the shift of $0.03 \mathrm{R}$ into account, $0.63 \mathrm{R}$ discontinuity becomes $0.66 \mathrm{R}$.

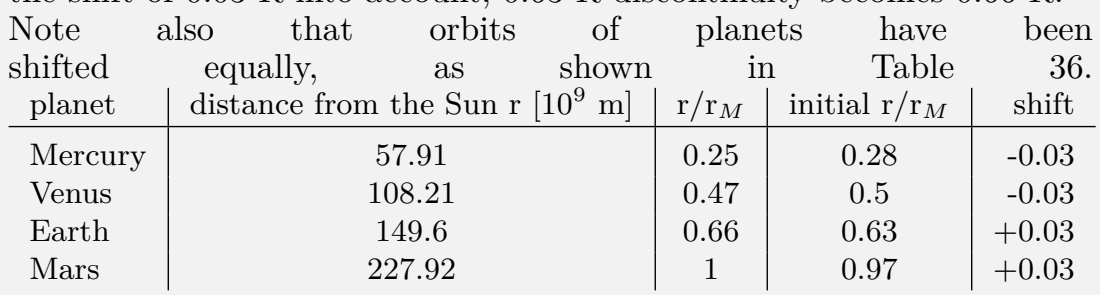

Table 36: Shifting of planetary orbits 
The Earth has thus moved from $0.63 \mathrm{r}_{M}$ to $0.66 \mathrm{r}_{M}$, while Venus moved equally but in opposite direction, from 0.5 to 0.47 . Mars moved from 0.97 to $1 \mathrm{r}_{M}$ and Mercury too moved accordingly.

Calculated initial radii, location of discontinuities and inner planets together with fossil evidence show the following:

- radii of gravitational maxima in the system change physically and proportionally to the change in momentum of the system,

- relativistic effects of time dilation and length contraction are a direct consequence of physical changes of space in the system (atom),

Kinetic energy changes the system on all scales, proportionally to the scale of a gravitational maximum. This constant of proportionality (or unit quanta) is the speed of light $\mathrm{c}$, which at the Sun scale $\left(\mathrm{U}_{1}\right)$ is equal to $\mathrm{c}_{1}=2.93 * 10^{6}$ $\mathrm{m} / \mathrm{s}$, while on the scale of the standard ${ }^{10} \mathrm{C}$ atom nucleus $\left(\mathrm{U}_{0}\right)$ is equal to $\mathrm{c}_{0}=$ $2.99792458 * 10^{8} \mathrm{~m} / \mathrm{s}$.

Note that all these constants are relative to a local universe characterized by the CMB (Constant Microwave Background) radiation.

CMB is thus not part of some special absolute space:

- if increase in kinetic energy increases the energy of the system and units of measurements (quanta of observational energy) then the initial state does not exist - what one considers the initial state of the system (and the current state of $\mathrm{CMB}$ ) is the result of kinetic energy of the space associated with CMB - the Solar System can thus be the actual standard carbon atom inflated due to this kinetic energy,

- but there is no reason for the carbon atom or any other particle to be something elementary, this goes to infinity.

The CMB space is thus only relatively special and it is so only due to limits in our senses (observational power) - we do not have the ability to detect radiation of the smaller scale than $U_{-1}$ photon, nor we have the ability to see how fast and relative to what the observable universe moves (we can only be pretty confident it rotates around something).

Our senses are effectively limited to the scale of the standard atom $\mathrm{U}_{0}$ (we are the composition of atoms of this scale) and adjacent vertical scales $\mathrm{U}_{1}$ and $\mathrm{U}_{-1}$. 


\section{Gyro-magnetic ratio and its correlation with Earth/Moon}

The gyro-magnetic ratio of a particle is the ratio of its magnetic moment to its angular momentum:

$$
\gamma=\frac{\mu}{L}
$$

With the assumption that mass and charge have equal momentum:

$$
\gamma=\frac{\mu}{L}=\frac{q}{2 m}
$$

where $\mathrm{q}, \mathrm{m}$ are charge and mass of the particle, respectively.

Measurements show that this is not valid for quantum particles such as electron. Thus, a dimensionless factor $\mathrm{g}_{e}$ (g-factor) was introduced:

$$
\gamma=\frac{q}{2 m} g_{e}
$$

The factor has been attributed to quantum effects which do not exist in classical (intuitive) reality - point particles with intrinsic magnetic moment (no rotation).

The notion of point particles having any properties is in itself problematic, let alone existence of different point particles with different properties. However, if such particles could exist, due to scale invariance, they would have to exist on bigger scales too. No such thing has ever been observed in reality - all magnetic fields are produced by moving charges of objects having a real radius.

Thus, intrinsic magnetic momentum is not intuitive, but intrinsic rotation of charge (producing the momentum) at finite radius greater than 0 is.

In CR there is also no intrinsic coupling of matter and gravity, and since charge field is a polarized gravitational field, the g-factor can be explained simply by a difference in distribution (or angular momenta) of gravitational mass and charge mass within the particle, preserving the intuitive concepts of reality.

Complete relativity not only allows speeds faster than light (photon mass is scale dependent) but implies such speeds must exist at some scale, thus the required superluminal rotation of charge (implied at certain radii) in particles such as an electron is not an issue either.

The absolute (invariant) speed limit is not a dimensionless constant and thus is counter-intuitive in scale invariant reality (relativity), but, in this case, the required speed would be valid even in the context of General Relativity (charge is at rest relative to rotating space) if it would incorporate scale invariant curvature of space.

Magnetic moment $\mu$ and angular momentum L:

$$
\begin{gathered}
\mu=I A=\frac{q v_{c}}{2 \pi r_{c}} \times \pi r_{c}{ }^{2} \\
L=m v_{m} \times r_{m}
\end{gathered}
$$


where $\mathrm{v}_{c}, \mathrm{r}_{c}$ are the charge orbital velocity and radius, respectively, and $\mathrm{v}_{m}, \mathrm{r}_{m}$ are the mass orbital velocity and radius, respectively.

The factor $\mathrm{g}_{e}$ is thus:

$$
g_{e}=\frac{v_{c} \times r_{c}}{v_{m} \times r_{m}}
$$

Being dimensionless, it should be scale invariant relative to particle flavor.

This means that the value of $\mathrm{g}_{e}$ for electron and positron is equal to $\mathrm{g}_{e}$ of Earth, as Earth is a large scale Dirac fermion equivalent (obviously not a point particle unless taken relatively), albeit in an environment where its fermionic nature is effectively annihilated.

Just like the electron, Earth consists of intrinsic charge and mass and accumulated mass due to neutralization.

The intrinsic energy is concentrated within the inner and outer core.

Assuming charge radius is in the outer core where gravity equals $\mathrm{g}_{c}=137$ $\mathrm{m} / \mathrm{s}^{2}$ and gravitational mass radius is the inner core gravitational maximum $\mathrm{g}_{m}$ $\left(274 \mathrm{~m} / \mathrm{s}^{2}\right)$, with equal rotation period (and angle between $\mathrm{v}$ and $\mathrm{r}$ vectors):

$$
g_{e}=\frac{v_{c} \times r_{c}}{v_{m} \times r_{m}}=\frac{r_{c}^{2}}{r_{m}^{2}}=\frac{g_{m}}{g_{c}}=2
$$

Note that it was assumed that mass is not a solid body with radius $r_{m}$ but, like the charge, a particle or a stream of particles forming a ring at $r_{m}$.

This is a valid assumption since this mass is not real mass, but vacuum energy (imaginary mass) which, in case of charged naked maxima, forms a ring rather than sphere surface.

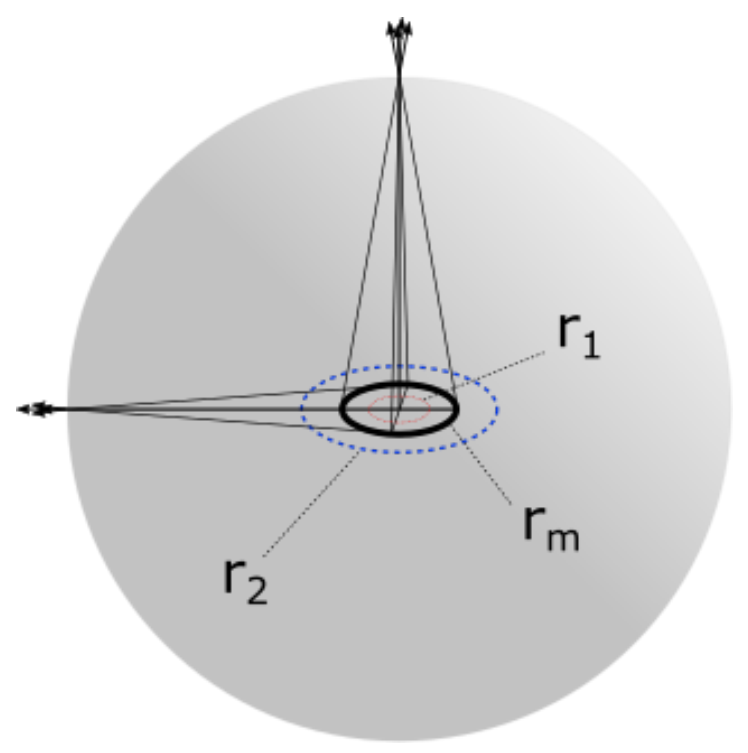

Figure 39: Mass and charge radius of charged bodies 
Since gravitational potential is not isotropic, gravitational acceleration at any point is a vector sum of accelerations induced by vacuum quanta forming the ring:

$$
g=\sum_{k=1}^{n} \overrightarrow{g_{k}}=\sum \frac{G_{0} M_{0}}{\overrightarrow{d r}^{2}}
$$

In case of equatorial and polar gravity vector components parallel to surface cancel out. Equatorial gravity is thus:

$$
g=\sum_{k=1}^{n} \frac{G_{0} M_{0}}{\left[R_{e}-r_{m} \cos \left(k \frac{2 \pi}{n}\right)\right]^{2}}
$$

where $\mathrm{R}_{e}$ is the equatorial radius.

Polar gravity:

$$
g=\sum_{k=1}^{n} \frac{G_{0} M_{0}}{R_{p}^{2}}=n \frac{G_{0} M_{0}}{R_{p}^{2}}
$$

where $\mathrm{R}_{p}$ is the polar radius.

Deriving $\mathrm{G}_{0} \mathrm{M}_{0}$ product with equatorial gravity fixed to $9.798 \mathrm{~m} / \mathrm{s}^{2}$ and calculating polar gravity, for $\mathrm{n}>=5$, gives $9.34 \mathrm{~m} / \mathrm{s}^{2}$.

This is smaller than measured, so the Earth must be a composite of 2 positrons (or positron equivalents), as hypothesized.

Note that I have previously hypothesized that the shape of a gravitational maximum with charge neutralization is transforming from a ring like to sphere surface form.

Here, it is assumed that ring form is preserved, for the sake of proving fossilization of initial conditions.

It is also assumed that gravity is provided by the gravitational maxima, rather than real mass [shielding the gravity of the maxima], however, effectively there is no difference for surface gravity (real mass imitates the maximum).

With 2 particles in the same state, energy splits into two levels:

In such state, two charges are deflected from the equator by this angle:

$$
\Delta \varphi=\sin ^{-1} \frac{1}{\sqrt{3}}=35.2643896827547^{\circ}
$$

Charges are thus separated by $2^{*} 35.2643896827547^{\circ}=70.5287793655094^{\circ}$ (two magnetic north poles on Jupiter are separated by this angle, confirming it's 2e configuration).

Confirmation of this configuration of Earth comes from the state of the Moon (Luna) and non-alignment of Earth's north and south magnetic poles. 

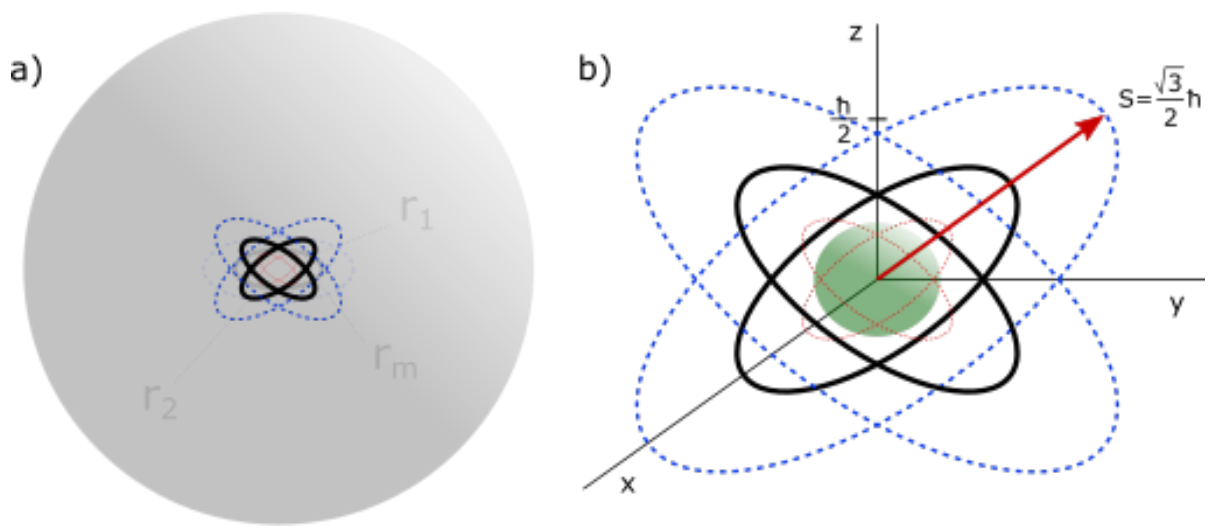

Figure 40: Mass and charge radius of two charged bodies sharing a single state

Total obliquity of Luna relative to Earth's equator is $23.44^{\circ}+5.14^{\circ}+6.68^{\circ}$ $=35.26^{\circ}$, equal to $\Delta \phi$.

The Moon orbits one of Earth's positrons and its obliquity shows that it is built around one of the collapsed gravitational maxima of this positron.

One can thus expect this positron to have smaller contribution to gravity and charge of Earth. Further splitting of energy levels due to carbon configuration can also be expected, so number of quanta should be 6 in one positron and 5 in the other ( 1 is in the Moon).

It appears that, in the collapse, $6.68^{\circ}$ of Luna's obliquity to Earth's equator has been exchanged for obliquity to Luna's own equator, this can be due to influence from another body, but, since the loss of one quantum causes asymmetry in charge distribution it is more likely that this is the exact amount by which the inner positron decreased its angle to Earth's equator.

Thus, one can expect the orbital plane of this positron to be aligned with the orbital plane of the Moon.

This can then be interpreted as redistribution of charges on the plane, rather than loss. The Moon is thus the reason why Earth still has a dipole magnetic field - with symmetric anti-aligned positron spins the magnetic dipole would be canceled.

Bigger moons and/or an increased number of moons (with distinct gravitational wells) of outer planets with stronger magnetic fields are thus no surprise and indicate core asymmetry if the spins are anti-aligned (note that a symmetric core does not indicate a planet has no moons, rather that it has the same number of them on each orbital plane).

But rather than the extraction of the Moon core from Earth, in the current, progressive evolution a reverse scenario is more plausible.

Even if the first positron was not fragmented from the beginning, massive extinctions that happened on Earth suggest the second one arrived quantum by quantum on a periodic basis.

There were 5 massive extinctions and there are 5 quanta of the positron in 
the core, 1 in the Moon.

As the mammal brain has 6 layers, with 6th layer sparsely populated, the theory of neurogenesis is strongly aligned with this hypothesis. Note that the sparse neuron cell population of the 6th layer now indicates an underdeveloped layer - the direct cause for this is the distance of the Moon.

Since this distance is variable it explains the variation in intelligence among individuals. A Moon in perigee at the point of formation of the 6th brain layer would increase general intelligence (at the time of formation of other layers would probably impact other skills).

This is not a big increase, but enough to create a difference and allow weak evolution of intelligence, as brain structure is a genetic factor.

Current increasing Moon distance and the fact that our brain size started decreasing 10-15k years ago support the hypothesis of such entanglement. As the Moon fuses with Earth, one can thus expect a strong evolution of the 6th layer in brains of species (including the brain of Earth itself). One must now ask whether the position of other planets and the Sun impact the development? Most likely, but not as much.

Interesting is the fact that one has 5 vital organs - these are thus likely entangled with other 5 quanta of the positron associated with the Moon, so variation in the state of these can be determined by organic variation between individuals. Strong disturbance could thus cause mutation in evolution.

Thus, one can not only expect our 6th brain layer to expand during the next strong evolution event, but also a new vital organ (a 6th sense) and mutation of a body into new species.

\section{Conclusion}

The aim of this paper was to provide good evidence for Complete Relativity, which, I am convinced, it has succeeded in. Indeed, the analysis reveals plenty of correlation and equivalence between small scale and large scale systems that cannot be easily dismissed as coincidence.

Strong correlation of Earth's mantle layers with major extinction events is a strong evidence for planetary neurogenesis. The existence of a discontinuity at $100 \mathrm{~km}$ depth even suggests that the formation of a mantle discontinuity precedes surface extinction. This shows that surface extinctions are programmed events, which, however, is not surprising for a neurogenesis of an evolving lifeform (one would expect for a brain layer to be at least roughly formed before neurons migrate to that layer).

A good theory is a theory with experimentally verifiable predictions. The $\mathrm{CR}$ and this analysis consist of many verifiable hypotheses and postulates. 
A lot of evidence already exists, but modern man may still be reluctant to accept it.

However, if the hypotheses on gravitational maxima, major extinction events and accelerated evolution in neurogenesis are correct, the time upon us will be collapsing the pillars of absolutism, one by one, in accelerating fashion.

\section{References}

[1] Complete Relativity: Nature of observables (2021), Amenoum https://amenoum.org/complete_relativity.html

[2] NASA Planetary Fact Sheet https://nssdc.gsfc.nasa.gov/planetary/factsheet/

[3] Images of planets (2013), OpenClipart-Vectors https://pixabay.com/users/openclipart-vectors-30363/

[4] Images of planets (2013), OpenClipart-Vectors https://pixabay.com/users/openclipart-vectors-30363/

[5] Six transiting planets and a chain of Laplace resonances in TOI-178 (2020), A. Leleu et al https://doi.org/10.1051/0004-6361/202039767

[6] CODATA recommended values (2018), NIST

https://physics.nist.gov/cuu/Constants/bibliography.html

[7] Six transiting planets and a chain of Laplace resonances in TOI-178 (2021), A. Leleu et al https://doi.org/10.1051/0004-6361/202039767

[8] Putovanje kroz vrijeme (2021), Amenoum https://amenoum.org/log/31_putovanje_kroz_vrijeme.html

[9] Rest mass of photon on the surface of matter (2020), M. Goray et al https://doi.org/10.1016/j.rinp.2019.102866

[10] An Electroweak model with electrons of opposite helicities carrying the same quantum numbers (1981), W. B. Yeung

[11] Long-term patterns of body mass and stature evolution within the hominin lineage (2017), M. Will et al https://doi.org/10.1098/rsos.171339

[12] Asymptotic g modes: Evidence for a rapid rotation of the solar core (2017), Fossat et al https://doi.org/10.1051/0004-6361/201730460 
[13] The Relationship of Sunspot Cycles to Gravitational Stresses on the Sun: Results of a Proof-of-Concept Simulation (2011), D. Jassby https://doi.org/0.1016/B978-0-12-385956-3.10014-2

[14] Internal rotation of Sun (2010), SOHO

https://ase.tufts.edu/cosmos/view_picture.asp?id=1368

[15] Velocity Curves for Spiral Galaxies (2015), C. R. Nave http://hyperphysics.phy-astr.gsu.edu/hbase/Astro/velcurv.html

[16] Discovery of variation in Solar coronal rotation with altitude (2001), H. O. Vats et al

[17] A Quantum-Like Description of the Planetary Systems (2007), F. Scardigli https://doi.org/10.1007/s10701-007-9151-7

[18] Self-Organizing Systems in Planetary Physics: Harmonic Resonances of Planet and Moon Orbits (2018), M. J. Aschwanden https://doi.org/10.1016/j.newast.2017.08.002

[19] Eccentricity of the geomagnetic dipole caused by lopsided inner core growth (2012), P. Olson et al https://doi.org/10.1038/NGEO1506

[20] Search for new phenomena in dijet events with the ATLAS detector at $\sqrt{s}$ $=13 \mathrm{TeV}$ (2019), D. Zhang https://cds.cern.ch/record/2683921/files/ATL-PHYS-SLIDE-2019426.pdf

[21] Signals of a $2 \mathrm{TeV}$ W' boson and a heavier Z' boson (2016), B. A. Dobrescu et al

https://doi.org/10.1007/JHEP05(2016)047

[22] Measurements of Newton's gravitational constant and the length of day (2015), J. D. Anderson et al https://doi.org/10.1209/0295-5075/110/10002

[23] Horizons On-Line Ephemeris System (2020), NASA Solar System Dynamics Group http://ssd.jpl.nasa.gov/

[24] Equatorial anisotropy in the inner part of Earth's inner core from autocorrelation of earthquake coda (2015), T. Wang et al https://doi.org/10.1038/ngeo2354

[25] A complex dynamo inferred from the hemispheric dichotomy of Jupiter's magnetic field (2018), K. M. Moore et al https://doi.org/10.1038/s41586-018-0468-5 
[26] Uranus fact sheet (2019), NASA/GSFC

https://nssdc.gsfc.nasa.gov/planetary/factsheet/uranusfact.html

[27] Neptune fact sheet (2019), NASA/GSFC

https://nssdc.gsfc.nasa.gov/planetary/factsheet/neptunefact.html

[28] 10Be: Half-life and AMS-standards (1987), H. J. Hofmann et al https://doi.org/10.1016/0168-583X(87)90198-4

[29] A new value for the half-life of 10Be by Heavy-Ion Elastic Recoil Detection and liquid scintillation counting (2010), G.Korschinek et al https://doi.org/10.1016/j.nimb.2009.09.020

[30] Rest Heart Rate and Life Expectancy (1997), H. J. Levine https://doi.org/10.1016/s0735-1097(97)00246-5

[31] Complete Relativity: Nature of observables (2021), Amenoum https://amenoum.org/complete_relativity.html\#outref1

[32] Athropogenic carbon and ocean pH, Nature 425, 365 (2003), K. Caldeira et al

[33] Ocean acidification and the Permo-Triassic mass extinction (2015), M. O. Clarkson et al https://doi.org/10.1126/science.aaa0193

[34] Essentials of geology, 4th edition, 256 (2013), S. Marshak

[35] Characteristic disruptions of an excitable carbon cycle (2019), D. H. Rothman https://doi.org/10.1073/pnas.1905164116

[36] Thresholds of catastrophe in the Earth system (2017), D. H. Rothman https://doi.org/10.1126/sciadv.1700906

[37] Do Red and Green Make Brown?: Perspectives on Plastid Acquisitions within Chromalveolates (2011), R. G. Dorrell et al https://doi.org/10.1128/EC.00326-10

[38] Future climate forcing potentially without precedent in the last 420 million years (2017), G. L. Foster et al https://doi.org/10.1038/ncomms14845

[39] Periodicity of extinctions in the geologic past (1983), D. M. Raup et al https://dx.doi.org/10.1073/pnas.81.3.801

[40] Periodic impact cratering and extinction events over the last 260 million years (2015), M. R. Rampino et al https://doi.org/10.1093/mnras/stv2088 
[41] No Evidence for a Large Atmospheric CO2 Spike Across the CretaceousPaleogene Boundary (2019), J. N. Milligan et al https://doi.org/10.1029/2018GL081215

[42] CO2 levels are rising (2018), Scripps Institution of Oceanography

[43] Understanding synchronicity (2020), Amenoum https://amenoum.org/log/19_understanding_synchronicity.html

[44] Amenoum (2020), Amenoum https://amenoum.org/authors/Amenoum.html

[45] 10Be $\beta$ - Decay Evaluated Data (2019), TUNL Nuclear Data Evaluation Project https://nucldata.tunl.duke.edu/nucldata/GroundStatedecays/10Be.shtml

[46] Where is the Earth's Radiogenic Helium? (1957), L. G. Carpenter et al https://www.nature.com/articles/179213a0

[47] Periodic impact cratering and extinction events over the last 260 million years (2015), M. R. Rampino et al https://doi.org/10.1093/mnras/stv2088

[48] Paleosol barometer indicates extreme fluctuations in atmospheric $\mathrm{CO} 2$ across the Cretaceous-Tertiary boundary (2002), L. Nordt et al https://doi.org/10.1130/0091-7613(2002)030\%3C0703:PBIEFI\%3E2.0.CO;2

[49] A new value for the half-life of 10Be by Heavy-Ion Elastic Recoil Detection and liquid scintillation counting (2010), G. Korschinek et al https://doi.org/10.1016/j.nimb.2009.09.020

[50] Dark matter's shadowy effect on Earth (2019), M. R. Rampino http://astronomy.com/magazine/2019/07/dark-matters-shadowy-effecton-earth

[51] Statistics of directional data (1972), K. V. Mardia

[52] Periodic impact cratering and extinction events over the last 260 million years (2015), M. R. Rampino et al https://doi.org/10.1093/mnras/stv2088

[53] Large igneous provinces and mass extinctions: An update (2014), D. Bond et al https://doi.org/10.1130/2014.2505\%2802\%29

[54] Permo-Carboniferous Volcanism in Europe and North Africa: a Superplume exhaust valve in The Center of Pangea (1998), M. Doblas et al https://doi.org/10.1016/S0899-5362\%2897\%2900138-3 
[55] Silurian Cycles: Linkages of Dynamic Stratigraphy with Atmospheric, Oceanic and Tectonic Changes (1998), L. Jeppsson

[56] Discovery of a major negative $\delta 13 \mathrm{C}$ spike in the Carnian (Late Triassic) linked to the eruption of Wrangellia flood basalts (2012), J. Dal Corso et al

https://doi.org/10.1130/g32473.1

[57] The Silurian Mulde Event and a scenario for secundo - secundo events (2007), L. Jeppsson et al https://doi.org/10.1017/s0263593300000377

[58] The Ireviken Event in the lower Silurian of Gotland, Sweden - relation to similar Palaeozoic and Proterozoic events (2003), A. Munnecke et al https://doi.org/10.1016/S0031-0182\%2803\%2900304-3

[59] The Kalkarindji Large Igneous Province, Australia: Petrogenesis of the Oldest and Most Compositionally Homogenous Province of the Phanerozoic (2018), B. D. Ware et al https://doi.org/10.1093/petrology\%2Fegy040

[60] Magmatic underplating beneath the Rajmahal Traps: Gravity signature and derived 3-D configuration (2004), A. P. Singh et al

[61] A Concise Geologic Time Scale (2016), F. M. Gradstein et al

[62] A Concise Geologic Time Scale (2016), F. M. Gradstein et al

[63] Mantle Discontinuities (2017), A. Deuss http://www.geo.uu.nl/ deuss/research/discontinuities/

[64] Imaging Mantle Heterogeneity with Upper Mantle Seismic Discontinuities (2015), N. Schmerr https://doi.org/10.1007/978-3-319-15627-9_3

[65] New theory of the Earth (2007), D. L. Anderson

[66] Mantle discontinuities (1991), C. R. Bina https://www.earth.northwestern.edu/ craig/publish/pdf/rg91.pdf

[67] Reflection of P'P' seismic waves from discontinuities in the mantle (1970), J. H. Whitcomb et al https://doi.org/10.1029/JB075i029p05713 
[68] Using the Baryonic Tully-Fisher Relation to Measure Ho (2020), J. Schombert et al https://doi.org/10.3847/1538-3881/ab9d88

[69] Measurement of Jupiter's asymmetric gravity field (2018), L. Iess et al https://doi.org/10.1038/nature25776

[70] Dynamics of Hippocampal Neurogenesis in Adult Humans (2013), Spalding et al https://dx.doi.org/10.1016/j.cell.2013.05.002

[71] Environmental Physiology of Animals (2009), P. Willmer

[72] A Sceptics View: 'Kleiber's Law' or the '3/4 Rule' is neither a Law nor a Rule but Rather an Empirical Approximation (2014), A. J. Hulbert https://doi.org/10.3390/systems2020186

[73] Tumor-derived TGF- $\beta$ inhibits mitochondrial respiration to suppress IFN$\gamma$ production by human CD4 + T cells (2019), S. Dimeloe et al https://dx.doi.org/10.1126/scisignal.aav3334

[74] Deadly 'two-faced' protein drives cancer growth, cripples T-cell avengers (2019), D. Ricks https://medicalxpress.com/news/2019-10-deadly-two-faced-proteincancer-growth.html

[75] UV surface brightness of galaxies from the local universe to z 5 (2014), E. J. Lerner et al https://doi.org/10.1051/0004-6361/201936373

[76] Evidence for anisotropy of cosmic acceleration (2019), J. Colin et al https://doi.org/10.1051/0004-6361/201936373

[77] Atomic Radii in Crystals (1964), J. C. Slater https://doi.org/10.1063\%2F1.1725697

[78] Covalent radii revisited (2008), B. Cordero et al https://doi.org/10.1039\%2Fb801115j

[79] Experimental tests of Coulomb's Law and the photon rest mass (2004), L-C. Tu et al https://doi.org/10.1088/0026-1394/41/5/S04

[80] The Pulse of the Solar Dynamo (2000), R. Howe et al http://soi.stanford.edu/press/GONG_MDI_03-00/

[81] An Introduction to the Solar Tachocline (2007), D. Gough https://doi.org/10.1017/CBO9780511536243.002

[82] Solar Interior Rotation and its Variation (2009), R. Howe https://doi.org/10.12942/lrsp-2009-1 